Georg-August-Universität Göttingen

Fakultät für Physik

Institut für Materialphysik

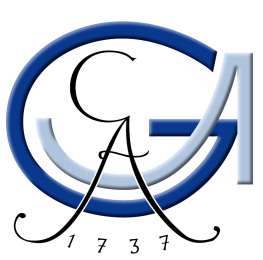

\title{
In situ studies on palladium/rutile titanium dioxide exposed to low pressure hydrogen gas environments
}

In situ Studien an Palladium/Rutil Titandioxid in Wasserstoffgasumgebungen geringen Druckes

\section{Dissertation}

zur Erlangung des mathematisch-naturwissenschaftlichen Doktorgrades

"Doctor rerum naturalium"

der Georg-August-Universität Göttingen

im Promotionsprogramm ProPhys

der Georg-August University School of Science (GAUSS)

vorgelegt von

Marian David Bongers

aus Kassel

Göttingen, 2018 
Betreuungsausschuss

apl. Prof. Dr. Astrid Pundt, Institut für Materialphysik,

Georg-August-Universität Göttingen

apl. Prof. Dr. Michael Seibt,

IV. Physikalisches Institut,

Georg-August-Universität Göttingen

Dr. Carsten Nowak,

Institut für Materialphysik,

Georg-August-Universität Göttingen

Mitglieder der Prüfungskommission

Referentin:

apl. Prof. Dr. Astrid Pundt,

Institut für Materialphysik,

Georg-August-Universität Göttingen

Korreferent:

apl. Prof. Dr. Michael Seibt,

IV. Physikalisches Institut,

Georg-August-Universität Göttingen

Weitere Mitglieder der Prüfungskommission:

Prof. Dr. Hans Hofsäss,

II. Physikalisches Institut,

Georg-August-Universität Göttingen

Prof. Dr. Reiner Kirchheim,

Institut für Materialphysik,

Georg-August-Universität Göttingen

apl. Prof. Dr. Vasily Moshnyaga,

I. Physikalisches Institut,

Georg-August-Universität Göttingen

PD Dr. Martin Wenderoth,

IV. Physikalisches Institut,

Georg-August-Universität Göttingen

Day of the oral examination: 05.02.2018 


\section{Contents}

$\begin{array}{lr}\text { 1. Introduction } & 1\end{array}$

2. Physical background of palladium and rutile titanium dioxide 5

2.1. Rutile titanium dioxide . . . . . . . . . . . . . . . . . 5

2.1.1. Symmetry and properties of $\mathrm{r}-\mathrm{TiO}_{2} \ldots \ldots . \ldots 7$

2.1.2. The $\mathrm{Ti} L$ edge of $\mathrm{r}-\mathrm{TiO}_{2}$ in electron energy loss spectroscopy (EELS) . . . . . . . . . . . . . . . . 11

2.1.2.1. Electron beam damage . . . . . . . . . . . . . 15

2.1.3. Intrinsic defects $\mathrm{r}-\mathrm{TiO}_{2} \ldots \ldots \ldots \ldots . \ldots . \ldots . \ldots$

2.1.4. Hydrogen interactions with $\mathrm{r}^{-\mathrm{TiO}_{2}} \ldots \ldots . \ldots 26$

2.1.4.1. Hydrogen diffusion in $\mathrm{r}^{-\mathrm{TiO}_{2}}$. . . . . . . . . . . . . 29

2.2. Palladium $(\mathrm{Pd}) \ldots \ldots \ldots \ldots$. . . . . . . . . . . . . . . 33

2.3. Palladium/rutile titanium dioxide $\left(\mathrm{Pd} / \mathrm{r}-\mathrm{TiO}_{2}\right) \ldots \ldots . \ldots . . . . .35$

2.3.1. Growth and expected lattice misfit of $\mathrm{Pd}$ on $\mathrm{r}-\mathrm{TiO}_{2} \ldots \ldots$. . . . 36

2.3.1.1. Thermally induced mechanical stress . . . . . . . . 38

2.3.2. Electrical properties of metal/semiconductor contacts (MSC) . . 40

2.3.2.1. Influence of hydrogen on metal $/ \mathrm{r}-\mathrm{TiO}_{2}$ contacts . . . . 42

3. Experimental methods and advanced EELS methodology 49

3.1. Magnetron sputtering . . . . . . . . . . . . . . . . 50

3.1.1. Pretreatment of the $\mathrm{r}-\mathrm{TiO}_{2}$ crystals . . . . . . . . . . 50

3.1.2. Oxygen treatment and Palladium film deposition . . . . . . . 51

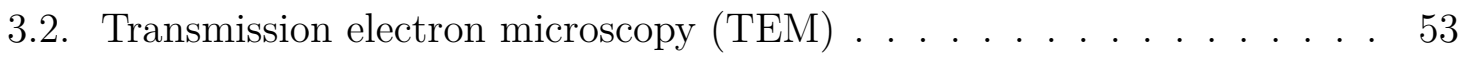

3.2.1. Preparation of TEM lamellae . . . . . . . . . . . . . 53

3.2.2. In situ EELS experimental procedure on $\mathrm{Pd} / \mathrm{r}-\mathrm{TiO}_{2}$. . . . . . . 54

3.2.3. In situ hydrogen loading experiments on $\mathrm{Pd}$ films . . . . . . . . 55

3.3. Electron energy loss spectroscopy (EELS) . . . . . . . . . . . . . 57

3.3.1. Preceding EELS parameter refinement . . . . . . . . . . 58

3.3.1.1. Monochromized zero loss peak (ZLP) description and energy resolution improvement . . . . . . . . . 58

3.3.1.2. Stabilization of energy stability, energy drift and spatial drift . . . . . . . . . . . . . . . . . 60

3.3.1.3. Pre-edge background and spectrometer dispersion impact .................... 66

3.3.1.4. Optimization of the interface alignment . . . . . . 67 
3.3.1.5. Influences of carbon (C) contamination and cold trap . 67

3.3.2. Developed advanced EELS methodology . . . . . . . . . . . 70

3.3.2.1. Spectra acquisition . . . . . . . . . . . . 70

3.3.2.2. Evaluation procedure and automatization . . . . . . 71

3.3.3. Evaluation of physical parameters . . . . . . . . . . . 75

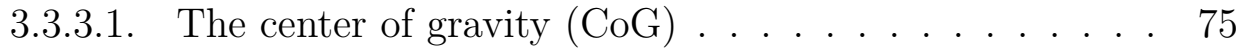

3.4. Positron annihilation spectroscopy (PAS) . . . . . . . . . . . . 77

3.5. X-ray investigation methods . . . . . . . . . . . . . . . . . . 79

3.5.1. X-ray diffraction (XRD) . . . . . . . . . . . . . . 79

3.5.1.1. XR diffractogram of a $\operatorname{Pd}(111)$ single crystal . . . . . . 81

3.5.1.2. Normal strain in thin films . . . . . . . . . . . . . . 82

3.5.1.3. Stress free lattice parameter of thin Pd film . . . . . . 82

3.5.1.4. Pole figures and $\phi$ scans . . . . . . . . . . . . 83

3.5.2. X-ray reflectometry $(\mathrm{XRR}) \ldots \ldots \ldots$. . . . . . . 84

3.5.3. Diffractometer details . . . . . . . . . . . . . . . . 85

3.6. X-ray photoelectron spectroscopy (XPS) . . . . . . . . . . . . . 87

3.7. Atomic force microscopy $(\mathrm{AFM}) \ldots \ldots$. . . . . . . . . . . 87

3.8. Density functional theory (DFT) on the hydrogen-r- $-\mathrm{TiO}_{2}$ system . . . . 88

4. Results 91

4.1. $\mathrm{Pd} / \mathrm{r}-\mathrm{TiO}_{2}$ sample preparation and characterization of the initial state . 91

4.1.1. The $\mathrm{r}-\mathrm{TiO}_{2}$ crystal surface . . . . . . . . . . . . . . 91

4.1.2. Pd films on pretreated $\mathrm{r}-\mathrm{TiO}_{2}$ crystals . . . . . . . . . . . 93

4.1.2.1. Thick Pd films prepared at $1023 \mathrm{~K} \ldots$. . . . . . . 94

4.2. $I$ - $V$ characterization of hydrogen loaded $\mathrm{Pd} / \mathrm{r}-\mathrm{TiO}_{2} \ldots \ldots \ldots$

4.3. Local EELS on hydrogen loaded $\mathrm{Pd} / \mathrm{r}-\mathrm{TiO}_{2} \ldots \ldots$. . . . . . . . . 101

4.3.1. Hydrogen induced center of gravity (CoG) shifts . . . . . . . . 106

4.4. DFT calculation of oxygen and hydrogen related defects in $\mathrm{r}^{-\mathrm{TiO}_{2}}$. . . 107

4.4.1. Thermodynamic stability and $\mathrm{Pd} / \mathrm{r}-\mathrm{TiO}_{2}$ interface investigation 108

4.4.2. Atomic structure and local symmetry of $\mathrm{H}_{\mathrm{O}}$ in $\mathrm{r}-\mathrm{TiO}_{2}$. . . . . 113

4.4.3. $\mathrm{H}_{\mathrm{O}}$ induced changes in the $\mathrm{r}-\mathrm{TiO}_{2}$ density of states (DoS) . . . . 114

4.4.4. Center of Gravity (CoG) from the DoS calculations . . . . . . . 118

4.4.4.1. A different approach . . . . . . . . . . . . . . 121

4.5. Defect concentrations in the $\mathrm{Pd} / \mathrm{r}-\mathrm{TiO}_{2}$ interface vicinity . . . . . . . . 122

4.5.1. A different approach . . . . . . . . . . . . . . . . . . . 124

4.6. Microstructural interface changes in $\mathrm{r}-\mathrm{TiO}_{2}$ due to hydrogen loading . . 126

4.7. PAS measurements on hydrogen loaded $\mathrm{Pd} / \mathrm{r}-\mathrm{TiO}_{2}$. . . . . . . . . 128

4.8. ETEM investigations on hydrogen related changes in $\mathrm{Pd}$. . . . . . . 131

5. Discussion $\quad \mathbf{1 3 5}$

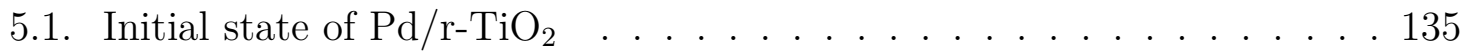

5.2. $\mathrm{Pd} / \mathrm{r}-\mathrm{TiO}_{2}$ equilibration with the hydrogen gas environment in the ETEM139 
5.3. Hydrogen induced changes within 'bulk' $\mathrm{r}^{-\mathrm{TiO}_{2}}$. . . . . . . . . . . . 140

5.4. Hydrogen induced changes within the $\mathrm{Pd}$ film . . . . . . . . . . . . . . 141

5.5. Hydrogen induced changes at the $\mathrm{Pd} / \mathrm{r}-\mathrm{TiO}_{2}$ interface and in its vicinity 143

5.6. Consequences for the electronic behavior: mobile species . . . . . . . 147

6. Summary

A. Appendix

155

A.1. Addition to theory . . . . . . . . . . . . . . . . . . 155

A.2. Thin Pd film prepared at room temperature (RT) . . . . . . . . . 156

A.3. X-ray investigations . . . . . . . . . . . . . . . . 160

A.3.1. Pole figure measurements and $\phi$ scans . . . . . . . . . . . 160

A.3.2. X-ray reflectometry . . . . . . . . . . . . . . . 161

A.4. Constraints for the lateral stress calculation . . . . . . . . . . . . 161

A.5. Alignment of the TEM and EELS acquisition parameters . . . . . . . 162

A.6. Local EELS on hydrogen loaded Pd/rutile . . . . . . . . . . . . . . . . 164

A.7. Formulas . . . . . . . . . . . . . . . . . . . . . . . 164

$\begin{array}{ll}\text { Bibliography } & 167\end{array}$

$\begin{array}{ll}\text { Acknowledgment } & 183\end{array}$ 



\section{Introduction}

During the 20th century, the comprehension and control of surface and interface properties became important. This is especially true for nano-sized materials where the interface properties play a dominant role in the overall material properties. Bulk and surface, though, are strongly correlated and have to be treated as one entity. Correspondingly, the near surface/interface regions (subsurface) are important as well. Thus, a fundamental understanding of the interface and its vicinity is necessary to tune the properties of the whole material.

This very general statement is present in many aspects of modern material physics and can be further generalized by taking all defects of the material into account. Thus, the investigation of this 'defective matter' plays a key role in the way to design and tune the properties of a material to a specific purpose.

A challenging task of today is the advent of sustainability as a focus of daily life. Sustainable energy production is one important example. A fully sustainable hydrogen cycle is a promising solution for a sustainable energy supply [1], using sunlight to produce chemical energy that can be subsequently be released in a fuel cell. For the production of solar-hydrogen, titanium dioxide $\left(\mathrm{TiO}_{2}\right)$ is a promising candidate as a photo-anode material [1].

In today's industrial applications, $\mathrm{TiO}_{2}$ is used mainly as a pigment $[2,3]$ because of its high refractive index. The pigments are used in paints, toothpastes, plastics, textiles and sunscreen creams [3]. However, $\mathrm{TiO}_{2}$ has a high potential for future applications in industry [3-6]. Some examples are photocatalytic water purification, hydrogen generation by water splitting, functional coating for building materials [3], highpermittivity dielectric or rectifier [4], dye-sensitized solar cells, self-cleaning paints, and antibacterial surfaces $[5,6]$.

All these possible applications demonstrate the high potential of $\mathrm{TiO}_{2}$ and clarify the importance of a fundamental understanding of $\mathrm{TiO}_{2}$ and related systems, such as metal $/ \mathrm{TiO}_{2}$ systems.

In 1972, Fujishima and Honda [7] presented the possibility of splitting water without applying an external potential, by the presence of light with a suitable wavelength. The work was done by irradiating a $\mathrm{TiO}_{2}$ anode in an electrochemical cell with a platinum cathode. This experiment drew a lot of attention to $\mathrm{TiO}_{2}$ as a functional material for photocatalysis, and to energy science in general.

Nowotny et al. [1] summarized recent prospects and challenges in the conversion of solar energy stored in hydrogen. They expect a high potential of solar-hydrogen. The advantages are [1] that hydrogen exhibits the highest energy capacity per unit mass 
of any known fuels, that the combustion is free of pollution, and that the production of solar-hydrogen can be done in regions that are politically stable. A crucial step in the hydrogen energy cycle is the conversion of solar (light) to chemical energy (hydrogen). For this purpose, functional materials (catalysts) are needed that convert the energy efficiently and that are stable for long times in the energy conversion. Here, reduced titanium dioxide $\left(\mathrm{TiO}_{2-x}\right)$ is the most promising candidate [1,5]. First of all, $\mathrm{TiO}_{2-x}$ is cheap relative to other conversion materials. Second, it is resistant against corrosion and photo-corrosion processes in the aqueous environment $[1,3]$ of a photoelectrochemical cell. While these are very important properties, the energy conversion efficiency of $\mathrm{TiO}_{2}$ is still low at $1 \%$ [1]. A suitable target for commercialization is $10 \%$ [1]. To increase the energy conversion efficiency, Nowotny et al. [1] see the need to investigate and understand the corresponding material properties including, the defect chemistry and the related electronic structure, the semiconducting properties, the composition, the nonstoichiometry, and the microstructure. Of particular interest are the surface active centers of $\mathrm{TiO}_{2-x}$ as well as the Schottky barrier that needs to be established on the anode's surface for charge separation [1]. For example, both can be modified by the presence of impurity atoms. This proves the point of how important a knowledge of interfaces is, especially with regards to their interaction with the environment.

Aside from the production of solar-hydrogen, hydrogen plays a central role in modern industry. Hydrogen is used [8] in the production of fossil fuels, ammonia, methanol, hydrochloric acid, and as a hydrogenating and reducing agent of catalysts to improve their activity [9], to name some examples. In these processes, the hydrogen concentration is an important issue and, consequently, gas sensors play an important role. Gas sensing on metal/semiconductor contacts (MSC) and their changes in a hydrogen environment are possible applications for gas sensing. Here, the current (density) at a given voltage, which is, in the following, abbreviated with its 'conductivity', of the MSC depends strongly on the applied hydrogen (partial) pressure. Palladium (Pd) and platinum $(\mathrm{Pt})$ on $\mathrm{TiO}_{2}$ have been studied as model systems in this field [10-13]. A general understanding of the involved physical interaction and processes has already been established [10-13]: Hydrogen is dissociated on $\mathrm{Pd}$ or Pt, and diffuses as atomic species to the interface of the MSC where it spills over into the interface and the semiconductor. There it enhances the conductivity of the overall MSC which results in the sensing effect. It is suggested that the enhanced conductivity mainly results from an interface and near-interface effect in the $\mathrm{TiO}_{2}[10,11]$. However, the microscopic details of the hydrogen interaction are still unclear $[12,13]$. The hydrogen sensitivity was found to be highest for $\mathrm{Pd} / \mathrm{TiO}_{2}$ when compared to $\mathrm{Pt}$ and gold [10]. This makes the $\mathrm{Pd} / \mathrm{TiO}_{2}$ system the ideal candidate for the investigation of hydrogen related changes close to the interface.

Comparing catalysis and gas sensing, the underlying processes are closely related. While in catalysis, it is important how the material (catalyst) interacts with the reactants, in gas sensing it is important how the reactants interacts with the material 
(catalyst). It is obvious that both processes are connected and are each one side of the same coin.

The aim of this work is to give additional insights into the interaction of hydrogen with the $\mathrm{Pd} /$ rutile $\mathrm{TiO}_{2}$ system and thereby build up a fundamental understanding of the underlying process(es). For that purpose, hydrogen induced changes are investigated with in situ techniques in the $\mathrm{Pd}$, in the bulk of rutile $\mathrm{TiO}_{2}$ and in the interface vicinity.

The word 'interface' describes the plane between the atomic row of the Pd that is neighbouring the first atomic row of the $\mathrm{TiO}_{2}$. The interface is indicated with a black line in fig. 1.1. It is therefore the bonding plane between the $\mathrm{Pd}$ and $\mathrm{TiO}_{2}$ lattice. The interface 'vicinity' describes a region from the interface into the $\mathrm{TiO}_{2}$ with an extension of approximately $20 \mathrm{~nm}$, cf. fig. 1.1. For simplicity rutile $\mathrm{TiO}_{2}$ is referred to as $\mathrm{r}-\mathrm{TiO}_{2}$ in this thesis.

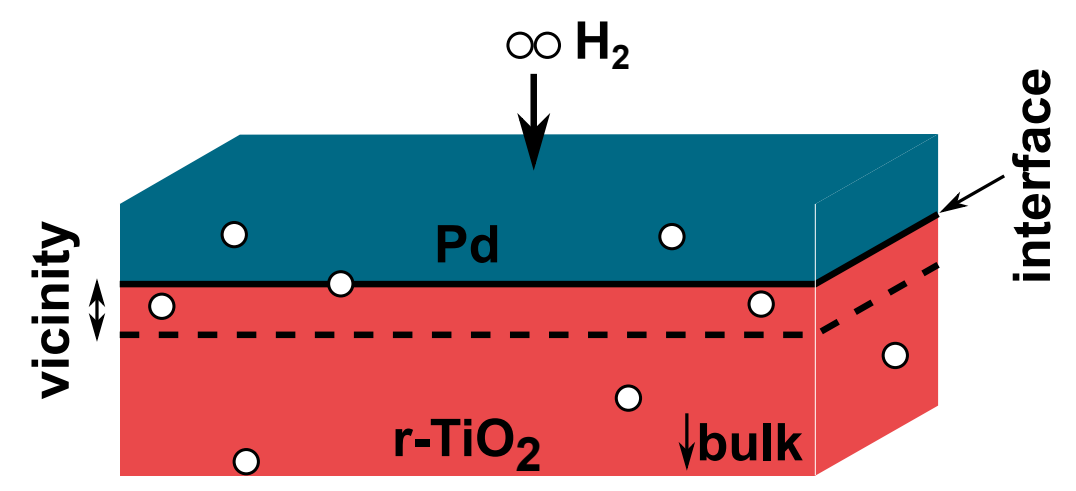

Figure 1.1.: Sketch of the $\mathrm{Pd} / \mathrm{r}-\mathrm{TiO}_{2}$ system in a hydrogen gas atmosphere. Indicated with a black line is the $\mathrm{Pd} / \mathrm{r}-\mathrm{TiO}_{2}$ interface. The black rectangle indicates the EELS investigated area in the interface vicinity. Coloring after CPK coloring of 'Jmol' [14].

Hydrogen induced changes in Pd films are investigated with positron annihilation spectroscopy (PAS) to reveal open volume defects created in the hydrogen environment. Complementary, high resolution scanning transmission electron microscopy (HR-STEM) is applied in situ in an environmental transmission electron microscope (ETEM). Here, the appearance of dislocations in the Pd film is studied as a function of the hydrogen gas pressure.

Hydrogen induced changes in bulk $\mathrm{r}-\mathrm{TiO}_{2}$, cf. fig. 1.1, are investigated in different hydrogen environments. Here, PAS allows the investigation of changes in the titanium vacancy concentration. In addition, electron energy loss spectroscopy (EELS) examines hydrogen related changes in the $\mathrm{Ti} L$ edge.

The interface vicinity in $\mathrm{r}-\mathrm{TiO}_{2}$, cf. fig. 1.1, is investigated with EELS in the ETEM. For this task, a new local in situ approach is developed: In the ETEM, the local chemistry of $\mathrm{TiO}_{2}$ in the vicinity of the $\mathrm{Pd} / \mathrm{TiO}_{2}$ interface region is studied with EELS at different hydrogen pressures. 
This procedure has high requirements for the EELS measurement process as well as for the prepared TEM lamella. A sufficient energy stability and a high energy resolution are required to investigate small energetic changes in EEL spectra in the interface near region. The $\mathrm{Pd} / \mathrm{r}-\mathrm{TiO}_{2}$ sample should exhibit a sharp interface with a high crystallinity of $\mathrm{Pd}$ and $\mathrm{r}-\mathrm{TiO}_{2}$ to precisely measure an interface distance dependency and to minimize the influence of microstructure on the obtained results.

Density functional theory (DFT) is applied on $\mathrm{r}-\mathrm{TiO}_{2}$ to investigate the influence of different defects to the density of states (DoS). The related DFT calculations have been performed by M. Sotoudeh and P. Blöchl. These calculations can help to interpret the experimentally observed hydrogen induced changes in the $\mathrm{Pd} / \mathrm{TiO}_{2}$ system.

This thesis is divided into six chapters. Most chapters have a summary with the most important information with respect to the focus of this work. The physical background chapter 2 establishes a fundamental knowledge about $\mathrm{TiO}_{2}$, with focus on the rutile modification, as well as the application of EELS on $\mathrm{r}-\mathrm{TiO}_{2}$. Furthermore, the intrinsic defect structure of $\mathrm{r}-\mathrm{TiO}_{2}$ and its known interaction with hydrogen is summarized. Important physical parameters of $\mathrm{Pd}$ and its interaction with hydrogen are presented. The chapter closes with an overview of the $\mathrm{Pd} / \mathrm{r}-\mathrm{TiO}_{2}$ system including mechanical and electrical properties as well as its known interaction with hydrogen. The experimental methods used are presented in chapter 3 . The optimized preparation of the $\mathrm{Pd} / \mathrm{r}-\mathrm{TiO}_{2}$ system as well as the techniques used for its characterization are presented here. A detailed view is given on the developed EELS methodology and the local in situ hydrogen loading experiments performed in the ETEM. Additionally, a background for the density functional theory calculations is introduced. The obtained results are presented in chapter 4 . This chapter begins with the characterization of the produced $\mathrm{Pd} / \mathrm{r}-\mathrm{TiO}_{2}$ system and its optimization. Here, the main results are presented: The investigation of the interface vicinity via in situ EELS investigations is shown. Positron annihilation spectroscopy studies and high resolution investigations in the ETEM are presented as well. Furthermore, DFT calculations are presented. The discussion in chapter 5 links the established knowledge with the obtained results. Here, a final picture is derived for the hydrogen induced microstructural changes that were observed in the $\mathrm{Pd} / \mathrm{r}-\mathrm{TiO}_{2}$ system in hydrogen atmosphere. This work is summarized in chapter 6 and the aims defined in the introduction are discussed on the basis of the performed experiments. Additionally, an outlook is given for possible future studies. 


\section{Physical background of palladium and rutile titanium dioxide}

In this chapter, fundamental backgrounds are established. The palladium/rutile titanium dioxide material $\left(\mathrm{TiO}_{2}\right)$ system is introduced as well as its interaction with hydrogen. The basics of electron energy loss spectroscopy and the corresponding titanium $L$ edge is given and its application on rutile $\mathrm{TiO}_{2}\left(\mathrm{r}-\mathrm{TiO}_{2}\right)$.

Chap. 2.1 presents rutile titanium dioxide and its properties and structure. A connection between the symmetry and the corresponding energy states is established in chap. 2.1.1. The basics about the symmetry of rutile and the symmetry of an octahedron are not discussed here and can be found elsewhere, for example in [15] and [16, chap. 3.20], respectively. The energetic states in rutile and their connection to the Ti $L$ edge is discussed in the context of electron energy loss spectroscopy (EELS) in chap. 2.1.2. The intrinsic defect chemistry of $\mathrm{r}-\mathrm{TiO}_{2}$ and its dependency on the oxygen environment are summarized in chap. 2.1.3. The interaction of $\mathrm{r}-\mathrm{TiO}_{2}$ and its defects with hydrogen is discussed in chap. 2.1.4.

Palladium $(\mathrm{Pd})$ and its important properties are discussed in chap. 2.2.

This work focuses on the $\mathrm{Pd} / \mathrm{r}-\mathrm{TiO}_{2}$ material system and not on the individual materials. The physical properties of this system are presented in chap. 2.3. Details are given with respect to the metal/semiconductor properties as well as to the interaction with hydrogen.

\subsection{Rutile titanium dioxide}

Titanium $(\mathrm{Ti})$ and oxygen $(\mathrm{O})$ can react to form a variety of different titanium oxide $\left(\mathrm{Ti}_{\mathrm{y}} \mathrm{O}_{\mathrm{x}}\right)$ phases in thermal equilibrium [17]. The resulting phase is dependent on the available amount of $\mathrm{Ti}$ and $\mathrm{O}$ species, as shown in the Ti-O phase diagrams in fig. 2.1. The stoichiometry of $\mathrm{Ti}_{\mathrm{y}} \mathrm{O}_{\mathrm{x}}$ starts with pure $\mathrm{Ti}$ and continues with $\mathrm{Ti}_{3} \mathrm{O}_{2}$, $\mathrm{TiO}, \mathrm{Ti}_{2} \mathrm{O}_{3}, \mathrm{Ti}_{3} \mathrm{O}_{5}$, a region of the so-called Magnéli phases [20] $\mathrm{Ti}_{n} \mathrm{O}_{2 n-1}$, where $n$ is typically between 4 and 10, but higher $n$ values were also reported [17], and ends with $\mathrm{TiO}_{2}$. The $\mathrm{TiO}_{2}$ phase is stable between approximately $\mathrm{TiO}_{1.99}$ and $\mathrm{TiO}_{2}$ [21].

The Ti atoms appear in three formal oxidation states for the oxide phases between $\mathrm{TiO}$ and $\mathrm{TiO}_{2}$ [22], cf. fig. 2.1: $2+$ for $\mathrm{TiO}, 3+$ for $\mathrm{Ti}_{2} \mathrm{O}_{3}, 3+$ and $4+$ in different ratios for the Magnéli phases, and $4+$ in $\mathrm{TiO}_{2}$. In all of these oxides, oxygen atoms 


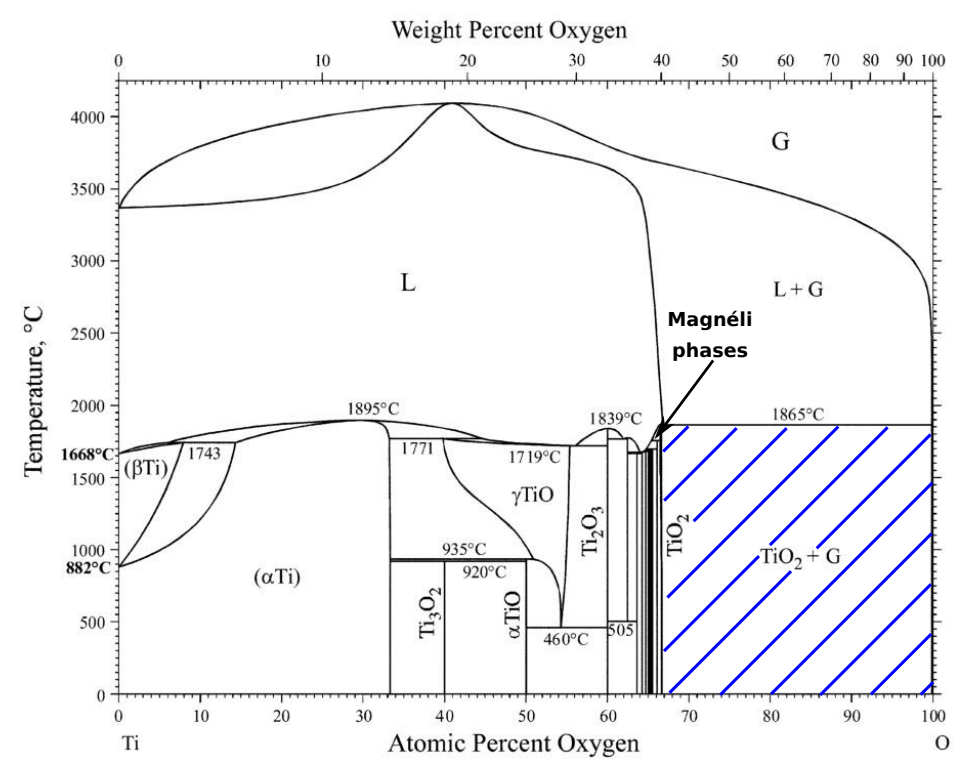

(a) Adapted with permission from Okamoto [18]. Copyright 2011 Springer Nature.

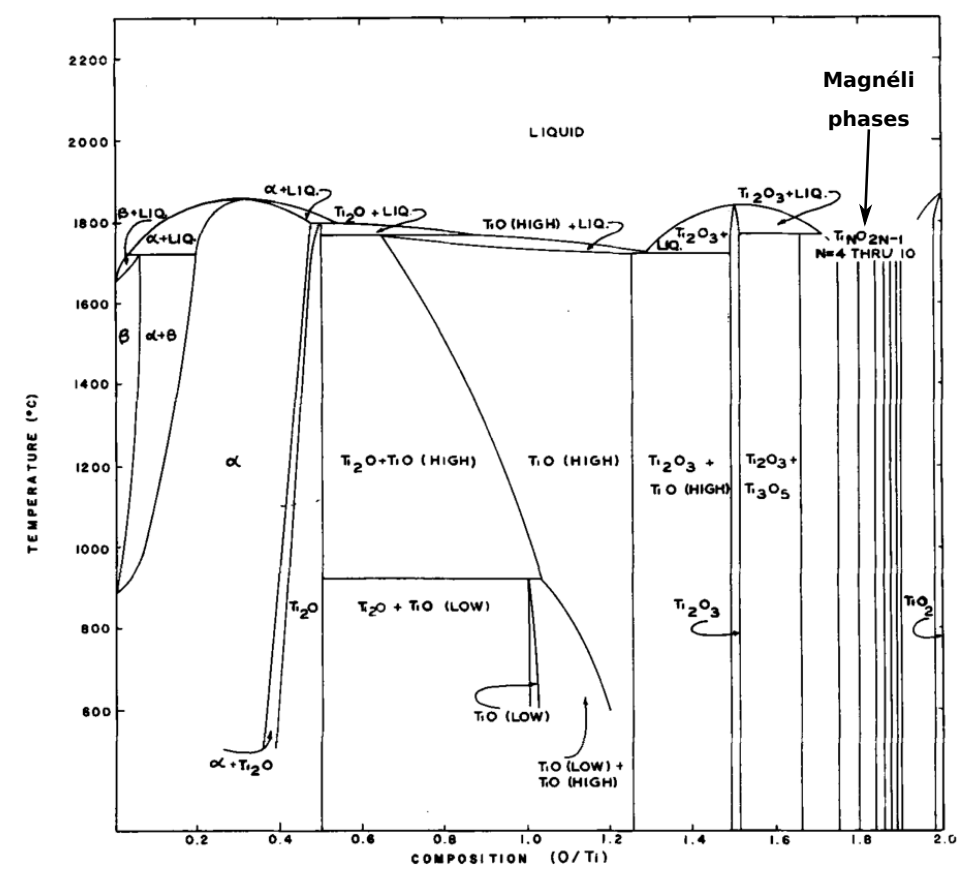

(b) Adapted with permission from Wahlbeck [19]. Copyright 2006 John Wiley and Sons.

Figure 2.1.: Titanium (Ti) - oxygen $(\mathrm{O})$ phase diagram. (a) classic view and (b) $\mathrm{O} / \mathrm{Ti}$ ratio view. Titanium dioxide $\left(\mathrm{TiO}_{2}\right)$ forms at approximately $67 \mathrm{at} \%$ of $\mathrm{O}$ or at an $\mathrm{O} / \mathrm{Ti}$ ratio at or above $2: 1$. 
arrange into octahedra around the Ti atoms. The octahedra of selected titanium oxide phases are shown in fig. 2.2.

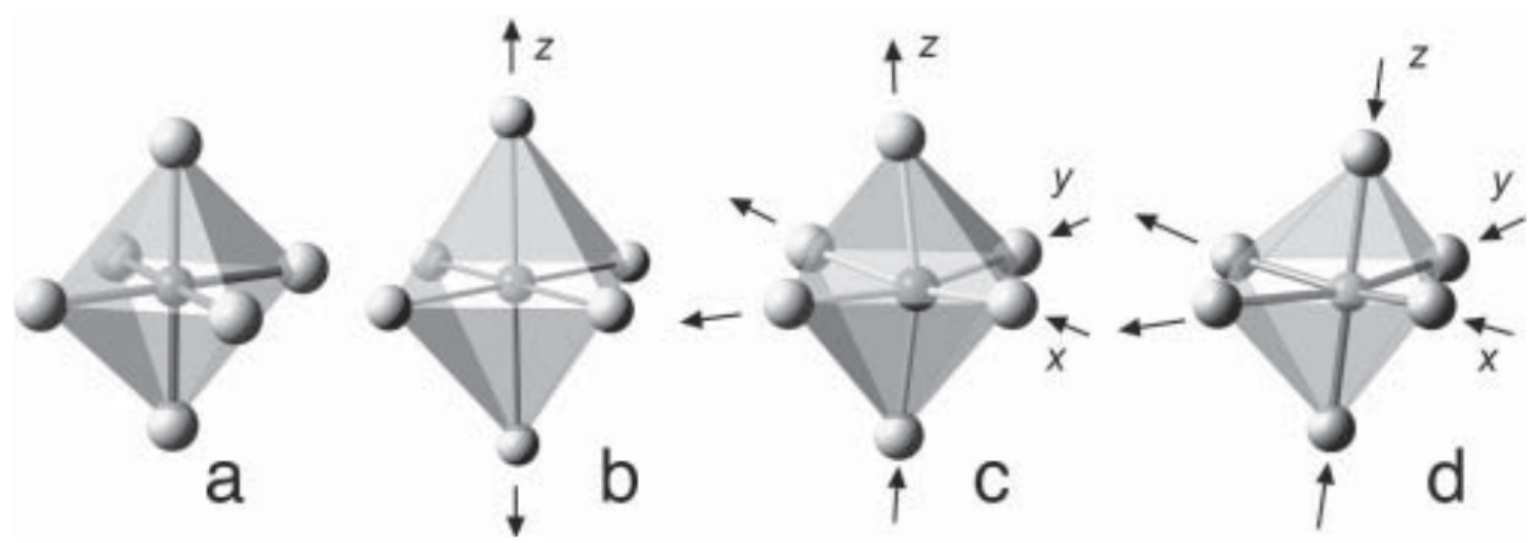

Figure 2.2.: $\mathrm{TiO}_{6}$ octahedra for (a) $\mathrm{TiO}$, (b) $\mathrm{r}-\mathrm{TiO}_{2}$, (c) $\mathrm{Ti}_{2} \mathrm{O}_{3}$ and (d) $\mathrm{Ti}_{4} \mathrm{O}_{7}$ and $\mathrm{Ti}_{5} \mathrm{O}_{9}$, respectively. Arrows indicate the distortions from the ideal octahedron, cf. (a), that appear in the corresponding phase. Reprinted with permission from Stoyanov et al. [22]. Copyright 2007 Mineralogical Society of America.

$\mathrm{TiO}$ has an ideal octahedral $\mathrm{TiO}_{6}$ arrangement [22], in which all $\mathrm{Ti}-\mathrm{O}$ and $\mathrm{O}-\mathrm{O}$ distances are the same and all $\mathrm{O}-\mathrm{Ti}-\mathrm{O}$ angles are $90^{\circ}$. The stoichiometric phases from $\mathrm{Ti}_{2} \mathrm{O}_{3}$ to $\mathrm{TiO}_{2}$, cf. fig. 2.1, reveal different distortions from the ideal octahedral arrangement [22]. Arrows in fig. 2.2 indicate the distortions. The $\mathrm{TiO}_{6}$ arrangement has a strong influence on the energetic states of the corresponding phase. The $\mathrm{r}-\mathrm{TiO}_{2}$ arrangement is discussed further in the following section.

Titanium dioxide $\left(\mathrm{TiO}_{2}\right)$ appears in three crystalline modifications [23], that are also referred to as 'polymorphs': rutile, anatase and brookite. Rutile is the thermodynamically stable polymorph of $\mathrm{TiO}_{2}$. Anatase is a metastable modification that transforms to rutile at elevated temperatures $(T \gtrsim 973 \mathrm{~K})$. Brookite is unstable.

$\mathrm{TiO}_{2}$ is known to be a nonstoichiometric compound [24] and is therefore often referred to as $\mathrm{TiO}_{2-x}$, with $x$ depending on the concentration of present lattice defects, but typically on the order of $10^{-2}$ to $10^{-4}$ [24]. It was shown recently that a metaldeficient oxide state can be formed in $\mathrm{r}^{-} \mathrm{TiO}_{2}$ by prolonged oxidation $[25,26]$. Thus, the more appropriate formula to describe the nonstoichiometry in $\mathrm{r}-\mathrm{TiO}_{2}$ is $\mathrm{Ti}_{1-x} \mathrm{O}_{2-y}$ [3], with $y<2 x$ (and $\left.y<10^{-2}\right)$.

\subsubsection{Symmetry and properties of $\mathrm{r}-\mathrm{TiO}_{2}$}

Rutile $\mathrm{TiO}_{2}$ has a rutile crystal structure, consisting of a tetragonal unit cell, as shown in fig. 2.3a. Some important properties of $\mathrm{r}-\mathrm{TiO}_{2}$ are summarized in tab. 2.1. A comprehensive summary of the properties of $\mathrm{r}-\mathrm{TiO}_{2}$ is given by Diebold [27] and by Grant [23]. 


\begin{tabular}{ll}
\hline \hline Rutile $\mathrm{TiO}_{2}$ & \\
\hline crystal system & tetragonal \\
crystal class (/point group) & ditetragonal bipyramidal \\
& $4 / \mathrm{m} 2 / \mathrm{m} 2 / \mathrm{m}[15]$ (Hermann-Mauguin notation) \\
& $\mathrm{D}_{4 \mathrm{~h}}[15]$ (Schoenflies notation) \\
& $\mathrm{P} 42 / \mathrm{mnm}$ (no. 136) [15] \\
space group & $a=b=(4.5929 \pm 0.0005) \AA[28]$ \\
lattice parameter & $c=(2.9591 \pm 0.0003) \AA[28]$ \\
& $4.2 \mathrm{eV} \mathrm{in}(110)$ direction $[29]$ \\
work function/ & $3.1 \mathrm{eV} \perp c[30]$ and $3.0 \mathrm{eV} \| c[31]$ \\
electron affinity & $0.106 \mathrm{~cm}^{2} \mathrm{~V}^{-1} \mathrm{~s}^{-1}[32]$ \\
band gap & \\
electron mobility ${ }^{2} \mu_{n}$ &
\end{tabular}

Table 2.1.: Properties of rutile titanium dioxide.

The $\mathrm{r}-\mathrm{TiO}_{2}$ unit cell and the corresponding octahedral arrangement is sketched in fig. 2.3. The $\mathrm{r}-\mathrm{TiO}_{2}$ unit cell and the corresponding (110) plane, which is highlighted in blue, are depicted in fig. 2.3a. The (110) plane is the crystal surface on which the palladium films are prepared. A detailed summary of the $\mathrm{r}-\mathrm{TiO}_{2}(110)$ surface is given by Diebold [27]. In the $\mathrm{r}-\mathrm{TiO}_{2}$ unit cell, the central $\mathrm{Ti}$ atom and its six next nearest $\mathrm{O}$ atoms ( 1 to 6 ) build up an octahedron (black lines), cf. fig. 2.3b. The $\mathrm{TiO}_{6}$ octahedron in $\mathrm{r}-\mathrm{TiO}_{2}$ is not ideal, cf. fig. 2.2b), and has distortions: One of the O-Ti-O axis is elongated by approximately $1.9 \%$ with respect to the other two axis, as indicated by the black arrow in fig. 2.3b. The Ti-O distance in the elongated direction is approximately $1.98 \AA\left(\mathrm{Ti}-\mathrm{O}^{1}=\mathrm{Ti}-\mathrm{O}^{2}\right)$ while the $\mathrm{Ti}-\mathrm{O}$ distance to the $\mathrm{O}$ atoms in the $\mathrm{TiO}_{4}$ plane is approximately $1.95 \AA\left(\mathrm{Ti}-\mathrm{O}^{3}=\mathrm{Ti}-\mathrm{O}^{4}=\mathrm{Ti}-\mathrm{O}^{5}=\mathrm{Ti}-\mathrm{O}^{6}\right)$. Additionally, the inner O-Ti-O angles in the $\mathrm{TiO}_{4}$ plane deviate from $90^{\circ}$, as shown in fig. 2.3c. The $\mathrm{O}^{3}-\mathrm{Ti}-\mathrm{O}^{4}$ angle is equal to the $\mathrm{O}^{5}-\mathrm{Ti}-\mathrm{O}^{6}$ angle and is approximately $81^{\circ}$. The $\mathrm{O}^{4}-\mathrm{Ti}-\mathrm{O}^{5}$ angle is equal to the $\mathrm{O}^{6}-\mathrm{Ti}-\mathrm{O}^{3}$ angle and is approximately $99^{\circ}$. This results in two different distances between the $\mathrm{O}$ atoms in the $\mathrm{TiO}_{4}$ plane and thus in a rectangular shape which is visible in fig. 2.3c. The $\mathrm{O}^{3}-\mathrm{O}^{4}$ and $\mathrm{O}^{5}-\mathrm{O}^{6}$ distances are approximately $2.54 \AA$ while the $\mathrm{O}^{4}-\mathrm{O}^{5}$ and $\mathrm{O}^{3}-\mathrm{O}^{6}$ distances are approximately $2.94 \AA$. Therefore, the octahedron has a orthorhombic distortion [35].

Typically, the octahedral arrangement is discussed with respect to the corresponding symmetry. The ideal octahedron $(\mathrm{TiO})$ has $\mathrm{O}_{\mathrm{h}}$ symmetry $[16,22]$. The discussed

\footnotetext{
${ }^{1}$ The band gap of $\mathrm{r}-\mathrm{TiO}_{2}$ has been measured with different techniques and samples in the past: Electrical conductivity, optical and thermopower experiments as well as theoretical calculations give a band gap between $3.0 \mathrm{eV}$ and $3.1 \mathrm{eV}$ [33]. Though, rarely, values above and below this range were also measured [33].

${ }^{2}$ While the electron mobility is almost not dependent of $T$, the hole mobility $\mu_{p}$ is a function of $T$ [32]. At $293 \mathrm{~K}$, the hole mobility is approximately $10^{-11} \mathrm{~cm}^{2} \mathrm{~V}^{-1} \mathrm{~s}^{-1}$ as extrapolated from measurements from Bak et al. performed between $1120 \mathrm{~K}$ and $1280 \mathrm{~K}$ [32].
} 


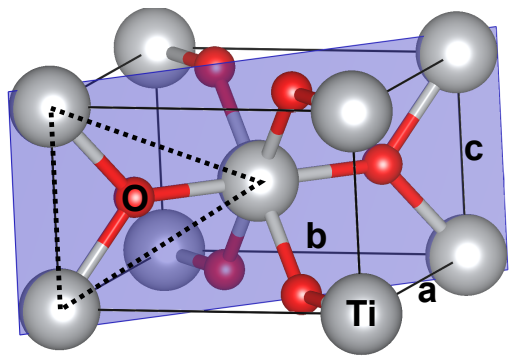

(a) Unit cell. A corresponding $\{110\}$ plane is indicated in blue. The $\mathrm{O}$ atoms (red) are each surrounded by three Ti atoms (gray). These three Ti atoms are arranged in a triangle around the corresponding $\mathrm{O}$ atom as indicated with the dashed lines.

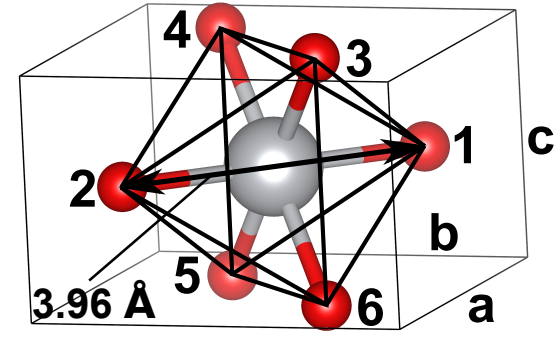

(b) Octahedron in the unit cell. The arrowheads indicate the elongated axis in [110] direction.

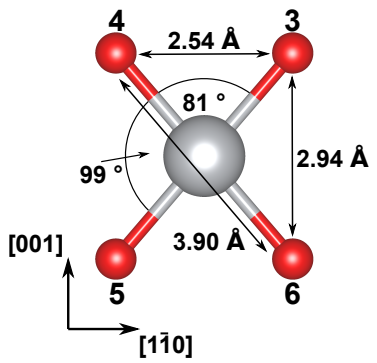

(c) $\mathrm{TiO}_{4}$ plane of the octahedron, top view in [110] direction. The $\mathrm{O}$ atoms arrange in a rectangle around the $\mathrm{Ti}$ atom.

Figure 2.3.: Structure of $\mathrm{r}-\mathrm{TiO}_{2}$. The unit cell is shown with the black frame. Numbers indicate the $\mathrm{O}$ atoms (in red) corresponding to the octahedron in the unit cell. The octahedron is orthorhombic distorted. Illustrated with the $3 \mathrm{D}$ visualization Software VESTA (v3.4) [34]. Coloring after CPK coloring of 'Jmol' [14].

distortions from the ideal octahedral shape lower the symmetry and, for $\mathrm{r}-\mathrm{TiO}_{2}$, the orthorhombic distortion yield a $\mathrm{D}_{2 \mathrm{~h}}$ symmetry $[22,35]$. It is important to note that the local symmetry of the Ti atoms should not be confused with the symmetry of the rutile lattice or of its corresponding crystal class which is $\mathrm{D}_{4 \mathrm{~h}}$ due to the tetragonal unit cell, cf. tab. 2.1. The local arrangement of the octahedron and its related symmetry influence the corresponding energetic states.

Fischer [36] calculated the energetic states in $\mathrm{TiO}$ with in a molecular-orbital (MO) picture. He assumed a $\mathrm{TiO}_{6}$ octahedron in $\mathrm{O}_{\mathrm{h}}$ symmetry and calculated the corresponding MO's. In the calculation, Fischer included the interaction of Ti $3 d, 4 s$ and $4 p$ states with $\mathrm{O} 2 s$ and $2 p$ states. The resulting MO diagram is shown in fig. 2.4a. The $\mathrm{O}_{\mathrm{h}}$ symmetry results in the typical $t_{2 g}-e_{g}$ crystal field splitting of the unoccupied density of states (DoS) as indicated in fig. 2.4a. As mentioned, the presented MO calculations of Fischer are valid for one undistorted $\mathrm{TiO}_{6}$ octahedron (with $\mathrm{O}_{\mathrm{h}}$ symmetry). In a solid, this octahedra occur periodically and thus the 'sharp' MO states broaden to narrow bands in the solid [36]. The MO diagram of Fischer, cf. fig. 2.4a can be qualitatively applied to $\mathrm{r}-\mathrm{TiO}_{2}$. The orthorhombic distortion of the $\mathrm{TiO}_{6}$ octahedron in $\mathrm{r}-\mathrm{TiO}_{2}$ and its corresponding $\mathrm{D}_{2 \mathrm{~h}}$ symmetry though, yield to an additional energy splitting [22,35], as shown in fig.2.4b. The $t_{2 g}$ and $e_{g}$ levels are no longer degenerated and split further, as determined by Brydson et al. [35]: The $t_{2 g}$ are split to $b_{2 g}, a_{g}$ and $b_{3 g}$ and the $e_{g}$ states are split to $a_{g}$ and $b_{1 g}$. A comparison of the energy splitting in $\mathrm{O}_{\mathrm{h}}$ and $\mathrm{D}_{2 \mathrm{~h}}$ symmetry is sketched in fig. 2.4b. Tab. 2.2 summarizes 


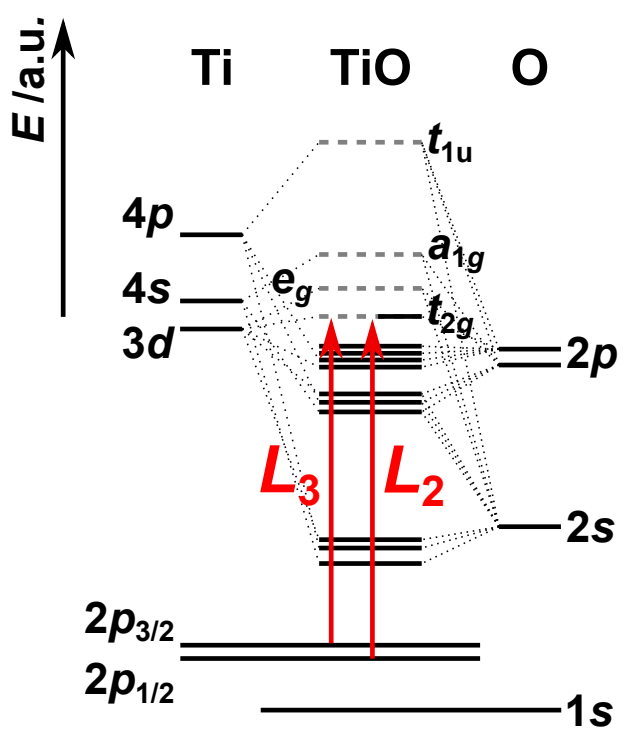

(a)

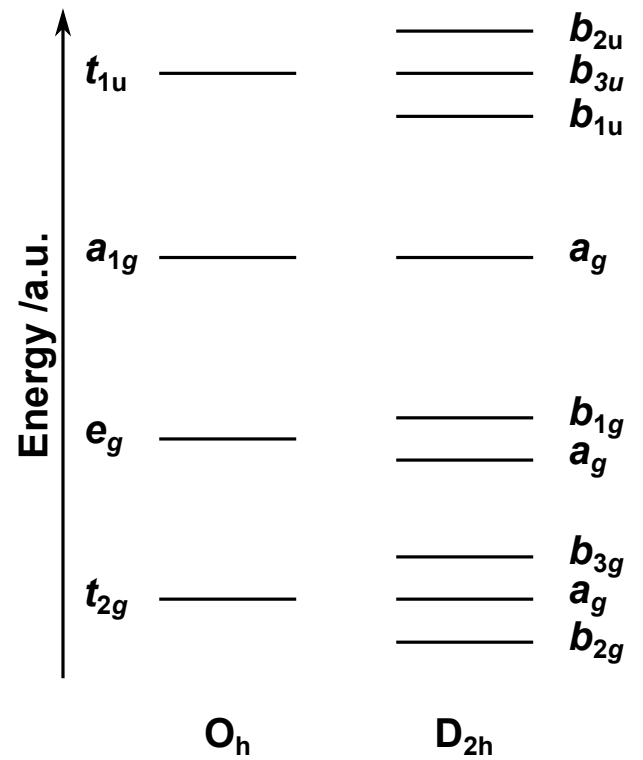

(b)

Figure 2.4.: (a) Schematic MO energy level diagram for TiO. Indicated are the $\mathrm{Ti}$ and $\mathrm{O}$ atom orbitals as well as the resulting molecular orbital in TiO. The Ti atom is here in the ideal octahedral oxygen environment $\left(\mathrm{O}_{\mathrm{h}}\right.$ symmetry), cf. fig. 2.2a. Unoccupied molecular orbitals are indicated with dashed lines. The dotted lines show the individual atomic orbitals that contribute to the corresponding molecular orbital. The Ti $L_{3}$ and $L_{2}$ line are indicated with red arrows and correspond to transitions from the Ti $2 p_{3 / 2}$ and $2 p_{1 / 2}$ orbital, respectively, to the unoccupied orbitals. This MO diagram can be qualitatively applied on $\mathrm{r}-\mathrm{TiO}_{2}$. In $\mathrm{TiO}$ the $t_{2 g}$ states are partially filled in $\mathrm{r}^{-\mathrm{TiO}_{2}}$ they are unoccupied. After Fischer [36]. (b) Schematic unoccupied MO energy levels in the ideal $\left(\mathrm{O}_{\mathrm{h}}\right)$, cf. fig. $2.2(\mathrm{a})$, and in the orthorhombic distorted, cf. fig. $2.3 \mathrm{~b},\left(\mathrm{D}_{2 \mathrm{~h}}\right)$ octahedral symmetry. The latter one is the appropriate one for r- $\mathrm{TiO}_{2}$. After Brydson et al. [35].

the relation between the $d$ orbitals and the corresponding symmetry labels of the ideal $\left(\mathrm{O}_{\mathrm{h}}\right)$ and orthorhombic distorted $\left(\mathrm{D}_{2 \mathrm{~h}}\right)$ octahedron.

The energy splitting introduced by the orthorhombic distorted octahedron $\left(\mathrm{D}_{2 \mathrm{~h}}\right)$ is important for spectroscopy since the additional energy splitting with can be seen on the $\mathrm{Ti} L$ edge in $\mathrm{r}-\mathrm{TiO}_{2}$ in electron energy loss spectroscopy (EELS) [22,35] or X-ray absorption spectroscopy [37]. In the following section, the Ti $L$ edge measured in EELS is discussed and related to the orthorhombic distorted octahedral symmetry in $\mathrm{r}-\mathrm{TiO}_{2}$. 


\begin{tabular}{ccc}
\hline \hline$d$-orbital & $\begin{array}{c}\text { symmetry } \\
\text { label } \mathrm{O}_{\mathrm{h}}\end{array}$ & $\begin{array}{c}\text { symmetry } \\
\text { label } \mathrm{D}_{2 \mathrm{~h}}\end{array}$ \\
\hline$d_{x z}$ & $t_{2 g}$ & $b_{2 g}$ \\
$d_{y z}$ & $t_{2 g}$ & $a_{g}$ \\
$d_{x y}$ & $t_{2 g}$ & $b_{3 g}$ \\
$d_{z^{2}}$ & $e_{g}$ & $a_{g}$ \\
$d_{x^{2}-y^{2}}$ & $e_{g}$ & $b_{1 g}$ \\
\hline \hline
\end{tabular}

Table 2.2.: $d$-orbitals and their symmetry label in an ideal octahedron $\left(\mathrm{O}_{\mathrm{h}}\right)$ and in an orthorhombic distorted octahedron $\left(\mathrm{D}_{2 \mathrm{~h}}\right)$, as in $\mathrm{r}-\mathrm{TiO}_{2}$. The elongated axis is chosen in the $z$ direction while $x$ and $y$ are lying in the $\mathrm{TiO}_{4}$ plane, cf. fig. 2.3b.

\subsubsection{The $\mathrm{Ti} L$ edge of $\mathbf{r}-\mathrm{TiO}_{2}$ in electron energy loss spectroscopy (EELS)}

As mentioned in the previous section, the symmetry and distortion of the $\mathrm{TiO}_{6}$ octahedron have influence on the unoccupied states. With EELS, electrons excited from core levels into this unoccupied states of the DoS can be detected, as indicated with the red arrows in fig. 2.4a. Thus, changes in the unoccupied states of the DoS can be identified using EELS. Following is a brief introduction into EELS and into the shape of the Ti $L$ edge in EELS.

A general introduction to EELS in a transmission electron microscope (TEM) is given by Williams and Carter [38, chap. 37 et seqq.], in more details by Egerton [39] or more compactly in a recent review by Egerton [40]. A simplified picture of EELS in TEM follows:

The primary electrons of a TEM can scatter inelastically within the sample. The corresponding energy loss $\Delta E$ and the corresponding transmitted electrons can be counted with a detector. The detected energy loss originates from the excitation of a (core) electron of the respective material to the unoccupied DoS. An EEL spectrum will therefore contain element specific ionization edges. The unoccupied DoS depends on the chemical surrounding of the specific atom, like the octahedral environment of Ti with $\mathrm{O}$ in titanium oxides. The energetic structure of the unoccupied DoS is thus directly connected to the energetic (fine) structure of the measured energy loss edge. This can yield to an energy loss near edge structure (ELNES), also referred to as fine structure oscillations, which can be seen in detail in the inset of fig. 2.5 for $\mathrm{r}^{-\mathrm{TiO}_{2}}$. Changes in the ELNES are typically correlated with a change in the unoccupied DoS and therefore in the chemical surrounding. It is important to note that the relation between the measured electron intensity in EELS and the underlying DoS is non trivial and given by the following proportionality [40, eq. 23]:

$$
J_{C} \propto(d f / d E) N(E)
$$

where $J_{C}$ is the core-loss intensity, $(d f / d E)$ is the atomic oscillator strength and $N(E)$ 
is the density of final states in the electron transition.

The Ti $L$ ionization edge is divided into three $L$ edges: $L_{3}, L_{2}$ and $L_{1}$. They respectively originate from electron excitations from the $2 p_{3 / 2}, 2 p_{1 / 2}$ and $2 s$ core orbitals to the unoccupied states, cf. red arrows in fig. 2.4a. For $\mathrm{r}-\mathrm{TiO}_{2}$, the $\mathrm{Ti} L$ structure is shown in fig. 2.5 using data from the Gatan EELS Atlas [41]. A typical EEL spectrum shows the amount of collected electrons per detector channel plotted against the energy loss $\Delta E$. The spectrum contains the pre-edge signal (cf. fig. 2.5), which originates from the plural-scattering background, followed by the actual Ti $L$ edge that is separated in the $L_{3}$ and $L_{2}$ edge. These edges overlap and possess a prominent ELNES which is shown in detail in the inset of fig. 2.5, or with higher energy resolution in fig. 2.6, and is a typical fingerprint for $\mathrm{r}-\mathrm{TiO}_{2}$ [22]. The Ti $L_{3,2}$ ELNES is followed by the common ionization cross section that contains additional contributions such as excitations to higher energetic states (cf. fig. 2.4) and extra plural-scattering on plasmons. The $\mathrm{O} K$ edge, starting at $\Delta E \approx 530 \mathrm{eV}$, is overlayed on this and is followed by the Ti $L_{1}$ edge starting at $\Delta E \approx 560 \mathrm{eV}$. Both the $\mathrm{O} K$ and $\mathrm{Ti} L_{1}$ edge have lower ionization cross-sections as is typical for excitations from $s$ orbitals [38]. Due to the poor signal, the $\mathrm{O} K$ and $\mathrm{Ti} L_{1}$ edges are not suitable for precise measurements in the immediate vicinity of the $\mathrm{Pd} / \mathrm{r}-\mathrm{TiO}_{2}$ interface as required for this work.

Stoyanov et al. [22] summarized the recent knowledge about the Ti $L$ edge in EELS for different titanium oxides. They interpret the shape of the Ti $L$ ELNES in the context of the MO theory based on the octahedral arrangement of $\mathrm{O}$ around the $\mathrm{Ti}$ atom. Their terminology of labelling the $\mathrm{Ti} L_{3,2}$ edge fine structure is used as indicated in fig. 2.6. The Ti $L$ fine structure was also investigated by Kucheyev et al. [37] with $\mathrm{x}$-ray absorption near-edge structure (XANES) spectroscopy. It is important to note that every titanium oxide phase (between $\mathrm{TiO}$ and $\mathrm{TiO}_{2}$ ), cf. fig. 2.1, has it own $\mathrm{Ti} L$ ELNES shape [22]. Therefore, the ELNES serves as a fingerprint of the present $\mathrm{Ti}_{\mathrm{y}} \mathrm{O}_{\mathrm{x}}$ phase. The shape of the ELNES is thus an important indication of which titanium oxide phase is present. Small changes in the $\mathrm{r}-\mathrm{TiO}_{2}$ ELNES are expected due to hydrogen loading. Not expected are phase transitions to stoichiometric phases below $\mathrm{r}-\mathrm{TiO}_{2}$, thus the general shape of the ELNES is not expected to change as it would by the formation of under stoichiometric phases [22]. A detailed understanding of the Ti $L$ ELNES is therefore of importance and is introduced in the following.

The $L_{3,2}$ ELNES is made of two 'white lines' (intense peaks) in the $L_{3}$ and $L_{2}$ edges. The white lines are labeled with $a, b, c$ and $d$ in order of appearance from lower to higher energy losses. The MO theory predicts the splitting of the $L$ edges in $a$ and $b$ for the $L_{3}$ and in $c$ and $d$ for the $L_{2}$ edge as a result of the octahedral arrangement, as already discussed in the previous section. The splitting is due to the crystal (or ligand) field in $t_{2 g}$ and $e_{g}$. Thus, the $a$ and $c$ white line correspond to $t_{2 g}$ states and the $b$ and $d$ white line correspond to $e_{g}$ states. A measurement with high energy resolution of the Ti $L$ ELNES can be found with the labeled white lines in fig. 2.6. Brydson et al. [42] reported a splitting in the $b$ white line in EELS experiments on the $\mathrm{Ti} L_{3}$ edge. The splitting between $b$ and $b^{\prime}$ is roughly between $0.8 \mathrm{eV}[22]$ and $0.9 \mathrm{eV} \mathrm{[42].} \mathrm{It} \mathrm{corresponds}$ 


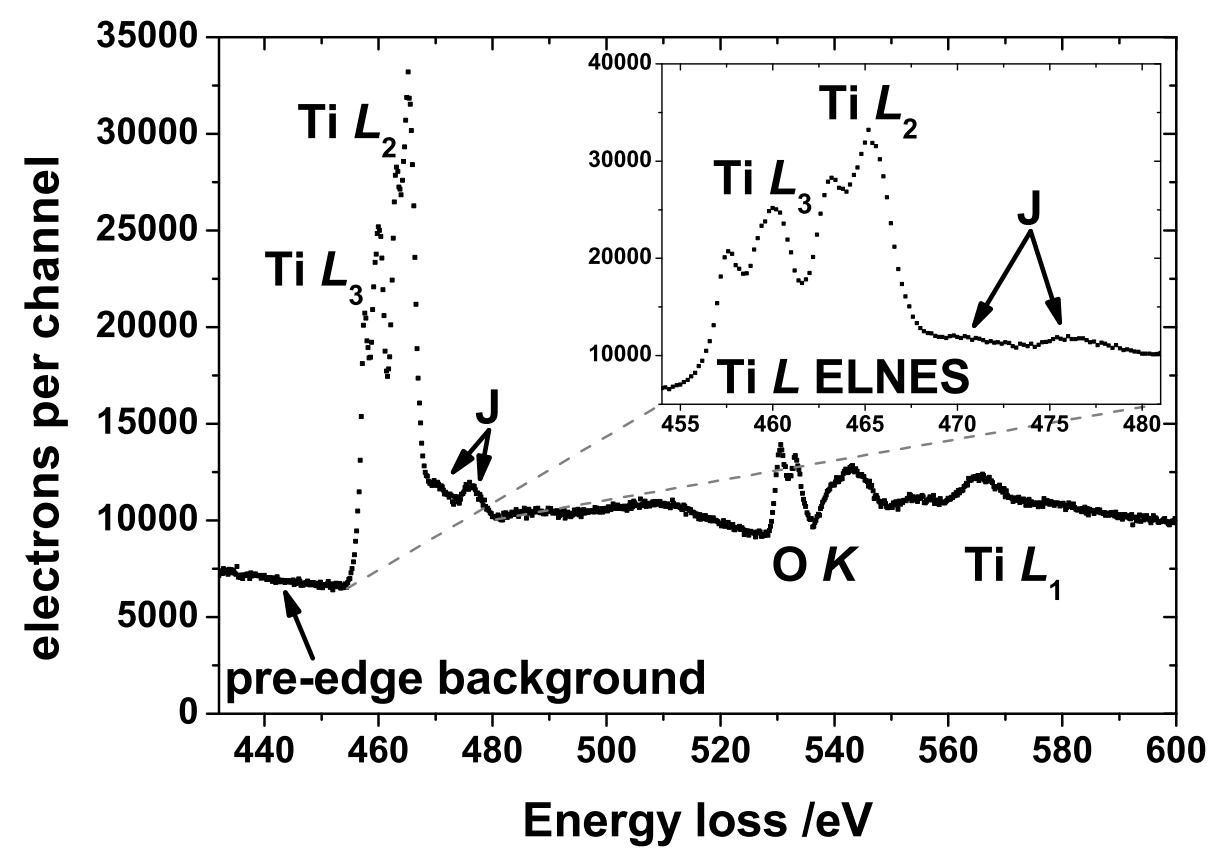

Figure 2.5.: Shown is the amount of collected electrons per detector channel against the energy loss of the corresponding electrons. The displayed energy loss region contains the general structure of the $\mathrm{Ti} L$ and $\mathrm{O} K$ edge for $\mathrm{r}-\mathrm{TiO}_{2}$. The Ti $L_{3}$ and $L_{2}$ edge both reveal an ELNES, visible in the magnified inset graph. Post edge features are indicated with J. The shown EELS data were taken from the Gatan EELS Atlas [41].

to a splitting of the $e_{g}$ states which has its origin in the octahedral distortion in $\mathrm{r}-\mathrm{TiO}_{2}$ (cf. chap. 2.1) $[22,35,42]$. Thus, the two $e_{g}$ states can be referred to as $a_{g}$ and $b_{1 g}$ in the context of the MO theory (cf. tab. 2.5 and fig. 2.4b). The $d$ white line in the $L_{2}$ edge also shows the splitting but it is a lot weaker [37]. In EELS, the splitting of the $d$ white line is typically not visible. It was observed in highly resolved XANES measurements by Kucheyev et al. [37]. This originates from the stronger broadening of the $L_{2}$ edge ( $c$ and $d$ white line) due to a reduced life time of the core hole that makes the splitting less obvious [37,42].

More recent findings of Cheynet et al. [43] reveal an even stronger splitting in the $b$ white line into three peaks and thus of the related $e_{g}$ states. Cheynet et al. [43] used a "multichannel multiple scattering theory approach" to simulate the Ti $L$ ELNES and explain the observed additional splitting in the $b / b^{\prime}$ white line. Based on their calculations Cheynet et al. [43] suggested that "long-range band structure effects" can yield this additional splitting $[43,44]$. Thus, the up-to-now discussed MO theory and the related ligand/crystal field splittings cannot explain the new findings and additional long-range effects need to be considered [37], as for example higher order coordination shells. This additional splitting of the $b$ white line in three peaks has not 


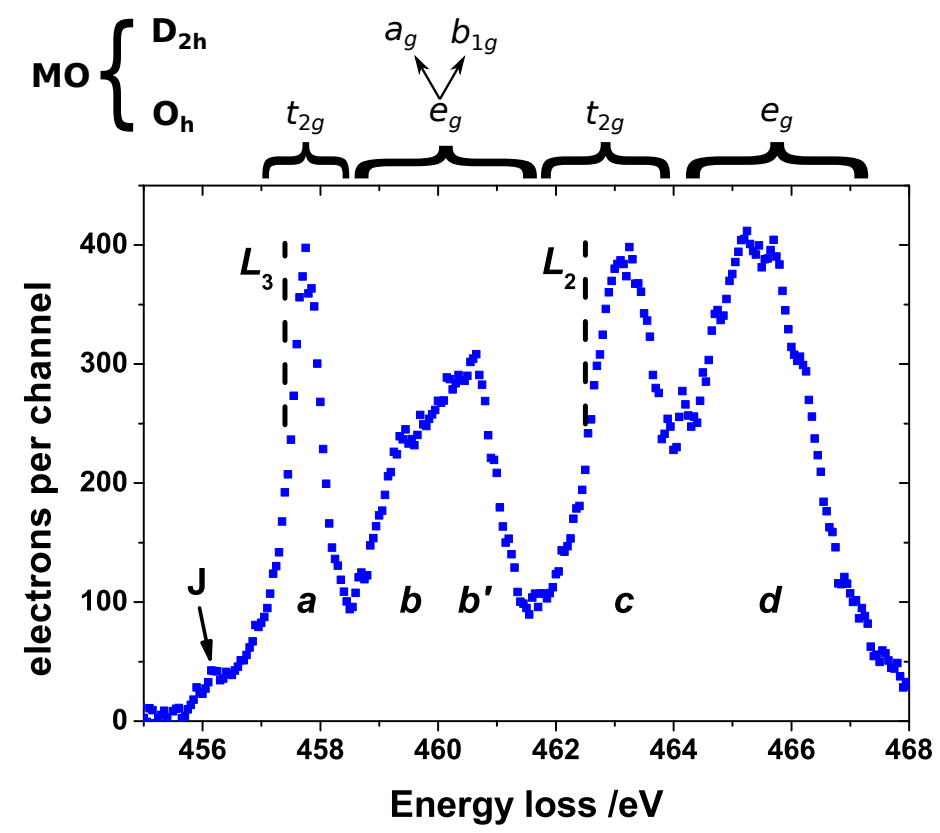

Figure 2.6.: Example of a measured Ti $L_{2,3}$ ELNES (blue) of $\mathrm{r}-\mathrm{TiO}_{2}$. The $L_{3}$ and $L_{2}$ edge onset as well as the four white lines $a, b / b^{\prime}, c$ and $d$ and a pre-edge feature $\mathrm{J}$ are indicated. The separation of the white lines can be understood with MO theory, cf. fig.2.4, by considering the symmetry of an ideal $\left(\mathrm{O}_{\mathrm{h}}\right)$ and orthorhombic distorted $\left(\mathrm{D}_{2 \mathrm{~h}}\right)$ $\mathrm{TiO}_{6}$ octahedron. This is indicated in the figure: The $a$ and $c$ white line correspond to $t_{2 g}$-like and the $b / b^{\prime}$ and $d$ white line correspond to $e_{g}$-like states, thus the typical crystal field splitting results from an ideal octahedral environment of the $\mathrm{O}$ atoms around the Ti atom. The further splitting of the $b / b^{\prime}$ white line, and thus of the $e_{g^{-}}$ like states, is due to the orthorhombic distortion in $\mathrm{r}-\mathrm{TiO}_{2}$, cf. fig. 2.3, and correspond to the $a_{g}$ and $b_{1 g}$-like states, respectively, cf. fig. $2.4 \mathrm{~b}$.

been observed in highly resolved XANES measurements.

In this work, no additional splittings were found like those reported by Cheynet et al. [43]. Here, the MO theory suffices to picture the splitting of the $b$ white line in the Ti $L_{3}$ edge of $\mathrm{r}-\mathrm{TiO}_{2}$.

Interesting, no splitting has been found for the $t_{2 g}$ states, even in the high resolved ELNES of Cheynet et al. [43] or XANES from Kucheyev et al. [37]. This finding can be explained in a simplified picture: the $d$ orbitals involved in the $t_{2 g}$ states, which form $\pi$ like bonds, have weaker overlap. Therefore their modification by the octahedral distortion is small if compared to the $d$ orbitals involved in the formation of the $e_{g}$ states, which form $\sigma$ like bonds. The $d$ orbitals and their arrangement in an octahedron are shown in the appendix in fig. A.1.

The $\mathrm{Ti} L_{3,2}$ edge of $\mathrm{r}-\mathrm{TiO}_{2}$ reveals two additional features: intensity variations are found in front of the $a$, cf. $\mathrm{J}$ in fig. 2.6, and behind the $d$ white line, cf. $\mathrm{J}$ in fig. 2.5. 
The latter can be understood in the context of MO theory by transitions from the $\mathrm{Ti}$ $2 p$ states to the $t_{1 u}$ stats [37], cf. fig. 2.4b. The origin of the peaks in front of the $a$ white line ${ }^{3}$ is still under debate $[22,37]$. Both, the ELNES features in front of the $a$ and behind the $d$ white line are not of importance for this work.

Brydson et al. [35] found identical shapes of the $\mathrm{Ti} L$ ELNES for $\mathrm{r}-\mathrm{TiO}_{2}$ single crystals that had different orientations of the crystals $c$ axis to the incident electron beam. Therefore the crystal orientation should have no influence on the Ti $L$ ELNES of the $\mathrm{r}-\mathrm{TiO}_{2}$ single crystals measured in the EELS experiments.

In summary, the $\mathrm{Ti} L_{3,2}$ ELNES of $\mathrm{r}-\mathrm{TiO}_{2}$ can be understood in the context of MO theory, cf. fig. 2.6. The octahedral arrangement of $\mathrm{O}$ around the Ti atoms results in a corresponding energy splitting in the related unoccupied DoS. The appearance of four white line $a$ and $b / b^{\prime}$ in the $L_{3}$ edge and $c$ and $d$ in the $L_{2}$ edge can be explained by the crystal field splitting introduced by an ideal octahedron $\left(\mathrm{O}_{\mathrm{h}}\right.$ symmetry). Thus $a$ and $c$ correspond to $t_{2 g}$-like and $b / b^{\prime}$ and $d$ correspond to $e_{g}$-like states. The additional splitting in the $b$ and $b^{\prime}$ result from the orthorhombic distorted octahedron $\left(\mathrm{D}_{2 \mathrm{~h}}\right.$ symmetry) present in $\mathrm{r}-\mathrm{TiO}_{2}$, cf. fig. 2.3 and 2.4 .

\subsubsection{Electron beam damage}

The bombardment of a sample with electrons in a TEM can result in the atomic displacement of particular atoms from their position in the crystal lattice, which is also known as 'knock-on damage'. The threshold displacement energy $E_{d}$ is approximately $20 \mathrm{eV}$ for $\mathrm{O}$ and $73 \mathrm{eV}$ for $\mathrm{Ti}$ in $\mathrm{r}-\mathrm{TiO}_{2}$ [45]. For each $E_{d}$ the maximum energy of the incident electrons $E_{0}$ in a TEM can be calculated via [46, eq. 2], assuming a central collision of the electron with a nucleus:

$$
E_{d}=\frac{E_{0}\left(1.02+E_{0} / 10^{6}\right)}{465.7 \cdot A}
$$

where $A$ is the atomic mass number.

Equ. 2.2 yields to an $E_{0}$ of approximately $130 \mathrm{keV}$ for $\mathrm{O}$ and of approximately $864 \mathrm{keV}$ for Ti. This shows that primarily $\mathrm{O}$ is displaced due to high-angle elastic scattering [46] with oxygen nuclei in $\mathrm{r}-\mathrm{TiO}_{2}$ in a $300 \mathrm{keV}$ electron beam.

Thus, atomic displacement by the $300 \mathrm{keV}$ primary electrons of the used ETEM is possible for O but unlikely for Ti. Therefore it is important to keep the electron dose to a minimum to prevent oxygen displacement.

\footnotetext{
${ }^{3} \mathrm{~A}$ "strong interaction between poorly screened $3 d$ electrons and the $2 p$ core hole" as well as "sharp features in the (one-electron) Ti $3 d$ partial DoS" are discussed [37].
} 


\subsubsection{Intrinsic defects $\mathrm{r}-\mathrm{TiO}_{2}$}

$\mathrm{R}-\mathrm{TiO}_{2}$ is a semiconductor with a measured band gap of approximately $3 \mathrm{eV}$ (cf. tab. 2.1) that can be modified by intrinsic defects. These defects allow it to achieve $n$ - or $p$-type conductivity. The $n$-type conductivity is typically associated with $\mathrm{O}$ vacancies $\left(\mathrm{V}_{\mathrm{O}}\right)$ and $\mathrm{Ti}$ interstitials $\left(\mathrm{Ti}_{\mathrm{i}}\right)[21]$ while the $p$-type conductivity is associated with $\mathrm{Ti}$ vacancies $\left(\mathrm{V}_{\mathrm{Ti}}\right)[25]$. The defects introduce states within the band gap that increase the number of charge carriers and therefore increase the conductivity of the material. In addition, the reactivity in the water oxidation reaction is also increased by the presence of defects $[3,9]$. Hence, the defects play a crucial role on the properties of $\mathrm{r}-\mathrm{TiO}_{2}$.

Diebold [27] summarized that $\mathrm{r}-\mathrm{TiO}_{2}$ can be reduced easily: The reduction produces defects of $n$-type nature. A reduced crystal exhibits an increased conductivity and a color change starting from transparent (not reduced) to light (reduced) and dark blue (strongly reduced). A reduced crystal contains $\mathrm{V}_{\mathrm{O}}, \mathrm{Ti}_{\mathrm{i}}$ and even planar defects (e.g. crystallographic shear planes [47]). $\mathrm{V}_{\mathrm{O}}$ travels via site-exchange and vacancy diffusion mechanism through the crystal. The diffusion of excess Ti happens by the interstitial mechanism and occurs preferentially along the $c$-axis of the crystal.

Interstitial oxygen $\left(\mathrm{O}_{\mathrm{i}}\right)$ is typically not considered in $\mathrm{r}-\mathrm{TiO}_{2}$ and thus is not discussed in older works, for example in [21]. O $\mathrm{O}_{\mathrm{i}}$ was mainly studied theoretically [6, 48-50]. These theory works indicate that $\mathrm{O}_{\mathrm{i}}$ mainly forms under $\mathrm{O}$-rich conditions as peroxide defect [6]. In O-rich conditions, $\mathrm{V}_{\mathrm{Ti}}$ has been found to be the dominating defect [6, $48,49]$. Hence, $\mathrm{O}_{\mathrm{i}}$ is typically not considered in the defect chemistry of $\mathrm{r}-\mathrm{TiO}_{2}$.

Antisite defects, such as $\mathrm{Ti}_{\mathrm{O}}$ and $\mathrm{O}_{\mathrm{Ti}}$, are also conceivable and were treated in first-principles calculations [49]. The simulated antisite defects turned out to exhibit high formation energies and are therefore of minor importance when compared to other intrinsic defects. Density functional theory (DFT) was also applied $[48,50]$ on Frenkel, anti-Frenkel and Schottky defect complexes. These exhibited high formation energies [48] and are not expected in any significant concentration.

The addressed theoretical works [48-50] found $\mathrm{V}_{\mathrm{O}}, \mathrm{Ti}_{\mathrm{i}}$ and $\mathrm{V}_{\mathrm{Ti}}$ to be the dominant intrinsic defects in $\mathrm{r}-\mathrm{TiO}_{2}$ in the experimentally accessible temperature and oxygen pressure range. This suggests that the intrinsic defect chemistry is dominated by these defects.

The intrinsic defect chemistry of a high purity $\mathrm{r}-\mathrm{TiO}_{2}$ single crystal (acceptor type elements $\lesssim 32 \mathrm{ppm}$ [30]) has been studied by Nowotny et al. [24,26, 30,51] via electrical conductivity and thermoelectric power measurements. In their measurements, Nowotny et al. studied the electrical conductivity $\sigma$ [30], cf. fig. 2.8, and the thermoelectric power $S$ [51] as a function of temperature $T$ and oxygen activity ${ }^{4} p\left(\mathrm{O}_{2}\right)$. They pretreated the $\mathrm{r}-\mathrm{TiO}_{2}$ single crystal in an argon (Ar) atmosphere at elevated

\footnotetext{
${ }^{4}$ Oxygen partial pressure that is measured by a zirconia-based electrochemical oxygen probe [30].
} 
temperatures, resulting in the presence of a constant concentration of $\mathrm{V}_{\mathrm{Ti}}$. Therefore, their results include effects of $\mathrm{V}_{\mathrm{Ti}}$ as an acceptor type defect ${ }^{5}$.

To describe the defect chemistry in undoped $\mathrm{r}-\mathrm{TiO}_{2}$, Nowotny et al. evaluated their results in a defect disorder model. In this model the following equations describe the three considered intrinsic defects $\left(\mathrm{V}_{\mathrm{O}}, \mathrm{Ti}_{\mathrm{i}}, \mathrm{V}_{\mathrm{Ti}}\right)$ in Kröger-Vink notation ${ }^{6}[3,30]$ :

$$
\begin{aligned}
\mathrm{O}_{\mathrm{O}}^{x} & \rightleftharpoons \mathrm{V}_{\mathrm{O}}^{2+}+2 e^{-}+\frac{1}{2} \mathrm{O}_{2} \\
2 \mathrm{O}_{\mathrm{O}}+\mathrm{Ti}_{\mathrm{Ti}} & \rightleftharpoons \mathrm{Ti}_{\mathrm{i}}^{3+}+3 e^{-}+\mathrm{O}_{2} \\
2 \mathrm{O}_{\mathrm{O}}+\mathrm{Ti}_{\mathrm{Ti}} & \rightleftharpoons \mathrm{Ti}_{\mathrm{i}}^{4+}+4 e^{-}+\mathrm{O}_{2} \\
\mathrm{O}_{2} & \rightleftharpoons 2 \mathrm{O}_{\mathrm{O}}+\mathrm{V}_{\mathrm{Ti}}^{4-}+4 h^{+} \\
\mathrm{nil} & \rightleftharpoons e^{-}+h^{+}
\end{aligned}
$$

These equations result in the lattice charge neutrality condition [52]:

$$
2\left[\mathrm{~V}_{\mathrm{O}}^{2+}\right]+3\left[\mathrm{Ti}_{\mathrm{i}}^{3+}\right]+4\left[\mathrm{Ti}_{\mathrm{i}}^{4+}\right]+p=n+4\left[\mathrm{~V}_{\mathrm{Ti}}^{4-}\right]
$$

Here [ ] indicated the concentration of the corresponding quantity and $n$ and $p$ represent the concentrations of electrons and holes, respectively. Based on these equations, Nowotny et al. $[3,30]$ defined different regimes. In each regime, one of intrinsic defect species dominates the conductivity. This is the base of Nowotny et al.'s defect disorder model $[3,30]$. The regimes with the intrinsic defect concentrations, indicated with [ ], are illustrated qualitatively against the oxygen activity in fig. 2.7. The concentrations of $\mathrm{Ti}_{\mathrm{i}}^{4+}$ are lower than these of the trivalent Ti interstitials in a wide $p\left(\mathrm{O}_{2}\right)$ range [24]. Therefore they are typically not considered in the defect chemistry of $\mathrm{r}-\mathrm{TiO}_{2}$. Only for very low oxygen activity is the 'extremely reduced regime' present. In this regime, the dominating defects are trivalent $\mathrm{Ti}$ interstitials $\left(\mathrm{Ti}_{\mathrm{i}}^{3+}\right)$ that are compensated by electrons $\left(e^{-}\right)$. With increasing $p\left(\mathrm{O}_{2}\right), \mathrm{r}_{-} \mathrm{TiO}_{2}$ is in the 'strongly reduced regime'. Here, doubly positively charged $\mathrm{V}_{\mathrm{O}}\left(\mathrm{V}_{\mathrm{O}}^{2+}\right)$ dominate and are compensated by electrons. Further increasing $p\left(\mathrm{O}_{2}\right)$ brings $\mathrm{r}-\mathrm{TiO}_{2}$ to the 'reduced regime', which is the relevant regime for this work, and, by a $n-p$ transition, to the 'oxidized regime'. In these regimes, the ionic charge compensation is given by $\left[\mathrm{V}_{\mathrm{O}}^{2+}\right]=2\left[\mathrm{~V}_{\mathrm{Ti}}^{4-}\right][30]$ and the predominant defect is $\mathrm{V}_{\mathrm{O}}$. While in the reduced regime, the conductivity is determined by electrons, in the oxidized regime, the conductivity is determined by holes $\left(h^{+}\right)$. The point where minimal electrons and holes are present defines the $n-p$ transition (red line in fig. 2.7); here electrons and holes are in equilibrium. For even higher $p\left(\mathrm{O}_{2}\right)$,

\footnotetext{
${ }^{5}$ This is an important difference to the performed treatment of the $\mathrm{r}-\mathrm{TiO}_{2}$ crystals. Here, the selected $T$ and $p\left(\mathrm{O}_{2}\right)$ ranges do not allow for the formation of significant concentrations of $\mathrm{V}_{\mathrm{Ti}}$. Later on it is discussed what deviations occur without the presence of acceptor type defects (as Ti vacancies).

${ }^{6}$ In Kröger-Vink notation the following abbreviations are used: - indicates a negative and + a positive charge, $\mathrm{X}_{\mathrm{Y}}$ indicates the defect $\mathrm{X}$ sitting on the sublattice of species $\mathrm{Y}$, e represents an electron and $h$ a hole.
} 


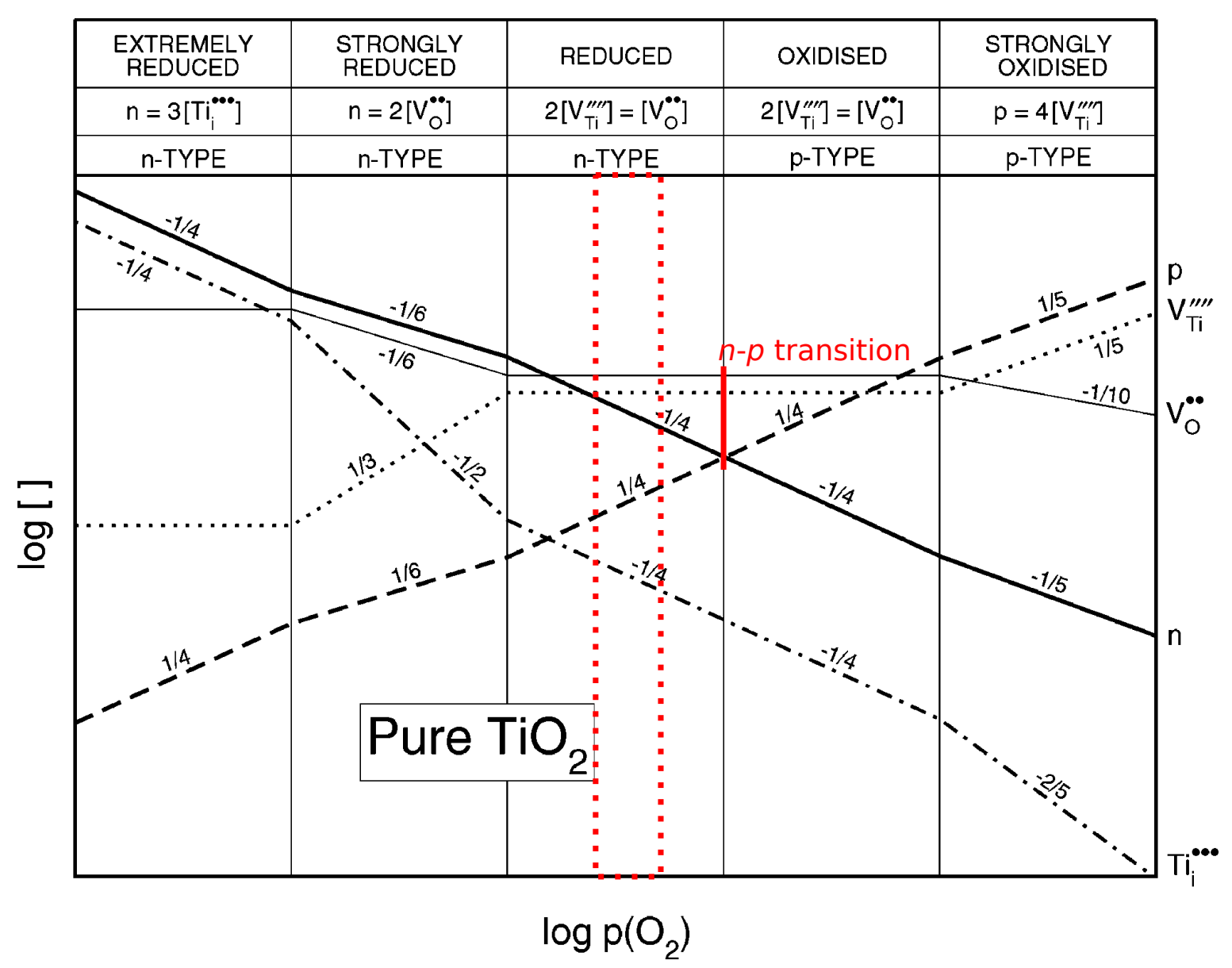

Figure 2.7.: The concentration [ ] of different species in a $\mathrm{r}-\mathrm{TiO}_{2}$ single crystal is illustrated against the oxygen activity $p\left(\mathrm{O}_{2}\right)$. Regimes are defined in which one intrinsic defect species is dominantly present. The $n-p$ transition is indicated with the red line and the pressure range of the performed annealing experiments with the red dotted rectangle. Adapted with permission from [3]. Copyright 2008 American Chemical Society.

the 'strongly oxidized regime' appears in which tetravalent $\mathrm{V}_{\mathrm{Ti}}\left(\mathrm{V}_{\mathrm{Ti}}^{4-}\right)$ dominates and is compensated by holes.

The qualitative picture of the oxygen activity regimes derived by Nowotny et al. [3], cf. fig.2.7, can be applied on real $\mathrm{r}-\mathrm{TiO}_{2}$ crystals, which can also contain relatively high concentrations of impurities. Conductivity measurements of such $\mathrm{r}-\mathrm{TiO}_{2}$ crystals can allow the elucidation of the corresponding defect disorder diagram.

This qualitative picture can be transferred to real measurements of the conductivity on a $\mathrm{r}-\mathrm{TiO}_{2}$ single crystal as shown in fig. 2.8 [30]. They measured the conductivity as a function of $T$ and $p\left(\mathrm{O}_{2}\right)$ in the range from $T=1073 \mathrm{~K}$ to $T=1323 \mathrm{~K}$ and $p\left(\mathrm{O}_{2}\right)=10^{-14} \mathrm{~Pa}$ to $p\left(\mathrm{O}_{2}\right)=10^{5} \mathrm{~Pa}$. The strongly reduced regime is found up to 


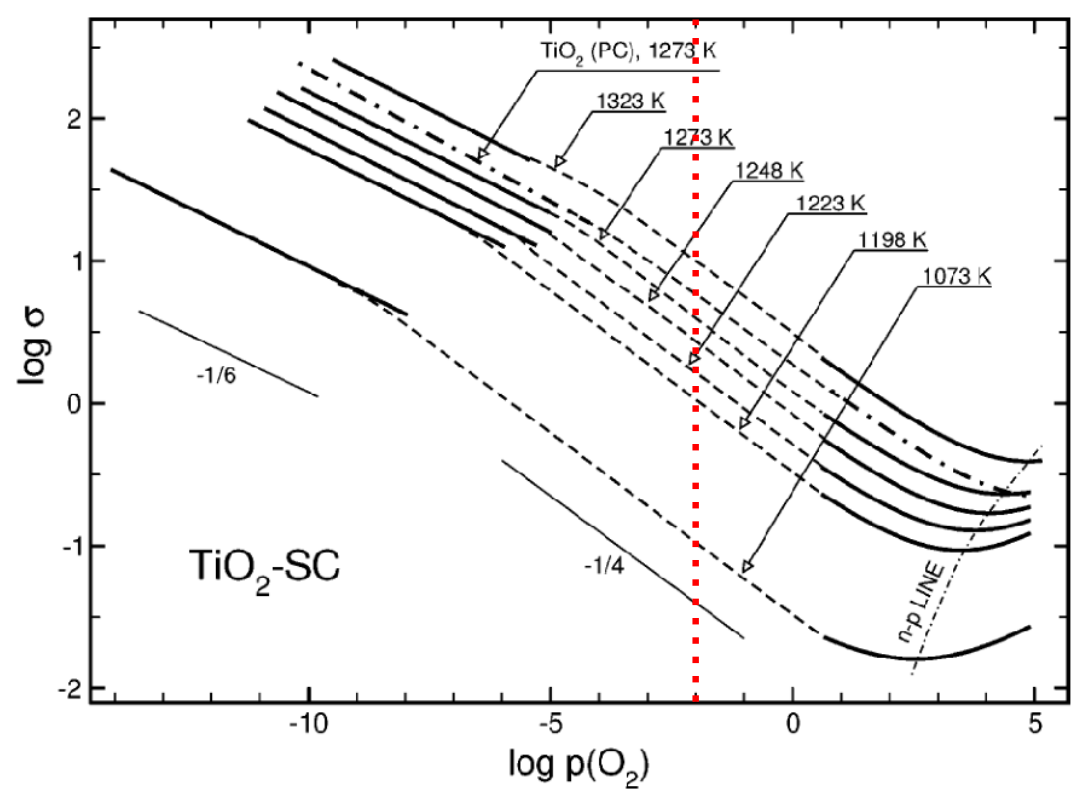

Figure 2.8.: The electrical conductivity $\sigma$ of a $\mathrm{r}-\mathrm{TiO}_{2}$ single crystal is shown against the oxygen activity $p\left(\mathrm{O}_{2}\right)$ for different temperatures $T$. The $n$ - $p$ transition line depends on $T$ and is indicated in the figure. Here, $\sigma$ is given in $\Omega^{-1} \mathrm{~m}^{-1}$ and $p\left(\mathrm{O}_{2}\right)$ in Pa. The red dotted box indicates the pressure range of the performed annealing experiments on the $\mathrm{r}-\mathrm{TiO}_{2}$ crystals. Adapted with permission from Nowotny et al. [30]. Copyright 2006 American Chemical Society.

a pressure of approximately $p\left(\mathrm{O}_{2}\right)=10^{-5} \mathrm{~Pa}$, further increasing $p\left(\mathrm{O}_{2}\right)$ up to $10^{2} \mathrm{~Pa}$ reveals the reduced regime. Depending on $T$ the reduced regime changes into the oxidized regime by going through the $n-p$ transition. The $n-p$ transition depends on $T$ and therefore gives a $n-p$ line, cf. fig. 2.8. The presence of the $n-p$ transition makes $\mathrm{r}-\mathrm{TiO}_{2}$ an amphoteric semiconductor [30]. As Nowotny et al. comment, the extremely reduced regime is not accessible with the investigated activity and temperature range. This regime is thus difficult to form experimentally. An additional trend is visible: For lower $T$ the minimal conductivity at the $n-p$ transition point decreases. Thus, lower temperatures yield to less defects present in the $\mathrm{r}-\mathrm{TiO}_{2}$ crystal. The occurrence of the $n-p$ transition in the selected temperature and $\mathrm{O}$ pressure range happens due to the presence of acceptor type defects like $\mathrm{V}_{\mathrm{Ti}}$.

$\mathrm{R}$ - $\mathrm{TiO}_{2}$ crystals without acceptor type defects do not show a $n$ - $p$ transition under these conditions [53,54]. Carpentier et al. [54] have studied chromium (Cr)-doped $\mathrm{r}-\mathrm{TiO}_{2}$ single crystals with different amount of $\mathrm{Cr}$. They discovered that the undoped $\mathrm{r}-\mathrm{TiO}_{2}$ crystal does not reveal a $n-p$ transition in the accessible pressure range, but Cr-doped crystals with concentrations at 1 at $\%$ and above do show the $n-p$ transition. Fig. 2.9 shows the conductivity vs $p\left(\mathrm{O}_{2}\right)$ measurements of an undoped $\mathrm{r}-\mathrm{TiO}_{2}$ crystal as well as of Cr-doped crystals [54]. The undoped crystal revealed a decreasing conduc- 


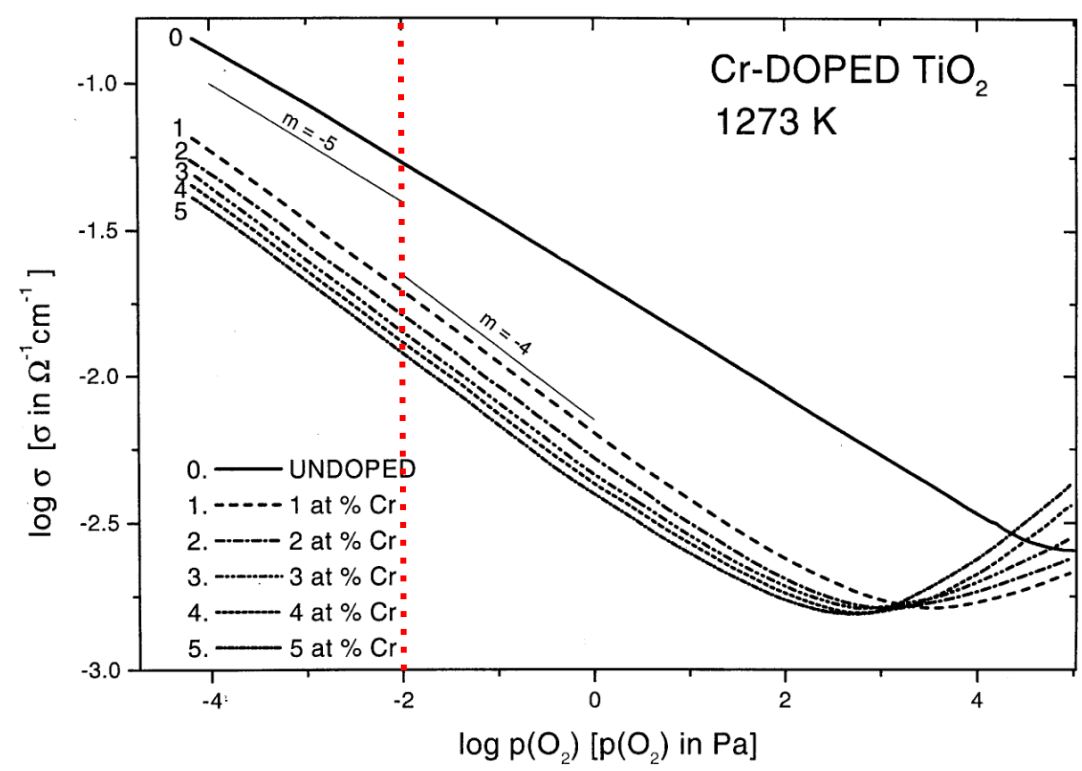

Figure 2.9.: The conductivity $\sigma$ as a function of the oxygen partial pressure $p\left(\mathrm{O}_{2}\right)$. The electrical conductivity $\sigma$ is shown for a undoped $\mathrm{r}^{-\mathrm{TiO}_{2}}$ crystal and for a doped crystals with Chromium (Cr) concentrations ranging from 1 at\% to 51 at\%. While the undoped crystal does not reveal a $n-p$ transition the Cr-doping introduces acceptor type defects resulting in the oxidized regime with $p$-type conductivity. Around $10^{-2} \mathrm{~Pa}$ (red dotted line) all crystal reveal $n$-type conductivity. Adapted with permission from Bak et al. [33] and from Carpentier et al. [54]. Copyright 2003 Elsevier and 1986 EDP Sciences.

tivity until the maximum of $p\left(\mathrm{O}_{2}\right)$ of approximately $10^{5} \mathrm{~Pa}$, cf. fig. 2.9. A comparable behavior is expected for the undoped $\mathrm{r}-\mathrm{TiO}_{2}$ crystals used. With a $\mathrm{Cr}$ concentration between $1 \%$ and $5 \%$ the $n-p$ transition was observed in the investigated oxygen activity range, cf. fig. 2.9. The $p\left(\mathrm{O}_{2}\right)$ of the $n$ - $p$ transition was found to depended on the Cr concentration [54]. The appearance of the $n-p$ transition was explained with an increasing concentration of acceptor-type defects with the increasing Cr content [33]. The acceptor defects can supply sufficient holes at high $p\left(\mathrm{O}_{2}\right)$ to result in a $p$ conductivity of the whole crystal. This indicates that a $p$-type conductivity is expected just for the presence of acceptor type defects. This is in line with Nowotny et al.'s results $[3,30]$. Regardless of the $\mathrm{Cr}$ doping level, the $\mathrm{r}-\mathrm{TiO}_{2}$ crystals reveal a clear $n$-type conductivity around $10^{2} \mathrm{~Pa}[54]$ and especially at $10^{-2} \mathrm{~Pa}$ as indicated with the red line in fig. 2.9. Here the dominant defect is $\mathrm{V}_{\mathrm{O}}$ and all crystals are in the reduced regime.

Bak et al. [53] derived defect diagrams for $\mathrm{r}-\mathrm{TiO}_{2}$ and for $\mathrm{r}-\mathrm{TiO}_{2}$ containing significant acceptor type defects, which are shown in fig. 2.10a and fig. 2.10b, respectively. The defect diagrams show the concentration of different species as a function 


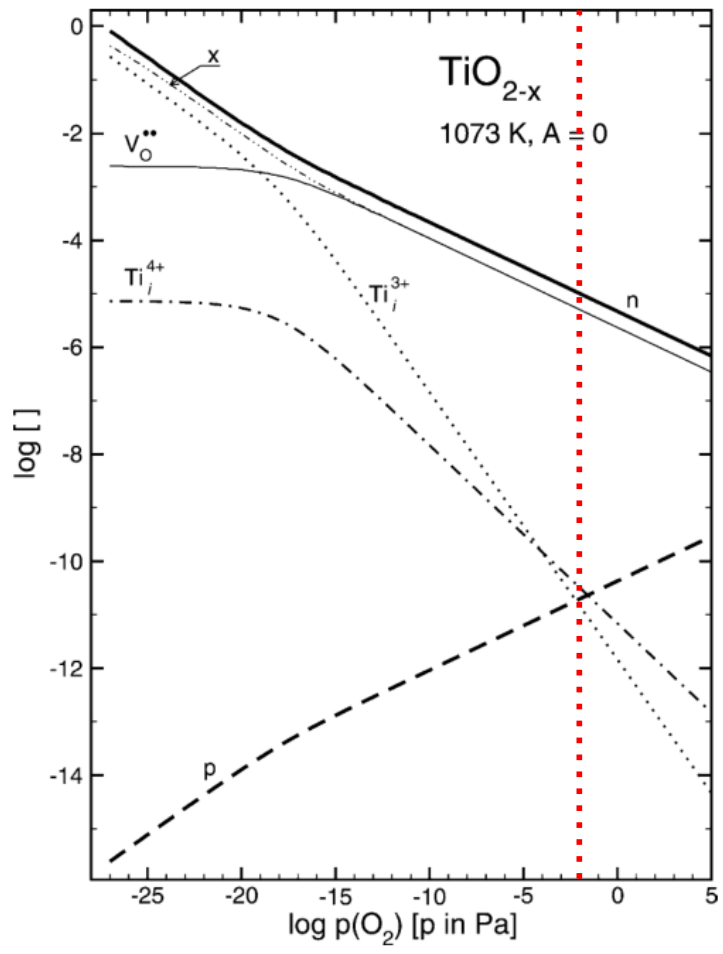

(a) No $n-p$ transition is observed, the crystal stays $n$-type for the whole pressure range.

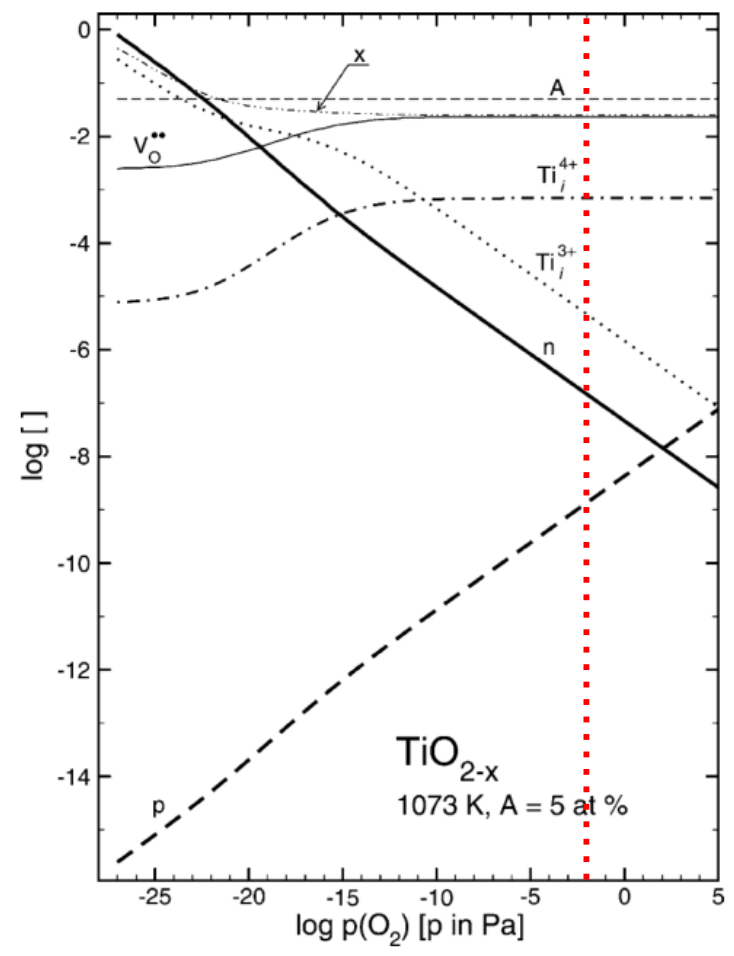

(b) $n$ - $p$ transitions observed, the acceptor defects supply the holes.

Figure 2.10.: Concentration of different intrinsic defects in (a) an undoped $(\mathrm{A}=$ $0)$ and $(\mathrm{b})$ an acceptor-doped $(\mathrm{A}=5 \mathrm{at} \%) \mathrm{r}-\mathrm{TiO}_{2}$ crystal displayed as a function of oxygen partial pressure $p\left(\mathrm{O}_{2}\right)$ at a temperature of $1073 \mathrm{~K}$. Around $10^{-2} \mathrm{~Pa}$ (red dotted line) the dominant intrinsic defect is $\mathrm{V}_{\mathrm{O}}$. The "brackets [ ] denote the concentration of defects in site ratio" [53]. Adapted with permission from Bak et al. [53]. Copyright 2003 Elsevier.

of $p\left(\mathrm{O}_{2}\right)$. These diagrams carry a lot of details which are, for simplicity, not discussed here. Both diagrams show that $\mathrm{V}_{\mathrm{O}}$ dominates the intrinsic defects in the $p\left(\mathrm{O}_{2}\right)$ range around $10^{-2} \mathrm{~Pa}$ which is indicated by the red dotted line in fig. 2.10a and 2.10b. Thus, both crystals are in the reduced regime and are of $n$-type conductivity at this oxygen activity. The influence of acceptor type defects appears mainly in the high oxygen pressure range (oxidized regime), cf. fig. 2.10b.

Nowotny et al. constructed a similar defect disorder diagram based on their measurements $[3,24,26,30,51]$, also compare fig. 2.8. The diagram shows the concentration of the intrinsic defects as well as electrons and holes as a function of $p\left(\mathrm{O}_{2}\right)$ for a given $T$ and acceptor concentration A. The corresponding defect disorder diagram is shown in fig. 2.11a. The discussion is analogous to that of fig. 2.10a, 2.11b and 2.7. Around $10^{-2} \mathrm{~Pa}$, the $\mathrm{r}^{-\mathrm{TiO}_{2}}$ crystal is of $n$-type behavior and the dominant intrinsic defect is 


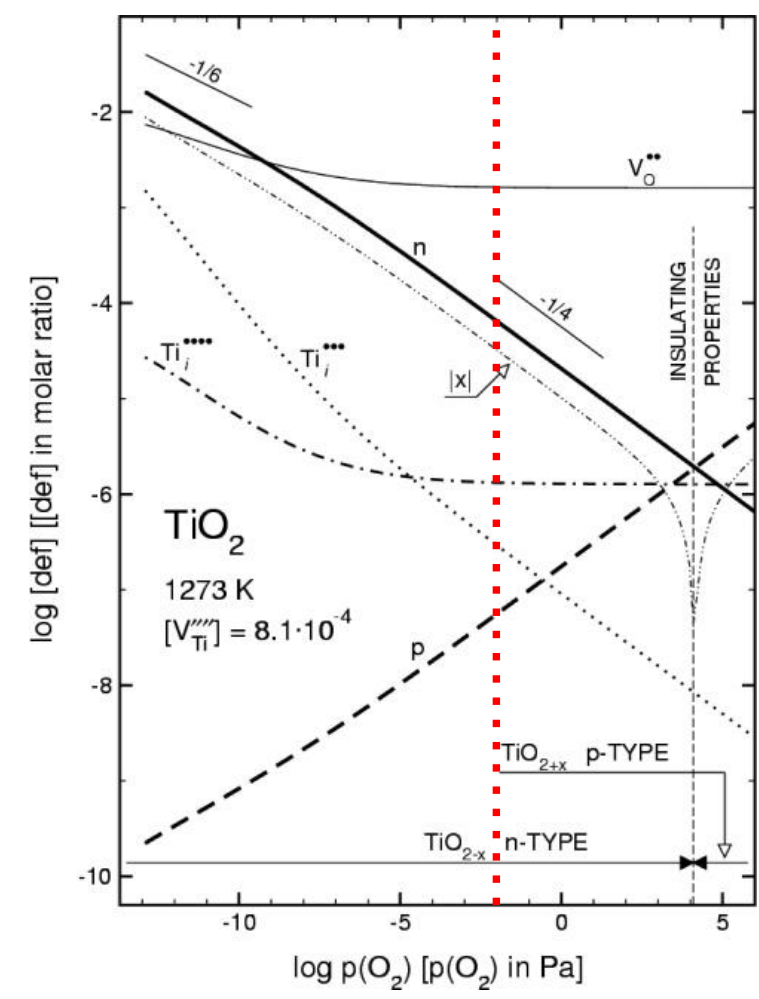

(a) Defect disorder diagram at $T=1273 \mathrm{~K}$ of $\mathrm{r}$ $\mathrm{TiO}_{2}$ [3]. The concentrations of intrinsic defects and charge carriers are shown as a function of the oxygen activity $p\left(\mathrm{O}_{2}\right)$. A fixed concentration of $\mathrm{V}_{\mathrm{Ti}}^{4-}$ as the acceptor defect was chosen. Around $10^{-2} \mathrm{~Pa}$ (red dotted line) the dominant intrinsic defect is $\mathrm{V}_{\mathrm{O}}$.

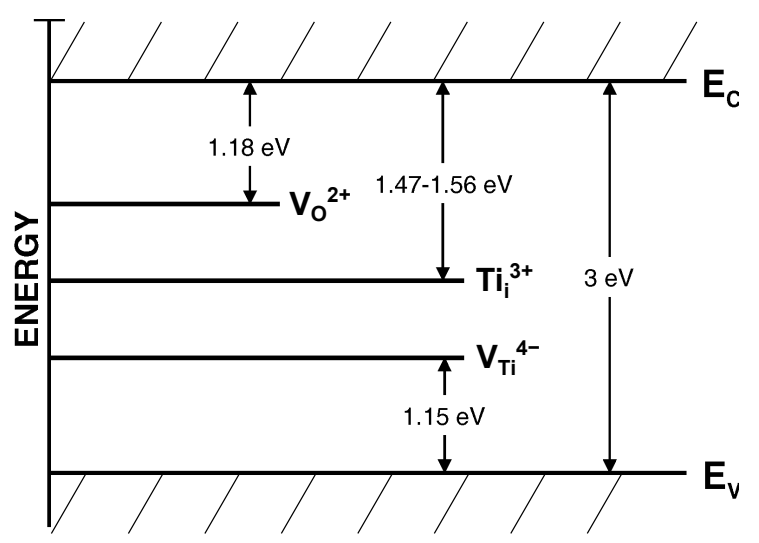

(b) Band model for the intrinsic defects of $\mathrm{r}-$ $\mathrm{TiO}_{2}$ [3]. While $\mathrm{V}_{\mathrm{O}}^{2+}$ and $\mathrm{Ti}_{\mathrm{i}}^{3+}$ are donor type defects $\mathrm{V}_{\mathrm{Ti}}^{4-}$ is a acceptor type defect in $\mathrm{r}-\mathrm{TiO}_{2}$. The band gap of approximately $3 \mathrm{eV}$ separates the conduction band minimum $\mathrm{E}_{\mathrm{C}}$ from the valence band maximum $\mathrm{E}_{\mathrm{V}}$.

Figure 2.11.: Adapted with permission from Nowotny et al. [3]. Copyright 2008 American Chemical Society.

$\mathrm{V}_{\mathrm{O}}$ as indicated in fig. 2.11a. Thus for this oxygen activity range the reduced regime is present.

Nowotny et al. [3] derived a band model from their measurement which is shown in fig. 2.11b. It shows the intrinsic defects as donor or acceptor type defects. According to fig. 2.11b $\mathrm{V}_{\mathrm{O}}^{2+}$ and $\mathrm{Ti}_{\mathrm{i}}^{3+}$ are donor type defects and $\mathrm{V}_{\mathrm{Ti}}^{4-}$ is an acceptor type defect.

As a side remark, the ionic conductivity can in general not be ignored for $\mathrm{r}-\mathrm{TiO}_{2}$ since it is a bulk property and can significantly contribute to the total conductivity especially in the vicinity of the $n-p$ transition point [30]. For details, see fig. 17 in [30].

The presented experiments from Nowotny et al. [3,24,26,30,51], from Bak et al. [32, 33,53] and from Carpentier et al. [54] were performed in an equilibrium condition at elevated temperatures. Cooling the crystal to room temperature (RT) can influence its properties [3]. The cooling rate determines if defects are in equilibrium or if a 
dynamic cooling results in a quenching of defects. The resulting conductivity can be significantly different for these cases [3]. The resulting defect disorder is difficult to predict. The general trend is a decrease of the conductivity and the corresponding defect species concentration [3].

The conductivity measurements from Nowotny et al. were evaluated in thermal equilibrium [30,51] and in a state of gas-solid equilibration $[24,26,30,51]$. This means that the chosen time scales allowed for the equilibration of $\mathrm{V}_{\mathrm{O}}$ and $\mathrm{Ti}_{\mathrm{i}}$ but not for $\mathrm{V}_{\mathrm{Ti}}$ [30]. In their experiments, the concentration of $\mathrm{V}_{\mathrm{Ti}}$ was given by the chosen conditions of the pretreatment of their $\mathrm{r}-\mathrm{TiO}_{2}$ crystal. Therefore, the $\mathrm{V}_{\mathrm{Ti}}$ concentration can be assumed to be constant within the time frame used in their experiments. Noticeable equilibration of $\mathrm{V}_{\mathrm{Ti}}$ in a mm size sample appears in time scales beyond $1000 \mathrm{~h}$ and was observed for temperatures $1123 \mathrm{~K}$ and $1323 \mathrm{~K}[25,26]$. The equilibration process of $\mathrm{V}_{\mathrm{Ti}}$ on these long time scales is called 'prolonged oxidation' [25].

The equilibration kinetics of $\mathrm{r}-\mathrm{TiO}_{2}$ at elevated temperatures were also investigated by Nowotny et al. [24] for a wide $\mathrm{O}$ pressure range. The leading question here is what are the time scales for equilibration of the $\mathrm{r}-\mathrm{TiO}_{2}$ crystal if the $\mathrm{O}$ pressure is changed. Their crystal had dimensions of approximately $2 \times 3 \times 10 \mathrm{~mm}^{3}$ [30]. The measurements were performed along the $10 \mathrm{~mm}$ axis. As stated by Nowotny et al. [24], the equilibration is controlled by the oxygen activity at the gas-solid interface. The oxygen chemical potential gradient $\left(\Delta \mu_{\mathrm{O}}\right)$ is the driving force for this equilibration process and can be determined by the surface reaction, the bulk diffusion transport, or both. Nowotny et al. [24] confirmed with their experiments that the equilibration is rate-controlled by the bulk diffusion of the defects, namely by $\mathrm{V}_{\mathrm{O}}$. Therefore, the chemical diffusion coefficient $\mathrm{D}_{\text {chem }}$ determines the equilibration kinetics. $\mathrm{D}_{\text {chem }}$ was determined as a function of $T$ and $p\left(\mathrm{O}_{2}\right)$ [24]. $\mathrm{D}_{\text {chem }}$ can be considered as the rate constant of the gas/solid equilibration kinetics [3], thus $\mathrm{D}_{\text {chem }}$ is related to the diffusion of all to the equilibration related defects. Two equilibration regimes are distinguished $[24,26]$ : in the 'operational equilibrium' which is reached in a time frame of approximately $1 \mathrm{~h}$ to $2 \mathrm{~h}$ [26], $\mathrm{V}_{\mathrm{O}}$ and $\mathrm{Ti}_{\mathrm{i}}$ are in equilibrium; in the 'effective equilibrium' which is reached in a time frame of approximately $1000 \mathrm{~h}, \mathrm{~V}_{\mathrm{Ti}}$ is also in equilibrium. The latter condition refers to prolonged oxidation $[25,26]$. $\mathrm{D}_{\text {chem }}$ is displayed against $p\left(\mathrm{O}_{2}\right)$ in fig. 2.12 according to Nowotny et al. [24].

In their results, $\mathrm{D}_{\text {chem }}$ shows a weak dependency on $p\left(\mathrm{O}_{2}\right)$ in the strongly reduced regime $\left(<10^{-5} \mathrm{~Pa}\right)$ but a significant dependency on $p\left(\mathrm{O}_{2}\right)$ in the reduced and oxidized regime [24]. A maximum of $\mathrm{D}_{\text {chem }}$ is found for the $n$ - $p$ transition point determined from their thermoelectric power measurements [51]. This behavior is explained by $\mathrm{D}_{\text {chem }}$ being proportional to $1 /(n+p)$. The dependency of $(n+p)$ on $p\left(\mathrm{O}_{2}\right)$ can be seen qualitatively in their measurements of the conductivity, cf. fig. 2.8. Interestingly, Nowotny et al. found that polycrystalline $\mathrm{r}-\mathrm{TiO}_{2}$ revealed a lower $\mathrm{D}_{\text {chem }}$ than the single crystal under the same conditions [24, fig. 10]. Thus, a "retarding" effect on oxygen 


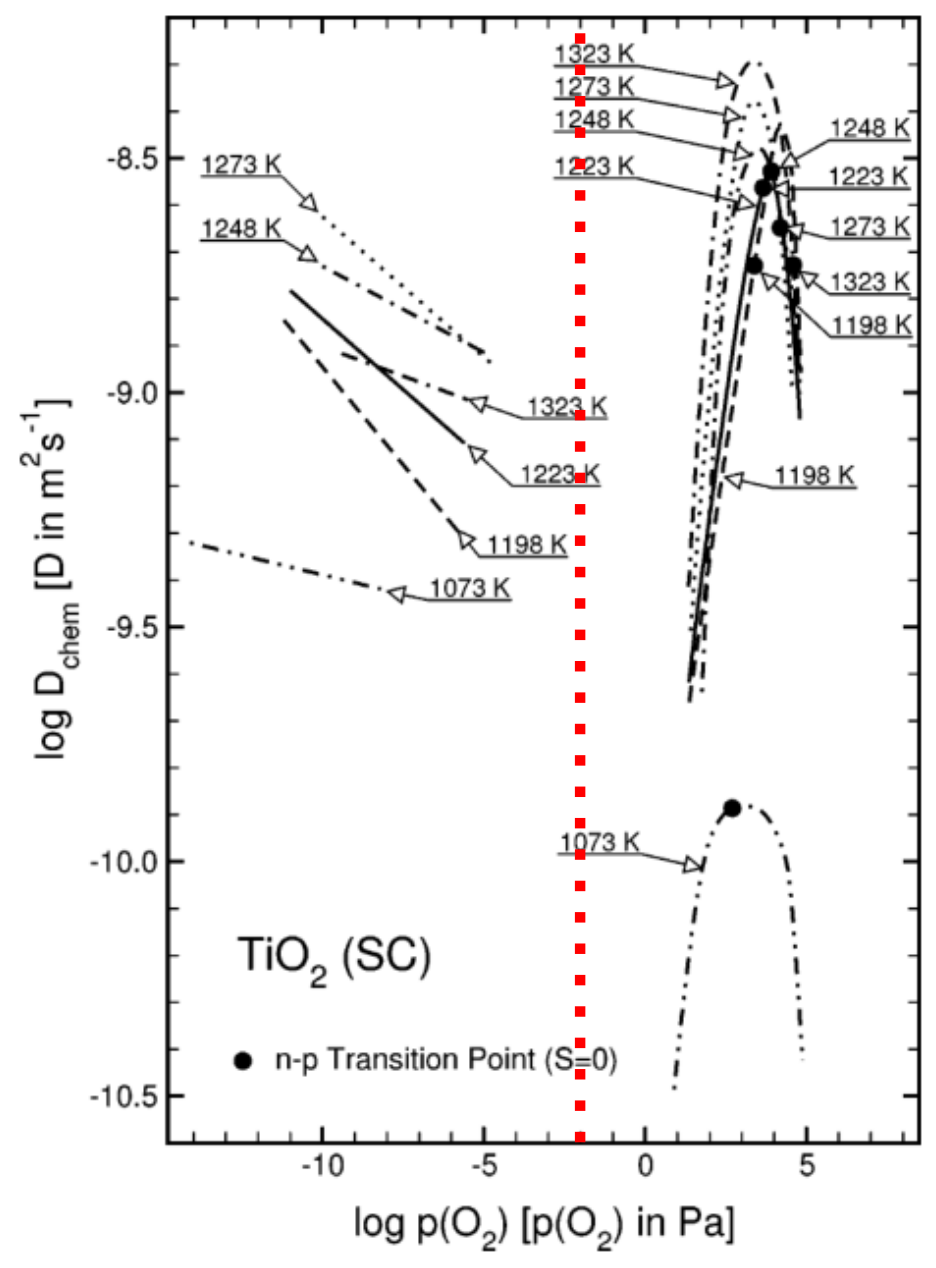

Figure 2.12.: Chemical diffusion coefficient $\mathrm{D}_{\text {chem }}$ in $\mathrm{r}-\mathrm{TiO}_{2}$ depending on the oxygen activity $p\left(\mathrm{O}_{2}\right)$ for temperature between $1073 \mathrm{~K}$ to $1323 \mathrm{~K}$. Around $10^{-2} \mathrm{~Pa}$ (red dotted line) no data are available. Adapted with permission from Nowotny et al. [24]. Copyright 2006 American Chemical Society.

diffusion is suggested for grain boundaries in polycrystalline $\mathrm{r}-\mathrm{TiO}_{2}$ [24, fig. 10]. The complex dependency of $\mathrm{D}_{\text {chem }}$ on $p\left(\mathrm{O}_{2}\right)$ is explained by Nowotny et al. [24] as being due to the relatively high deviation from stoichiometry, the interactions of the intrinsic defects, and the presence of electrons and holes alternating as the dominant charge carrier. Around $10^{-2} \mathrm{~Pa}$, no data for $\mathrm{D}_{\text {chem }}$ are available as indicated with the red dotted line in fig. 2.12.

It is important to emphasize again that a sample with a low concentration of acceptor-like defects, like $\mathrm{V}_{\mathrm{Ti}}$, does not display an oxidized regime and a $n-p$ transition, cf. fig 2.9. Hence, a decrease of $\mathrm{D}_{\text {chem }}$ as observed by Nowotny et al. [24] for high oxygen activities, cf. fig. 2.12, is not necessarily present.

Nowotny et al. studied the equilibration kinetics based on their electrical conductivity and thermoelectric power measurements [26]. At a fixed $T$, the $p\left(\mathrm{O}_{2}\right)$ was changed and the adjustment of the new equilibrium was observed by measuring the conductivity as a function of time $t$. Nowotny et al. found two kinetic regimes $[25,26]$ : in kinetic regime $\mathrm{I}$, the equilibration process is rapid and rate-controlled by the transport of $\mathrm{V}_{\mathrm{O}}$ 
as the dominant donor type defect ${ }^{7}$. In kinetic regime II, the equilibration process is slow and rate-controlled by the transport of $\mathrm{V}_{\mathrm{Ti}}$ as the dominant acceptor type defect. The equilibrium in kinetic regime I, the 'operational' equilibrium, is reached in $1 \mathrm{~h}$ to $2 \mathrm{~h}$ for $T$ between $1000 \mathrm{~K}$ and $1300 \mathrm{~K}$ [26]. The equilibrium in kinetic regime II, the 'effective' equilibrium, is reached in the order of $1000 \mathrm{~h} \mathrm{[26].} \mathrm{Nowotny} \mathrm{et} \mathrm{al.} \mathrm{see}$ the origin in a diffusion process that is 4 orders of magnitude slower than in kinetic regime I under the same conditions [26]. As an example, Nowotny et al. studied the conductivity at $1323 \mathrm{~K}$ changing $p\left(\mathrm{O}_{2}\right)$ from $26 \cdot 10^{3} \mathrm{~Pa}$ to $75 \cdot 10^{3} \mathrm{~Pa}[26]$ : in the first $0.1 \mathrm{~h}$ the conductivity changes rapidly and reaches a stable value within $0.5 \mathrm{~h}$. Thus, at those temperatures and oxygen partial pressures the major changes appear in the first minutes and are mainly completed after $30 \mathrm{~min}$. Comparable time scales apply on a similar measurement of the conductivity performed at $1123 \mathrm{~K}$. It is proposed by Nowotny et al. [26] that just the surface of the crystal is in equilibrium with all defects (including $\mathrm{V}_{\mathrm{Ti}}$ ) in kinetic regime $\mathrm{I}$. Thus, there is a gradient of $\mathrm{V}_{\mathrm{Ti}}$ from the surface to the bulk of the $\mathrm{r}-\mathrm{TiO}_{2}$ crystal. A more detailed discussion about the chemical diffusion in $\mathrm{r}-\mathrm{TiO}_{2}$ was given by Nowotny et al. [55].

Annealing times of approximately $0.5 \mathrm{~h}$ and elevated temperatures of $1023 \mathrm{~K}$ were used for the $\mathrm{r}-\mathrm{TiO}_{2}$ crystal. Compared to the established picture of the kinetic regimes from Nowotny et al. $[24,26]$, the crystals could reach operational equilibrium but not effective equilibrium. It is important to note that no data is available for $\mathrm{D}_{\text {chem }}$ around $10^{-2} \mathrm{~Pa}$, including the equilibration kinetics. Thus, the exact equilibration kinetics are unknown for this oxygen activity range.

In summary, in $\mathrm{r}-\mathrm{TiO}_{2}$ three intrinsic defects are considered important $[27,30,33]$ : $\mathrm{O}$ vacancies $\left(\mathrm{V}_{\mathrm{O}}\right)$, interstitial $\mathrm{Ti}\left(\mathrm{Ti}_{\mathrm{i}}\right)$ and $\mathrm{Ti}$ vacancies $\left(\mathrm{V}_{\mathrm{Ti}}\right)$. Which of these defects dominates the material properties, such as electrical conductivity, thermoelectric power or the chemical diffusion, depends on the temperature, oxygen activity and time scale, cf. fig. 2.7 and fig. 2.12. However, the dominant defect for all regimes, except the extremely reduced and strongly oxidized regime, is $\mathrm{V}_{\mathrm{O}}$. The $\mathrm{Ti}_{\mathrm{i}}$ concentration is insignificant in these regimes and therefore not expected to influence the results. The presence of acceptor type defects (e.g. $\mathrm{V}_{\mathrm{Ti}}$ ) allows for electron hole doping and a $p$-type conductivity at appropriately chosen $T$ and high $p\left(\mathrm{O}_{2}\right)$, as shown in fig. 2.7, 2.9 and 2.12. Although this amphoteric behavior of $\mathrm{r}-\mathrm{TiO}_{2}$ exists, $\mathrm{V}_{\mathrm{Ti}}$ is considered to not be important for this work, because of the chosen pressure range and time scales. It is important to note after the preceding discussion of this section that the properties of $\mathrm{r}-\mathrm{TiO}_{2}$ do significantly depend on its defect structure and, therefore, on its preparation condition.

A discussion of the sample properties after the pretreatment performed on the $\mathrm{r}$ -

\footnotetext{
${ }^{7}$ The influence of $\mathrm{Ti}_{\mathrm{i}}$ on the equilibration is considered as insignificant by Nowotny et al. [26] since their concentration is considerably lower than that of $\mathrm{V}_{\mathrm{O}}$ and their diffusion is comparable to that of $\mathrm{V}_{\mathrm{O}}$.
} 
$\mathrm{TiO}_{2}$ single crystals, after the thermal annealing in the sputter system and after the subsequent deposition of Pd as performed, is given in chap. 5.1, and is based on the results presented in this section.

\subsubsection{Hydrogen interactions with $\mathbf{r}-\mathrm{TiO}_{2}$}

The interaction of hydrogen with $\mathrm{r}-\mathrm{TiO}_{2}$ is an important topic since the defect chemistry of $\mathrm{r}-\mathrm{TiO}_{2}$ influences its properties significantly, cf. chap. 2.1.3. There are a lot of indications that, even at RT, hydrogen interacts with $\mathrm{r}-\mathrm{TiO}_{2}[56,57]$. Hydrogen behaves differently in $\mathrm{r}-\mathrm{TiO}_{2}$ than in other 'typical' semiconductors [57]. For example, in $\mathrm{Si}$ it saturates dangling bonds and acts as a shallow donor of the material. This section gives a short overview on the knowledge of hydrogen interaction with $\mathrm{r}-\mathrm{TiO}_{2}$.

Theoretical considerations: Filippone et al. [57] performed DFT calculations of hydrogen containing $\mathrm{r}-\mathrm{TiO}_{2}$ on the base of the local spin density-generalized gradient approximation with the Hubbard U correction (LSD-GGA+U). They simulated interstitial hydrogen $\left(\mathrm{H}_{\mathrm{i}}\right), \mathrm{V}_{\mathrm{O}}$ and substitutional hydrogen $\left(\mathrm{H}_{\mathrm{O}}\right)$. In the latter, one hydrogen occupies the $\mathrm{O}$ site in an $\mathrm{V}_{\mathrm{O}}$ and is thus sometimes referred to as a hydrogen- $\mathrm{V}_{\mathrm{O}}$ complex. All defects appear to be deep donor type defects with energy levels approximately $1.6 \mathrm{eV}$ to $1.9 \mathrm{eV}$ above the valence band maximum. $\mathrm{H}_{\mathrm{i}}$ was found to bind to an $\mathrm{O}$ site being in line with the appearance of $\mathrm{O}-\mathrm{H}$ bonds with IR spectroscopy $[4,58,59]$. The interstitial hydrogen thus exists as a $\mathrm{O}-\mathrm{H}$ complex in the $\mathrm{r}-\mathrm{TiO}_{2}$ lattice and is therefore also referred to as $\mathrm{OH}_{\mathrm{O}}$. The concentration of $\mathrm{H}_{\mathrm{i}}$ was estimated with approximately $7 \cdot 10^{16} \mathrm{~cm}^{-3}$ in the 'perfect' $\mathrm{r}-\mathrm{TiO}_{2}$ lattice at RT. It was also found by Filippone et al. that a $\mathrm{H}_{2}$ molecule is not stable in the $\mathrm{r}-\mathrm{TiO}_{2}$ lattice but always decomposes into the interstitial species. Interestingly, Filippone et al. found that the introduction of $\mathrm{V}_{\mathrm{O}}$ to $\mathrm{r}-\mathrm{TiO}_{2}$ can result in high hydrogen solubility even at RT. The reason is a reduced energy of $\mathrm{H}_{\mathrm{O}}[57]$ in comparison to the isolated defects $\left(\mathrm{H}_{\mathrm{i}}+\mathrm{V}_{\mathrm{O}}\right)$.

Bjørheim et al. [50] simulated $\mathrm{H}_{\mathrm{i}}$ and $\mathrm{H}_{\mathrm{O}}$ in undoped $\mathrm{r}-\mathrm{TiO}_{2}$ in the framework of DFT and compared the results of different functionals. Their calculations yield a clear dominance of $\mathrm{H}_{\mathrm{i}}$ in oxidized conditions in the temperature range from $293 \mathrm{~K}$ to $1293 \mathrm{~K}$. This is a reasonable finding since the concentration of $\mathrm{V}_{\mathrm{O}}$ is low in the oxidized regime, cf. chap. 2.1.3.

Experimental considerations: Different approaches exist in literature to load $\mathrm{r}-\mathrm{TiO}_{2}$ with hydrogen. According to Diebold [27], molecular hydrogen interacts just weakly with $\mathrm{r}-\mathrm{TiO}_{2}$ surfaces. Thus, a loading of $\mathrm{r}-\mathrm{TiO}_{2}$ in a hydrogen environment is not effective, at least not at RT. A first approach to overcome the weak interaction between $\mathrm{H}_{2}$ and $\mathrm{r}-\mathrm{TiO}_{2}$ is to dissociate the $\mathrm{H}_{2}$ to $\mathrm{H}$. One way to receive the dissociation is to connect an appropriate catalyst, for example $\mathrm{Pd}[60,61]$, to the $\mathrm{r}-\mathrm{TiO}_{2}$. Another way of loading $\mathrm{r}-\mathrm{TiO}_{2}$ with hydrogen is the use of an oxygen-water vapor mixture $[58,62,63]$ 
at elevated temperatures. Also, other approaches of hydrogen loading of $\mathrm{r}-\mathrm{TiO}_{2}$ do exist, for example loading in a hydrogen $[47,59]$ or hydrogen-argon mixture [52] at elevated temperatures.

The corresponding defect chemistry reactions were suggested by Erdal et al. [64] for reducing and oxidizing conditions, respectively:

$$
\begin{aligned}
\mathrm{H}_{2}(\mathrm{~g})+2 \mathrm{O}_{\mathrm{O}}^{\times} & =2 \mathrm{H}_{\mathrm{i}}^{+}+2 e^{-} \\
2 \mathrm{H}_{2} \mathrm{O}(\mathrm{g})+2 \mathrm{O}_{\mathrm{O}}^{\times} & =\mathrm{V}_{\mathrm{Ti}}^{4-}+4 \mathrm{H}_{\mathrm{i}}^{+}
\end{aligned}
$$

or it can be written for reduced $\mathrm{r}-\mathrm{TiO}_{2}$ with significant $\mathrm{V}_{\mathrm{O}}$ present as [64]:

$$
2 \mathrm{H}_{2} \mathrm{O}(\mathrm{g})+\mathrm{O}_{\mathrm{O}}^{\times}+\mathrm{V}_{\mathrm{O}}^{2+}=2 \mathrm{H}_{\mathrm{i}}^{+}
$$

the latter equation is also known as the hydration of oxygen vacancies. A reaction for the formation of a substitutional hydrogen defect is still missing in literature.

Johnson et al. [58] heated an undoped $\mathrm{r}-\mathrm{TiO}_{2}$ crystal in very dry hydrogen to $1073 \mathrm{~K}$ and analyzed it with infrared (IR) spectroscopy. In these conditions, hydrogen was found to be only weakly incorporated in $\mathrm{r}-\mathrm{TiO}_{2}$, with concentrations below $10^{17} \mathrm{~cm}^{-3}$. This finding confirms the conclusion of Diebold [27] and is in line with simulated concentrations of $\mathrm{H}_{\mathrm{i}}$ in $\mathrm{r}-\mathrm{TiO}_{2}$ [57]. After annealing $\mathrm{r}-\mathrm{TiO}_{2}$ at elevated temperatures in hydrogen, a significant amount of interstitial $\mathrm{Ti}$ was suggested to be present which is in line with the experiments and interpretation of Nowotny [52], which is discussed further below. Heating $\mathrm{r}-\mathrm{TiO}_{2}$ at the same temperature in an water-oxygen gas mixture yields to large concentrations of hydrogen in the crystal and nearly no $\mathrm{Ti}_{\mathrm{i}}$ : for a $\mathrm{Fe}$ doped $\mathrm{r}-$ $\mathrm{TiO}_{2}$ crystal, Johnson et al. [58] gained a concentration of approximately $6 \cdot 10^{19} \mathrm{~cm}^{-3}$ hydrogen atoms in a $\mathrm{r}-\mathrm{TiO}_{2}$ crystal. Doping can increase the incorporated amount of hydrogen substantially [58].

Other studies using infrared spectroscopy $[4,58,59]$ on $\mathrm{r}-\mathrm{TiO}_{2}$ crystals loaded with hydrogen revealed the presence of $\mathrm{O}-\mathrm{H}$ bonds. This suggests the possible incorporation of hydrogen in $\mathrm{r}-\mathrm{TiO}_{2}$ as an interstitial defect where hydrogen is bonded to an oxygen site. This is in line with theoretical calculations [50,57]. Herklotz et al. [59] performed IR absorption spectroscopy on initially undoped single crystals in a temperature range from $5 \mathrm{~K}$ to $110 \mathrm{~K}$. The $\mathrm{r}-\mathrm{TiO}_{2}$ was loaded in a hydrogen atmosphere of $50 \mathrm{kPa}$ at elevated temperatures $(753 \mathrm{~K}$ to $803 \mathrm{~K}$ for $12 \mathrm{~h}$ to $18 \mathrm{~h}$ ). They found the presence of two IR absorption lines for the $\mathrm{OH}$ bond which was assigned to interstitial hydrogen in a positive or neutral charge state. This is direct experimental proof of different charge states being present in hydrogenated $\mathrm{r}-\mathrm{TiO}_{2}$, and is in line with the prediction from calculations [50]. Herklotz et al. observed a strong temperature dependence of the $\mathrm{OH}$ signal in their IR measurements in this low temperature range $(5-110 \mathrm{~K})$. A further evaluation brought them to the conclusion that interstitial hydrogen has to be a shallow donor, which differs from the deep donor nature discussed above. Herklotz et al. state that most of the hydrogen egressed the $\mathrm{r}-\mathrm{TiO}_{2}$ crystal when annealed at $823 \mathrm{~K}$ in Ar. Small amounts of hydrogen were observed up to $1173 \mathrm{~K}$ in Ar. 
Hill [4] could show by combining IR absorption spectroscopy with mass loss measurements that hydrogen containing $\mathrm{r}-\mathrm{TiO}_{2}$ heated in vacuum loses water $\left(\mathrm{H}_{2} \mathrm{O}\right)$. This indicates that $\mathrm{H}_{2} \mathrm{O}$ formation is a possible mass loss channel for hydrogen containing $\mathrm{r}-\mathrm{TiO}_{2}$ at elevated temperatures and low oxygen partial pressure. This observation is in line with the defect chemistry reactions suggested by Erdal et al. [64], cf. equ 2.3 and 2.4. Hill [4] found that annealing $\mathrm{r}-\mathrm{TiO}_{2}$ crystals at elevated temperatures in hydrogen atmosphere yield to an increased conductivity of the crystals.

Wallace et al. [47] performed forward-recoil spectrometry (with helium ions) on a stoichiometric $\mathrm{r}-\mathrm{TiO}_{2}(110)$ crystal and a comparable crystal annealed at $1073 \mathrm{~K}$ in hydrogen. They found that the annealed sample contained more hydrogen than the initial $\mathrm{r}-\mathrm{TiO}_{2}$ crystal. The hydrogen reduced crystal contained approximately $4 \%$ hydrogen while the initial crystal contained approximately $1 \%$. Wallace et al. concluded from the presence of a gradient in the $\mathrm{Ti} / \mathrm{O}$ ratio present from the surface to the bulk that the limiting factor is a diffusion process of hydrogen into $\mathrm{r}-\mathrm{TiO}_{2}$. Thus, hydrogen diffuses into the bulk of $\mathrm{r}-\mathrm{TiO}_{2}$ during a hydrogen-atmosphere reduction [47] However, with the techniques used, no insights could be gained about the process or the involved atomic or molecular species.

Norby [63] derived concentrations of hydrogen related defects from literature data in $\mathrm{r}-\mathrm{TiO}_{2}$. He suggests a dominant behavior of protonated and hydrated defects, respectively, with a $\mathrm{H}_{2} \mathrm{O}$ partial pressure of $3 \mathrm{kPa}$. He mentions that at higher $\mathrm{H}_{2} \mathrm{O}$ partial pressures (above $1 \mathrm{MPa}$ and at $298 \mathrm{~K}$ ) also defect complexes between hydrogen and $\mathrm{V}_{\mathrm{Ti}}$ can be stable.

Nowotny [52] analyzed a $\mathrm{r}-\mathrm{TiO}_{2}$ single crystal in the oxidized and reduced regime based on a Jonker analysis ${ }^{8}$. The equilibrium of the reduced regime was established in a hydrogen/water vapor atmosphere diluted in Ar at elevated temperatures. Therefore, the influence of hydrogen needs to be considered as resulting in a modified lattice charge neutrality condition [52] if compared with the one from chap. 2.1.3:

$$
2\left[\mathrm{~V}_{\mathrm{O}}^{2+}\right]+3\left[\mathrm{Ti}_{\mathrm{i}}^{3+}\right]+4\left[\mathrm{Ti}_{\mathrm{i}}^{4+}\right]+\left[\mathrm{H}^{+}\right]+p=n+\mathrm{V}_{\mathrm{Ti}}^{4-}
$$

Based on a comparison of the Jonker analysis for the reduced and oxidized regime, Nowotny [52] concluded that the semiconducting properties of oxidized and reduced $\mathrm{r}-\mathrm{TiO}_{2}$ differ: the presence of hydrogen has a significant effect on the parameters of the Jonker analysis. Nowotny [52] observed a reduction of the band gap and an increase in the ratio of the electron to hole mobility $\left(\mu_{n} / \mu_{p}\right)$ in the presence of hydrogen. Despite these results, deviations from the Jonker model were found $[52,65]$ : the deviations indicate that the basis of the Jonker model, which is Maxwell-Boltzmann statistics, is not valid for hydrogen in $\mathrm{r}-\mathrm{TiO}_{2}$. Nowotny suggests [65] that the protons dissolve in the $\mathrm{r}-\mathrm{TiO}_{2}$ lattice and, therefore, strong interactions within the electron gas may be present which resemble a correlated transport behavior of the electrons. Nowotny [52] states

${ }^{8}$ The Jonker analysis requires the electrical conductivity and the thermoelectric power to be measured simultaneously in the corresponding equilibrium conditions. 
that the observed changes in the reduced regime are related to the presence of protons in the $\mathrm{r}-\mathrm{TiO}_{2}$ crystal and pointed out the possibility of the formation of $\left\{\mathrm{V}_{\mathrm{Ti}}^{4-} \cdot 4 \mathrm{OH}^{+}\right\}$ defect complexes that may lead to the changed semiconducting properties. However this could not be proved.

Nowotny [52] also applied the Jonker analysis in the reduced and oxidized regime on polycrystalline $\mathrm{r}-\mathrm{TiO}_{2}$. The polycrystalline $\mathrm{r}-\mathrm{TiO}_{2}$ exhibited just 'small' changes of the electrical conductivity in the reduced regime [52]. One interpretation of this finding can be [52] that grain boundaries in $\mathrm{r}_{-} \mathrm{TiO}_{2}$ trap hydrogen less strongly than the bulk itself.

In summary, hydrogen can be incorporated into (reduced) $\mathrm{r}-\mathrm{TiO}_{2}$ as an interstitial $\left(\mathrm{H}_{\mathrm{i}}\right)$ or as a substitutional $\left(\mathrm{H}_{\mathrm{O}}\right)$ defect. $\mathrm{H}_{\mathrm{i}}$ can also be described as a substitutional defect $\mathrm{OH}_{\mathrm{O}}$ due to the strong interaction of a hydrogen with an oxygen atom. Thus $\mathrm{H}_{\mathrm{i}}$ in $\mathrm{r}-\mathrm{TiO}_{2}$ acts substantially different than in a metal. $\mathrm{H}_{\mathrm{i}}$ and $\mathrm{H}_{\mathrm{O}}$ act as deep donors in $\mathrm{r}-\mathrm{TiO}_{2}[57]$ and are suggested to be present even at normal environmental conditions. The hydrogen source from the environment can be the partial pressure of hydrogen $\left(\approx 5 \cdot 10^{-2} \mathrm{~Pa}[66]\right)$ and the partial pressure of water $(\approx 1317 \mathrm{~Pa}[66])$ in air. This indicates a significant influence of hydrogen on (reduced) $\mathrm{r}^{-\mathrm{TiO}_{2}}$ in 'daily life'.

The presented literature overview on hydrogen-r- $\mathrm{TiO}_{2}$ interactions establishes a qualitative picture of possible defect formation mechanisms $\left(\mathrm{V}_{\mathrm{O}}, \mathrm{H}_{\mathrm{i}}\right.$ and $\left.\mathrm{H}_{\mathrm{O}}\right)$. The calculations of Bjørheim et al. [50] suggest a lower energy of $\mathrm{H}_{\mathrm{O}}$ if compared to the individual defects $\left(\mathrm{V}_{\mathrm{O}}+\mathrm{H}_{\mathrm{i}}\right)$. A reduced $\mathrm{r}-\mathrm{TiO}_{2}$ crystal may supply $\mathrm{V}_{\mathrm{O}}$ and, thus, can allow for high $\mathrm{H}_{\mathrm{O}}$ concentrations in hydrogen atmosphere. However, a clear conclusion of which concentrations of these defects are present in reduced $\mathrm{r}-\mathrm{TiO}_{2}$ exposed to a hydrogen atmosphere cannot be drawn.

\subsubsection{Hydrogen diffusion in $\mathrm{r}-\mathrm{TiO}_{2}$}

A comprehensive study - for example performed for intrinsic defects in undoped $\mathrm{r}-\mathrm{TiO}_{2}$ by Nowotny et al. $[24,26,30,51]$ - of hydrogen diffusion in $\mathrm{r}^{-\mathrm{TiO}_{2}}$ single crystals has not been performed. As Herklotz et al. [59] state: the transport in $\mathrm{r}-\mathrm{TiO}_{2}$ is poorly understood. A comprehensive study is needed to investigate the oxygen activity as well as the hydrogen activity in a large activity range including the study of the equilibration kinetics. In the field of hydrogen diffusion in $\mathrm{r}-\mathrm{TiO}_{2}$, this is a task for the future. However, diffusion measurements on hydrogen in $\mathrm{r}-\mathrm{TiO}_{2}$ have been performed for a few fixed oxygen activities and are discussed further below. Besides the self diffusion, a trend was found for the hydrogen diffusivity in $\mathrm{r}-\mathrm{TiO}_{2}$ : it increases significantly with electron irradiation [67] or with illumination by resonant infrared light $[68,69]$. The first finding could be important for this work since the $\mathrm{Pd} / \mathrm{r}-\mathrm{TiO}_{2}$ samples were investigated with TEM.

Chen et al. [67] performed radiation- and electric-field-induced diffusion (REID), using a $2.0 \mathrm{MeV}$ Van de Graaff generator, on $\mathrm{r}-\mathrm{TiO}_{2}$ and found a high mobility of 
deuterium close to RT: with REID, the deuterium concentration decreased as rapidly at $340 \mathrm{~K}$ as it did without REID at $1000 \mathrm{~K}$. This hints to an enhanced mobility of hydrogen in the presence of the electron beam. Transferring this observation to a TEM also suggests an enhanced mobility of hydrogen in the presence of the electron beam. However, a quantitative comparison cannot be drawn from Chen et al.'s measurements [67].

A mechanism for the diffusion of interstitial hydrogen in $\mathrm{r}-\mathrm{TiO}_{2}$ was proposed by Bates er al. [70]. They propose that the diffusion parallel to the $c$-axis happens via jumps of hydrogen from the interstitial position to the adjacent $\mathrm{O}$ atom as depicted in the following :

$$
\mathrm{H}_{\mathrm{i}} \cdots \mathrm{O} \rightarrow \mathrm{O} \cdots \mathrm{H} \cdots \mathrm{O} \rightarrow \mathrm{O} \cdots \mathrm{H}_{\mathrm{i}}
$$

Here, ' $\cdots \mathrm{O}$ ' indicates the adjacent $\mathrm{O}$ atom along the $c$ axis. To visualize this diffusion process, $\mathrm{H}_{\mathrm{i}}$ in $\mathrm{r}-\mathrm{TiO}_{2}$ is depicted in fig. 2.13.

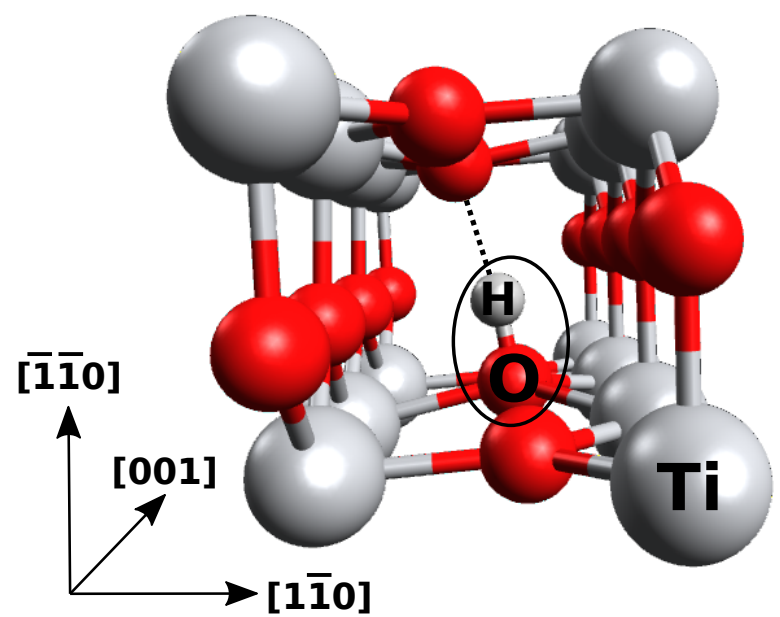

Figure 2.13.: Illustration of $\mathrm{H}_{\mathrm{i}}$ in $\mathrm{r}-$ $\mathrm{TiO}_{2}$. Here, hydrogen has a bond to an oxygen site ( $\mathrm{OH}$ bond) which is indicated with the ellipse. Additionally the hydrogen atom possesses a hydrogen bond to the opposing oxygen atom which is indicated with the black dashed lines. The shown situation can be depicted as the initial and the final situation of a diffusion process in $<001>$ direction, cf. eq. 2.5. With kind approval of M. Sotoudeh.

$\mathrm{A} \mathrm{H}_{\mathrm{i}}$ defect shares an $\mathrm{OH}$ bond between the involved $\mathrm{O}$ and $\mathrm{H}$ atom (ellipse). The $\mathrm{H}$ atom also shares a hydrogen bond (dashed line) with the opposing $\mathrm{O}$ atom in the [1̄10] direction. Following the diffusion process proposed by Bates et al. [70], hydrogen gets protonated, cf. eq. 2.5. As a proton, hydrogen can diffuse by 'hopping' in the $<001>$ direction (or along the $c$-channel) until it binds again to an $\mathrm{O}$ site and reestablishes an $\mathrm{H}_{\mathrm{i}}$ defect, cf. eq. 2.5.

The diffusion perpendicular to the $c$-axis is suggested by Bates et al. [70] by a rotation of the $\mathrm{OH}$ bond at which hydrogen is transferred to the adjacent channel, cf. $<110>$ directions in fig. 2.13 .

Johnson et al. [62] studied the diffusion of hydrogen and deuterium in $\mathrm{r}-\mathrm{TiO}_{2}$ single crystals. The $\mathrm{r}-\mathrm{TiO}_{2}$ crystals were prepared for the diffusion experiment by thermal annealing at $1123 \mathrm{~K}$ for $24 \mathrm{~h}$ in a mixture of $13.3 \mathrm{kPa} \mathrm{O}$ and $2.67 \mathrm{kPa}$ of $\mathrm{H}_{2} \mathrm{O}$. With respect to the oxygen activity regimes, as discussed in chap. 2.1.3 by Nowotny et

\footnotetext{
${ }^{9}$ Charges are neglected for simplicity.
} 
al. [30], this corresponds to the oxidized or to the poorly reduced regime. Johnson et al. note that hydrogen in $\mathrm{r}-\mathrm{TiO}_{2}$ is a possible candidate for tunneling diffusion, especially in the shorter $c$-axis direction. They used an isotope exchange technique (hydrogendeuterium) in combination with IR spectroscopy and compared their findings to ionic conductivity measurements in order to determine the temperature dependent hydrogen diffusion coefficient. The diffusion measurements of Johnson et al. [62] were performed in a temperature range from $623 \mathrm{~K}$ to $973 \mathrm{~K}$. They conducted the measurements on crystals oriented parallel and perpendicular to the $c$-axis to take the $\mathrm{r}^{-\mathrm{TiO}_{2}}$ crystal anisotropy into account. All tested $\mathrm{r}-\mathrm{TiO}_{2}$ crystals contained a significant amount of aluminum [62]. As stated by Nowotny et al. [30] impurities can influence the properties of $\mathrm{r}-\mathrm{TiO}_{2}$, such as the chemical diffusion or electrical conductivity, significantly. As noted by Johnson et al. the diffusion of hydrogen in $\mathrm{r}^{-\mathrm{TiO}_{2}}$ can be significantly smaller in the presence of $\mathrm{Ti}_{\mathrm{i}}$ or $\mathrm{V}_{\mathrm{O}}$, which indicates an attractive interaction of hydrogen with those defects. Johnson et al. [62] calculated the following diffusion coefficients parallel $D_{\| c}$ and orthogonal $D_{\perp c}$ to the $c$-axis which results in the following values at RT $(293 \mathrm{~K})$ :

$$
\begin{aligned}
D_{\| c}=1.8 \cdot 10^{-3} \exp (-0.59 \mathrm{eV} / k T) \mathrm{cm}^{2} / \mathrm{s} & =1.3 \cdot 10^{-13} \mathrm{~cm}^{2} /\left.\mathrm{s}\right|_{T=293 \mathrm{~K}} \\
& =1.3 \cdot 10^{-17} \mathrm{~m}^{2} /\left.\mathrm{s}\right|_{T=293 \mathrm{~K}} \\
D_{\perp c}=3.8 \cdot 10^{-1} \exp (-1.28 \mathrm{eV} / k T) \mathrm{cm}^{2} / \mathrm{s} & =3.8 \cdot 10^{-23} \mathrm{~cm}^{2} /\left.\mathrm{s}\right|_{T=293 \mathrm{~K}} \\
& =3.8 \cdot 10^{-27} \mathrm{~m}^{2} /\left.\mathrm{s}\right|_{T=293 \mathrm{~K}}
\end{aligned}
$$

The anisotropy of the diffusion is obvious. The diffusion perpendicular to the $c$-axis is far slower than that along the $c$-axis. No significant influence of tunneling was observed [62], and Johnson et al. [62] mentioned that their measured diffusion coefficient is lower than the one expected for "bare" tunneling. This is an important point because it clarifies that tunneling of hydrogen in $\mathrm{r}-\mathrm{TiO}_{2}$ is different from tunneling of hydrogen in a metal, such as $\mathrm{Pd}$. To the knowledge of the author, there are no comprehensive studies about the nature of hydrogen tunneling in $\mathrm{r}-\mathrm{TiO}_{2}$. Johnson et al. [62] did supply a qualitative argument for this difference in hydrogen tunneling nature: a point charge induced by hydrogen yields the formation of a polarization cloud in $\mathrm{r}-\mathrm{TiO}_{2}$. This cloud would need to follow the tunneling, which is unlikely. For tunneling, the whole defect construct would need to tunnel which seems unlikely and thus confirms Johnson et al.'s argumentation [62].

The directional diffusion in $\mathrm{r}-\mathrm{TiO}_{2}$ can be calculated after Sorensen [21, p. 160] with the following equation:

$$
D(\psi)=D_{\| c} \cos ^{2}(\psi)+D_{\perp c} \sin ^{2}(\psi)
$$

with $\psi$ the angle between the diffusion direction and the $c$ axis. In this work, $\mathrm{Pd}$ was deposited on $\mathrm{r}-\mathrm{TiO}_{2}(110)$ crystals. The hydrogen diffusion in $<110>$ direction $(\triangleq$ 
$\left.\psi=90^{\circ}\right)$ is therefore important for this work. The cos term in eq. 2.8 is therefore 0 and the sin term is 1. Equ. 2.8 simplifies, for diffusion in $<110>$ direction, to:

$$
D_{<110>}=D\left(\psi=90^{\circ}\right)=D_{\perp c}
$$

This results in a slow diffusion, cf. eq. 2.7, from the $\mathrm{Pd} / \mathrm{r}-\mathrm{TiO}_{2}$ interface to the bulk of $\mathrm{r}-\mathrm{TiO}_{2}$. In comparison, the diffusion along the interface in $<001>$ directions is fast since

$$
D_{<001>}=D\left(\psi=0^{\circ}\right)=D_{\| c}
$$

cf. eq. 2.6.

With the diffusion coefficient at RT, the order of magnitude of the time that hydrogen needs to diffuse $100 \mathrm{~nm}$ in the corresponding direction can be estimated:

$$
\begin{aligned}
& t_{\| c}=\frac{<x>^{2}}{D_{\|}} \approx 773 \mathrm{~s} \\
& t_{\perp c}=\frac{<x>^{2}}{D_{\perp}} \approx 1.3 \cdot 10^{12} \mathrm{~s} \approx 4.2 \cdot 10^{4} \text { years }
\end{aligned}
$$

Clearly $\mathrm{r}-\mathrm{TiO}_{2}$ perpendicular to the $c$-axis acts like a blocking layer for hydrogen diffusion with the conditions chosen by Johnson et al. [62].

Nandasiri et al. [71] implanted hydrogen into $\mathrm{r}-\mathrm{TiO}_{2}(110)$. They measured depth profiles $(\leq 800 \mathrm{~nm})$ of hydrogen with nuclear reaction analysis (NRA) as a function of a 30 min annealing at different temperatures in vacuum $\left(\approx 10^{-5} \mathrm{~Pa}\right)$. Nandasiri et al. discovered at $373 \mathrm{~K}$ the hydrogen depth profile broadens without changing the total hydrogen amount in the sample. At $473 \mathrm{~K}$ a considerable amount of hydrogen was lost, and at $523 \mathrm{~K}$ no hydrogen could be detected any more with NRA. As stated by Nandasiri et al., the diffusion in $\mathrm{r}-\mathrm{TiO}_{2}$ in $<110>$ direction has not yet been addressed directly. Their measurements suggest sufficient mobility in the $\langle 110\rangle$ direction at least at $373 \mathrm{~K}$. The NRA measurements were taken at $300 \mathrm{~K}$ at which no changes were observed in the timescale of the measurement. Additionally Nandasiri et al. performed UPS and XPS measurements on the different loading states. Based on this studies they concluded that most of the hydrogen leaves the sample as $\mathrm{H}_{2}$ but some also as $\mathrm{H}_{2} \mathrm{O}$. The latter species was held responsible for the observed surface depletion of oxygen resulting in the formation of $\mathrm{Ti}_{\mathrm{i}}$ on the surface.

In summary, the diffusion in $\mathrm{r}-\mathrm{TiO}_{2}$ single crystals is anisotropic. Along the crystal's $c$-axis the diffusion is significantly faster than orthogonal to it, cf. eq. 2.6 and 2.7. Thus, for macroscopic samples, a diffusion barrier can exist along the $\mathrm{r}-\mathrm{TiO}_{2}<110>$ directions. The produced $\mathrm{Pd} / \mathrm{r}-\mathrm{TiO}_{2}$ samples exhibit $<110>$ and $<001>$ directions along the interface and $\mathrm{a}<110>$ direction perpendicular to it (into the bulk of the $\mathrm{r}-\mathrm{TiO}_{2}$ crystals). Hence, diffusion parallel to the interface is fast when compared to the diffusion into the $\mathrm{r}-\mathrm{TiO}_{2}$ bulk. 
As was shown by Nowotny et al. [24] for the oxygen activity and the corresponding equilibration of the intrinsic defects, the actual hydrogen diffusion coefficient can strongly depend on the defect equilibration in the sample.

\subsection{Palladium (Pd)}

This chapter presents the properties of $\mathrm{Pd}$ and the hydrogen-Pd (H-Pd) system in the applied hydrogen pressure range.

$\mathrm{Pd}$ is a metal with a fcc crystal structure. Important bulk properties for this work are summarized in tab. 2.3.

\begin{tabular}{ll}
\hline \hline Palladium & \\
\hline crystal system & face-centered cubic (fcc) [72] \\
crystal class & hexakisoctahedral [15] \\
(/point group) & $\mathrm{F} 4 / \mathrm{m} \overline{3} 2 / \mathrm{m}[15,73]$ (Hermann-Mauguin notation) \\
& $\mathrm{O}_{\mathrm{h}}[15]$ (Schoenflies notation) \\
space group & $\mathrm{Fm} \overline{3} \mathrm{~m}($ no. 225) [15,73] \\
lattice parameter & $a=(0.38902 \pm 0.00006) \mathrm{nm}[72]$ \\
density & $(12007 \pm 6) \mathrm{kg} / \mathrm{m}^{-3}[72]$ \\
work function & $(4.99 \pm 0.04) \mathrm{eV}[74]$ \\
melting point & $(1828.0 \pm 0.1) \mathrm{K}[72]$ \\
hydrogen diffusion & $(2.94 \pm 0.2) \cdot \exp \left(-\frac{(0.2282 \pm 0.0022) \mathrm{eV}}{k_{\mathrm{B}} T} \mathrm{~cm}^{2} / \mathrm{s}[75]\right.$ \\
coefficient & \\
\hline \hline
\end{tabular}

Table 2.3.: Properties of bulk palladium.

The following quantities are important for the X-ray measurements described later on in chap. 3.5:

The ideal $\operatorname{Pd}(111)$ lattice plane distance is $d_{111}=(2.2460 \pm 0.0004) \AA$ as derived from the $\mathrm{Pd}$ lattice parameter $a$, cf. tab. 2.3. The mass attenuation coefficient for $\mathrm{Cu} K_{\alpha}$ radiation $(\sim 8 \mathrm{keV}[76])$ is calculated with $\frac{\mu}{\rho} \simeq 201.7 \mathrm{~cm}^{2} / \mathrm{g}$ from Hubbell and Seltzer [77]. With the density of $\mathrm{Pd} \rho$, cf. tab. 2.3, it follows for the attenuation coefficient $\mu: \mu=\frac{\mu}{\rho} \cdot \rho=(242181.19 \pm 121.02) 1 / \mathrm{m}$. The extinction coefficient $\beta$ can be approximated by $\beta=\frac{\lambda \mu}{4 \pi}=(2.9691 \pm 0.0015) \cdot 10^{-6}[78$, slide 7$]$.

Molecular hydrogen is split on Pd surfaces to atomic hydrogen [60,61]. The atomic hydrogen is solvable in the Pd lattice where it occupies interstitial octahedral sites [80]. H-Pd isotherms for different temperatures were summarized by Manchester et al. [79] and are shown in fig. 2.14. Here, the (logarithmic) hydrogen pressure $p$ is given as a function of the atomic $\mathrm{H} / \mathrm{Pd}$ ratio $c_{\mathrm{H}}$ for different temperatures. According to fig. 2.14 for low hydrogen pressures (left side) the $\mathrm{H} / \mathrm{Pd}$ ratio is small for all temperatures $(\alpha$ phase). Increasing the pressure eventually leads to the appearance of a plateau with 


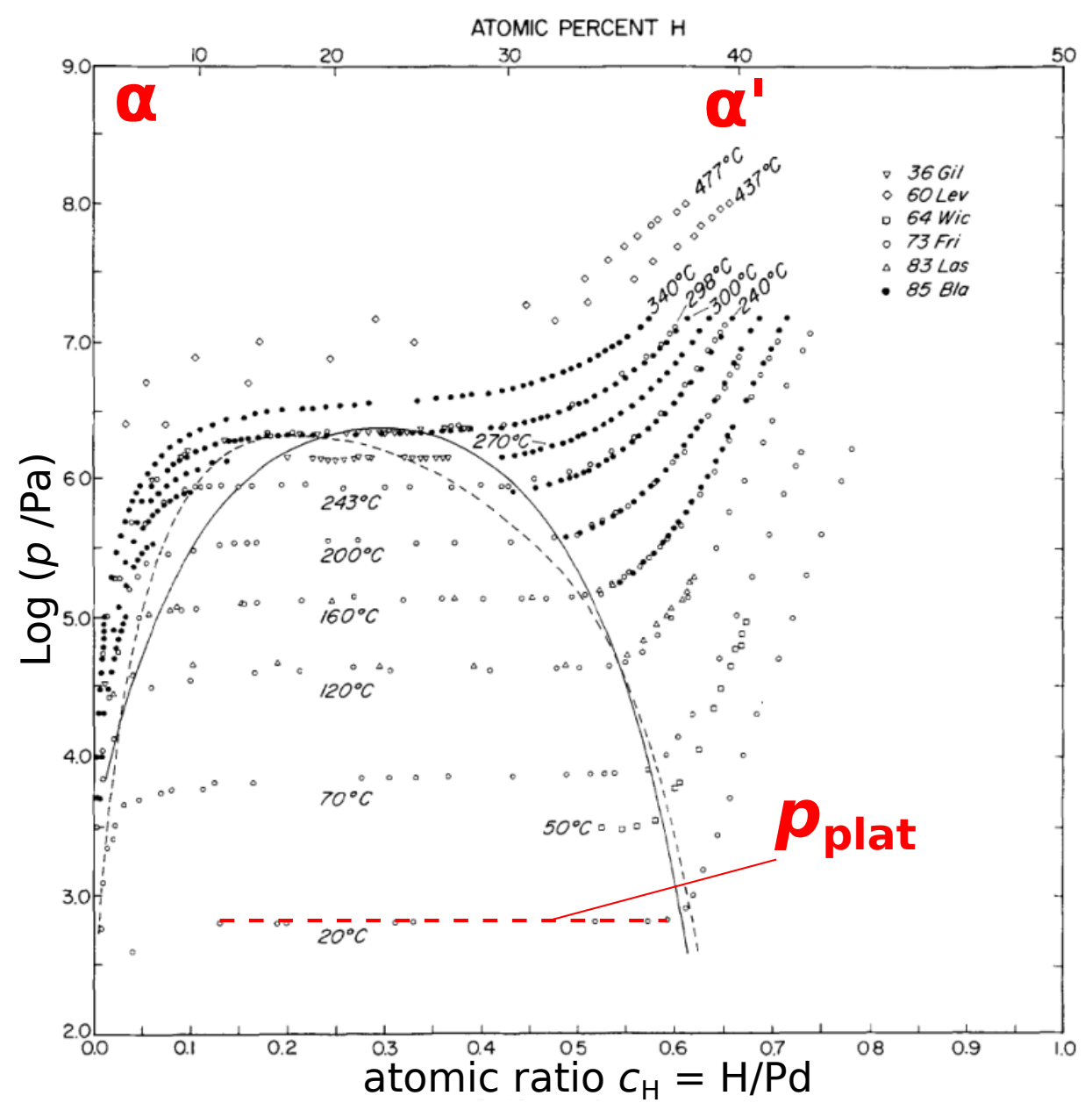

Figure 2.14.: Isotherms of the hydrogen pressure $p$ as a function of the atomic $\mathrm{H} / \mathrm{Pd}$ ratio $c_{\mathrm{H}}$ are shown for different temperatures. The $\alpha$ phase of $\mathrm{H}-\mathrm{Pd}$ solves small amounts of hydrogen up to the plateau pressure $p_{\text {plat }}(T)$ at which the H-Pd system phase separates in the $\alpha$ and $\alpha^{\prime}$ phase. $p_{\text {plat }}$ is indicated with the red dashed line for the $20^{\circ} \mathrm{C}(=293 \mathrm{~K})$ isotherm. Increasing the pressure above the plateau pressure brings the H-Pd system in the $\alpha^{\prime}$ phase that can solve high amounts of hydrogen. Adapted with permission from Manchester et al. [79]. Copyright 1994 Springer Nature.

plateau pressure $p_{\text {plat }}(T)$ for all temperatures, cf. fig 2.14. The H-Pd system phase separates into an $\alpha$ and a hydride phase ( $\alpha^{\prime}$ phase). Increasing the pressure above the plateau pressure transforms the H-Pd system completely to the $\alpha^{\prime}$ phase (right side) which can solve high amounts of hydrogen per $\mathrm{Pd}$ and thus greatly increases the $\mathrm{H} / \mathrm{Pd}$ ratio.

At RT (293 K), the $\mathrm{Pd}-\mathrm{H}$ plateau pressure $p_{\text {plat }}$ is approximately $620 \mathrm{~Pa}$ [80, eq. 3.9], cf. red dashed line in fig. 2.14, and a maximum of approximately $0.008 \mathrm{H} / \mathrm{Pd}[80$, tab. 3.1] can be solved in the alpha phase. Relevant for the EELS experiments are 
hydrogen pressures up to $10 \mathrm{~Pa}$. At $10 \mathrm{~Pa}$, an atomic ratio of approximately $c_{\mathrm{H}}=$ $9 \cdot 10^{-4} \mathrm{H} / \mathrm{Pd}$ results at RT [80, eq. 3.4]. Hence, the stored amount of hydrogen, at up to $10 \mathrm{~Pa}$, is small. Assuming a $\mathrm{Pd}(111)$ film adhered on a rigid substrate allows the calculation of the normal (out-of-plane) strain $\epsilon_{0}$ that is associated with $c_{\mathrm{H}}$. Applying the model of linear elasticity to this situation, as for example shown by Wagner [81], the normal strain calculates as [81, eq. 2.41]:

$$
\epsilon_{0}=0.126 \cdot c_{\mathrm{H}}
$$

Thus, at $10 \mathrm{~Pa}$ and RT this yields to:

$$
\epsilon_{0}=0.126 \cdot 9 \cdot 10^{-4} \approx 1.1 \cdot 10^{-4}=1.1 \cdot 10^{-2} \%
$$

The corresponding lateral (in-plane) stress $\sigma$ results with approximately $|-31| \mathrm{MPa}[81$, eq. 2.34b]. The hydrogen induced expansion and the corresponding lateral stress, hence, are small. Thus, no relevant expansion is expected for $\operatorname{Pd}(111)$ films at RT up to a pressure of $10 \mathrm{~Pa}$ and can therefore be neglected for the in situ EELS measurements.

In contrast to $10 \mathrm{~Pa}$, an applied hydrogen pressure of $500 \mathrm{~Pa}$ is close to $p_{\text {plat }} \approx 620 \mathrm{~Pa}$ of bulk Pd. At $500 \mathrm{~Pa}$, an atomic ratio of approximately $c_{\mathrm{H}}=6 \cdot 10^{-3} \mathrm{H} / \mathrm{Pd}$ results at RT. The hydrogen induced expansion of a $\operatorname{Pd}(111)$ film in normal direction is therefore [81, eq. 2.41]:

$$
\epsilon_{0}=0.126 \cdot 6 \cdot 10^{-3} \approx 7.6 \cdot 10^{-4}=7.6 \cdot 10^{-2} \%
$$

The corresponding lateral (in-plane) stress is $\sigma=|-217| \mathrm{MPa}$ [81, eq. 2.34b]. This value is significantly higher than the $10 \mathrm{~Pa}$ case. This work investigates whether defects in $\mathrm{Pd}$ are generated in this hydrogen pressure range.

The formed atomic hydrogen is transported rapidly in $\mathrm{Pd}[75,82]$. At RT the diffusion coefficient is approximately $3.5 \cdot 10^{-11} \mathrm{~m}^{2} \mathrm{~s}^{-1}[75]$, this results in $\mu \mathrm{m}$ diffusion length in under a second. Thus, changing the hydrogen partial pressure and the hydrogen chemical potential, respectively, results in a very fast equilibration of the H-Pd system.

In summary, $\mathrm{Pd}$ can be considered mainly as an atomic hydrogen source for the $\mathrm{Pd} / \mathrm{r}-\mathrm{TiO}_{2}$ system in the investigated hydrogen pressure range up to $10 \mathrm{~Pa}$. Hydrogen diffuses fast in $\mathrm{Pd}$, the H-Pd system thus adapts quickly to changes in the hydrogen pressure and can be assumed to be in equilibrium with the hydrogen environment for all performed experiments. With hydrogen pressures up to $500 \mathrm{~Pa}$, defects can be created in the Pd which was investigated.

\subsection{Palladium/rutile titanium dioxide $\left(\mathrm{Pd} / \mathrm{r}-\mathrm{TiO}_{2}\right)$}

In chap. 2.1 and 2.2 both $\mathrm{r}-\mathrm{TiO}_{2}$ and $\mathrm{Pd}$ are discussed individually. For this work the hydrogen interaction with the combined $\mathrm{Pd} / \mathrm{r}-\mathrm{TiO}_{2}$ system was of interest. This 
chapter presents the mechanical and electrical properties that accompany the formation of the $\mathrm{Pd} / \mathrm{r}-\mathrm{TiO}_{2}$ interface in chap. 2.3.1 and chap. 2.3.2. An overview of the established knowledge of the hydrogen interaction with the $\mathrm{Pd} / \mathrm{r}-\mathrm{TiO}_{2}$ system is given in chap. 2.3.2.1.

\subsubsection{Growth and expected lattice misfit of $\mathrm{Pd}$ on $\mathbf{r}-\mathrm{TiO}_{2}$}

Thin films on rigid substrates can exhibit high lateral (or in-plane) stresses. The lateral stress originates from a lattice misfit between the crystal planes of the film and those of the underlying substrate. In the following, the stresses and strains are calculated that are expected for $\mathrm{Pd} / \mathrm{r}-\mathrm{TiO}_{2}$ samples.

$\mathrm{Pd}$ is expected to grow by the Volmer-Weber growth mode with a (111) $\|(110) \mathrm{r}-\mathrm{TiO}_{2}$ orientation relationship in the normal (or out-of-plane) direction [27]. For simplicity, the following orientation relationship is assumed in lateral film direction: $\operatorname{Pd}[0 \overline{1} 1] \| \mathrm{r}-$ $\mathrm{TiO}_{2}[001]$ and $\mathrm{Pd}[\overline{2} 11] \| \mathrm{r}-\mathrm{TiO}_{2}[\overline{1} 10]$, respectively. The knowledge of this orientation relationship allows an investigation into the lattice misfit and the corresponding stresses in both lateral film directions. The anisotropy of the $\mathrm{r}-\mathrm{TiO}_{2}(110)$ surface suggests these properties are anisotropic. The anisotropy is expected due to the two different $\mathrm{r}^{-\mathrm{TiO}_{2}}$ lattice plane distances that appear on the (110) surface, namely $d_{001}$ and $d_{\overline{1} 10}$. Besides the pure lattice misfit (at RT), an additional thermal stress and strain are considered. The thermal contribution is calculated for cooling from $1023 \mathrm{~K}$ to RT $(293 \mathrm{~K})$ using the temperature dependent lattice constants of $\mathrm{Pd}$ and $\mathrm{r}-\mathrm{TiO}_{2}$. The additional thermal stress is anisotropic and tensile in nature, as presented in details in chap. 2.3.1.1. It is important to state that in the following consideration the $\mathrm{r}-\mathrm{TiO}_{2}$ is assumed to be rigid and the $\mathrm{Pd}$ lattice has to adopt to the $\mathrm{r}-\mathrm{TiO}_{2}$ surface.

At $1023 \mathrm{~K}$, the lattice parameter of $\mathrm{Pd}$ is $a=3.9300 \AA$ [72] and those of $\mathrm{r}-\mathrm{TiO}_{2}$ are $a=4.6101 \AA$ and $c=2.9753 \AA$ [83]. With the lattice parameters, the following lattice plane distances occur: $d_{\overline{2} 11}^{\mathrm{Pd}}=1.6044 \AA, d_{\overline{1} 10}^{\mathrm{r}-\mathrm{TiO}_{2}}=3.2599 \AA, d_{0 \overline{1} 1}^{\mathrm{Pd}}=2.7789 \AA$ and $d_{001}^{\mathrm{r}-\mathrm{TiO}_{2}}=2.9753 \AA$. These lattice plane distances are summarized, together with corresponding RT values in tab. 2.4. Considering these $d$ values, the minimal lattice misfit $\Delta d$ is obtained for fifteen $\mathrm{Pd}(0 \overline{1} 1)$ planes parallel to fourteen $\mathrm{r}-\mathrm{TiO}_{2}(001)$ planes and two $\operatorname{Pd}(\overline{2} 11)$ planes parallel to one $\mathrm{r}-\mathrm{TiO}_{2}(\overline{1} 10)$ plane $^{10}$ :

$$
\Delta d\left[\operatorname{Pd}(\overline{2} 11) \| \mathrm{r}-\mathrm{TiO}_{2}(\overline{1} 10)\right]=1 \cdot 3.2599 \AA-2 \cdot 1.6044 \AA \approx 0.0510 \AA
$$

and

$$
\Delta d\left[\operatorname{Pd}(0 \overline{1} 1) \| \mathrm{r}-\mathrm{TiO}_{2}(001)\right]=14 \cdot 2.9753 \AA-15 \cdot 2.7789 \AA \approx-0.0301 \AA
$$

Considering $\Delta d$ as the length that the Pd lattice has to change for a matching of the atomic planes the corresponding stress $\sigma$ and strain $\epsilon$ can be calculated as:

\footnotetext{
${ }^{10}$ Of course the lattice misfit is even higher if all $\mathrm{Pd}$ planes are assumed to stack each on the corresponding $\mathrm{r}-\mathrm{TiO}_{2}$ plane in lateral direction.
} 


$$
\begin{aligned}
\sigma\left[\operatorname{Pd}(h k l) \| \mathrm{r}-\mathrm{TiO}_{2}(u v w)\right]= & \frac{E_{h k l}^{\mathrm{Pd}}}{1-\nu^{2}} \cdot\left\{\epsilon\left[\operatorname{Pd}(h k l) \| \mathrm{r}-\mathrm{TiO}_{2}(u v w)\right]\right. \\
& \left.+\nu \cdot \epsilon\left[\operatorname{Pd}\left(h^{\prime} k^{\prime} l^{\prime}\right) \| \mathrm{r}-\mathrm{TiO}_{2}\left(u^{\prime} v^{\prime} w^{\prime}\right)\right]\right\}
\end{aligned}
$$

and consequently

$$
\begin{aligned}
\sigma\left[\operatorname{Pd}(h k l) \| \mathrm{r}-\mathrm{TiO}_{2}(u v w)\right]= & \frac{E_{h k l}^{\mathrm{Pd}}}{1-\nu^{2}} \cdot\left\{\frac{\Delta d\left[\mathrm{Pd}(h k l) \| \mathrm{r}-\mathrm{TiO}_{2}(u v w)\right] / n}{d_{h k l, T=1023 \mathrm{~K}}^{\mathrm{Pd}}}\right. \\
& \left.+\nu \cdot \frac{\Delta d\left[\mathrm{Pd}\left(h^{\prime} k^{\prime} l^{\prime}\right) \| \mathrm{r}-\mathrm{TiO}_{2}\left(u^{\prime} v^{\prime} w^{\prime}\right)\right] / n^{\prime}}{d_{h^{\prime} k^{\prime} l^{\prime}, T=1023 \mathrm{~K}}^{\mathrm{Pd}}}\right\}
\end{aligned}
$$

Here, $h^{\prime}, k^{\prime}, l^{\prime}, u^{\prime}, v^{\prime}$, and $w^{\prime}$ are the Miller indices of the lattice planes being orthogonal to the planes without the dash on the $\mathrm{r}-\mathrm{TiO}_{2}(110)$ surface. Further, $n$ and $n^{\prime}$ are the numbers of Pd planes for which $\Delta d$ was calculated ${ }^{11}, E_{h k l}^{\mathrm{Pd}}$ is Young's modulus of $\mathrm{Pd}$ in $\langle h k l\rangle$ direction and $\nu$ being Poisson's ratio of $\mathrm{Pd}$. In $\mathrm{Pd}$, Young's modulus and Poisson's ratio are isotropic in directions orthogonal to $<111\rangle$, as derived by Wagner [81, chap. 2.3.3]. Thus the following is valid:

$$
E_{\overline{2} 11}^{\mathrm{Pd}}=E_{0 \overline{1} 1}^{\mathrm{Pd}} \approx 136.1 \mathrm{GPa}
$$

Also, Poisson's ratio of $\mathrm{Pd}$ is equal for all directions orthogonal to $<111>$ and has the value $\nu=0.523[81]$.

This results in the following lattice misfit induced stresses:

$$
\sigma\left[\operatorname{Pd}(\overline{2} 11) \| \mathrm{r}-\mathrm{TiO}_{2}(\overline{1} 10)\right]=2.9087 \mathrm{GPa}
$$

and

$$
\sigma\left[\operatorname{Pd}(0 \overline{1} 1) \| \mathrm{r}-\mathrm{TiO}_{2}(001)\right]=1.4230 \mathrm{GPa}
$$

Consequently, the lattice misfit induced mechanical stress is anisotropic. It is tensile with approximately $1.4 \mathrm{GPa}$ for $\mathrm{Pd}(0 \overline{1} 1) \| \mathrm{r}-\mathrm{TiO}_{2}(001)$ and with approximately $2.9 \mathrm{GPa}$ for $\operatorname{Pd}(0 \overline{1} 1) \| \mathrm{r}-\mathrm{TiO}_{2}(001)$.

Considering the thermal induced stresses $\sigma_{\text {thermal }}$ that are calculated in chap. 2.3.1.1, a total stress state in the $\mathrm{Pd}$ film on $\mathrm{r}-\mathrm{TiO}_{2}$ at $\mathrm{RT}$ can finally be estimated:

$$
\begin{aligned}
\sigma_{\text {total }}\left[\operatorname{Pd}(\overline{2} 11) \| \mathrm{r}-\mathrm{TiO}_{2}(\overline{1} 10)\right] & =\sigma\left[\operatorname{Pd}(\overline{2} 11) \| \mathrm{r}-\mathrm{TiO}_{2}(\overline{1} 10)\right]+\sigma_{\text {thermal }}\left[\operatorname{Pd}(\overline{2} 11) \| \mathrm{r}-\mathrm{TiO}_{2}(\overline{1} 10)\right] \\
& =3.505 \mathrm{GPa}
\end{aligned}
$$

and

$$
\begin{aligned}
\sigma_{\text {total }}\left[\operatorname{Pd}(0 \overline{1} 1) \| \mathrm{r}-\mathrm{TiO}_{2}(001)\right] & =\sigma\left[\operatorname{Pd}(0 \overline{1} 1) \| \mathrm{r}-\mathrm{TiO}_{2}(001)\right]+\sigma_{\text {thermal }}\left[\operatorname{Pd}(0 \overline{1} 1) \| \mathrm{r}-\mathrm{TiO}_{2}(001)\right] \\
& =2.194 \mathrm{GPa}
\end{aligned}
$$

\footnotetext{
$\overline{{ }^{11} \text { Therefore } n \text { and } n^{\prime} \text { equal } 2 \text { for } \operatorname{Pd}(\overline{2} 11)} \| \mathrm{r}-\mathrm{TiO}_{2}(\overline{1} 10)$ and $n$ and $n^{\prime}$ equal 15 for $\mathrm{Pd}(0 \overline{1} 1) \| \mathrm{r}-\mathrm{TiO}_{2}(001)$.
} 
The intrinsic stresses appearing in thin films on substrates can be reduced by the introduction of dislocations and interjectional half planes, respectively, at the interface [84, chap. 8.3]. The thermal induced stresses are purely tensile and can thus increase the stresses originating from the lattice misfit, cf. chap. 2.3.1.1.

The lateral strains accompanied by the lateral stresses also affect the strain in normal direction in thin films. The normal direction is free to move and can therefore be considered as free of stress. But a strain $\epsilon_{\text {normal }}$ appears in the normal direction and depends on the lateral strains. $\epsilon_{\text {normal }}$ can be calculated, as derived, by Sander [85, eq. 2.35], for a cubic (111) oriented films, with:

$$
\epsilon_{\text {normal }}=-\frac{C_{11}+2 C_{12}-2 C_{44}}{C_{11}+2 C_{12}+4 C_{44}}\left(\epsilon_{1}+\epsilon_{2}\right)
$$

with the elements of the elastic stiffness tensor $C_{i j}$ and the films lateral strains $\epsilon_{1}$ and $\epsilon_{2}$. Taking the weighted average of the $C_{i j}$ values from Hsu and Leisure [86] and from Rayne [87] gives $C_{11}+2 C_{12}=5.75 \cdot 10^{11} \mathrm{~Pa}$ and $C_{44}=7.15 \cdot 10^{10} \mathrm{~Pa}$. For $\epsilon_{1}$ and $\epsilon_{2}$ the following is valid:

$$
\begin{aligned}
& \epsilon_{1}=\epsilon\left[\operatorname{Pd}(\overline{2} 11) \| \mathrm{r}-\mathrm{TiO}_{2}(\overline{1} 10)\right]+\epsilon_{\text {thermal }}\left[\operatorname{Pd}(\overline{2} 11) \| \mathrm{r}-\mathrm{TiO}_{2}(\overline{1} 10)\right] \approx 0.017 \\
& \epsilon_{2}=\epsilon\left[\operatorname{Pd}(0 \overline{1} 1) \| \mathrm{r}-\mathrm{TiO}_{2}(001)\right]+\epsilon_{\text {thermal }}\left[\operatorname{Pd}(0 \overline{1} 1) \| \mathrm{r}-\mathrm{TiO}_{2}(001)\right] \approx 0.003
\end{aligned}
$$

It follows for $\epsilon_{\text {normal }}$ at RT:

$$
\epsilon_{\text {normal }} \approx-0.010=-1.0 \%
$$

Thus, by using the lateral strains at RT, an compression of $1.0 \%$ is predicted in normal direction. The reason for this is the strong lateral expansion in the $\operatorname{Pd}(\overline{2} 11) \| \mathrm{r}-\mathrm{TiO}_{2}(\overline{1} 10)$ direction.

$\epsilon_{\text {normal }}$ can be measured for thin films using x-ray diffraction (chap. 3.5.1) and it was applied on the prepared Pd films (chap. 4.1.2.1).

In summary, $\mathrm{Pd}$ grows with a (111) orientation on $\mathrm{r}-\mathrm{TiO}_{2}(110)$ surfaces. Considering the known orientation relationship of $\mathrm{Pd}$ on the $\mathrm{r}-\mathrm{TiO}_{2}(110)$ surface, the lattice misfit in the lateral direction of the $\mathrm{Pd} / \mathrm{r}-\mathrm{TiO}_{2}$ interface can be calculated. By assuming $\mathrm{r}$ $\mathrm{TiO}_{2}$ as rigid, a corresponding total lateral stress $\sigma_{\text {total }}$ is calculated. The total stress is anisotropic as the $\mathrm{r}-\mathrm{TiO}_{2}(110)$ surface. $\sigma_{\text {total }}$ is tensile with approximately $3.5 \mathrm{GPa}$ in the $<\overline{2} 11>$ direction and with $2.2 \mathrm{GPa}$ in $<0 \overline{1} 1>$ direction. Considering the high tensile stress in both direction it seems reasonable to assume that dislocations are introduced for thick Pd films in these direction to reduce the total stress state.

\subsubsection{Thermally induced mechanical stress}

In this section the thermal induced stress and strains are estimated that occur by assuming a cooling from $1023 \mathrm{~K}$ to $293 \mathrm{~K}$. 
The thermal expansion equation for $\mathrm{Pd}$ is given in the $T$ range of $293 \mathrm{~K}$ to $1828 \mathrm{~K}$ by Arblaster [72] as follows:

$$
\begin{aligned}
\frac{\delta a}{a_{293 \mathrm{~K}}}(T)= & -3.67831 \cdot 10^{-3}+1.10122 \cdot 10^{-5} \cdot T+2.69121 \cdot 10^{-9} \cdot T^{2} \\
& -2.25680 \cdot 10^{-13} \cdot T^{3}+\frac{6.58134 \cdot 10^{-2}}{T}
\end{aligned}
$$

The temperature dependent lattice constant of $\mathrm{Pd} a(T)$ can be calculated by:

$$
a(T)=\left(1+\frac{\delta a}{a_{293 \mathrm{~K}}}(T)\right) \cdot a_{293 \mathrm{~K}}
$$

In the case of $\mathrm{r}-\mathrm{TiO}_{2}$, the lattice expansion is anisotropic since the lattice is. The temperature dependent lattice parameters $a(T)$ and $c(T)$ are given in $\AA$ from Hummer et al. [83] for a temperature range of approximately $300 \mathrm{~K}$ to $575 \mathrm{~K}$ by:

$$
\begin{aligned}
& a(T)=-6.63642 \cdot 10^{-11} \cdot T^{3}+1.00501 \cdot 10^{-7} \cdot T^{2}-1.00993 \cdot 10^{-5} \cdot T+4.58634 \\
& c(T)=-4.11550 \cdot 10^{-11} \cdot T^{3}+6.40594 \cdot 10^{-8} \cdot T^{2}+4.67561 \cdot 10^{-7} \cdot T+2.95181
\end{aligned}
$$

\begin{tabular}{|c|c|c|c|}
\hline & $293 \mathrm{~K}$ & $1023 \mathrm{~K}$ & $\Delta d$ \\
\hline$d d_{\overline{2} 11}^{\mathrm{Pd}} / \AA$ & 1.5882 & 1.6044 & 0.0162 \\
\hline$d_{\overline{1} 10}^{\mathrm{r}-\mathrm{TiO}_{2}} / \AA$ & 3.2459 & 3.2599 & 0.0140 \\
\hline$d_{0 \overline{1} 11}^{\mathrm{Pd}} / \AA$ & 2.7508 & 2.7789 & 0.0281 \\
\hline$d_{001}^{\mathrm{r}-\mathrm{TiO}}{ }_{2} / \AA$ & 2.9564 & 2.9753 & 0.0189 \\
\hline
\end{tabular}

From equations 2.9, 2.10 and 2.11 the lattice plane distances for the orientation relationship can be calculated at $1023 \mathrm{~K}$ and at $293 \mathrm{~K}$, as summarized in tab. 2.4. As

Table 2.4.: $T$ dependent lattice plane distances in $\mathrm{Pd}$ and $\mathrm{r}-\mathrm{TiO}_{2}$. The specific planes are chosen from the orientation relationship of $\mathrm{Pd}$ on $\mathrm{r}-\mathrm{TiO}_{2}$, cf. chap. 2.3.1. The difference in the lattice plane distance $\Delta d$ between $293 \mathrm{~K}$ and $1023 \mathrm{~K}$ is shown.

can be seen from the difference $\Delta d$ values in tab. 2.4, the Pd lattice likes to relax more than the $\mathrm{r}-\mathrm{TiO}_{2}$ lattice. The $\mathrm{r}-\mathrm{TiO}_{2}$ substrate can be assumed to be rigid and thus the Pd lattice cannot totally relax. A thermal induced net strain $\epsilon_{\text {thermal }}$ remains in $\mathrm{Pd}$ and can be calculated via:

$$
\epsilon_{\text {thermal }}\left[\operatorname{Pd}(h k l) \| \mathrm{r}-\mathrm{TiO}_{2}(u v w)\right]=\frac{\Delta d_{h k l}^{\mathrm{Pd}}-\Delta d_{u v w}^{\mathrm{r}-\mathrm{TiO}_{2}}}{d_{h k l, T=293 \mathrm{~K}}^{\mathrm{Pd}}}
$$


This yields

$$
\epsilon_{\text {thermal }}\left[\operatorname{Pd}(\overline{2} 11) \| \mathrm{r}-\mathrm{TiO}_{2}(\overline{1} 10)\right]=0.0014=0.14 \%
$$

and

$$
\epsilon_{\text {thermal }}\left[\operatorname{Pd}(0 \overline{1} 1) \| \mathrm{r}-\mathrm{TiO}_{2}(001)\right]=0.0034=0.34 \%
$$

The thermal induced mechanical stress can be derived from these strains by [81]

$$
\begin{array}{r}
\sigma_{\text {thermal }}\left[\operatorname{Pd}(\overline{2} 11) \| \mathrm{r}-\mathrm{TiO}_{2}(\overline{1} 10)\right]=\frac{E_{211}^{\mathrm{Pd}}}{1-\nu^{2}} \cdot\left\{\epsilon_{\text {thermal }}\left[\operatorname{Pd}(\overline{2} 11) \| \mathrm{r}-\mathrm{TiO}_{2}(\overline{1} 10)\right]\right. \\
\left.+\nu \cdot \epsilon_{\text {thermal }}\left[\operatorname{Pd}(0 \overline{1} 1) \| \mathrm{r}-\mathrm{TiO}_{2}(001)\right]\right\}
\end{array}
$$

and

$$
\begin{aligned}
& \sigma_{\text {thermal }}\left[\operatorname{Pd}(0 \overline{1} 1) \| \mathrm{r}-\mathrm{TiO}_{2}(001)\right]=\frac{E_{0 \overline{1} 1}^{\mathrm{Pd}}}{1-\nu^{2}} \cdot\left\{\nu \cdot \epsilon_{\text {thermal }}\left[\operatorname{Pd}(\overline{2} 11) \| \mathrm{r}-\mathrm{TiO}_{2}(\overline{1} 10)\right]\right. \\
& \left.+\epsilon_{\text {thermal }}\left[\operatorname{Pd}(0 \overline{1} 1) \| \mathrm{r}-\mathrm{TiO}_{2}(001)\right]\right\}
\end{aligned}
$$

with $\nu=0.523$ [81] being Poisson's ratio of $\mathrm{Pd}$ orthogonal to $<111>$.

Thus, the thermal induced mechanical stresses induced by cooling the $\mathrm{Pd} / \mathrm{r}-\mathrm{TiO}_{2}$ system from $1023 \mathrm{~K}$ to $293 \mathrm{~K}$ are

$$
\sigma_{\text {thermal }}\left[\operatorname{Pd}(\overline{2} 11) \| \mathrm{r}-\mathrm{TiO}_{2}(\overline{1} 10)\right]=596.3 \mathrm{MPa}
$$

and

$$
\sigma_{\text {thermal }}\left[\operatorname{Pd}(0 \overline{1} 1) \| \mathrm{r}-\mathrm{TiO}_{2}(001)\right]=771.2 \mathrm{MPa}
$$

As it can be seen from this results, $\sigma_{\text {thermal }}$ is tensile in nature and is anisotropic (in lateral direction of the $\mathrm{Pd}$ film).

In summary, a tensile thermal induced mechanical stress $\sigma_{\text {thermal }}$ occurs in the $\mathrm{Pd}$ film on $\mathrm{r}-\mathrm{TiO}_{2}$ when cooling from $1023 \mathrm{~K}$ to $293 \mathrm{~K}$. The stress is anisotropic due to the anisotropic thermal expansion and contraction of the $\mathrm{r}-\mathrm{TiO}_{2}$ lattice. Tensile stresses of up to approximately $800 \mathrm{MPa}$ are possible.

\subsubsection{Electrical properties of metal/semiconductor contacts (MSC)}

This section briefly discusses the electronic properties of a metal/semiconductor contact (MSC). The concept is then applied qualitatively to the $\mathrm{Pd} / \mathrm{r}-\mathrm{TiO}_{2}$ system. A comprehensive introduction to MSC is given by Mönch [88]. A recent review on MSCs was given by Tung [89]. As mentioned before, $\mathrm{r}-\mathrm{TiO}_{2}$ is expected to be in a $n$-type condition state, so the focus in the following is on metal/n-type semiconductor interfaces. 
A metal and a semiconductor can have different electron chemical potentials $\mu_{e}$. Connecting both of them in a metal/semiconductor contact yields to an electron exchange at the interface. A space charge and depletion layer builds up in the semiconductor. This leads to a new equilibrium state that typically includes band bending at the semiconductor site. This electronic state of a metal $/ n$-type semiconductor contact is sketched in fig. 2.15a.

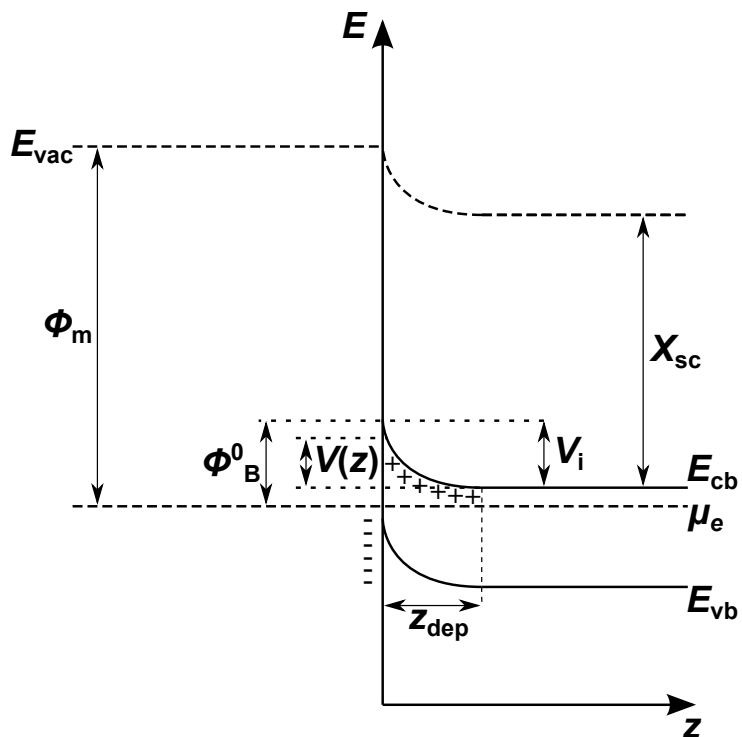

(a) Sketch of the band alignment at a metal $/ n$ type semiconductor contact (MSC). The metal on the left is connected to the semiconductor on the right. The contact of both sides results in an electron exchange from the semiconductor to the metal. Hence, a depletion layer forms which is accompanied by a upwards band bending. The symbols refer to the imaginary vacuum energy $E_{\mathrm{vac}}$, the electron chemical potential $\mu_{e}$, the energy of the conduction band minimum $E_{\mathrm{cb}}$ and valence band maximum $E_{\mathrm{vb}}$, respectively, the work function of the metal $\Phi_{\mathrm{m}}$, the electron affinity of the semiconductor $X_{\mathrm{sc}}$, the Schottky barrier height $\Phi_{\mathrm{B}}^{0}$, the interface band bending $V_{\mathrm{i}}$, the band bending at the distance $z$ to the interface and the extension of the depletion layer $z_{\mathrm{dep}}$.

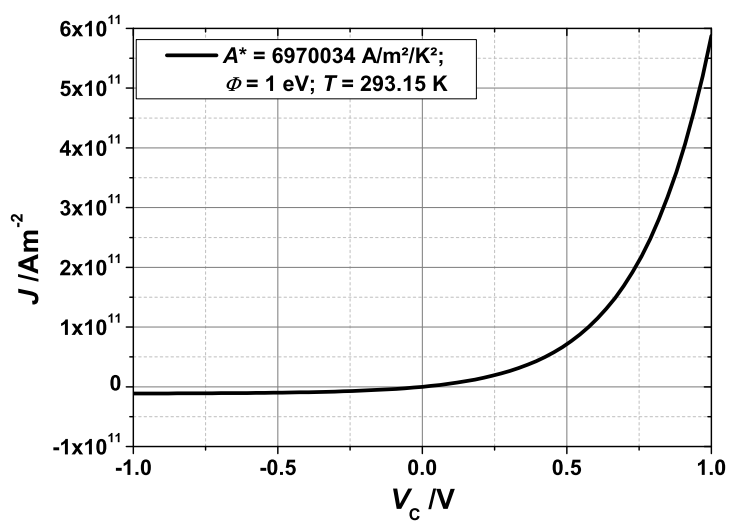

(b) Typical current-voltage $(I-V)$ characteristic of an MSC. $V_{\mathrm{C}}$ gives the voltage that drops over the contact and $J$ is the to $I$ related current density. The characteristic reveals a diode like behavior with a forward current direction for positive voltage and with a reverse direction for negative voltage.

\section{Figure 2.15.}

The Schottky-Mott rule is based on an electrostatics description of the MSC [89] and can be used to estimate the Schottky barrier height that is established at the interface, cf. fig. 2.15a:

$$
\Phi_{\mathrm{B}}^{0}=\Phi_{\mathrm{m}}-X_{\mathrm{sc}}
$$


Here, $\Phi_{\mathrm{m}}$ is the work function of the metal and $X_{\mathrm{sc}}$ is the electron affinity of the semiconductor.

For the $\mathrm{Pd} / \mathrm{r}-\mathrm{TiO}_{2}$ system, the work function of $\mathrm{Pd} \Phi_{\mathrm{Pd}}$ is $4.99 \mathrm{eV}$ [74] and the electron affinity of $\mathrm{r}-\mathrm{TiO}_{2} X_{\mathrm{r}-\mathrm{TiO}_{2}}$ is $4.2 \mathrm{eV}$ for the (110) surface [29]. Thus $\Phi_{\mathrm{Pd}}>$ $X_{\mathrm{r}-\mathrm{TiO}_{2}}$ and it follows according to eq. 2.12:

$$
\Phi_{\mathrm{B}}^{0}=\Phi_{\mathrm{Pd}}-X_{\mathrm{r}-\mathrm{TiO}_{2}}=790 \mathrm{meV}
$$

Thus a situation as depicted in fig. 2.15a can be expected for the $\mathrm{Pd} / \mathrm{r}-\mathrm{TiO}_{2}$ system.

Experimental barrier heights for the $\mathrm{Pd} / \mathrm{r}-\mathrm{TiO}_{2}$ system were extracted with $I$ $V$ measurements. Yamamoto et al. [10] found a Schottky barrier height for $\mathrm{Pd} / \mathrm{r}$ $\mathrm{TiO}_{2}(001)$ of $(670 \pm 50) \mathrm{meV}$.

Applying a voltage to a MSC and thus to the band picture sketched in fig. 2.15a results in a typical current-voltage $(I-V)$ characteristic which is depicted in fig. 2.15b. For positive voltages the electrons flow from the semiconductor to the metal side. The increasing (external) voltage decreases the Schottky barrier height and increases the current flows. This direction is called the forward current. For negative voltages, the electrons flow from the metal to the semiconductor side. The decreasing (external) voltage increases the Schottky barrier height allowing less electrons to pass over the barrier. This direction is called the reverse direction. The qualitative shape of the $I-V$ characteristic is valid for all MSCs. It is important to note that the $I-V$ characteristic can change in a hydrogen environment, as discussed in chap. 2.3.2.1. The $I-V$ and $J-V$ characteristic, respectively, is typically written by a thermionic characteristic $[88$, eq. 3.29]:

$$
J_{\text {te }}=A_{\mathrm{R}}^{*} T^{2} \exp \left(-\frac{\Phi_{\mathrm{B}}^{0}}{k_{\mathrm{B}} T}\right) \exp \left(\frac{e_{0} V_{\mathrm{C}}}{k_{\mathrm{B}} T}\right) \cdot\left[1-\exp \left(-\frac{e_{0} V_{\mathrm{C}}}{k_{\mathrm{B}} T}\right)\right]
$$

With $A_{\mathrm{R}}^{*}$ being the effective Richardson constant and $J_{\mathrm{te}}$ being the thermionic current density. This characteristic is presented in fig. 2.15b.

An ohmic (back) contact to $\mathrm{r}-\mathrm{TiO}_{2}$ was established with different materials in the past. For example, Cheste et al. used gallium [56], Yamamoto et al. [10] used indium, Hope and Bard [90] and Kobayashi et al. [11] used indium-gallium, and Cerchez et al. [12] used titanium.

\subsubsection{Influence of hydrogen on metal $/ \mathrm{r}-\mathrm{TiO}_{2}$ contacts}

The interaction of hydrogen with MSCs is of interest in the fields of gas sensing and microelectronics. In both applications, a hydrogen interaction alters the properties of the corresponding device. While this is wanted for gas sensing, this is not wanted in microelectronics. Thus a lot of studies are available on MSCs with respect to those fields.

The interaction of $\mathrm{Pd} / \mathrm{r}-\mathrm{TiO}_{2}$ with hydrogen and its influence on the $I-V$ characteristic was studied in the field of gas sensing $[10,11]$. 
Yamamoto et al. [10] studied the $\mathrm{Pd} / \mathrm{r}-\mathrm{TiO}_{2}(001)$ system with measurements of the $I$ - $V$ characteristic. They prepared $\mathrm{r}-\mathrm{TiO}_{2}$ crystals by annealing at $773 \mathrm{~K}$ for $1 \mathrm{~h}$ in vacuum $(\sim 0.05 \mathrm{~Pa})$. This suggests their $\mathrm{r}-\mathrm{TiO}_{2}$ were in the reduced regime, with $\mathrm{V}_{\mathrm{O}}$ being the dominant defect, cf. chap. 2.1.3. They measured the system in air and subsequently added more and more hydrogen to the air. The current flowing at a particular voltage increased with increasing amounts of hydrogen in air up to a point where the typical MSC $I-V$ characteristic changed to a nearly ohmic behavior. Their measurements are shown in fig. 2.16.

Figure 2.16.: $I-V$ characteristic of a $\mathrm{Pd} / \mathrm{r}-\mathrm{TiO}_{2}(001)$ contact in (a) air and in (b-g) hydrogen-containing air at $298 \mathrm{~K}$. The partial pressure of hydrogen increases from (b) $14 \mathrm{ppm}$ to (g) $1.5 \%$ (red arrow). The presence of hydrogen significantly increases the flowing current. Adapted with permission from Yamamoto et al. [10]. Copyright 1980 Elsevier.

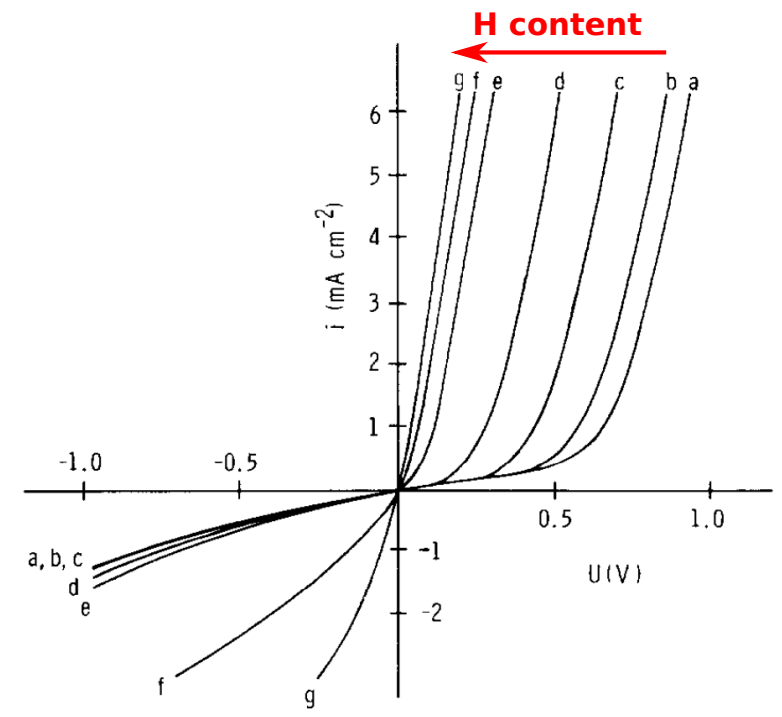

The flowing current $I$ is plotted against the applied voltage $V$. In air (a) a, more or less, typical $I-V$ characteristic are observed. Increasing the hydrogen concentrations yields higher flow currents at the same voltage. This continues up to a point at which the $I-V$ characteristic becomes nearly ohmic (g). This measurement showed the $\mathrm{Pd} / \mathrm{r}-$ $\mathrm{TiO}_{2}$ system to be suitable for gas sensor applications. Yamamoto et al. suggested that the barrier height is reduced in the presence of $\mathrm{H}$, due to a hydrogen induced change of the $\mathrm{Pd}$ work function $\Phi_{\mathrm{Pd}}$. Yamamoto et al. observed that the $\mathrm{Pd} / \mathrm{r}-\mathrm{TiO}_{2}$ systems needs approximately $5 \mathrm{~min}$ at $298 \mathrm{~K}$ to show the new $I-V$ characteristic after the hydrogen amount was increased. Yamamoto et al. also prepared MSCs with other metals in combination with $\mathrm{r}-\mathrm{TiO}_{2}$. For example, they used platinum $(\mathrm{Pt})$, gold $(\mathrm{Au})$ and nickel $(\mathrm{Ni})$. The sensitivity to hydrogen for Pd was found to be the highest [10]. Consequently, $\mathrm{Pd}$ is a suitable material for this work.

Kobayashi et al. [11] studied both the $\mathrm{Pd} / \mathrm{r}-\mathrm{TiO}_{2}(001)$ and the $\mathrm{Pd} / \mathrm{r}-\mathrm{TiO}_{2}(100)$ systems ${ }^{12}$. They measured $I-V$ and capacitance-voltage $(C-V)$ characteristics on these contacts and the work function of $\mathrm{Pd}$ with a Kelvin probe at RT in various amounts of hydrogen in air. In accordance with the Yamamoto et al. [10] results, Kobayashi et al. found a lowering of the interface barrier in a hydrogen atmosphere. Kobayashi et al.

\footnotetext{
${ }^{12}$ By electron beam evaporation $20 \mathrm{~nm}$ of $\mathrm{Pd}$ were deposited in a vacuum on the $\mathrm{r}-\mathrm{TiO}_{2}$ single crystals.
} 
build up a chemical reaction model and evaluated their measurements based on this model. They developed the following qualitative picture of the hydrogen interaction with their $\mathrm{Pd} / \mathrm{r}-\mathrm{TiO}_{2}$ contacts, which is shown in fig. 2.17. In the initial state in

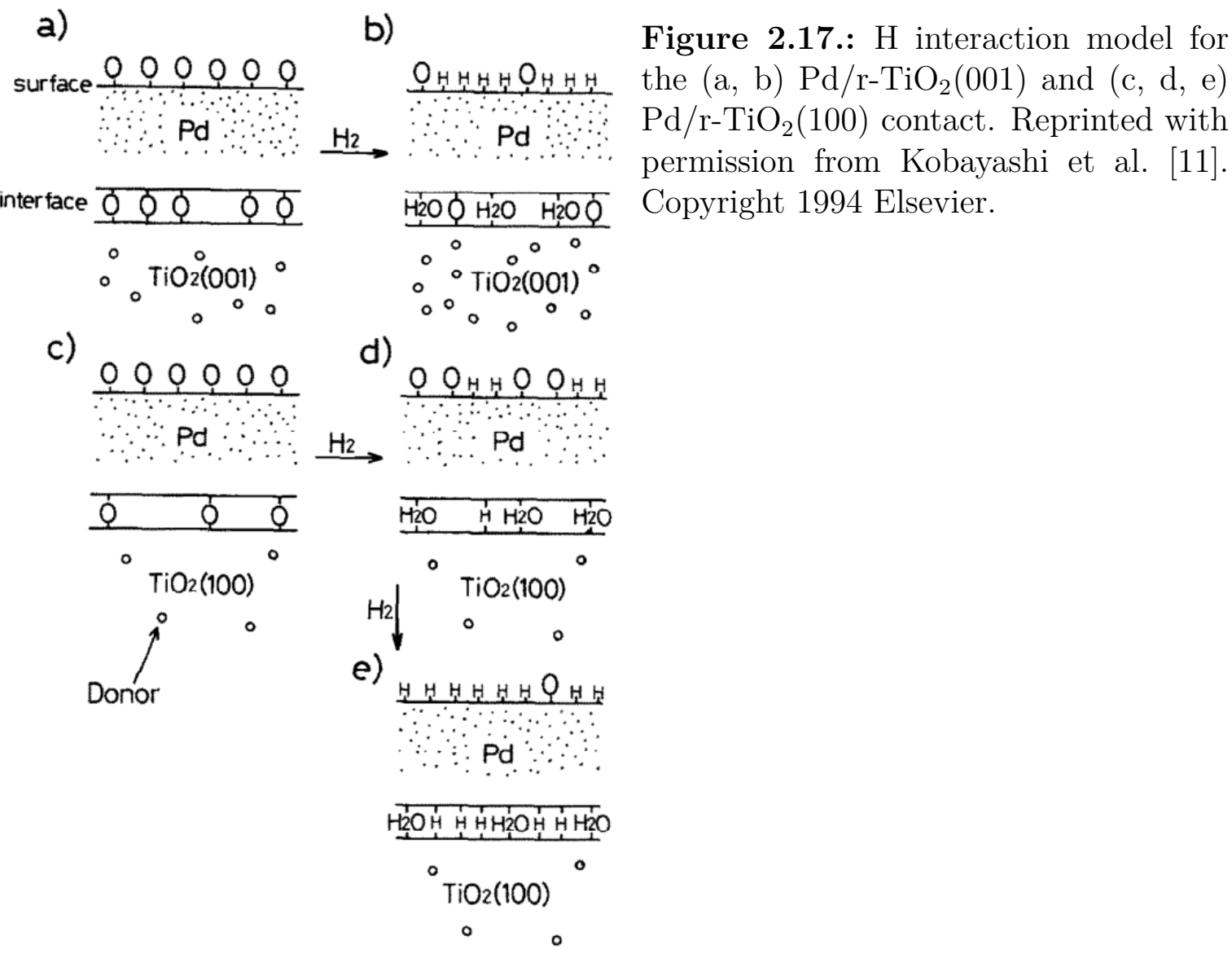

air (cf. fig. 2.17 a and c) the $\mathrm{Pd} / \mathrm{r}-\mathrm{TiO}_{2}(001)$ and the $\mathrm{Pd} / \mathrm{r}-\mathrm{TiO}_{2}(100)$ contacts are similar. The $\mathrm{Pd}$ surface is covered with dissociated oxygen. At the $\mathrm{Pd} / \mathrm{r}-\mathrm{TiO}_{2}$ interface some $\mathrm{Pd}$ is connected to $\mathrm{O}$, some is free of it. This situation changes in hydrogen environment. On the $\mathrm{Pd}$ surface, some of the $\mathrm{O}$ reacts to from $\mathrm{H}_{2} \mathrm{O}$ and leaves the surface. The free sites are than occupied by dissociatively adsorbed hydrogen [11] (cf. fig. $2.17 \mathrm{~b}$ and d). At the interface, two reaction channels are expected: the first being the adsorption of hydrogen on free $\mathrm{Pd}$ sites and the second being interfacial $\mathrm{H}_{2} \mathrm{O}$ formation on $\mathrm{Pd}-\mathrm{O}$ sites. Kobayashi et al. [11] suggest that $\mathrm{H}_{2} \mathrm{O}$ formation is dominant for the $\mathrm{Pd} / \mathrm{r}-\mathrm{TiO}_{2}(001)$ contact in the whole hydrogen regime while for the $\mathrm{Pd} / \mathrm{r}-\mathrm{TiO}_{2}(100)$ contact this is true up to approximately $100 \mathrm{ppm}$ of $\mathrm{H}$ (cf. fig. $2.17 \mathrm{~b}$ and d). Above $100 \mathrm{ppm}$, a preferred adsorption of hydrogen at the interface was found for $\mathrm{Pd} / \mathrm{r}-\mathrm{TiO}_{2}(100)$ (cf. fig. $2.17 \mathrm{e}$ ). Kobayashi et al. found indications that for the $\mathrm{Pd} / \mathrm{r}-\mathrm{TiO}_{2}(001)$ contact hydrogen diffuses in the depletion layer of $\mathrm{r}-\mathrm{TiO}_{2}$ and there hydrogen increases the doping density (cf. fig. $2.17 \mathrm{~b}$ ). This behavior was not found 
for the $\mathrm{Pd} / \mathrm{r}-\mathrm{TiO}_{2}(100)$ contact.

Kobayashi et al. [11] additionally observed that the $\mathrm{Pd} / \mathrm{r}-\mathrm{TiO}_{2}(100)$ contact was more sensitive to $\mathrm{H}$ : the $I-V$ characteristic already revealed changes at $10 \mathrm{ppm}$ hydrogen while for the $\mathrm{Pd} / \mathrm{r}-\mathrm{TiO}_{2}(001)$, this was not the case until $300 \mathrm{ppm}$ hydrogen. At $1 \%$ hydrogen, both contact types became ohmic. Thus the Schottky barrier is significantly lowered in this condition. Kobayashi et al. state, in accordance with Yamamoto et al. [10], that after a few minutes the new $I-V$ characteristic was established. Exchanging hydrogen with $101,325 \mathrm{~Pa}$ of $\mathrm{O}$ reversed the changes, decreasing the conductivity, but the kinetics differed, as the new equilibrium was reached after $50 \mathrm{~h}$. Kobayashi et al. suggest the slower kinetics originate from a low rate of $\mathrm{O}$ reaction at the $\mathrm{Pd} / \mathrm{r}-\mathrm{TiO}_{2}$ interface.

It is important to note that Kobayashi et al. [11] prepared their samples by reducing the $\mathrm{r}-\mathrm{TiO}_{2}$ crystals in a $\mathrm{H}_{2}$ atmosphere at approximately $953 \mathrm{~K}$. Based on the discussions in chap. 2.1.4 and 4.4 this can yield in $\mathrm{H}_{\mathrm{O}}$ being the dominant defect species rather than $\mathrm{V}_{\mathrm{O}}$.

Cerchez et al. [12] and Strungaru et al. [13] studied MSCs of Pt and nanoporous (np) $\mathrm{TiO}_{2}$ in hydrogen environment. Cerchez et al. summarized the recent picture about the hydrogen interaction with the $\mathrm{Pt} / \mathrm{np}-\mathrm{TiO}_{2}$ system [12]: (As $\mathrm{Pd}$ ) the $\mathrm{Pt}$ dissociates the $\mathrm{H}_{2}$ molecule to atomic hydrogen and transports it to the interface. There, the hydrogen spills over into the $\mathrm{np}-\mathrm{TiO}_{2}$ and acts as a donor predominantly at pore surfaces. Hydrogen is expected to enrich, especially at the $\mathrm{Pt} / \mathrm{np}-\mathrm{TiO}_{2}$ interface, where it is assumed to reduce the Schottky barrier height. This is in qualitative agreement with the observations of Yamamoto et al. [10] and Kobayashi et al. [11] for the $\mathrm{Pd} /$ single crystal $\mathrm{r}-\mathrm{TiO}_{2}$ system in $\mathrm{H}$, where they found a predominant influence of the interface and interface near region. It is stated by Cerchez et al. [12] that the underlying mechanisms are unknown and that no picture of the microscopic processes has been established.

Cerchez et al. [12] measured time dependent $I-V$ and $C-V$ characteristics of the $\mathrm{Pt} / \mathrm{np}-\mathrm{TiO}_{2}$ contacts in gas flow of $\mathrm{H}_{2}$ and $\mathrm{N}_{2} . \mathrm{H}_{2}$ concentrations from $0 \mathrm{ppm}$ to $200 \mathrm{ppm}$ were used in their experiments. Based on the given parameters for the sample preparation, it is not possible to judge which oxygen activity regime, cf. chap. 2.1.3, is appropriate to describe the np- $\mathrm{TiO}_{2}$ conditions. The contacts revealed a fast response for the high hydrogen concentration regime and a slow response for the low hydrogen concentration regime. Therefore, Cerchez et al. [12] studied Pt/np- $\mathrm{TiO}_{2}$ contacts at $30 \mathrm{ppm}$ as a function of time (up to $1200 \mathrm{~min}$ ) to observe possible mechanisms that result in the finally reduced conductivity of the contacts. Cerchez et al. evaluated their $I$ - $V$ measurements based on an equivalent circuit model that is sketched in fig. 2.18. It is shown that the diode has a parallel resistance $R_{\mathrm{p}}$ which allows a current $I_{\mathrm{p}}$ to circumvent the diode. Applying the model from fig. 2.18 to their time dependent $I-V$ characteristic measurements and by measuring $C-V$ characteristics Cerchez et al. [12] found that $R_{\mathrm{p}}$ decreases with time. Also, the doping density of the MSC increases and the MSC's contact area decreases with time. Based on these results Cerchez et 


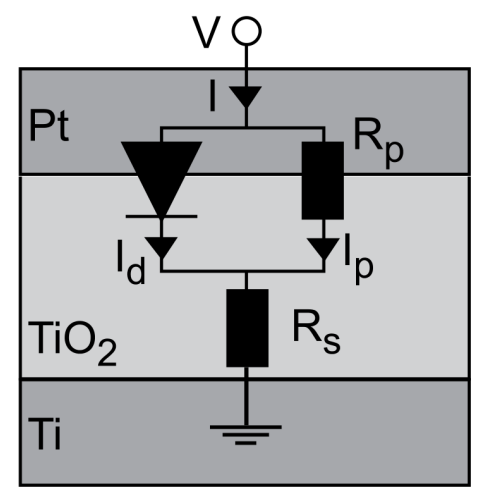

Figure 2.18.: Sketch of a equivalent circuit model to fit $I$ - $V$ characteristics. The parallel resistance $R_{\mathrm{p}}$ allows a current $I_{\mathrm{p}}$ to bypass the MSC and diode, respectively. Reprinted with permission from Cerchez et al. [12]. Copyright 2013 AIP Publishing LLC.

al. [12] concluded that the interface transforms inhomogeneously. Some parts of the contact get resistive under hydrogen exposure (cf. $R_{\mathrm{p}}$ in fig. 2.18). The others (still diode like, cf. fig. 2.18) trap hydrogen at the interface where it is incorporated as a donor-like state [12]. Cerchez et al. [12] see the origin of the change of the diode-like parts as an increase of the charge neutrality level which results in a reduction of the Schottky barrier height and in an increased conductivity of the MSC.

Strungaru et al. [13] studied the process of electroforming, also known as 'memristive behavior', for $\mathrm{Pt} / \mathrm{np}-\mathrm{TiO}_{2}$ contacts in hydrogen environment. Without hydrogen no electroforming was found for electric fields below $4 \cdot 10^{5} \mathrm{~V} / \mathrm{m}$. Above that a clear electroforming behavior was found from Strungaru et al. in measured $I-V$ characteristics. They found that the presence of hydrogen accelerated the observed electroforming process. Strungaru et al. explained the electroforming process by the formation of conductive filaments originating from the $\mathrm{Pt} / \mathrm{np}-\mathrm{TiO}_{2}$ interface. These filaments can break through the Schottky barrier and can be an explanation for the inhomogeneous interface change observed by Cerchez et al. [12]. The filaments were suggested to consist of a Magnéli phase or hydrogen-oxygen vacancy complexes [13]. Strungaru et al. [13] stated that the microscopic origin is still unclear.

In summary, $\mathrm{Pd} / \mathrm{r}-\mathrm{TiO}_{2}$ contacts are a suitable material system to study the hydrogen interaction since they show high hydrogen sensitivity when compared to $\mathrm{Pt} / \mathrm{r}-\mathrm{TiO}_{2}$ or $\mathrm{Au} / \mathrm{r}-\mathrm{TiO}_{2}$ contacts [10]. The $\mathrm{r}-\mathrm{TiO}_{2}(110)$ crystals chosen have an orthogonal alignment to the $c$-axis as did the $\mathrm{Pd} / \mathrm{r}-\mathrm{TiO}_{2}(100)$ system studied by Kobayashi et al. [11] Therefore, a similar behavior is expected and as well as a comparably high hydrogen sensitivity. The hydrogen interaction with $\mathrm{Pd} / \mathrm{r}-\mathrm{TiO}_{2}(100)$ contacts was found to be dominated by interface effects such as $\mathrm{H}_{2} \mathrm{O}$ formation and hydrogen adsorption. The kinetics of the hydrogen induced changes of $\mathrm{Pd} / \mathrm{r}-\mathrm{TiO}_{2}$ contacts are expected to be fast, and were observed to occur within five minutes [10,11]. Thus an equilibration time of $1 \mathrm{~h}$ in a hydrogen environment seems reasonable, cf. chap. 3.2.2. Experiments performed by Cerchez et al. [12] and Strungaru et al. [13] on Pt/nanoporous- $\mathrm{TiO}_{2}$ in hydrogen environment suggest two processes taking place at the interface: a homogeneous change that increases the general conductivity, probably due to a lowering 
of the Schottky barrier height, and an inhomogeneous change that locally results in resistive paths through the Schottky barrier. No underlying microstructural picture was established $[12,13]$ for these findings. 



\section{Experimental methods and advanced EELS methodology}

To get new insights into the hydrogen interaction with the $\mathrm{Pd} / \mathrm{r}-\mathrm{TiO}_{2}$ system and its interface vicinity region in the $\mathrm{r}-\mathrm{TiO}_{2}$, a new in situ electron energy loss spectroscopy (EELS) methodology in combination with local scanning transmission electron microscopy (STEM) was developed and applied on the material system. A precise experimental set-up within the environmental TEM (ETEM) and the fabrication of high quality TEM lamella were necessary requirements for the EELS measurements and are shown in chap. 3.2 .

A special focus was laid on reliable EELS experiments at the $\mathrm{Pd} / \mathrm{r}-\mathrm{TiO}_{2}$ interface. A very important element of the performed EELS measurements was the high energy stability, and accompanying high energy resolution. A methodology was developed to evaluate the considerable number $(\sim 10.000)$ of EELS spectra in a reliable and reproducible way. Details of why and how EELS acquisition parameters were chosen will be presented in chap. 3.3. In thin chapter the developed EELS methodology is presented as well. In addition to the EELS experiments, positron annihilation spectroscopy (PAS) was performed (see chap. 3.4) to experimentally assess the influence of Ti related defects in this material system. To understand hydrogen related changes in the DoS of $\mathrm{r}-\mathrm{TiO}_{2}$, density functional theory (DFT) calculations were carried out as described in chap. 3.8.

The produced $\mathrm{Pd} / \mathrm{r}-\mathrm{TiO}_{2}$ samples were characterized with X-ray investigations, $\mathrm{x}-$ ray photoelectron spectroscopy and atomic force microscopy which are described in chap. 3.5, 3.6 and 3.7, respectively.

The focus were Pd films with thicknesses of $\sim 100 \mathrm{~nm}$. To successfully perform suitable EELS experiments on these samples, a high crystallinity, a low electronic, and low microstructural contributions were required to create a sharp interface of the $\mathrm{Pd}$ film to the $\mathrm{r}-\mathrm{TiO}_{2}$ crystal. The $\sim 100 \mathrm{~nm} \mathrm{Pd}$ films were investigated with electron energy loss spectroscopy and positron annihilation spectroscopy to get insights in the underlying mechanisms of the hydrogen interaction with the $\mathrm{Pd} / \mathrm{r}-\mathrm{TiO}_{2}$ system.

During this work, Pd films with thicknesses of $\sim 10 \mathrm{~nm}$ were also produced. These Pd films allowed for high lateral stress states, since stress reduction by the incorporation of dislocations at the interface can be prohibited in sufficiently thin films [91]. Though the $\sim 10 \mathrm{~nm}$ Pd films were not the focus of this thesis, their deposition parameters and characterization are included for the sake of completeness, see appendix A.2. 


\subsection{Magnetron sputtering}

In ion beam sputtering, high voltage is used to spark an ion plasma of a working gas. For the sputtering process, the working gas is accelerated towards a target material where atoms are knocked out due to the inelastic collision between the working gas ions and the target atoms. The target material is then deposited on a substrate for film growth. Argon (Ar) is a commonly used working gas and was used in this study. As distinct from a Kaufmann source, a magnetron sputter system adds a magnetic field that increases the plasma density due to a circular flight path of the working gas ions. In addition to the working gas, an additional reactive gas, such as oxygen, can be added. This gas can react with the target material within the target or on the substrate. This technique is called reactive sputtering, and it allows oxides to be deposited from pure metal targets. Reactive conditions can also be used to prevent or reduce oxygen loss from the substrate material.

In the magnetron sputter system used, the magnetic poles have different strengths. The corresponding magnetic field lines do not close within the target surface. A larger volume in which the plasma density is enhanced results due to the presence of this magnetic field. This type is called unbalanced magnetron sputtering [92].

\subsubsection{Pretreatment of the $\mathrm{r}-\mathrm{TiO}_{2}$ crystals}

The quality of the $\mathrm{Pd} / \mathrm{r}-\mathrm{TiO}_{2}$ interface was of significant importance for the EELS measurements. Therefore a pretreatment for the $\mathrm{r}-\mathrm{TiO}_{2}$ crystals was developed in collaboration with Niklas Herwig [93] to improve the interface sharpness. The pretreatment procedure is based on the work of Shimizu et al. [94] and Nakamura et al. [95], but was further refined. The procedure is:

- The initial $\mathrm{r}-\mathrm{TiO}_{2}$ substrates were etched for $10 \mathrm{~min}$ in a hydrofluoric acid (HF) solution of $20 \%$.

- The etched substrates were cleaned by distilled water and softly dried with a laboratory wipe (Kimtech Science*).

- Subsequently, the etched substrates were annealed at $1173 \mathrm{~K}$ for $1 \mathrm{~h}$ in a furnace with oxygen gas flow of more than $100 \mathrm{kPa}^{1}$. The samples were cooled in the oxygen flow until their temperature was below $573 \mathrm{~K}$ (at least $6 \mathrm{~h}$ ).

The $\mathrm{r}-\mathrm{TiO}_{2}(110)$ single crystals (CrysTec GmbH, Berlin, Germany) were single-sided epi-polished, with dimensions of $10 \times 10 \times 0.5 \mathrm{~mm}^{3}$ and with the one edge parallel to the (001) crystallographic plane to a tolerance (miscut) below $0.1^{\circ}$.

\footnotetext{
${ }^{1}$ Due to the construction of the furnace, the oxygen pressure had to be a bit higher than the atmospheric pressure to ensure high oxygen concentration in the flow furnace. This was especially important at temperatures above $1173 \mathrm{~K}$ to prevent the $\mathrm{r}-\mathrm{TiO}_{2}$ from nitrating.
} 


\subsubsection{Oxygen treatment and Palladium film deposition}

The $\mathrm{Pd}$ films were produced on the pretreated $\mathrm{r}-\mathrm{TiO}_{2}(110)$ crystals in two steps. First the substrates were cleaned (again) within the sputtering system via thermal annealing, and, subsequently, the Pd films were deposited.

In the first step, the substrates were heated to the target temperature at a rate not higher than $1 \mathrm{~K} / \mathrm{s}$. Typically the substrates were kept for $20 \mathrm{~min}$ at $1023 \mathrm{~K}$ (pyrometer) in oxygen to clean the $\mathrm{r}-\mathrm{TiO}_{2}$ surface of gas residues [94] and to establish the defect equilibrium for the desired reduced regime, cf. chap. 2.1.3 and especially the red indicated line in fig. 2.10a: For that, an oxygen partial pressure of $7 \cdot 10^{-3} \mathrm{~Pa}$ was chosen. The $\mathrm{r}-\mathrm{TiO}_{2}$ crystal can be assumed to be in a $n$-type conducting state.

In the second step, the deposition temperature was set and the Pd sputtering process started. A pre-sputtering step was performed for 5 min to clean the Pd target. The shutter in front of the source was closed during pre-sputtering, resulting in no $\mathrm{Pd}$ deposition. Subsequently, the $\mathrm{Pd}$ film was deposited on the reduced $\mathrm{r}-\mathrm{TiO}_{2}$ crystal. After sputtering, the samples were cooled in $50 \mathrm{~K}$ steps to room temperature (RT). After each temperature step, the temperature was held for $1 \mathrm{~min}$. This was done down to $573 \mathrm{~K}$; below that, the sample cooling rate was low.

For the Pd film deposition, the background pressure was typically on the order of $10^{-5} \mathrm{~Pa}$. The Ar flux was set to $49.8 \mathrm{sccm}$ which corresponds to a (partial) pressure of $2.4 \cdot 10^{-1} \mathrm{~Pa}$. For the thick Pd films, an oxygen flux of $1.81 \mathrm{sccm}$ was chosen, resulting in a partial pressure of $7 \cdot 10^{-3} \mathrm{~Pa}$. The thick $\mathrm{Pd}$ films were deposited with a sputter power of $20 \mathrm{~W}$; the thin $\mathrm{Pd}$ films with a power of $10 \mathrm{~W}$. Sputter rates of $11.8 \mathrm{~nm} / \mathrm{min}$ at $20 \mathrm{~W}$ and of $4 \mathrm{~nm} / \mathrm{min}$ at $10 \mathrm{~W}$ resulted. The deposition temperature, $T_{\mathrm{D}}$, was $1023 \mathrm{~K}$ (pyrometer) for the thick Pd films and RT for the thin Pd films. An overview of the prepared $\mathrm{Pd}$ films with the chosen deposition parameters is presented in chap. 4.1.2 and summarized in Tab. 4.1.

The Pd target material used was bought from the Allgemeine Gold- und Silberscheideanstalt AG (Pforzheim, Germany), had a purity of $99.95 \%$ and dimensions of $\varnothing 50.8 \mathrm{~mm} \times 1 \mathrm{~mm}$. The $\mathrm{Pd}$ films were deposited on the pretreated $\mathrm{r}-\mathrm{TiO}_{2}$ crystals.

Photos of the used magnetron sputter system are depicted in fig. 3.1a for the exterior and $3.1 \mathrm{~b}$ for the interior.

The high vacuum main chamber (1) of the system is composed of stainless steel and bought from the Trinos Vakuum GmbH (now Pfeiffer Vacuum GmbH). The chamber is pumped by a Adixen (now Pfeiffer) Pascal 2015 C2 backing pump (2) and a Pfeiffer HiPace ${ }^{\circledR} 700$ turbopump (3). The pressure is measured by a Pfeiffer PKR 261 compact full range gauge (4) which is read out at the corresponding TPG 256 A Maxi Gauge (5). Three magnetron sources of type 2"ONYX from the Angstrom Sciences, Inc. and their corresponding shutter drives as well as a Modline ${ }^{\circledR} 5$ pyrometer (model 5R-1410000) from Ircon, Inc. are connected to the chamber from the right side via four flanges (6). The Aera Analog FC-R7700 CD flow controller (7) from Advanced Energy Industries, Inc. determines the Ar flux for each magnetron sources individually 


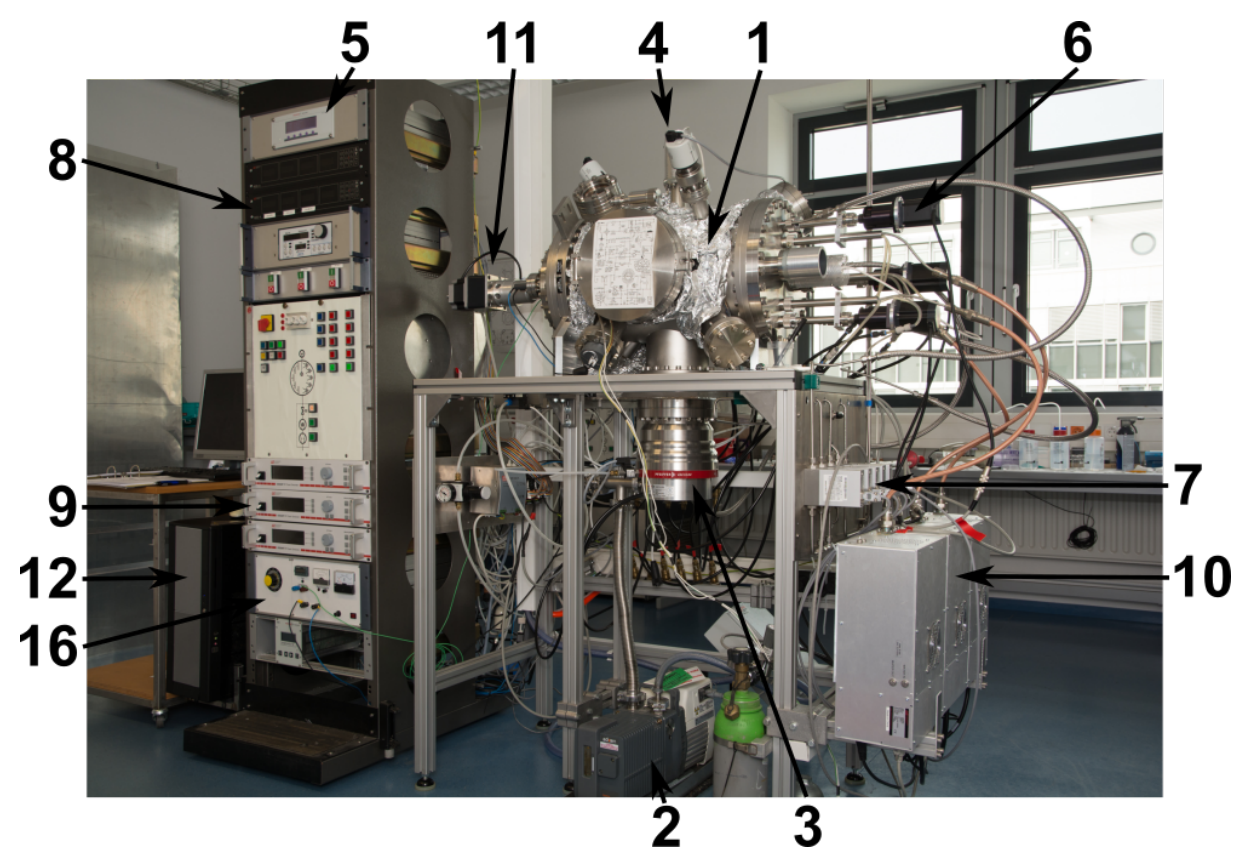

(a) Setup of the magnetron sputter system.

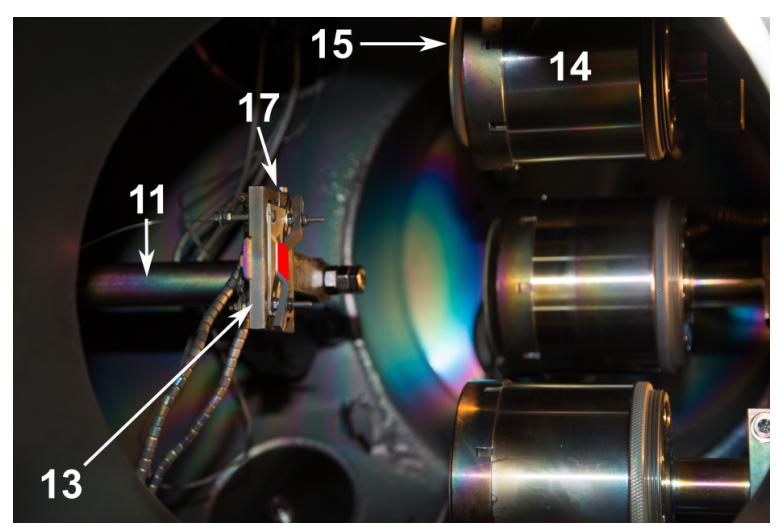

(b) Inside of the sputter chamber. The position of the sample is indicated in red.

Figure 3.1.: The magnetron sputter system that was used. The parts are denoted in the text above.

as well as the oxygen flux for the chamber and both are controlled via an Advanced Energy ROD-4 control unit (8). The magnetron sources are powered by Advanced Energy Cesar ${ }^{\circledR} 136$ (revision C) RF power generators (9) up to a power of $600 \mathrm{~W}$. The plasma in every source is stabilized by a corresponding Advanced Energy type VM1000A matching box (10) that keeps the plasma at $50 \Omega$ resistance by adapting two incorporated capacitances. Via a motorized drive (11), the PC control (12) can automatically positions the sample holder (13, with the sample indicated in red) in 
front of the chosen source (14). The PC control opens and closes the shutter (15) according to the defined sputter time. The sample holder has a BORALECTRIC ${ }^{\circledR}$ heating element from tectra $\mathrm{GmbH}$. With this, the sample can be heated up to $1273 \mathrm{~K}$. The maximum usable temperature depends on the adjusted oxygen partial pressure since the heater ages faster in the presence of $\mathrm{O}$. The temperature of the heating element is controlled via a pulsed automatic regulator (16) and is read-out is via a type $\mathrm{K}$ thermocouple (17) from THERMOCOAX (France). In addition, the surface temperature of the heating element and/or of the substrate can be measured precisely with the pyrometer, but only above $873 \mathrm{~K}$, and not during the deposition process.

\subsection{Transmission electron microscopy (TEM)}

Transmission electron microscopy (TEM) utilizes accelerated electrons that are transmitted through a sample. During transmission, some of the electrons interact with the sample yielding elastic and inelastic energy exchange. The different kinds of interaction can be measured with specialized detectors and used to form an image of the sample. A detailed introduction into TEM and corresponding acquisition techniques as well as spectroscopy is given by Williams and Carter [38].

Environmental TEM (ETEM) [96] enabled study of the $\mathrm{Pd} / \mathrm{r}-\mathrm{TiO}_{2}$ system in a hydrogen atmosphere. High resolution TEM (HRTEM) was applied to investigate the structure of the $\mathrm{Pd}$ and $\mathrm{r}-\mathrm{TiO}_{2}$ lattice as well as their matching on an atomic level. STEM in combination with (monochromated) EELS (probe size $\lesssim 5 \AA$ ) allowed for local mapping of the $\mathrm{Ti} L$ edges in $\mathrm{r}^{-\mathrm{TiO}_{2}}$ as a function of the interface distance $z$ and applied hydrogen pressure, see chap. 3.2.2. A thorough literature research has not discovered previous studies combining ETEM, STEM, and monochromated EELS. Such an application, as presented, would appear to be undocumented, therefore this work offers a new approach to local in situ interface investigations. The hydrogen interaction with the $\mathrm{Pd}$ films was studied by observing the dislocation density with high resolution STEM (HR-STEM), see chap. 3.2.3.

The $\mathrm{Pd}$ layers deposited on the pretreated $\mathrm{r}-\mathrm{TiO}_{2}$ crystals were prepared to make suitable TEM lamella. This was done by mechanical polishing and subsequent $\mathrm{Ar}^{+}$ion milling as described at section 3.2.1. The ETEM alignment was done in collaboration with V. Roddatis.

\subsubsection{Preparation of TEM lamellae}

Cross-section TEM lamellae were produced in collaboration with V. Roddatis following a procedure described by Süess et al. [97]. Two peaces were cut from a $\mathrm{Pd} / \mathrm{r}-\mathrm{TiO}_{2}$ sample. The Pd sides of both pieces were glued onto each other. Subsequently, the sample was thinned by mechanical polishing with a diamond lapping film. Next, the sample was thinned further with the use of low-angle $\left(6^{\circ}\right) \mathrm{Ar}^{+}$ion milling in a 
Gatan PIPS 691 at $3 \mathrm{kV}$ until perforation. Subsequently, the acceleration voltage was gradually decreased to prevent radiation damage or the formation of amorphous layers on the surfaces.

The TEM lamella configuration produced with this technique is shown in fig. 3.2. The lamella has a symmetric structure. On the left side, the $\mathrm{r}-\mathrm{TiO}_{2}$ crystal is visible

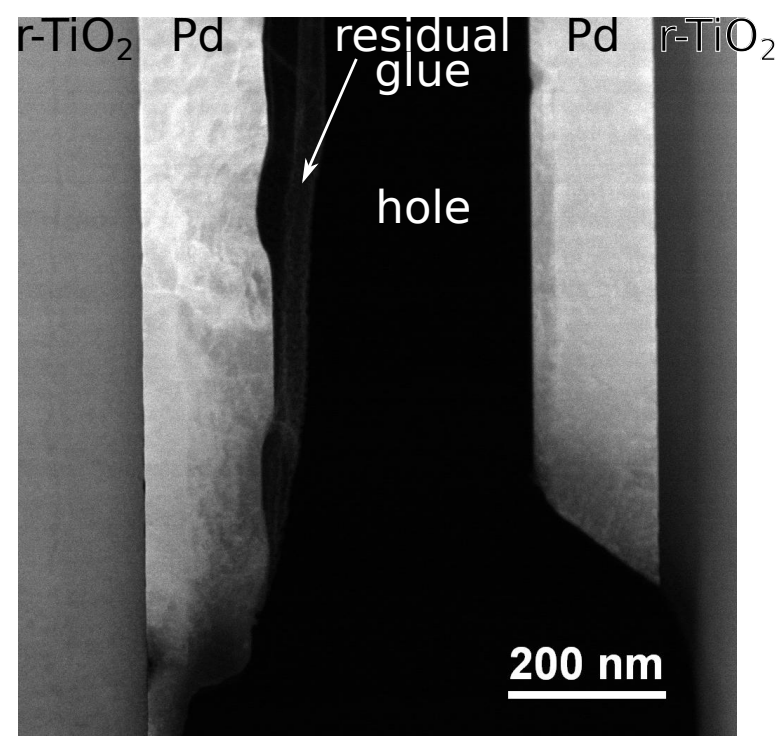

Figure 3.2.: High-angle annular darkfield (HAADF) image of a $\mathrm{Pd} / \mathrm{r}-\mathrm{TiO}_{2}$ lamella that was prepared following the procedure described in this chapter. The lamella exhibits a symmetric configuration $\left(\mathrm{r}-\mathrm{TiO}_{2} / \mathrm{Pd} /\right.$ hole/ $\left.\mathrm{Pd} / \mathrm{r}-\mathrm{TiO}_{2}\right)$. The $\mathrm{Pd}$ film thickness is approximately $200 \mathrm{~nm}$.

followed by the Pd layer to the right. On top of the Pd layer, remnants of the glue used are visible. The empty space is the mirror plane and a $\mathrm{Pd}$ layer on $\mathrm{r}-\mathrm{TiO}_{2}$ is therefore found going further to the right in the picture. The thickness of the $\mathrm{Pd}$ layer is approximately $200 \mathrm{~nm}$. The shown lamella was used for the in situ EELS experiments that are described in the next chapter.

\subsubsection{In situ EELS experimental procedure on $\mathrm{Pd} / \mathrm{r}-\mathrm{TiO}_{2}$}

To perform the in situ experiments, the prepared TEM lamellae were transferred to the ETEM. The ETEM is shown schematically in fig. 3.3. Subsequently, the ETEM was aligned to monochromated STEM, as described in appendix A.5. Next the environmental conditions were set up: the gas lines were pumped out up to the ETEM inlet valve and then the hydrogen bottle was opened. Hydrogen was present up to the gas inlet valve. Opening the ETEM inlet valve limits the minimum partial pressure to approximately $10^{-3} \mathrm{~Pa}$. However, dried air contains hydrogen with a volume fraction of approximately $500 \mathrm{ppb}$ [66] which is equivalent to a hydrogen partial pressure of $5 \cdot 10^{-2} \mathrm{~Pa}$. It can be assumed that the sample was in equilibrium with this hydrogen partial pressure before the experiment and that the background pressure is not limiting the experiments. The $\mathrm{Pd} / \mathrm{r}-\mathrm{TiO}_{2}$ system was studied for hydrogen pressures of $1 \mathrm{~Pa}$ and $10 \mathrm{~Pa}$. 
After the set-up for the desired pressure was achieved, the sample was equilibrated for $1 \mathrm{~h}$ with the column valves closed ('blanked' electron beam), preventing electrons from influencing the $\mathrm{H}_{2}$ loading. The important experimental part follows: On the optimized interfaces, rectangular spectrum imaging (SI) patterns were taken in situ at different places in the close vicinity of the $\mathrm{Pd} / \mathrm{TiO}_{2}$ interface in STEM mode.

A FEI Titan 80-300 ETEM was used and operated at $300 \mathrm{kV}$. The ETEM is equipped with a Cs-image corrector, a beam monochromator and a post-column GATAN image filter (GIF) Quantum 965. A sketch of the system is shown in fig. 3.3.

Figure 3.3.: Sketch of the used FEI Titan ETEM. Indicated with the blue area is the volume of the ETEM where the hydrogen is mainly present during the in situ experiments. The energy loss $\Delta E$ of the primary electrons (red arrow) at the sample (red bar) is measured in the bottom mounted EEL spectrometer. Reprinted and adapted with permission from Hansen et al. [98]. Copyright 2010 Taylor \& Francis.

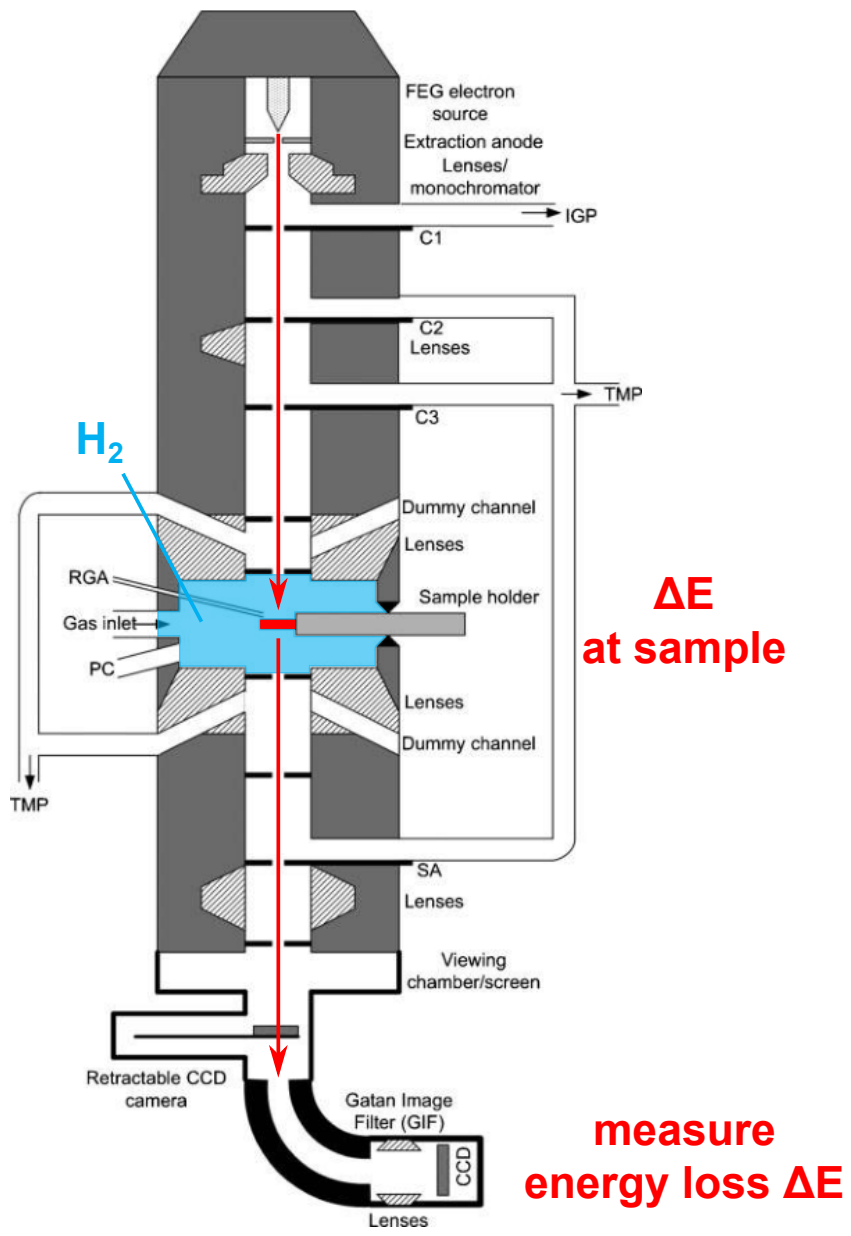

\subsubsection{In situ hydrogen loading experiments on Pd films}

A Pd film was investigated in the ETEM with high resolution STEM (HR-STEM) in collaboration with $\mathrm{V}$. Roddatis. The aim of these experiments was to study the dislocation density $\rho_{\mathrm{D}}$ as a function of the applied hydrogen gas pressure. Hydrogen pressures from $0.1 \mathrm{~Pa}$ to $500 \mathrm{~Pa}$ were applied on the $\mathrm{Pd} / \mathrm{r}-\mathrm{TiO}_{2}$ sample. The sample was prepared as described in chap. 3.2.1. 


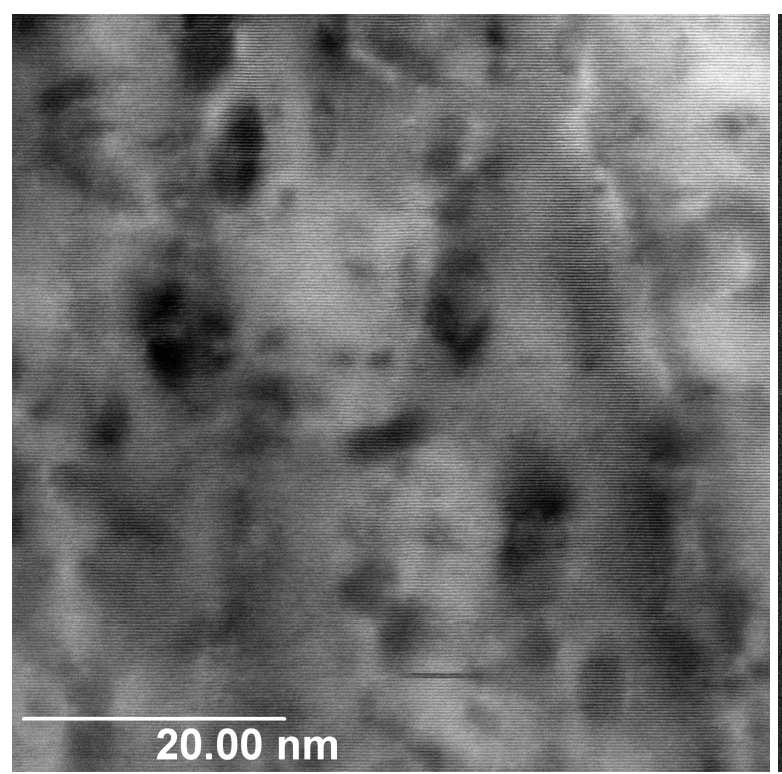

(a)

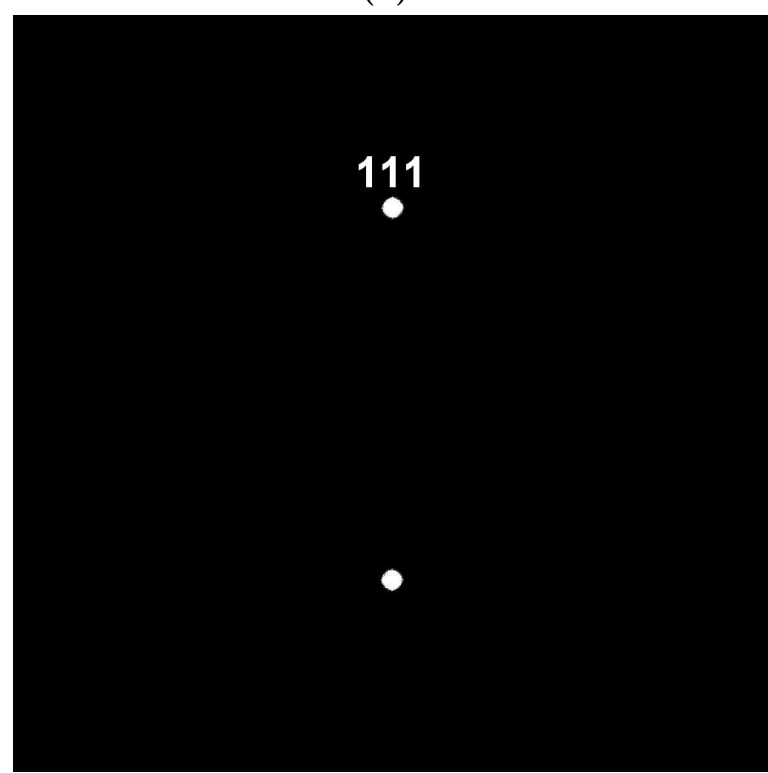

(c)

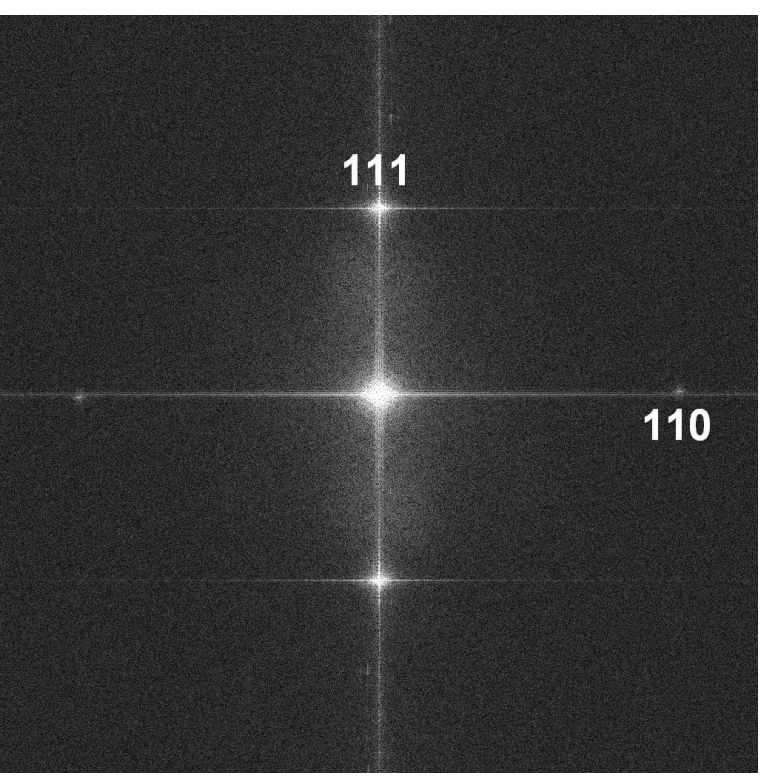

(b)

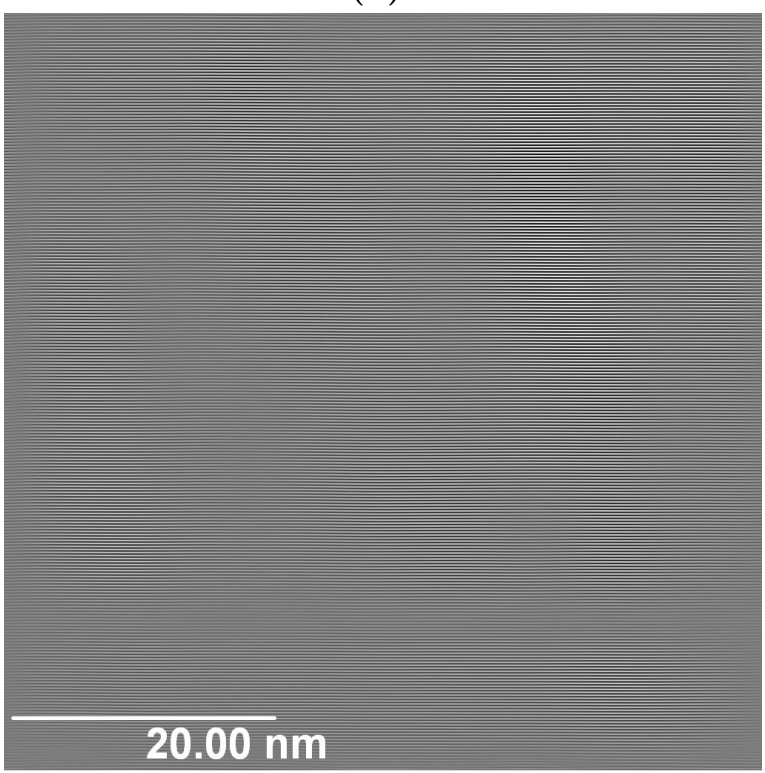

(d)

Figure 3.4.: Example of a high resolution STEM (HR-STEM) investigation to find dislocations in the $\mathrm{Pd}(111)$ plane. (a) HR-STEM image in the middle of a $\sim 200 \mathrm{~nm}$ Pd film deposited on a $\mathrm{r}-\mathrm{TiO}_{2}$ crystal. (b) shows the (reduced) FFT of the HR-STEM image. (c) a mask for the FFT back transformation was applied to select the $\mathrm{Pd}(111)$ reflexes in normal direction of the film. (d) the FFT back transformation of the original HR-STEM image. Only the $\operatorname{Pd}(111)$ planes are visible. On these images the number of dislocations was counted using an automatized script. 
The sample was equilibrated at the desired hydrogen pressure for $1 \mathrm{~h}$ in the ETEM without the presence of the electron beam. After equilibration, a HR-STEM investigation followed: A series of five HR-STEM images was taken in the middle of the $\mathrm{Pd}$ film at different positions for every applied hydrogen pressure.

From the HR-STEM images, the dislocation area density $\rho_{\mathrm{D}}^{(111)}$ was calculated in the $\mathrm{Pd}(111)$ planes in normal direction of the $\mathrm{Pd}$ film in collaboration with $\mathrm{V}$. Roddatis: In DigitalMicrograph, the fast Fourier transform (FFT) was calculated from the HR-STEM images and the (111) reflexes in normal direction of the Pd film were chosen with a mask. With the mask applied, a back transformation was performed yielding to an image containing just the $\operatorname{Pd}(111)$ planes in normal direction of the film. In the back transformed images, the number of dislocations was determined. An automatized script searched for intensity variations below a certain threshold which was found to be typical for the position of the dislocations and colored with a red line. Subsequently all possible dislocations were checked manually to confirm the individual dislocations. The dislocation density was calculated by dividing the number of confirmed dislocations by the total size of the investigated images.

The dislocation area density $\rho_{\mathrm{D}}^{(111)}$ was calculated via:

$$
\rho_{\mathrm{D}}^{(111)}=\frac{\sum_{i} \text { number of dislocations in image } i}{\sum_{i} \text { area of image } i}
$$

The evaluation process is shown in fig. 3.4. A HR-STEM image in the middle of the $\mathrm{Pd}$ film before hydrogen introduction is shown in fig. 3.4a. The image shown has a size of $3364 \mathrm{~nm}^{2}$. The calculated (reduced) FFT of the HR-STEM image is shown in fig. 3.4b. The FFT shows the $\mathrm{Pd}(111)$ reflexes at the top and bottom of the central spot. Other spots are not or nearly not visible due to the limited resolution in STEM mode. However, weak (110) spots appear on the left and right side. A mask is applied on the (111) reflexes in the FFT as shown in fig. 3.4c. From the masked region of the FFT, a back transformation is carried out. This back transformation is shown in fig. 3.4d. Here, just the $\operatorname{Pd}(111)$ planes in normal direction, which is the top direction in the image, are visible. On the back transformed images, the possible locations of the dislocations were marked with a red line automatically by a script in DigitalMicrograph. The red marked possible locations were verified manually and the confirmed dislocations were counted.

\subsection{Electron energy loss spectroscopy (EELS)}

In electron energy loss spectroscopy (EELS), the energy loss $\Delta E$ of the primary electrons, which have inelastically scattered due to interactions with the sample, is measured. This allows the investigation of plasmons ${ }^{2}$ as well as elemental specific edges.

${ }^{2}$ Plasmons are collective oscillations of the outer shell (or valence) electrons [39]. 
A brief introduction to EELS and the Ti $L$ edge is given in the theory in chap. 2.1.2 or in details by Egerton [39].

EELS was used to measure the $\mathrm{Ti} L_{3,2}$ edge from $\mathrm{r}-\mathrm{TiO}_{2}$ in the vicinity of the $\mathrm{Pd} / \mathrm{r}$ $\mathrm{TiO}_{2}$ interface at different hydrogen pressures. For the experiments, a methodology was developed to characterize small energy loss changes in EEL spectra. This was a necessary requirement: To successfully measure small energetic changes in the Ti $L$ ELNES a high energy stability as well as a high energy resolution was required. This was essential to resolve the $\mathrm{Ti} L$ edge with sufficient details to observe small energetic changes in it. The methodology that allowed this aim to be achieved is described in the following. The choice and reason for chosen acquisition parameters is given based on preliminary experiments in chap. 3.3.1. During this work some 10.000 single EEL spectra were evaluated. This was done meticulously and reliably with a evaluation procedure which is presented in chap. 3.3.2.

The developed methodology finally allowed an evaluation of physical parameters from the EEL spectra as a function of the distance to the interface $z$ and of the hydrogen pressure.

\subsubsection{Preceding EELS parameter refinement}

Preceding tests were necessary to determine the appropriate parameters for EEL spectra acquisition. This chapter explains which factors of the GIF can improve the EEL acquisition and how the corresponding parameter was chosen for the final EELS experiments. Additionally, features that occur in the microscope and can limit the experiments are discussed. A summary of the final parameters is given in chap. 3.3.2.

A short remark: The energy resolution of an EEL spectrometer is defined as the full width at half maximum (FWHM) of the focused zero loss peak (ZLP) [38].

\subsubsection{Monochromized zero loss peak (ZLP) description and energy resolution improvement}

A well aligned zero loss peak (ZLP) is crucial for EELS in general and, in particular, for measuring small effects with high energy resolution. This is especially important because the ZLP was subsequently used for the zero-loss centering and the deconvolution of the plasmon contribution to the core loss region ( $\mathrm{Ti} L$ edge).

It is important that the GIF is aligned (focused, minimized aberrations and astigmatism) in such a way that the ZLP is symmetric and maximized in intensity. The exposure time for the low loss region (that contains the ZLP) was chosen to be as high as possible ${ }^{3}$. The GIF alignment and a high exposure time are important for an

${ }^{3}$ One step before the display of the low loss spectrum turns yellow or red. This needs to be checked in the region of interest beforehand. 
accurate fit of the ZLP. DigitalMicrograph offers different models ${ }^{4}$ to fit the ZLP. The best accuracy was gained with the 'Reflected tail' model. The ZLP is appropriately fitted if every low loss spectrum is separated correctly into a 'Zero-loss Spectrum' and an 'Inelastic Spectrum' that has no (or hardly any) zero-loss contribution ${ }^{5}$. An example for a correct separation is shown in fig. 3.5. If the separation does not work, the

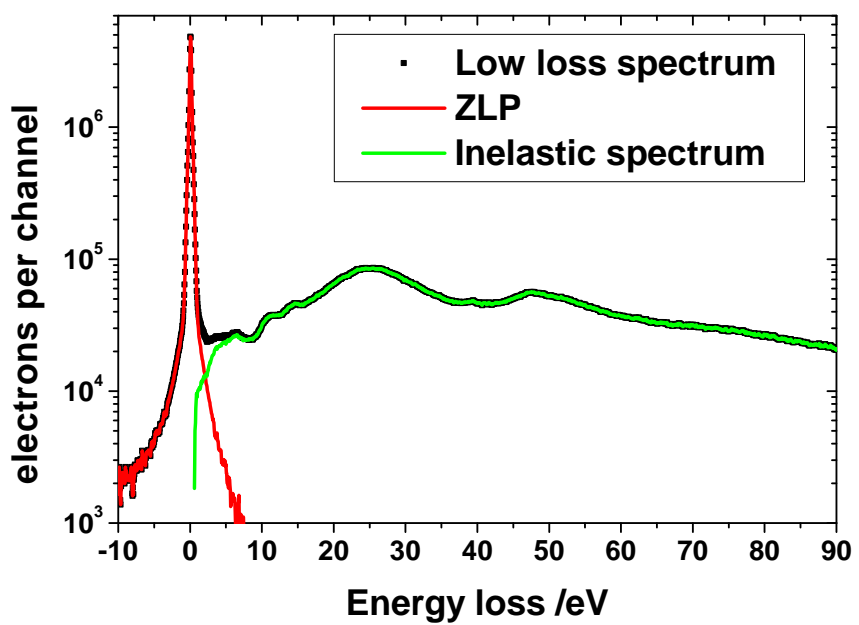

Figure 3.5.: A low loss spectrum (black) of a $\mathrm{r}-\mathrm{TiO}_{2}$ single crystal is shown. Fitting with the reflected tail algorithm works appropriately and results in a well separated ZLP (red) and inelastic spectrum (green). The energy resolution (or FWHM) obtained from the fit is approximately $350 \mathrm{meV}$.

signal-to-noise ratio will likely be bad and the ZLP needs to be aligned again with the GIF. The ZLP fit provides a calculated energy center and a FWHM that is a measure for the energy resolution. The acquired ZLP typically has its center not at $0 \mathrm{eV}$, but some $100 \mathrm{meV}$ off. With the calculated ZLP peak position, DigitalMicrograph can shift the ZLP back to ' $0 \mathrm{eV}$ '. This shift is also present in the core loss part of the spectrum, therefore the core loss spectrum was shifted as well. With the use of the monochromator, the energy resolution can in principle be tuned below $200 \mathrm{meV}$. But the high energy resolution is not stable for long. For the chosen EELS acquisition parameters, the energy resolution was kept stable below $500 \mathrm{meV}$. This condition was reached with a monochromator excitation of 1 and with the monochromator spot number 15 .

To further improve the energy resolution, the acceleration voltage of the primary electrons could be decreased, for example to $80 \mathrm{kV}$. An excellent example of this influence is shown in fig. 3.6. The energy resolution in EELS is shown against the exposure (or acquisition) time. It is shown that the energy resolution is reduced by reducing the

\footnotetext{
${ }^{4}$ Details about the models can be found in [99, EELS Analysis > EELS menu $>$ Zero-Loss > Preferences...].

${ }^{5}$ The inelastic spectrum should not be part of the zero-loss spectrum, nor should the ZLP be part of the inelastic spectrum.
} 
primary energy of the electrons from $200 \mathrm{keV}$ to $80 \mathrm{keV}$ for all exposure times. This argument is also applicable for $300 \mathrm{keV}$ electrons, as used. However, the lower energy of the primary electrons (e.g. $80 \mathrm{keV}$ ) increases the general scattering cross-section which yields to an increased intensity of the whole energy loss spectrum. Hence, a higher acceleration voltage (e.g. $300 \mathrm{kV}$ ) has the advantage that the signal-to-noise ratio is better. This is because the plural-scattering background decreases faster than the edge intensity with increasing acceleration voltage [38, chap. 39.2]. Thus, the $300 \mathrm{kV}$ alignment of the ETEM was used to get the highest signal-to-noise ratio for the Ti $L$ edge.

The GIF used has an entrance aperture that, in addition, limits the measured energy resolution of the GIF [38]. For the used GIF the following apertures can be chosen: no aperture, $5 \mathrm{~mm}$ and $2.5 \mathrm{~mm}$. While the larger aperture allows for higher signal, the smaller aperture gives better energy resolution. The entrance aperture defines the collection semi-angle which is typically in the range of 10 mrads [38]. The $2.5 \mathrm{~mm}$ aperture was used, as the energy resolution was more important than high intensity.

\subsubsection{Stabilization of energy stability, energy drift and spatial drift}

To detect the energetic changes at the interface requires the highest energy stability and the lowest energy and spatial sample drift. These topics are addressed in the following.

An important quantity was the energy resolution measured in the GIF. In a workshop [100], given by Prof. Kothleitner (from the FELMI-ZFE) in Göttingen, the dependency of the energy resolution as a function of the exposure time of the EEL spectrum was discussed. Fig. 3.6 shows that, with increasing exposure time, different contributions, which are indicated with the red arrows, decrease the energy resolution. For exposure times in the millisecond range, aberrations and high frequency noise limit the energy resolution. For exposure times up to seconds, low frequency noises add up, limiting the energy resolution. For exposure times above seconds, energy drift becomes a crucial limit for the energy resolution.

These influences are important when dual EELS is used to map the low and core loss spectrum at the same time. The low loss spectrum is typically measured with exposure times up to the millisecond range because the strong signal of the ZLP is limiting the exposure time. For the core loss part, including the Ti $L$ edges, exposure times up to seconds are typically used to get enough signal for the core edges. Thus, the core loss spectrum can have a worse energy resolution than the low loss spectrum and additionally contain energy drift, cf. fig. 3.6. To minimize these effects in the experiments, it was useful to bring the exposure time of the low and core loss spectrum as close together as possible and, in general, to measure 'as fast as possible'. This is addressed in more details later on in this section.

The energy drift of the whole microscope (from the monochromator down to the GIF) was estimated by measuring an EEL spectrum (summed up EEL line scan for 


\section{$\therefore$ FEI $\quad$ Resolution versus exposure time}
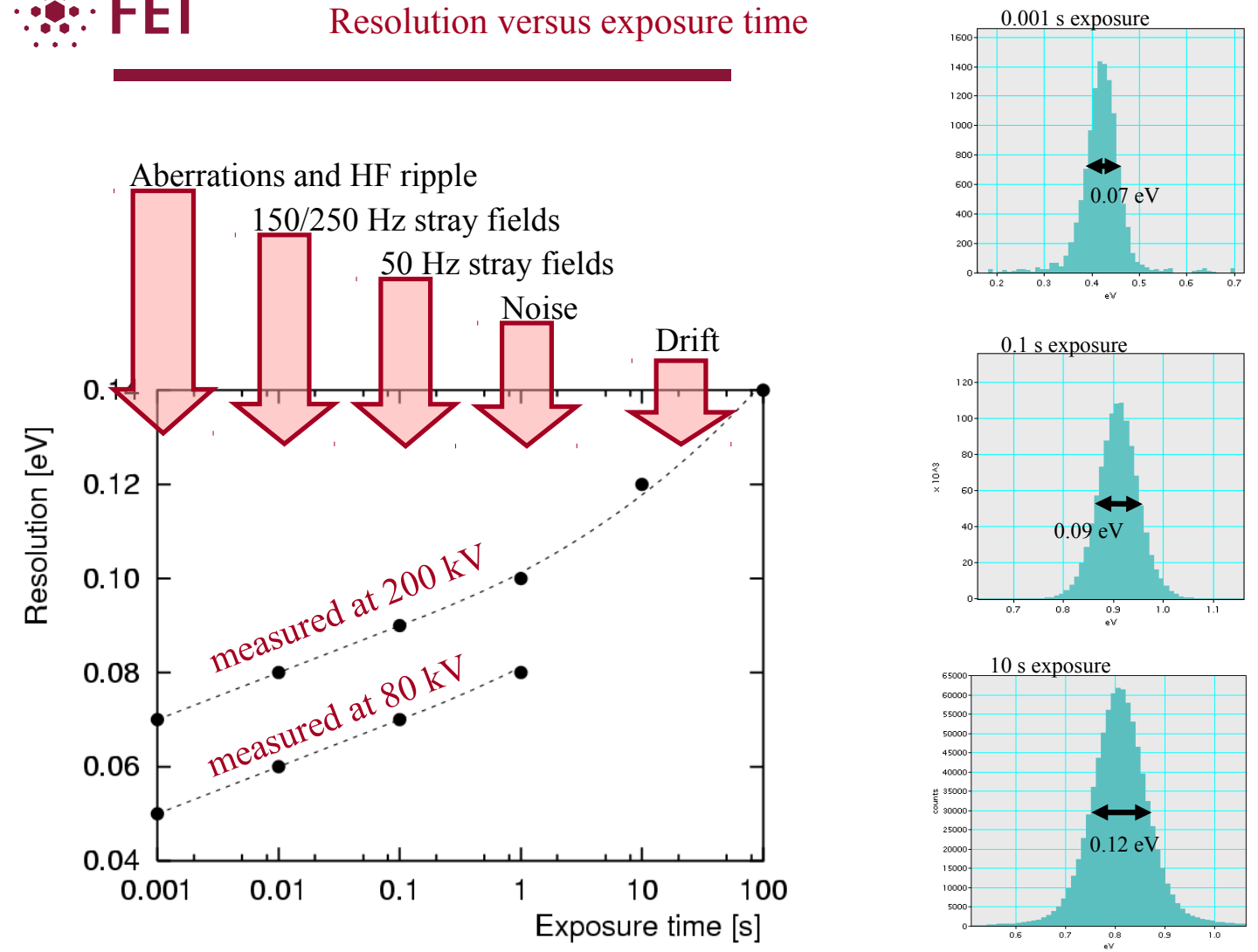

Figure 3.6.: Energy resolution in EELS as a function of the exposure time. The energy resolution depends on the used acceleration voltage, cf. $80 \mathrm{kV}$ vs $200 \mathrm{kV}$, of the primary electrons. Additionally, the energy resolution is limited by the exposure time since different noise adds up with increasing exposure time. The different noise contributions are indicated by the red arrows. ZLP examples are shown on the right side for different exposure times. With kind approval from Dr. Peter Tiemeijer (Thermo Fisher Scientific), cf. also [101].

better statistics) in a $\mathrm{r}-\mathrm{TiO}_{2}$ single crystal at the same position for different times under the same conditions. The position of the $\mathrm{Ti} L$ edge onset was evaluated as a function of the time when the spectrum was acquired as shown in fig. 3.7. Over time, the edge onset shifted to higher energies which was interpreted as an energy drift of the setup (ETEM + GIF). In this case, the drift was approximately $20 \mathrm{meV}$ in $25 \mathrm{~min}$ (including the fit uncertainties), cf. fig. 3.7, which is a rather low value. The acquisition time for a whole EEL SI pattern (cf. chap. 3.3.2) is on the order of 1 min, hence the contribution of the energy drift of the system can be assumed to be 
insignificant in the experiments ${ }^{6}$ performed.

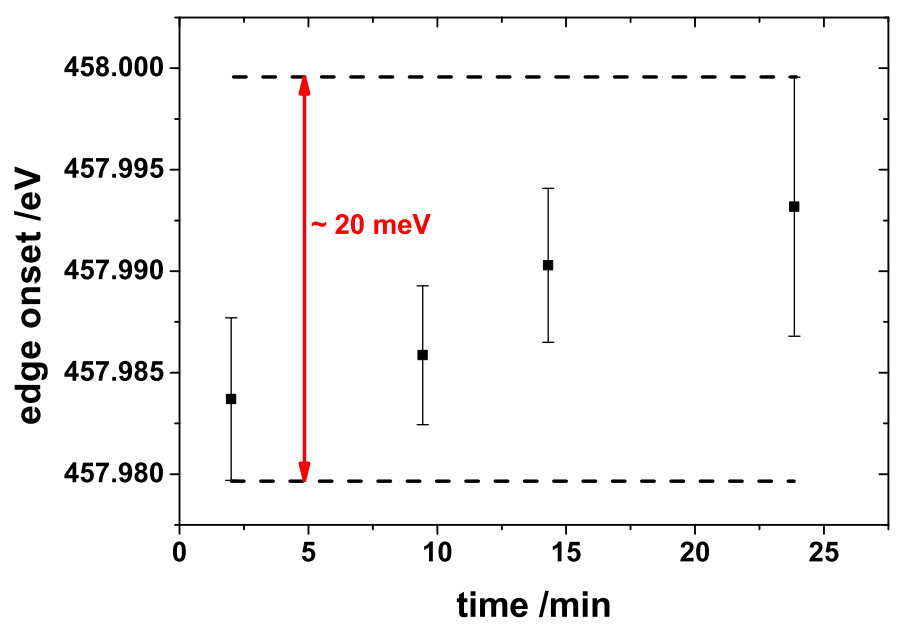

Figure 3.7.: The position of the $\mathrm{Ti} L$ edge onset of a $\mathrm{r}-\mathrm{TiO}_{2}$ single crystal is shown against the time when the EEL spectrum was acquired. No parameters or alignment was changed in between. The increase of the edge onset therefore corresponds to the energy drift of the whole microscope. The uncertainties shown represent the uncertainty of the corresponding fit (cf. chap. 3.3.2).

After the excitation of the monochromator and the alignment of the microscope's STEM mode, the ETEM typically exhibits drift (monochromator, other lenses, GIF). Therefore the system was left to stabilize for at least $6 \mathrm{~h}$, usually over night. Then the monochromator and STEM alignment was checked and adjusted again. After this iteration, the system was fairly stable, as is shown in fig. 3.7. This stabilization process was carried out before every experiment. Before the described procedure always a 'Full-Tune' of the GIF was carried out (in TEM mode) to minimize spectral aberrations.

Using dual EELS acquisition enables measurement of the low and core loss regions at the same time. Though, as described before, the exposure times of both regions can differ by orders of magnitude. The optimal condition would be the same exposure time for both loss regions: both loss spectra would contain the same noise and would have the same energy resolution. But since the core edges are not intense in comparison to the ZLP and the plasmons, this aim was unrealistic. It turned out that an exposure time of $10 \mathrm{~ms}$ to $50 \mathrm{~ms}$ for the core loss region and $0.2 \mathrm{~ms}$ for the low loss region was most suitable. Hence, these exposure times were used, together with the 'binning factor' of $130 \times$.

${ }^{6}$ In the performed EELS experiments, the alignment (monochromator, diffraction shift and GIF) was checked after each SI pattern. This ensured a well aligned system with respect to the discussed topic of energy drift. 
It could be shown that the ratio $t_{\text {core }} / t_{\text {low }}$ between the exposure time of the core $t_{\text {core }}$ and low loss $t_{\text {low }}$ region had a lower boundary for the used GIF. If the exposure times from core and low loss come too close, a contribution of the ZLP could be found in the core loss spectrum. This 'ghosting' phenomenon is known for the modern $300 \mathrm{kV}$ EELS detectors (Prof. Kothleitner [100]) ${ }^{7}$. An example of the 'ghosting' is shown in fig. 3.8. Here EEL spectra of the Ti $L$ pre-edge region for different ratios $t_{\text {core }} / t_{\text {low }}$ are shown. The closer the exposure times are the more prominent the broad feature

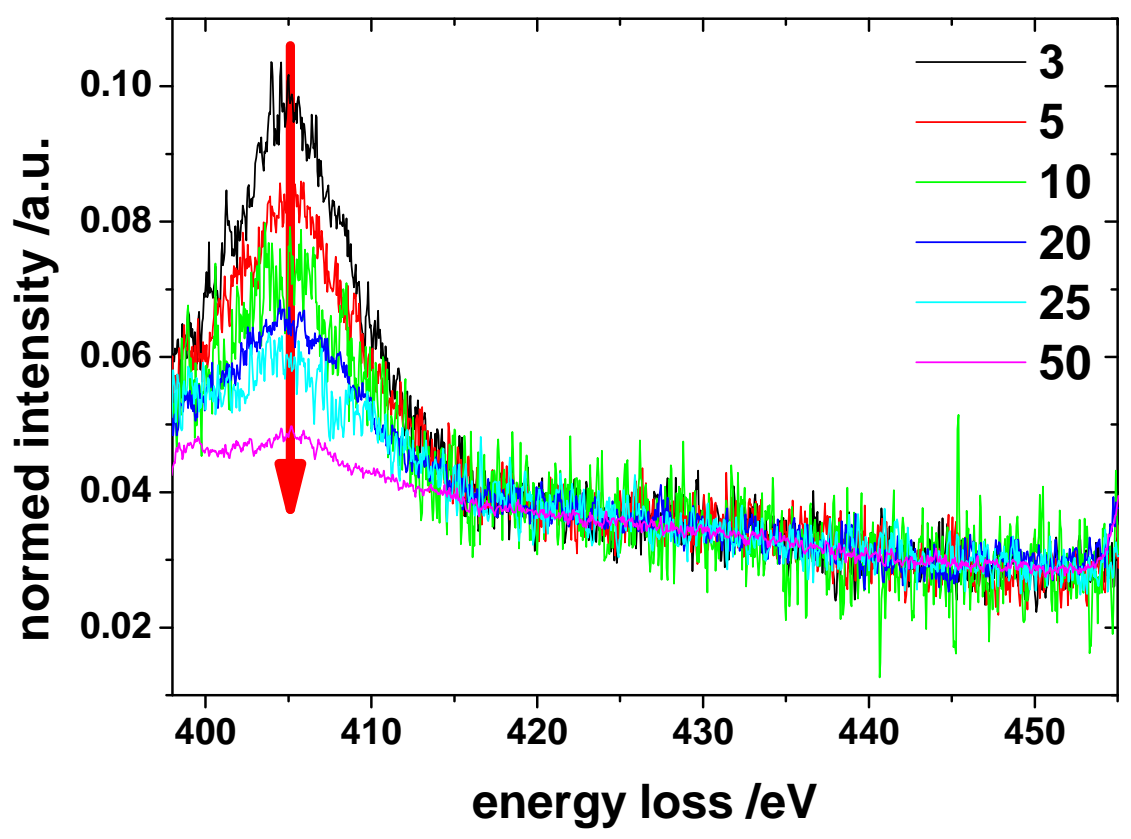

Figure 3.8.: Energy loss spectra of the Ti $L$ pre-edge region are shown for increasing exposure time ratios $t_{\text {core }} / t_{\text {low }}$ (color legend). The pre-edge region was normed to the plural scattering contribution between $420 \mathrm{eV}$ and $450 \mathrm{eV}$. Clearly, with increasing $t_{\text {core }} / t_{\text {low }}$ the 'ghosting' of the ZLP in the core loss spectrum decreases. At $t_{\text {core }} / t_{\text {low }}=$ 50 the 'ghosting' has all but vanished.

is in the lower energy loss part of the presented EEL spectra. This is the 'ghosting' of the ZLP and it decreases the possible fitting window for the pre-edge background for the $\mathrm{Ti} L$ edge evaluation: If the interval for the pre-edge background is too small, the fit of an inverse power law is not accurate. This yields a wrong estimate of the plural scattering contribution over which the Ti $L$ edge (above $455 \mathrm{eV}$ ) is laid on. Therefore the 'ghosting' has to be prevented by choosing a high enough exposure time ratio. Depending on the requirements for the corresponding experiment at the ETEM, $t_{\text {core }} / t_{\text {low }}$ has to be chosen appropriately. At $t_{\text {core }} / t_{\text {low }}=50$ the 'ghosting'

${ }^{7}$ Prof. Kothleitner: It can be circumvented by using 'older' $200 \mathrm{kV}$ detectors by sacrificing life time of these detectors if used with a $300 \mathrm{kV}$ electron gun. 
becomes insignificant. This can put a lower boundary on the exposure time for the core loss spectrum. As mentioned, typical ratios used were 100 to 500 since for lower ratios the $\mathrm{S} / \mathrm{N}$ ratio of the Ti $L$ edge was not high enough. With such 'high' values of $t_{\text {core }} / t_{\text {low }}$ no 'ghosting' of the ZLP was present.

Getting a good energy resolution with low drift influence is possible by measuring with low exposure times for the core loss region (cf. fig. 3.6), as discussed above. Though, the core loss region contains just a 'few' electrons and, therefore, the exposure time cannot be decreased arbitrarily, since the core loss spectrum will be limited by noise. The 'vertical' binning and the 'dark current correction' are two features of the GIF that can be, and were, used to improve the EEL spectra.

To lower the exposure time without sacrificing a lot of signal quality, the GIF is capable of 'vertical' binning: With binning, single detector channels are read out as one which reduces the read out time and thus increases the read out speed. Binning factors from $1 \times$ (no binning) to $130 \times$ (maximum binning) can be chosen for the installed GIF. The exposure time can therefore be reduced from the second range in the tenth of a millisecond second range. As compared to fig. 3.6, an decreasing exposure time results in an increasing energy resolution and reduces the influence of energy drift and low frequency noise to the acquired EEL spectra ${ }^{8}$. Decreasing the exposure time of the core loss spectrum eventually bring the EEL spectra to the noise limit of the GIF. The dominant noise results from small leakage currents, the so-called 'black noise' of the GIF's CCD. After every EEL spectra or SI pattern acquisition, the black noise ${ }^{9}$ can be measured and later be removed. This is done by acquiring a 'dark reference' after each EELS SI pattern which is also called 'dark current correction' (DCC). In DigitalMicrograph, the 'Improve Dark Reference' button acquires the dark reference and subtracts it automatically from the measured EEL spectrum or SI pattern. The influence of the DCC is shown in fig. 3.9. A sum of an EELS line scan with (red curve) and without (black curve) DCC is shown. Every EEL spectrum of the line scan is, with an exposure time of $50 \mathrm{~ms}$ for the core loss region, close to the noise limit. The DCC decreases the contribution of the black noise and thus increases the signal quality significantly. This results in a high quality summed Ti $L$ edge spectrum as shown with the red curve in fig. 3.9a. For intense features like the ZLP, the DCC has no influence as it can be seen in fig. 3.9b. However, the noise is reduced in other parts of the low loss spectrum. At the intensity level of the plasmons, the DCC reduces the noise: the DCC spectrum appears like a fit of the spectrum without DCC. In regions with low electron signal, the DCC is of significant advantage. This can be seen, for example, in fig. $3.9 \mathrm{~b}$, on the left tail of the ZLP or in the high energy part of the plural scattering contribution following the plasmons. It is recommended to always use DCC since it

${ }^{8}$ Paolo Longo from Gatan mentioned that binning can add an additional noise to EEL spectra. However, no significant influence was found and the binning factor was chosen as high as possible.

${ }^{9}$ Black noise is noise introduced to the GIF by (leakage) currents that flow even without any electron illumination. A dark reference measures this contributions and allows for correcting this noise from a taken EEL spectrum afterwards. 


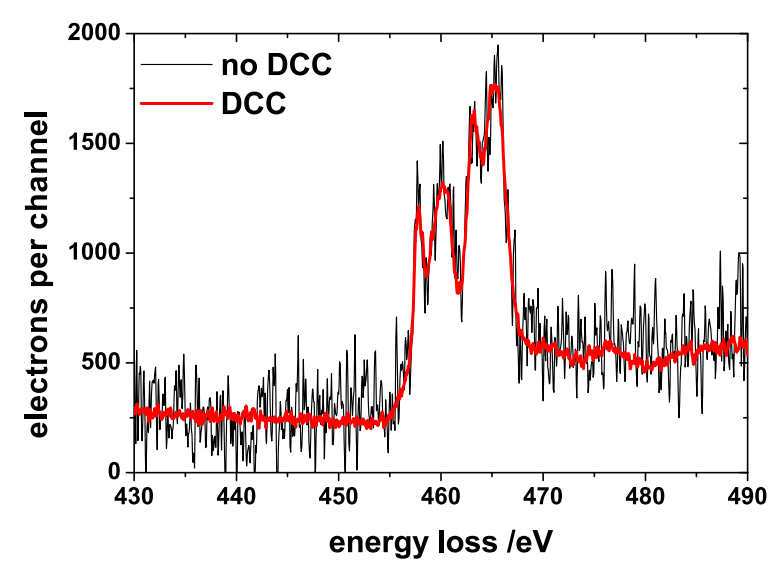

(a) Core loss spectrum.

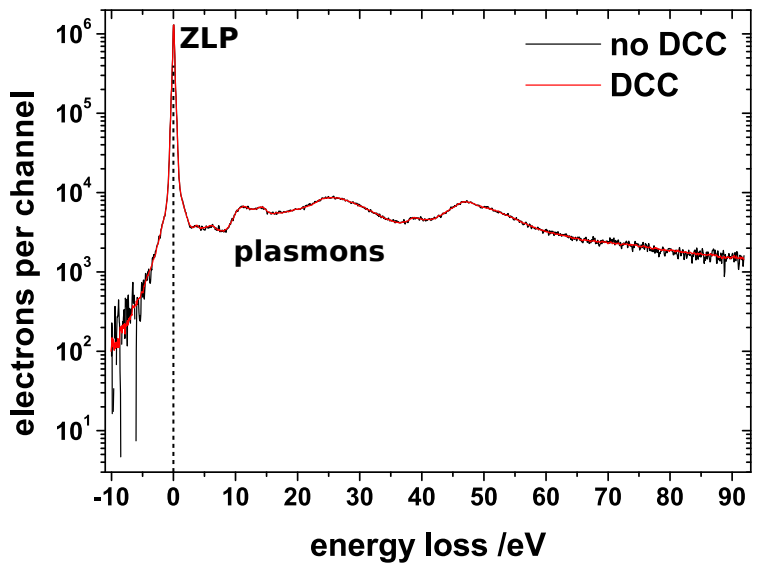

(b) Low loss spectrum.

Figure 3.9.: Energy loss spectra of a $\mathrm{r}-\mathrm{TiO}_{2}$ single crystal with (red) and without DCC (black). The spectra are summed up from the same line scan in which every EEL spectrum was acquired with $130 \times$ binning and an exposure time of $50 \mathrm{~ms}$. Each spectrum was close to the noise limit (black curves). The DCC improves the quality of each EEL spectrum (red curves) and yields to a significantly higher signal-to-noise ration (of the shown sum).

decreases the noise contribution to the EEL spectra significantly. In the 'worst' case of intense features, there is no influence of the DCC.

In addition to the energy drift, spatial drift of the TEM lamella also appears. This reduces the lateral resolution of the EEL spectra, especially close to the interface. Spatial drift can be minimized by reducing the total acquisition time of the SI pattern and by correcting with the 'spatial drift correction' of the microscope. Total collection times of the SI pattern between $60 \mathrm{~s}$ and $180 \mathrm{~s}$ provided the best compromise between enough signal (which is important for the subsequent binning discussed in chap. 3.3.2) and low energy drift influence (as discussed above).

In addition, a spatial drift correction of the TEM was used as shown in fig. 3.10. The correction could be used even after each acquired EEL spectrum or after each line of the EELS pattern. Though, this would result in high total acquisition times of the whole pattern. A good compromise was found by using the spatial drift correction after every four lines of the SI pattern. The spatial drift was of significant importance since the EEL spectra are compared afterwards as a function to the interface distance: if the interface moves with respect to the SI pattern, a change in the single EEL spectra can be reduced or even averaged out by the subsequent binning.

In summary, the following conditions were chosen for the EELS experiments as optimal parameters followed from the above discussion: DCC in combination with $130 \times$ binning and exposure times on the order of $0.1 \mathrm{~ms}$, for the low loss region, and between $10 \mathrm{~ms}$ and $50 \mathrm{~ms}$, for the core loss region, of the Ti $L$ edge revealed the best 
results.

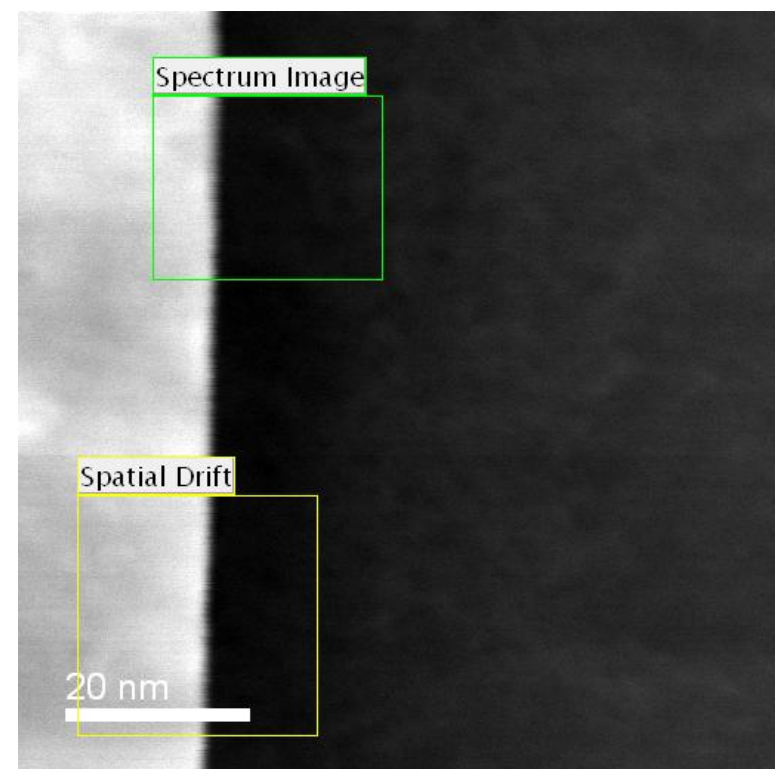

Figure 3.10.: HAADF image with monochromated STEM of the $\mathrm{Pd}$ (light) $/ \mathrm{r}-\mathrm{TiO}_{2}$ (dark) interface region. Indicated is an acquired EEL SI pattern as well as the window used for the spatial drift correction.

Minimized electrostatic charging influence and beam damage Long electron illumination of the sample can yield to electron beam induced electrostatic charging and atomic displacement [46] (cf. chap. 2.1.2.1), hence measuring 'as fast as possible' has a further advantage: the electron dose (per pixel) is as low as possible. If the step size is of the same order as the beam diameter, each location of the sample is illuminated just once per SI pattern. Thereby, the influence of electron beam induced electrostatic charging and atomic displacement was decreased to a minimum. Thus, 'subpixel scanning' features were not used for the acquired SI patterns.

The electron dose that was present during the illumination of the sample with the electron beam can be estimated: Screen currents and exposure times per pixel were approximately $130 \mathrm{pA}$ and $0.05 \mathrm{~s}$, respectively. The beam diameter was approximately $0.5 \mathrm{~nm}$. That implies a electron fluence (/dose) of approximately $3300 \mathrm{C} / \mathrm{cm}^{2}(\widehat{=} 2.07$. $\left.10^{6} \mathrm{e}^{-} / \AA^{2}\right)$.

\subsubsection{Pre-edge background and spectrometer dispersion impact}

The Ti $L_{3,2}$ edge lies on a pre-edge (or plural scattering) background. This background needs to be described correctly to do a proper evaluation of the Ti $L$ ELNES.

The pre-edge background is typically fitted via an inverse power law function [38, chap. 39.4.B]:

$$
I=A \cdot(\Delta E)^{-r},
$$

here $I$ is the intensity in the channel of energy loss $\Delta E, A$ is a prefactor and $r$ is a constant that typically ranges between 2 and 5 . The fitting window for the power 
law is defined by a low and a high energy side. Its size should be between $10 \%$ to $30 \%$ of the edge onset value and its high energy side should end $5 \mathrm{eV}$ before the edge onset (of the corresponding energy loss edge), cf. [38, chap. 39.4.B] and [100, EELS Quantification, p. 14]. Thus, a window between $407 \mathrm{eV}$ and $452 \mathrm{eV}$ was used for the fitting, since the $\mathrm{Ti} L_{3}$ edge onset is typically at around $458 \mathrm{eV}$. The low energy side of the window $(407 \mathrm{eV})$ could, in principle, be chosen to be smaller, which would increase the fit interval for the pre-edge background. But for the samples used, the appearance of the $\mathrm{Pd} M(>335 \mathrm{eV})$ edge in the interface vicinity can then lead to incorrect fits of the pre-edge background.

The GIF spectrometer dispersion determines how the collected electrons are binned in energy and finally are displayed in the EEL spectrum. The dispersion can be chosen between $1 \mathrm{eV} / \mathrm{ch}$ and $0.01 \mathrm{eV} / \mathrm{ch}$. Large dispersion values are typically used for overviews in the core loss region as they do not allow for high energy resolution. For fine details, dispersion values of $0.1 \mathrm{eV}$ and below are common. The GIF used has 2000 channels (ch), meaning the smallest dispersion results in an energy loss window of $20 \mathrm{eV}$. This is insufficient to collect the complete $\mathrm{Ti} L_{3,2}$ edge and enough pre-edge background. Dispersions of $0.05 \mathrm{eV} / \mathrm{ch}$ and $0.1 \mathrm{eV} /$ ch were used. The former allows for better energy resolution and the latter allows for recording the $\mathrm{O} K$ edge $(\gtrsim 532 \mathrm{eV})$ in addition to the Ti $L$ edge.

\subsubsection{Optimization of the interface alignment}

The alignment of the interface was an important issue. If the interface and the electron beam are not parallel, EELS will sum up information from different interface distances over the lamella depth (in the beam direction). The TEM lamella were typically aligned via a pole of the $\mathrm{r}-\mathrm{TiO}_{2}$ crystals, an example is shown in fig. 3.11a.

A tilting experiment was performed to confirm that the alignment using the $\mathrm{r}-\mathrm{TiO}_{2}$ pole also aligns the interface as parallel as possible to the electron beam. The lamella was tilted out of the aligned position by approximately $18^{\circ}$; this situation is shown in fig. 3.11b. While tilting, two lines were moving from the interface to both sides as indicated with the white arrow. These were interpreted as the top and bottom part of the interface that were not parallel to the electron beam any more. This experiment confirmed that the initial alignment is the 'sharpest' without lines. Hence, the interface was aligned satisfactorily parallel to the interface. That this was the case. is not astonishing, the $\mathrm{r}-\mathrm{TiO}_{2}$ crystals had a very flat surface due to their small miscut (cf. chap. 4.1.1). Thus, the $\mathrm{Pd} / \mathrm{r}-\mathrm{TiO}_{2}$ samples and their interfaces were aligned by a suitable pole of the $\mathrm{r}-\mathrm{TiO}_{2}$ crystal.

\subsubsection{Influences of carbon (C) contamination and cold trap}

Carbon (C) contamination can be a severe problem in EELS for edges that follow the $\mathrm{C}$ $K$ edge which starts at an energy loss of approximately $284 \mathrm{eV}$. The $\mathrm{C}$ cross-section can 


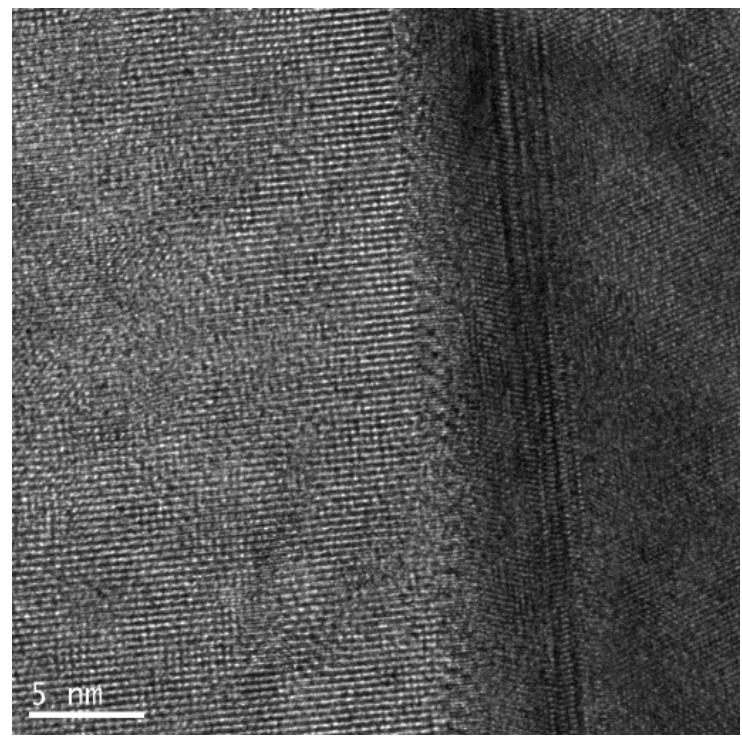

(a) Interface aligned via $\mathrm{r}-\mathrm{TiO}_{2}$ crystal.

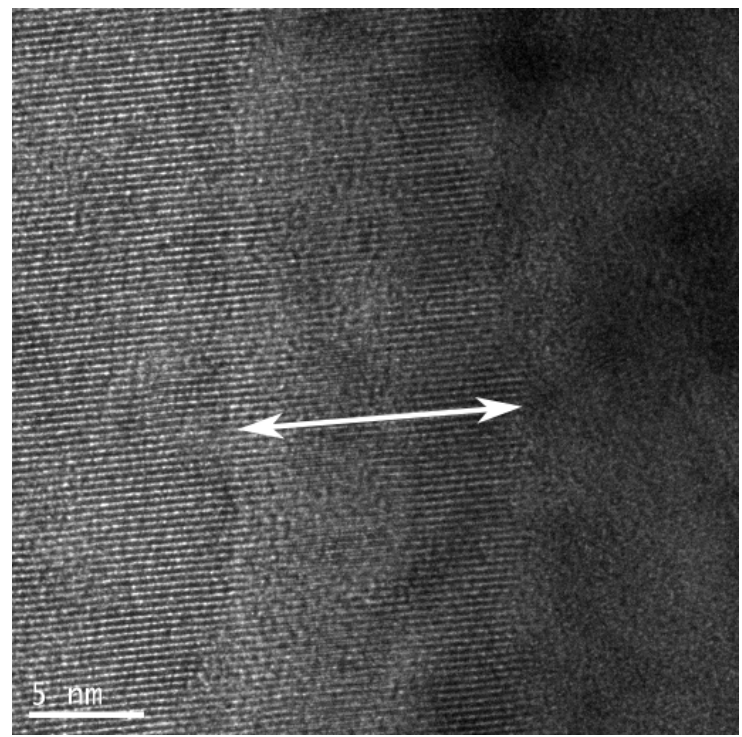

(b) Similar place, sample was tilted approximately $18^{\circ}$.

Figure 3.11.: High resolution TEM (HRTEM) picture of the $\mathrm{Pd} / \mathrm{r}-\mathrm{TiO}_{2}$ interface region. The bright part represents the $\mathrm{r}-\mathrm{TiO}_{2}$ (left) and the darker part the $\mathrm{Pd}$ (right) side. Tilting the interface out of the aligned position (3.11a) revealed two lines, which are indicated by the double headed arrow, moving from the initial interface position to the sides (3.11b). The interface shown had a roughness between $3 \mathrm{~nm}$ and $5 \mathrm{~nm}$ as the $\mathrm{r}-\mathrm{TiO}_{2}$ crystal was not pretreated.

have a long tail to higher energy losses which increases the plural scattering background significantly. The influence of $\mathrm{C}$ contamination on the Ti $L$ edge spectra is shown in fig. 3.12 for a sample that was directly transferred to the ETEM after its preparation. Here, two EEL spectra in the $\mathrm{r}-\mathrm{TiO}_{2}$ single crystal part are shown. One was acquired without additional hydrogen in the ETEM column (black) and the other one was acquired at $1 \mathrm{~Pa}$ hydrogen (red). A low jump ratio ${ }^{10}$ of 2.6 was obtained indicating a high contribution of $\mathrm{C}$ species to the $\mathrm{Ti} L$ pre-edge background. It turned out that the introduction of small hydrogen pressures in the ETEM significantly reduced the $\mathrm{C}$ contribution as shown with the red EEL spectrum in fig. 3.12. The jump ratio increased to 4.3, which is a significant improvement. Hydrocarbon species are typically discussed as the origin of carbon contamination in the TEM [46]. As may be imagined hydrogen can flush away the hydrocarbon molecules and thus reduce a deposition of C species. However, the origin of this effect remains unclear.

A cold trap, that is typically cooled with liquid nitrogen, can be used to improve

${ }^{10}$ The jump ratio is defined as "the ratio of the maximum edge intensity $\left(I_{\max }\right)$ to the minimum intensity $\left(I_{\min }\right)$ in the channel preceding the edge onset" [38]. 


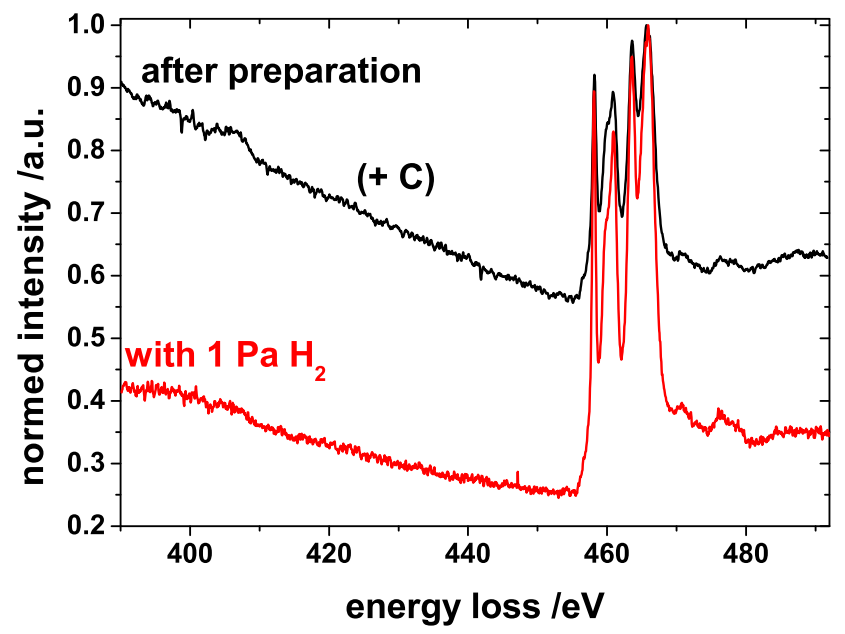

Figure 3.12.: Energy loss spectrum of a $\mathrm{r}-\mathrm{TiO}_{2}$ single crystal. The EEL spectra were normed to the maximum of the $d$ white line. The Ti $L$ edge and the corresponding preedge background are shown for the lamella after the transfer into the ETEM (black) and after the additional introduction of $1 \mathrm{~Pa}$ hydrogen (red). In the as prepared state $\mathrm{C}$ contributes strongly to the $\mathrm{Ti} L$ pre-edge region. The $\mathrm{C}$ contribution is significantly reduced in $\mathrm{H}$, the jump ratio increased from 2.6 to 4.3 .

the background pressure of a TEM by absorbing residual gases (e.g. water). In an ETEM, a gas of choice is leaked in the TEM column. This additional gas can contain additional residual gases. The cold trap can be used to reduce these unwanted residual gases as well. Therefore it can be of advantage to use the cold trap in ETEM mode as it was done for the performed EELS experiments.

To investigate a possible influence of the cold trap on the sample, the temperature at the TEM holder was measured for different hydrogen pressures, which is shown in fig. 3.13. The holder temperature decreased from $22^{\circ} \mathrm{C}$ at $10^{-5} \mathrm{~Pa}$ to $12^{\circ} \mathrm{C}$ at $10 \mathrm{~Pa}$ hydrogen and increased to $16^{\circ} \mathrm{C}$ at $100 \mathrm{~Pa}$ of hydrogen, cf. fig. 3.13. It seems that the hydrogen introduced a thermal connection between the cold trap and the TEM holder/lamella at least at $10 \mathrm{~Pa}$. The increasing temperature at $100 \mathrm{~Pa}$ hydrogen can hint on a stronger flow of the hydrogen that transports out some of the hydrogen that has thermally interacted with the cold trap. In addition, it was found that the cold trap becomes inefficient to use at higher hydrogen pressures: The liquid nitrogen evaporated entirely after a maximum of $30 \mathrm{~min}$, which is rapid, at $100 \mathrm{~Pa}$ hydrogen. This can also explain the strong spatial drift of the TEM lamella/holder occurring at $100 \mathrm{~Pa}$ hydrogen. This made the EELS experiments unreliable at this pressure. An additional influence to the observed temperature changes could be the interaction of hydrogen with the TEM holder itself, such as by changing its resistance. It is worth studying the interaction of the gas environment with the TEM sample holder in the future, to determine whether is it through a thermal connection or through a direct 


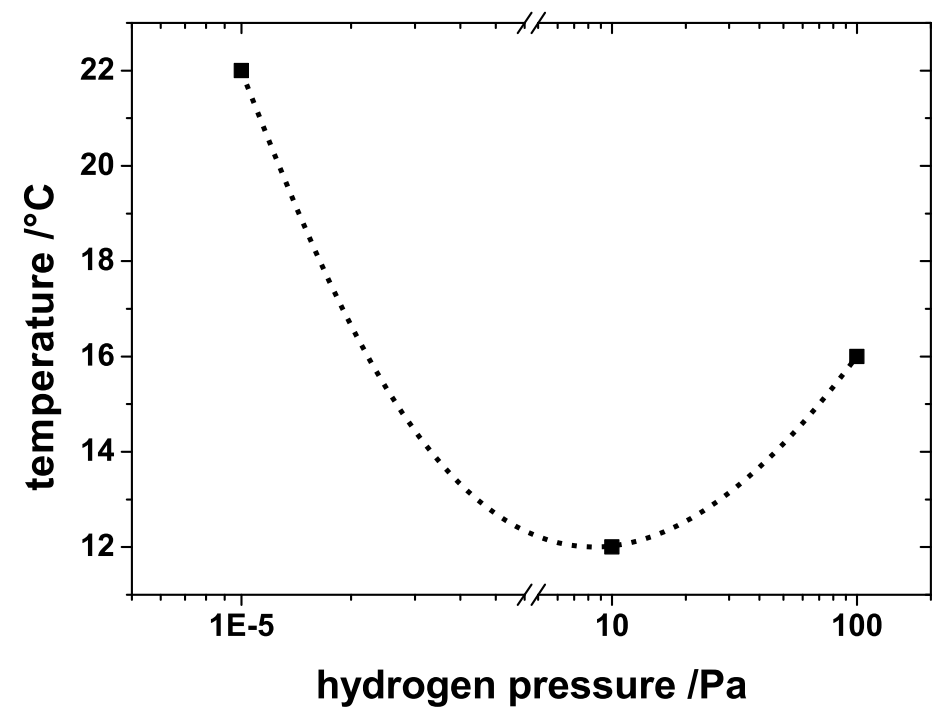

Figure 3.13.: The temperature of the TEM holder is shown against the hydrogen partial pressure in the ETEM. The cold trap was used in the experiment. The chosen hydrogen pressure influences the temperature at the holder. This suggests a thermal coupling between TEM holder and cold trap. The dotted black line is added highlight the trend.

gas interaction.

The cold trap was used for the experiments performed. For future experiments, though, it is recommended to not use the cold trap together with a hydrogen gas environment, in the ETEM.

\subsubsection{Developed advanced EELS methodology}

The EELS methodology is separated into two parts. In chap. 3.3.2.1, the acquisition procedure and the corresponding acquisition parameters of the EEL spectra are summarized. In chap. 3.3.2.2, the data analysis including the evaluation procedure is explained in detail. For the latter the software DigitalMicrograph ${ }^{\circledR}$ (version 2.32.888.0) from the Gatan, Inc. and MATLAB ${ }^{\circledR}$ (R2014a, v8.3.0.532) from The MathWorks, Inc. were used.

\subsubsection{Spectra acquisition}

To get information about changes in the $\mathrm{Ti} L$ edge in $\mathrm{r}-\mathrm{TiO}_{2}$ close to the $\mathrm{Pd} / \mathrm{r}-\mathrm{TiO}_{2}$ interface, a rectangular spectrum imaging (SI) pattern was defined and aligned parallel to the interface as schematically drawn in fig. 3.14. The SI pattern defines a frame in which EEL spectra were acquired as indicated with the red dots. The acquisition started on the right bottom side as indicated with the green circle. It continued in 


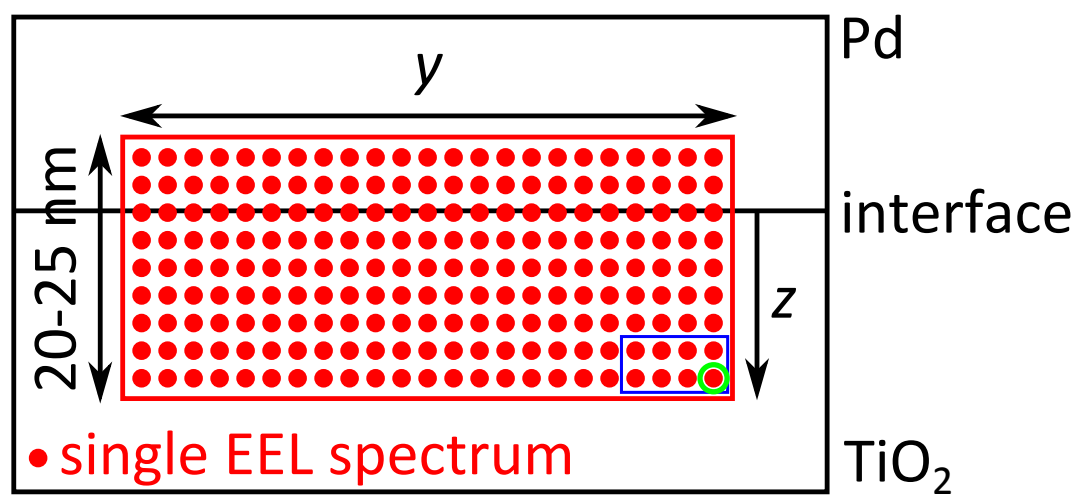

Figure 3.14.: Sketch of a rectangular EELS spectrum imaging (SI) pattern that is representative for this work. The sketched SI pattern aligned parallel to the $\mathrm{Pd} / \mathrm{r}$ $\mathrm{TiO}_{2}$ interface. It is the sum of single EEL spectra acquired at the positions indicated with the red dots. Therefore, their amount is depending on the SI pattern size and the chosen step size in it. The interface distance is defined as $z$. The green circle is the starting point of the measurement. The electron beam moves orthogonal to the interface $(-z$ direction) and then continues with the next row to the left ( $y$ direction). After the measurement the single EEL spectra were binned (blue box) to receive the most acceptable signal-to-noise ratio.

the $-z$ direction, after the first row was completed the next column to the left was acquired and so on. Hence, the EEL spectrum that was acquired last is left side top. The blue frame indicates a subsequent EELS spectrum binning that improved the signal-to-noise ratio. The binning was mainly in $y$ direction since in $z$ direction changes of the Ti $L$ edge were expected.

Based on the preceding experiments (see chap. 3.3.1) appropriate parameters were chosen for the EELS SI pattern acquisition which are summarized in appendix A.5. In one SI pattern a couple of 100 to approximately 1000 single EEL spectra were acquired, depending on the chosen size of the SI. During the experiments several ten of thousands single EEL spectra were evaluated. This required a partially automatized evaluation which was carried out in two steps: first, in DigitalMicrograph, the EEL data were preliminarily treated and, second, the data was analyzed with a self written script in MATLAB. The details are described in the next section.

\subsubsection{Evaluation procedure and automatization}

The collected rectangular SI pattern consisted of many single EEL spectra. The following procedure was applied on every pattern to calculate physical parameters out of the Ti $L$ ELNES as a function of distance to the interface $z$ and of the hydrogen pressure. Corresponding experimental uncertainties were calculated.

In DigitalMicrograph, the following steps were applied to correct for energy shifts, 
the pre-edge background, and the plasmon contribution to the Ti $L_{3,2}$ edge:

- Zero-loss centering using reflected tail: The ZLP was fitted via the 'Reflected tail' method in DigitalMicrograph via 'Extract Zero-Loss'. Using the 'Correct Zero-Loss Centering' function of DigitalMicrograph both the low loss and core loss spectrum were corrected for the calculated offset from the ZLP fit.

- Spatial binning: The individual EEL spectra in the SI pattern were partially binned to achieve an appropriate signal-to-noise ratio. The binning is indicated with the blue box in fig. 3.14. Changes were expected as a function of $z$ and therefore binning was minimal in $z$ direction: typically two steps were binned in $z$. For a step size of $0.5 \mathrm{~nm}$ this increases the final step size to $1 \mathrm{~nm}$. The $z$ positions after binning are written as $(0.5 \pm 0.5) \mathrm{nm},(1.5 \pm 0.5) \mathrm{nm}$, and so on. Thus, depending on the amount of binning in $z$ direction non-integer step sizes can occur. The binning was done by a script in DigitalMicrograph.

- Pre-edge background subtraction: The pre-edge background was subtracted by fitting an inverse power law function $\left(I=A \cdot(\Delta E)^{-r}\right)$ with a window for fitting between $407 \mathrm{eV}$ and $452 \mathrm{eV}$ to the $\mathrm{Ti} L$ edge. The details of pre-edge background treatment are described in detail by Williams [38, chap. 39.4.B]. An example of a fitted power law and a pre-edge subtracted $\mathrm{Ti} L_{3,2}$ edge is shown in fig. 3.15. Here, the pre-edge background of a collected EEL spectrum (black) was fitted with an inverse power law (green) and subtracted (red).

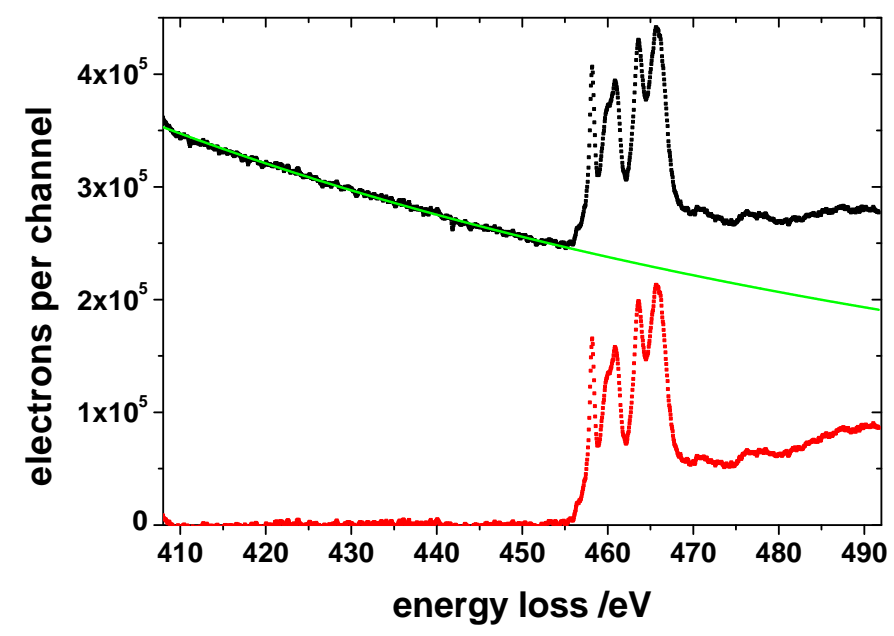

Figure 3.15.: Energy loss spectrum of the Ti $L_{3,2}$ edge taken from a r- $\mathrm{TiO}_{2}$ single crystal (before hydrogen loading). Shown is the acquired EEL spectrum (black), the fitted pre-edge background using an inverse power law function (green) and the EEL spectrum after the pre-edge background subtraction (red). 
- Deconvolution of the plasmon contribution: The plasmon (or mixed scattering) contribution was removed from the $\mathrm{Ti} L_{3,2}$ edge by the Fourier-ratio method, which is a standard procedure $[38,43,100]$. For the deconvolution, the following 'Fourier Deconvolution Preferences' were used DigitalMicrograph: 'Reflected tail' and 'Reconvolution method: zero-loss modifier'11. It is important that every core/high loss spectrum is deconvoluted with its corresponding low loss spectrum. The better the fit of the ZLP with the chosen model, the less noise is induced by the deconvolution. Typically (by using the reflected tail model), the noise contribution was small and the noise in the deconvoluted spectrum was dominated by the noise from the measurement. The deconvolution removes the electrons that have scattered more than once from the $L_{3,2}$ edge, typically this means a plasmon and a Ti ion were excited. To remove the plural scattering contribution, the plasmon signal of the low loss region was deconvolved from the core loss spectrum containing the $\mathrm{Ti} L_{3,2}$ edge. The deconvolution mainly influenced the cross-section for energy losses higher than $467.5 \mathrm{eV}$, which is higher than the $\mathrm{Ti} L_{2}$ edge. This is shown in a comparison of a $\mathrm{Ti} L_{3,2}$ edge spectrum before and after the deconvolution in fig. 3.16a. Subsequently, a Hartree-Slater

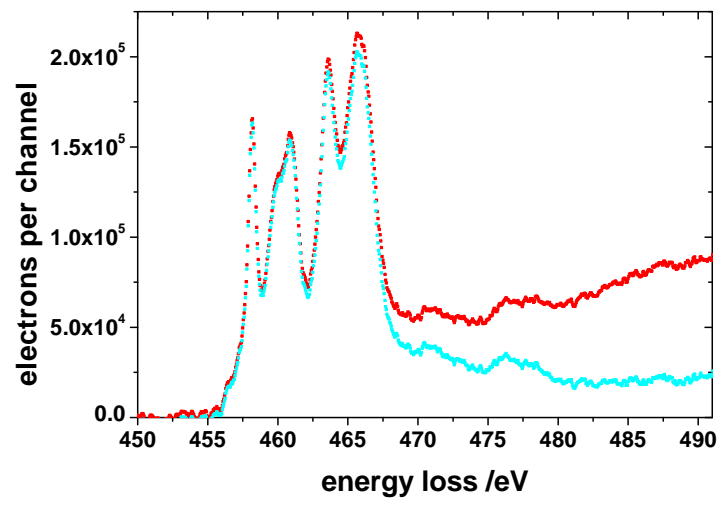

(a) Energy loss spectrum after the pre-edge background subtraction from fig. 3.15 (red). The deconvolved spectrum (Fourier-ratio method) is represented in cyan and contains significantly less signal in the energy range above $467.5 \mathrm{eV}$. The Ti $L_{2}$ edge is slightly reduced, while there is nearly no influence on the $\mathrm{Ti} L_{3}$ edge.

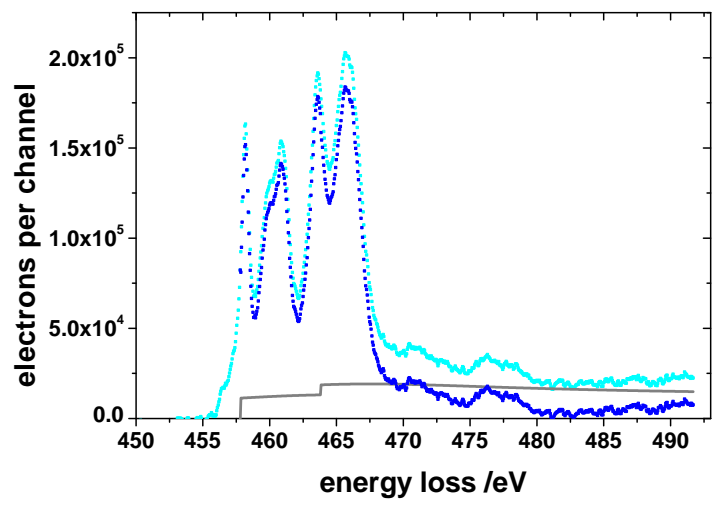

(b) Energy loss spectrum after the deconvolution from fig. 3.16a (cyan). A Hartree-Slater cross-section is aligned (grey) to the cyan spectrum. The subtraction of the Hartree-Slater cross-section results in the final spectrum (blue) in which the $L_{3}-L_{2}$ overlap is corrected.

Figure 3.16.

cross-section was fitted to the region above $467.5 \mathrm{eV}$ to compensate for the $\mathrm{Ti}$ $L_{3,2}$ overlap. Without the deconvolution, the Hartree-Slater adjustment would

\footnotetext{
${ }^{11}$ At least after the binning step the $\mathrm{S} / \mathrm{N}$ ratio should be good enough to use the reflected tail algorithm.
} 
not be accurate. After the deconvolution, the spectrum represents the single scattering distribution of the $\mathrm{Ti} L_{3,2}$ edge.

The binned, pre-edge subtracted and deconvoluted spectra were exported as ${ }^{*}$.msa files from DigitalMicrograph and further evaluated with a self written MATLAB script. The following evaluation steps were performed by this script:

- $L_{3}-L_{2}$ overlap correction: A Hartree-Slater cross-section was fitted to the post edge of the Ti $L_{2,3}$ ELNES; this compensates for the $L_{3}-L_{2}$ overlap. The HartreeSlater cross-section was exported from DigitalMicrograph for the Ti $L$ edge with the appropriate dispersion. The Hartree-Slater cross-section onset was shifted to the Ti $L_{3}$ edge onset ${ }^{12}$ and subsequently scaled in intensity to the continuous cross-section of the Ti $L_{3,2}$ edge above $470 \mathrm{eV}^{13}$. The scaled Hartree-Slater crosssection was subtracted from the Ti $L_{3,2}$ edge. An energy loss spectrum that is corrected for the $L_{3}-L_{2}$ overlap is the result. The described Hartree-Slater cross-section correction of the $\mathrm{Ti} L_{3,2}$ edge overlap is illustrated in fig. 3.16b.

- Envelope function: One Lorentz function was fitted ${ }^{14}$ to each of the four white lines and the one shoulder as shown in fig. 3.17. The five Lorentz functions build up an envelope function that describes the Ti $L$ ELNES appropriately. The envelope function was used to evaluate physical parameters from the $\mathrm{Ti}$ $L$ edges, as discussed in chap. 3.3.3. Since the envelope function is described by 5 analytical (Lorentz) functions, the first derivative was used to find the maxima and the second derivative was used to find the inflection points of the Ti $L$ ELNES. With the fitted envelope function, different parameters of the Ti $L$ ELNES could be determined. One example is the fine structure splitting $\mathrm{d} E\left(b^{\prime}-a\right)$ between the $a$ and $b^{\prime}$ peak as indicated in fig. 3.17.

- Uncertainties and final data evaluation: Up to here the described procedure was applied on every binned data block (blue boxes in fig. 3.14). As a next step, all blocks with the same interface distance $z$ were compared. This allowed for an estimate of the uncertainty that includes all energy drifts present from the monochromator up to the GIF in the time frame of the acquired SI pattern. Literature research has revealed that such an estimate of the uncertainty is new and was not done before or has not been mentioned. Zero distance was chosen to be the point where the signal from the $\mathrm{Ti} L$ edges becomes so poor that no evaluation is possible. This is just before the Ti $L$ edges vanishes in the plural

\footnotetext{
${ }^{12}$ The inflection point of the $\mathrm{Ti} L_{3}$ edge onset, as it is classically defined.

${ }^{13}$ Precisely the 50 lowest values from this energy loss region were taken and averaged. The average was used for the intensity scaling of the Hartree-Slater cross-section. In principle, the number of minimum values that are averaged to calculate the intensity-scaling factor for the Hartree-Slater background can be adjusted in the MATLAB script.

${ }^{14}$ The 'lsqcurvefit' function of MATLAB was used for that purpose. Here, MATLAB uses a nonlinear least-squares solver with the trust-region-reflective algorithms for fitting [102].
} 


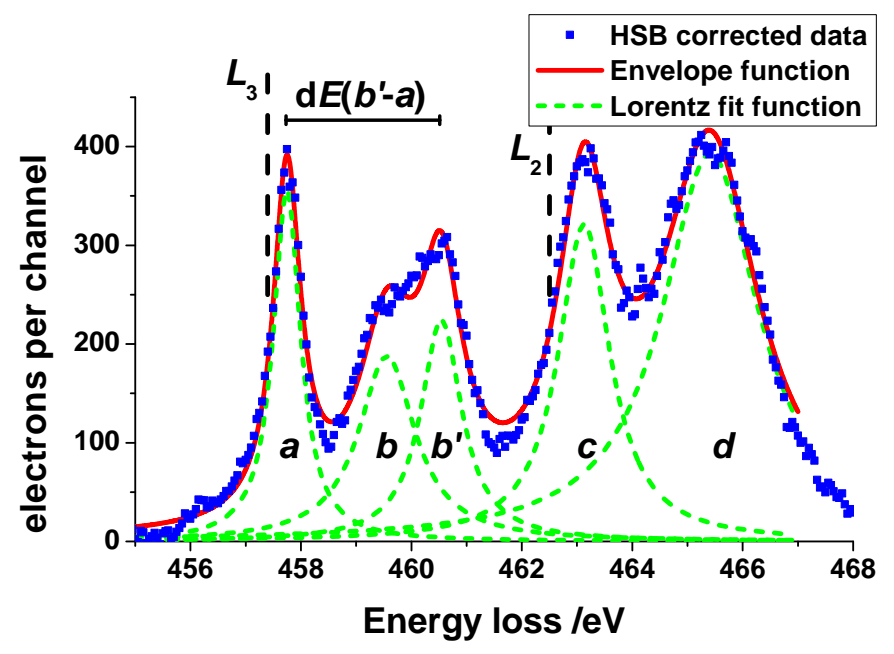

Figure 3.17.: Example of a Hartree-Slater corrected Ti $L_{3,2}$ ELNES (blue). Fitting with 5 Lorentz functions (green dashed lines) gives an envelope function (red) that appropriately describes the features in the Ti $L$ ELNES. The four white lines $a, b / b^{\prime}, c$, $d$ are indicated as well as the fine structure splitting $\mathrm{d} E\left(b^{\prime}-a\right)$ between the maximum of the $a$ and $b^{\prime}$ white line.

scattering background. The beam has therefore already crossed the interface and is partially in the $\mathrm{Pd}$ region.

\subsubsection{Evaluation of physical parameters}

In principle, the evaluation of any feature, as a function of $z$ and of the hydrogen pressure, related to the Ti $L$ ELNES is possible by the automatized evaluation within the MATLAB script. The focus was on the energy loss difference of the peak position of the $a$ and $b^{\prime}$ white line called $\mathrm{d} E\left(b^{\prime}-a\right)$, as is indicated in fig. 3.17 for clarification.

Alternatively, after the described Hartree-Slater correction, all binned data with the same $z$ distance can be averaged and subsequently compared to each other. This 'more classical' approach allows the comparison of ELNES features at different $z$ positions, but at the sacrifice of the error bar. This was used to calculate the corresponding difference spectra that are shown in the results chapter in fig. 4.12. From this added up spectra, the center of gravity of the $b / b^{\prime}$ white line ( $e_{g}$ states) was calculated.

\subsubsection{The center of gravity (CoG)}

Instead of describing the $a$ and $b / b^{\prime}$ white lines with three Lorentz functions, these white lines can be evaluated with a complementarily approach. This is possible with the evaluation of the center of gravity (CoG) of the $a$ and $b / b^{\prime}$ white lines, respectively. The CoGs were used to quantify observed changes as a function of $z$ and of hydrogen 
pressure.

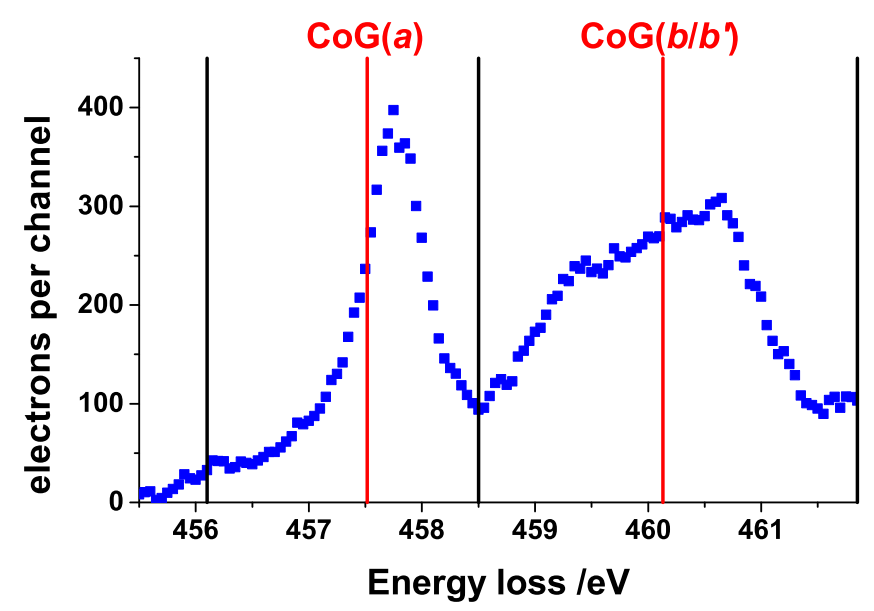

Figure 3.18.: Shown is the $\mathrm{Ti} L_{3}$ edge of the EEL spectrum for $\mathrm{r}-\mathrm{TiO}_{2}$ after all corrections from fig. 3.17. The CoGs (red lines) of the $a$ white line and of the $b / b^{\prime}$ white line were calculated according to eq. 3.1 within the integration windows (black lines). This gives $\operatorname{CoG}(a)=457.615 \mathrm{eV}$ and $\mathrm{CoG}\left(b / b^{\prime}\right)=460.131 \mathrm{eV}$ and according to eq. $3.2 \Delta \mathrm{CoG}=2.516 \mathrm{eV}$.

The $a$ and $b / b^{\prime}$ white lines correspond to $t_{2 g^{-}}$and $e_{g^{-}}$like states, respectively, in a MO picture, see chap. 2.1.2. Thus, calculating the CoG of the $b / b^{\prime}$ white line can be considered as calculating the CoG of the $e_{g}$-like states, or the $\operatorname{CoG}\left(e_{g}\right)$, of the underlying DoS.

The experimental CoG is calculated as

$$
\mathrm{CoG}=\frac{\int I(\Delta E) \cdot \Delta E \mathrm{~d} \Delta E}{\int I(\Delta E) \mathrm{d} \Delta E}
$$

Here, $I(\Delta E)$ are the electrons per channel at an energy loss $\Delta E$, see fig. 3.18. The CoG has units of $\mathrm{eV}$. The integral is calculated over the $a$ and $b / b^{\prime}$ white lines individually, leading to the $\operatorname{CoG}(a)$ and $\operatorname{CoG}\left(b / b^{\prime}\right)$ and $\operatorname{CoG}\left(t_{2 g}\right)$ and $\operatorname{CoG}\left(e_{g}\right)$, respectively. To carry out the CoG evaluation, the $\mathrm{Ti} L_{3}$ spectrum was, therefore, split into the $a$ white line and the $b / b^{\prime}$ white line, as indicated with the black line in the middle of fig. 3.18. Subsequently, eq. 3.1 was applied.

The $\operatorname{CoG}\left(b / b^{\prime}\right)$ can be given with respect to the $\operatorname{CoG}(a)$ by calculating their difference $\Delta$ CoG:

$$
\Delta \mathrm{CoG}=\mathrm{CoG}\left(b / b^{\prime}\right)-\operatorname{CoG}(a)
$$

An example for the whole CoG evaluation is given in fig. 3.18. Here, the CoGs were calculated according to eq. 3.1 with $\operatorname{CoG}(a)=457.615 \mathrm{eV}$ and $\mathrm{CoG}\left(b / b^{\prime}\right)=460.131 \mathrm{eV}$, and are indicated with the red lines. This gives $\Delta \mathrm{CoG}=2.516 \mathrm{eV}$ using eq. 3.2. 
A corresponding uncertainty can be estimated by shifting the boundary between the $a$ and $b / b^{\prime}$ white lines, as shown with the black line in the middle of fig. 3.18. This boundary was shifted to lower energy loss values in the region up to a point that was clearly of $a$ nature and it was shifted to higher energy losses up to a point that was clearly of $b$ nature. This shift of the boundary, gives a measure of the experimental uncertainty of this parameter. The uncertainty of the CoGs can be smaller than the fitting with Lorentz functions, since the noise of the measurement ${ }^{15}$ averages out with the integration procedure. The uncertainty was estimated by shifting the middle boundary by four times of the EELS energy dispersion. Hence, for a dispersion of $0.05 \mathrm{eV} /$ ch this is $0.2 \mathrm{eV}$.

The relative $\operatorname{CoG}\left(b / b^{\prime}\right)$ shift can be calculated by the difference between the $\Delta$ CoG for a certain spectrum and the $\Delta \mathrm{CoG}$ for a reference spectrum:

$$
\text { relative CoG shift }=\Delta \mathrm{CoG}-\Delta \mathrm{CoG}_{\text {reference }}
$$

\subsection{Positron annihilation spectroscopy (PAS)}

Positron annihilation spectroscopy (PAS) measures the gamma radiation that occurs when a positron annihilates with an electron of a solid. The resulting PA spectrum can be quantified to calculate the concentration of open volume, which is related to defects, in the respective material. Details on PAS can for example be found in reviews by Siegel [103] and Č́ižek [104].

PAS is limited to neutrally and negatively charged defects since positrons are repelled by a positive charge. The defects additionally need a sufficient open volume where positron trapping is possible, thus, interstitial defects - as e.g. $\mathrm{Ti}_{\mathrm{i}}$ - can typically not be detected. As an example, the simulated positron density is shown in the $\mathrm{r}-\mathrm{TiO}_{2}(002)$ plane for an oxygen vacancy $\left(\mathrm{V}_{\mathrm{O}}\right)$ and for a Ti vacancy $\left(\mathrm{V}_{\mathrm{Ti}}\right)$ in fig. 3.19. The positron density in a $\mathrm{V}_{\mathrm{Ti}}$ is high with values up to approximately $5 \cdot 10^{-3}$ as shown in fig. 3.19b. In comparison, the positron density of $\mathrm{V}_{\mathrm{O}}$ is significantly smaller, up to approximately $2 \cdot 10^{-4}$, as shown in fig. 3.19a. For a better visualization, the positron density was projected along their maxima in [110] direction. The result is shown in fig. 3.19c. The clear difference between the positron densities of $\mathrm{V}_{\mathrm{Ti}}$ and $\mathrm{V}_{\mathrm{O}}$ become apparent. The positron density of $\mathrm{V}_{\mathrm{Ti}}$ is $15 \times$ higher when compared to the surrounding $\mathrm{r}-\mathrm{TiO}_{2}$ lattice. This is just a factor of two for $\mathrm{V}_{\mathrm{O}}$. The main reason for the low positron density in $\mathrm{V}_{\mathrm{O}}$ is the smaller open volume in comparison to $\mathrm{V}_{\mathrm{Ti}}$. Thus, $\mathrm{V}_{\mathrm{O}}$ cannot and $\mathrm{V}_{\mathrm{Ti}}$ can be measured with PAS with regard to their open volume. It is expected that adding hydrogen in the center of the vacancy will reduce the open volume of $\mathrm{V}_{\mathrm{O}}$ and thus positron trapping is not possible on hydrogen in $\mathrm{V}_{\mathrm{O}}\left(\mathrm{H}_{\mathrm{O}}\right)$ as well.

\footnotetext{
${ }^{15}$ The oscillating amplitude that lies on the intensity signal.
} 


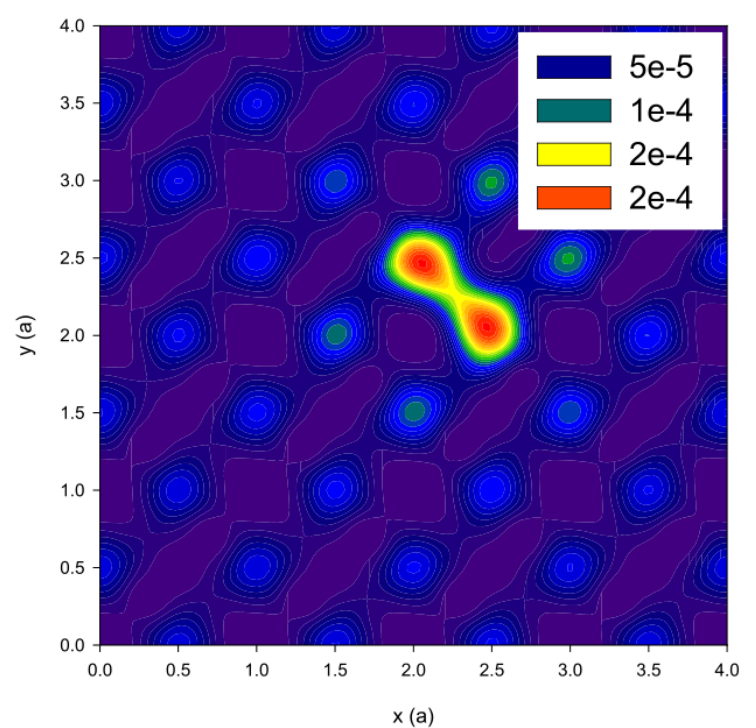

(a)

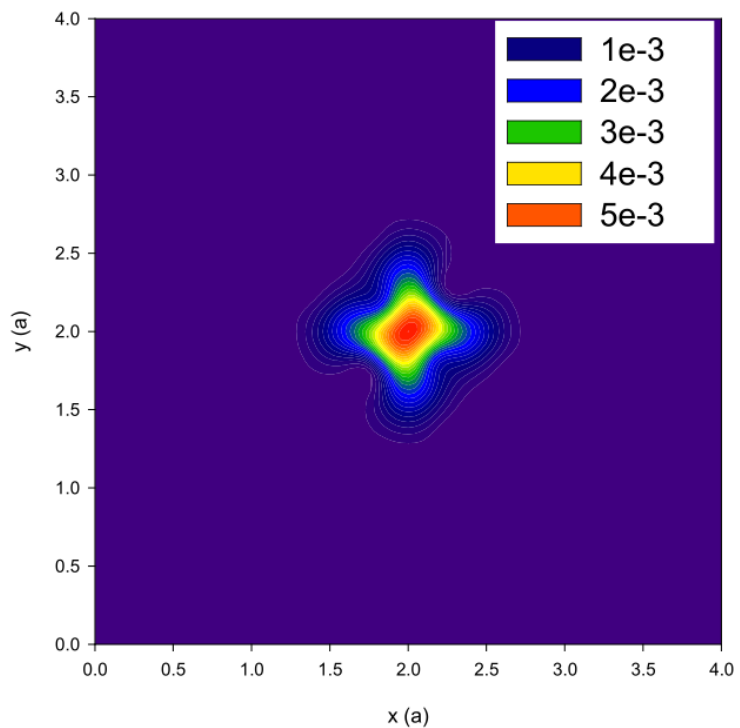

(b)

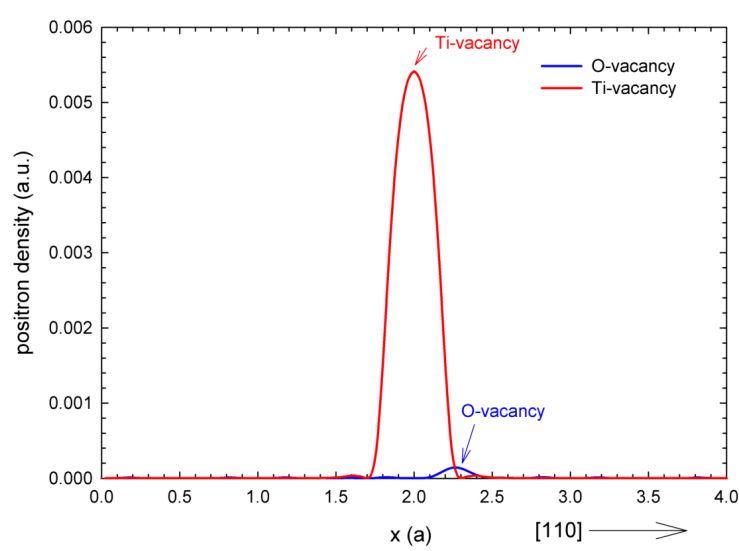

(c)

Figure 3.19.: Positron density in the $\mathrm{r}-\mathrm{TiO}_{2}$ (002) plane for (a) an oxygen vacancy $\left(\mathrm{V}_{\mathrm{O}}\right)$, (b) a Ti vacancy $\left(\mathrm{V}_{\mathrm{Ti}}\right)$ and $(\mathrm{c})$ in a projection along the [110] direction for both vacancies. The vacancies are located at the position of highest positron density as indicated with the red color in (a) and (b). The positron density of $\mathrm{V}_{\mathrm{Ti}}$ is high and of $\mathrm{V}_{\mathrm{O}}$ low. With kind approval of Jakub Č́ízek.

$\mathrm{V}_{\mathrm{Ti}}$ is typically considered as negatively charged. Together with the 'large' open volume positron trapping is possible on $\mathrm{V}_{\mathrm{Ti}}$. As a consequence, PAS can be used to mainly observe a change of the $\mathrm{V}_{\mathrm{Ti}}$ concentrations in $\mathrm{r}-\mathrm{TiO}_{2}$.

PAS investigations of metals differ. But, any open volume defect can be detected by the positrons, including vacancies, dislocations or grain boundaries [104].

In collaboration with Jakub Č́žzek from the Charles University in Prague, variable 
energy positron annihilation spectroscopy (VEPAS) [105] was applied on $\sim 200 \mathrm{~nm}$ $\mathrm{Pd} / \mathrm{r}-\mathrm{TiO}_{2}$ samples (cf. chap. 4.1.2.1) at different hydrogen gas pressures. The pressure range was chosen from $1 \mathrm{~Pa}$ to $1000 \mathrm{~Pa}$ to be comparable to the ETEM experiments.

VEPAS allows, by changing the primary energy of the positrons, a depth analysis: by increasing the primary positron energy, the positron recombination depth is shifted deeper into the material. Thus, in typical VEPAS measurements the evaluated quantity is typically plotted versus the primary energy or the penetration depth. For this work, VEPAS was applied on the $\sim 200 \mathrm{~nm} \mathrm{Pd} / \mathrm{r}-\mathrm{TiO}_{2}$ samples and the interface vicinity while hydrogen was present. This allowed the observation of changes in the $\mathrm{V}_{\mathrm{Ti}}$ concentration as a function of the sample depth and as a function of the hydrogen pressure.

The VEPAS studies were performed on a magnetically guided slow positron beam [106]. The primary energy of the positrons was varied from $80 \mathrm{eV}$ to $35 \mathrm{keV}$. The Doppler broadening of the annihilation photopeak was measured via a HPGe detector with an energy resolution of $1.09 \mathrm{keV}$ at $511 \mathrm{keV}$. The sharpness of the annihilation photopeak was evaluated via the $S$ parameter to characterize the shape of the photopeak [107] which is sensitive to the accessible defect density.

\subsection{X-ray investigation methods}

X-ray diffraction (XRD) and reflectometry (XRR) were used to characterize the crystal orientation, the film texture, the normal strain, the film thickness and the surface/interface roughness of the Pd films that were deposited by magnetron sputtering. In the following a short introduction in XRD and XRR is given as well as some technical details to the evaluation of the performed measurements. A detailed introduction to XRD and XRR is given in the book of Spieß [108].

\subsubsection{X-ray diffraction (XRD)}

X-ray diffraction analysis uses Braggs law [109]:

$$
\frac{(n \cdot \lambda) / 2}{d_{h k l}}=\sin \left(\frac{2 \Theta_{h k l}}{2}\right) \Leftrightarrow n \cdot \lambda=2 d_{h k l} \cdot \sin \left(\frac{2 \Theta_{h k l}}{2}\right)
$$

where $n$ is the diffraction order, $\lambda$ the wavelength of the incident beam, $d_{h k l}$ the lattice plane distance between the $(h k l)$ planes in normal direction and $\Theta_{h k l}$ the Bragg angle (scattering angle). Bragg's law is shown schematically in fig. 3.20. An incident beam is diffracted at the $(h k l)$ lattice planes of an crystal with distance $d_{h k l}$. The diffracted beam interferes constructively if eq. 3.4 is fulfilled and the corresponding diffracted beam travels a path difference of $(m-1) \cdot 2 d_{h k l} \cdot \sin \left(\frac{2 \Theta_{h k l}}{2}\right)$, where $m$ is the m'th $(h k l)$ plane counted from the top. In a diffractometer, the intensity of the scattered x-rays is measured as a function of $2 \Theta$. If constructive interference at lattice planes appears, 


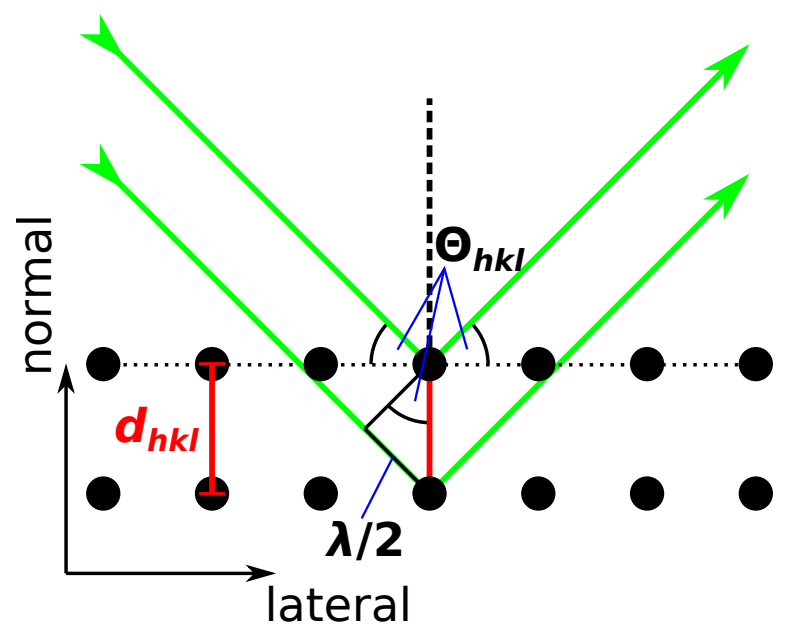

Figure 3.20.: Sketch of Bragg's law, cf. eq. 3.4. The incident x-rays interfere constructively if the drawn relationship is fulfilled. This yields to an interference maximum that can be measured with a detector. Indicated are the wavelength of the incident beam $\lambda$, the lattice plane distance $d_{h k l}$, the half path difference $(\lambda / 2)$ necessary for the constructive interference and the normal and the lateral directions.

the corresponding diffractogram shows an interference maximum which corresponds to the $(h k l)$ plane that meets Bragg's law, cf. eq. 3.4.

XRD was used for determining the normal orientation and the texture (cf. chap. 3.5.1.4) of the $\mathrm{Pd}$ films deposited on the $\mathrm{r}-\mathrm{TiO}_{2}$ crystals. In the following the evaluation procedure of the diffractograms is described.

The taken XR diffractograms deliver the $2 \Theta$ value for the maxima of the measured interferences. To gain the maxima, the spectra were evaluated in the following way:

1. The background was subtracted in Bruker's EVA (v3.1) software and subsequently exported as a '.xzy' file.

2. The data were imported to OriginPro (v8.5.0) from the OriginLab Corporation (Northampton, USA) and evaluated using the 'Peak Analyzer' tool.

3. The diffractogram was aligned by the $\mathrm{r}-\mathrm{TiO}_{2}(110)$ and/or $\mathrm{r}-\mathrm{TiO}_{2}(220)$ interference with respect to their literature values ${ }^{16}$.

4. Voigt functions were used for fitting the interferences:

$$
y=y_{0}+A \frac{\left(2 \ln (2) w_{L}\right)}{\left(\pi^{1.5} w_{G}^{2}\right)} \int_{-\infty}^{+\infty} \frac{e^{-t^{2}}}{\left(\sqrt{\ln (2)} \frac{w_{L}}{w_{G}}\right)^{2}+\left(\sqrt{4 \ln (2) \frac{\left(x-x_{c}\right)}{w_{G}}}-t\right)^{2}} d t
$$

5. The $2 \Theta_{h k l}$ were extracted from the fits. If necessary, they were used for further evaluations. The given uncertainties were taken from the uncertainties of the fit.

${ }^{16}$ Of course, this yields to a deviation of the corundum alignment of the D8 Discoverer. It corresponds, more or less, to an alignment of the spectrum to a $\mathrm{r}-\mathrm{TiO}_{2}$ reference. Though it was found that this is a precise way to align the $\mathrm{Pd}(111)$ interference which is in between the $\mathrm{r}-\mathrm{TiO}_{2}(110)$ and $\mathrm{r}-\mathrm{TiO}_{2}(220)$ interference. 


\subsubsection{XR diffractogram of a $\mathrm{Pd}(111)$ single crystal}

This section presents an example for a XRD measurement and the corresponding evaluation.

XRD was applied on a $\operatorname{Pd}(111)$ single crystal from the MaTecK GmbH (Germany, Juelich) with dimensions of $\varnothing 10 \mathrm{~mm} \times 2 \mathrm{~mm}$. The corresponding XR diffractogram plots the measured XR intensity in counts per second (cps) against the diffraction angle $2 \Theta$ in $^{\circ}$ and is shown in fig. 3.21. Two main interferences are visible and correspond to

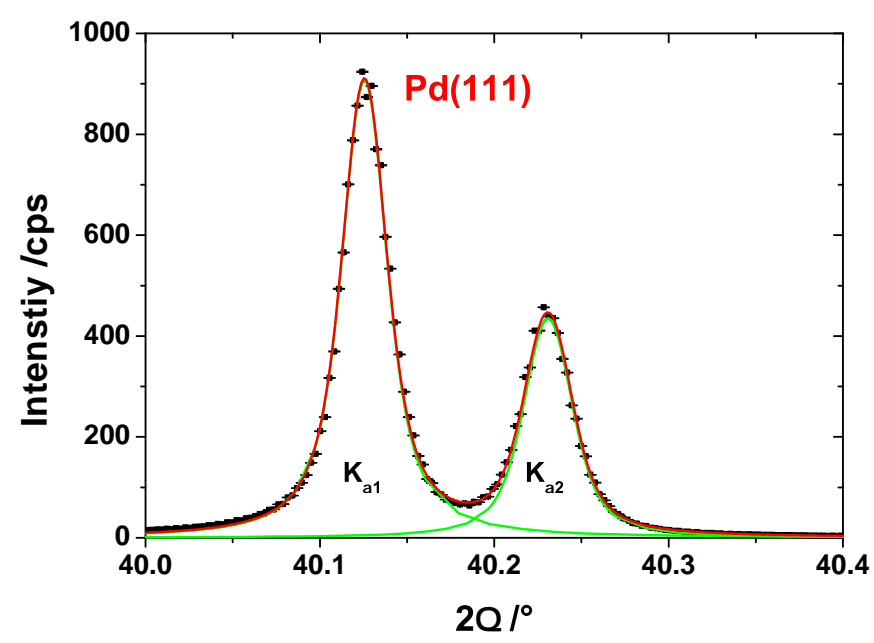

Figure 3.21.: XR diffractogram of an $\mathrm{Pd}(111)$ single crystal. The measured intensity is shown versus $2 \Theta$. The selected angle range shows the $\operatorname{Pd}(111)$ interference for the $\mathrm{Cu} K_{\alpha_{1}}$ and $K_{\alpha_{2}}$ line (black points). Each interference was individually fitted with a Voigt function (green lines) via OriginPro. The total envelope function is shown with the red line.

the $\mathrm{Cu} K_{\alpha_{1}}$ and $K_{\alpha_{2}}$ diffraction on the $\mathrm{Pd}(111)$ lattice planes. Fitting the diffractogram with two Voigt functions allows the determination of the interference position $2 \Theta_{111}$ for each wavelength: $2 \Theta_{111}\left(\mathrm{Cu} K_{\alpha_{1}}\right)=(40.1256 \pm 0.0003)^{\circ}$ and $2 \Theta_{111}\left(\mathrm{Cu} K_{\alpha_{2}}\right)=$ $(40.2309 \pm 0.0006)^{\circ}$. Applying Braggs law, cf. eq. 3.4, by using the wavelengths of $\mathrm{Cu}$ $K_{\alpha_{1}}=1.540598 \AA$ and $\mathrm{Cu} K_{\alpha_{2}}=1.544426 \AA$, a weighted average (cf. appendix A.7) for $d_{111}^{0}$ can be calculated:

$$
d_{111}^{0}=(2.24543 \pm 0.00003) \AA
$$

The value is slightly smaller than the value given by Arblaster [72] with $d_{111}^{0}=$ $(2.2460 \pm 0.0004) \AA$ for bulk Pd. The reason can be a small deviation of the XRD system from the corundum reference ${ }^{17}$ or a reduced defect density in the single crystal in comparison to the bulk system.

\footnotetext{
${ }^{17}$ The uncertainty given above does not include the absolute deviation from the corundum reference since the measurements are all done with the same setup on the same machine.
} 
A self-measured reference at the XR system is always of advantage since the $\mathrm{Pd}$ interferences measured in XRD for the thin films can be directly compared to it. Though, unfortunately, the Pd single crystal was not always available during this thesis and additionally the D8 Discoverer was realigned a couple of times. Thus, for simplicity, always the value of Arblaster [72] was used as the reference.

\subsubsection{Normal strain in thin films}

The strain in normal direction $\epsilon_{\text {normal }}$ of the Pd films can be calculated from XRD interference shifts.

The Pd films deposited on the $\mathrm{r}-\mathrm{TiO}_{2}(110)$ crystals exhibit only an (111) orientation in normal direction (cf. chap. 4.1.2). Using XRD, $d_{111}$ can be measured via the $\operatorname{Pd}(111)$ interference maximum and compared to $d_{111}^{0}$ given from Arblaster [72]. Rearranging eq. 3.4 to the measured $\operatorname{Pd}(111)$ lattice plane distance - assuming the main interference $(n=1)$ - yields

$$
d_{111}=\frac{\lambda}{2 \cdot \sin \left(\frac{2 \Theta_{111}}{2}\right)} .
$$

The strain in the normal direction of the $\mathrm{Pd}$ film is then calculated via

$$
\epsilon_{\text {normal }}=\frac{\Delta d_{111}}{d_{111}^{0}}=\frac{d_{111}-d_{111}^{0}}{d_{111}^{0}}
$$

In specific cases, the lateral stress state of thin films on substrates can be directly calculated from $\epsilon_{\text {normal }}$, by assuming a specific stress state such as biaxial [110]. The shift of the $\operatorname{Pd}(111)$ interference is sufficient for this determination of the film's stress state [108, chap. 10.4] if

- all grains contribute to the measured interference,

- diffraction occurs in the whole film depth (penetration depth) and

- the elastic properties are isotropic in lateral direction.

In principle, these constraints are satisfied for a $\operatorname{Pd}(111)$ film as it is discussed in appendix A.4. However, the $\mathrm{r}-\mathrm{TiO}_{2}$ surface is anisotropic and therefore a biaxial lateral stress state cannot be assumed. Consequently, an estimate of the lateral stress state of the $\mathrm{Pd}$ films via the $\operatorname{Pd}(111)$ interference shift and $\epsilon_{\text {normal }}$, respectively, is not possible due to the anisotropy of the $\mathrm{r}-\mathrm{TiO}_{2}(110)$ surface.

\subsubsection{Stress free lattice parameter of thin Pd film}

The stress free lattice parameter(s) for thin films typically differs from the lattice parameter of the bulk material. The deviation from the bulk values occurs due to a higher density of defects in the thin films that depend on the deposition conditions. 
Thus, $d_{111}^{0}$ can be different for a thin (Pd) film compared to the value of the bulk material from Arblaster [72] which can influence the calculation of the normal strain $\epsilon_{\text {normal }}$, cf. eq. 3.6. Literature investigations of the stress free lattice parameter of thin Pd films deposited at temperatures around $1023 \mathrm{~K}$ is not available. However literature is available for thin $\mathrm{Pd}$ films deposited at RT.

Harumoto et al. [111] have performed an approach to measure the strain free lattice parameter for thin $\mathrm{Pd}$ film:

With sputtering deposition they prepared Pd films with $8 \mathrm{~nm}, 16 \mathrm{~nm}, 24 \mathrm{~nm}$ and $48 \mathrm{~nm}$ thickness at RT. XR diffractograms were taken at a $\psi$ angle of $0^{\circ}$ and $70.5^{\circ}$. The spectra were evaluated combining a rhombohedral distortion analysis with a diffraction stress analysis. Harumoto et al. calculated the stress free lattice parameter of their thin Pd films. For all films, the deviations from the bulk lattice parameter were found to be within the uncertainty of the measurement and thus $\lesssim 0.1 \%$.

Applying a deviation of $0.1 \%$ to the $\mathrm{Pd}$ bulk lattice parameter $a$ given by Arblaster [72] yields to $d_{111}$ being in the approximate interval

$$
[2.2438 \AA ; 2.2483 \AA] \text {. }
$$

This is an estimate of the stress free lattice parameter of thin Pd films sputtering deposited at RT.

However, for the prepared Pd film, the defect density is expected to be lower since the produced Pd films were deposited at high temperatures where defects anneal more easily. Thus, the stress free lattice parameter of the $\mathrm{Pd}$ film produced is expected to deviate less from the bulk value. The measured $d_{111}$ values for the $\sim 100 \mathrm{~nm} \mathrm{Pd}$ films were found to be out of the given interval and thus a quantitative investigation of the stress free lattice parameter was not necessary for these films.

\subsubsection{Pole figures and $\phi$ scans}

Pole figure measurements are an extended form of XRD. In addition to conventional XRD measurements, in a pole figure measurement, the sample is tilted in $\chi$ and $\omega$ to cover a half (or a smaller part of a) sphere around the sample's surface. The results is a stereographic projection of the measured interferences. Details about pole figure measurements can be found in the book of Spieß [108, chap. 11].

For a $\phi$ scan, $\psi, 2 \Theta$ and $\Theta$ are fixed and just the $\phi$ circle is rotating. Thus, a $\phi$ scan corresponds to a 'ring' in the pole figure whose radius is defined by the $\psi$ angle. The advantage of $\phi$ scans are that they can be carried out in high resolution while the resolution is low in the case of pole figure measurements.

Pole figures were used to get information about the growth of $\mathrm{Pd}$ on the $\mathrm{r}-\mathrm{TiO}_{2}$ crystal: preferred orientations can be revealed and the orientation relationship to the 
substrate can be determined. To obtain a full orientation distribution function (ODF) three Pd pole figures were measured: (111), (200) and (220).

$\phi$ scans were used in addition to pole figures to determine the orientation relationship of the $\mathrm{Pd}$ films on the $\mathrm{r}-\mathrm{TiO}_{2}(110)$ crystals.

The pole figure measurements were evaluated with the MulTex (v3) software from Bruker. MulTex allows for fitting a whole orientation distribution function to a set of measured pole figures of one $\mathrm{Pd}$ film.

The pole figures were measured and simulations in MulTex were carried out in collaboration with Philipp Klose.

For the pole figure measurements the following setup was used: point focus of the X-ray source, $9 \mathrm{~mm}$ motorized primary slit, $2 \mathrm{~mm}$ pin hole, $9 \mathrm{~mm}$ motorized secondary slit and fully open Lynxeye detector in 1D mode.

\subsubsection{X-ray reflectometry (XRR)}

In contrast to XRD where the x-rays interfere on the lattice planes, in x-ray reflectometry (XRR) the interference appears between different layers of a layered system. Here, the film thickness, the interface and the surface roughnesses and the density of the layers influence the interference. Hence, all these quantities can be measured with XRR. These parameters can be measured over a sample range of $\mathrm{mm}^{2}$ whereas techniques such as AFM, cf. chap. 3.7, are limited to the $\mu \mathrm{m}^{2}$ range. XRR has the additional benefit of a high sensitivity for thin layers with thicknesses even below nm. Such thin layers have long range oscillations in $2 \Theta$ space and can be precisely measured. This can reveal thin oxide layers on metal surfaces or intermediate phases between film and substrate. XRR has also some restrictions: XRR can be used up to $2 \Theta=10^{\circ}$. However, typical measurements end below $2 \Theta=8^{\circ}$. Additionally, the $2 \Theta$ resolution and the absorption of the investigated materials limits the maximum film thickness that can be addressed.

$\mathrm{XRR}$ is an appropriate tool to investigate the $\sim 10 \mathrm{~nm} \mathrm{Pd} \mathrm{films} \mathrm{produced.} \mathrm{The}$ recorded XRR spectra were evaluated with the software LEPTOS (v7.7) from Bruker. The simulation of the measured XRR spectra fits the film thickness, the interface and surface roughnesses and, if desired, the density ${ }^{18}$. The roughness is described with a Gaussian model that describes the roughness as a deviation from the average film thickness. Therefore the roughness is given as a root mean square roughness (RMS) and can be directly compared to the surface roughness evaluated from AFM measurements (see chap. 3.7).

The maximum film thickness $h_{\max }$ that can be investigated by XRR is connected to the resolution $\Delta \Theta \approx 0.01^{\circ}$ of the used system ${ }^{19}$. For two neighbouring maxima $(m$

\footnotetext{
${ }^{18}$ Though to determine the density highly resolved XRR measurements with up to $8^{\circ}$ or even up to $10^{\circ}(2 \Theta)$ are necessary. The densities were not evaluated.

${ }^{19}$ The resolution in XRR is in principle not a fixed value but depends on the sample and on the used setup of the XR system. It can be accurately measured by a $\omega$ and $\Theta$ scan, respectively, in total
} 
and $n$ ) and with $\mathrm{Cu} K_{\alpha_{1}}$ radiation this yields to [108, eq. 13.8]

$$
h_{\max }=\frac{\lambda(m-n)}{2\left(\sin \left(\Theta_{m}\right)-\sin \left(\Theta_{n}\right)\right)} \approx 440 \mathrm{~nm} .
$$

In addition, the material can limit $h_{\max }$ by absorption, especially Pd with its high density. This is an important issue if the $\mathrm{Pd} / \mathrm{r}-\mathrm{TiO}_{2}$ interface is of interest: The interface cannot be characterized via XRR if the Pd absorbs the X-rays and the underlying $\mathrm{r}-\mathrm{TiO}_{2}$ substrate is not visible. The related thickness of a material is called the maximum penetration depth $\Lambda_{\max }$. With the extinction coefficient $\beta$, cf. chap. 2.2, the order of magnitude of $\Lambda_{\max }$ for XRR measurements can be estimated as [78, slide 15]:

$$
\Lambda_{\max } \approx \frac{\lambda}{2 \pi \sqrt{\beta}} \approx 14.2 \mathrm{~nm}
$$

Thus, in an XRR measurement of a Pd film with thicknesses a view tenth of nanometers, any substrate or underlying film(s) will be invisible. Experimentally it was found that the substrate was invisible for $\mathrm{Pd}(111)$ films with a thickness of $37 \mathrm{~nm}$ and visible with a thickness of $28 \mathrm{~nm}$. Therefore $\Lambda_{\max }$ for $\mathrm{Pd}$ is in between these values and thus approximately $30 \mathrm{~nm}$. The corresponding XRR measurement are attached in the appendix in fig. A.2 and in tab. A.1 for the $28 \mathrm{~nm}$ and in fig. A.8 and in tab. A.2 for the $35 \mathrm{~nm}$ film.

\subsubsection{Diffractometer details}

For this work, the high resolution diffractometer 'D8 Discoverer' from the Bruker Corporation (USA, Billerica) was used. The $2 \Theta$ circle of the D8 is aligned to a corundum reference that was delivered with the system. The setup is shown in fig. 3.22. The X-ray source (1) is a type KFL Cu $2 \mathrm{~K}$ DC from Siemens and uses a copper $(\mathrm{Cu})$ anode. It is typically powered with $40 \mathrm{kV}$ and $40 \mathrm{~mA}$ and emits three major wavelength ${ }^{20}$ in a line focus: $\mathrm{Cu} K_{\alpha_{1}}$ with $1.5405931(5) \AA, \mathrm{Cu} K_{\alpha_{2}}$ with $1.5444276(5) \AA$ and $\mathrm{Cu} K_{\beta_{1}}$ with 1.392234(6) $\AA$ [76]. The X-rays first hit the Goebel mirror (2) where the beam is parallelized in the $2 \Theta$ plane. The Goebel mirror additionally cuts out most of the $\mathrm{Cu} K_{\beta}$ line, though for single crystals it is still detectable. The parallelized beam traverses the primary slit (3) which defines the illuminated area (in beam direction) and (partially) the resolution of the instrument. A beam monochromator (4) can be used to cut out the $\mathrm{Cu} K_{\alpha_{2}}$ and the rest of the $\mathrm{Cu} K_{\beta}$ line. The use of the monochromator

reflection. The resolution $\Delta \Theta$ is defined as FWHM/2 of a fitted Gaussian distribution to this measurement.

${ }^{20}$ Doing XRD on single crystals can reveal additional wavelengths. Typically tungsten lines become present as the source ages: A part of the tungsten cathode is evaporated and deposited on the $\mathrm{Cu}$ anode resulting in the presence of tungsten $L$ lines between the $\mathrm{Cu} K_{\alpha}$ and $\mathrm{Cu} K_{\beta}$ lines. With the use of a monochromator the tungsten lines can be suppressed by sacrificing primary intensity. 


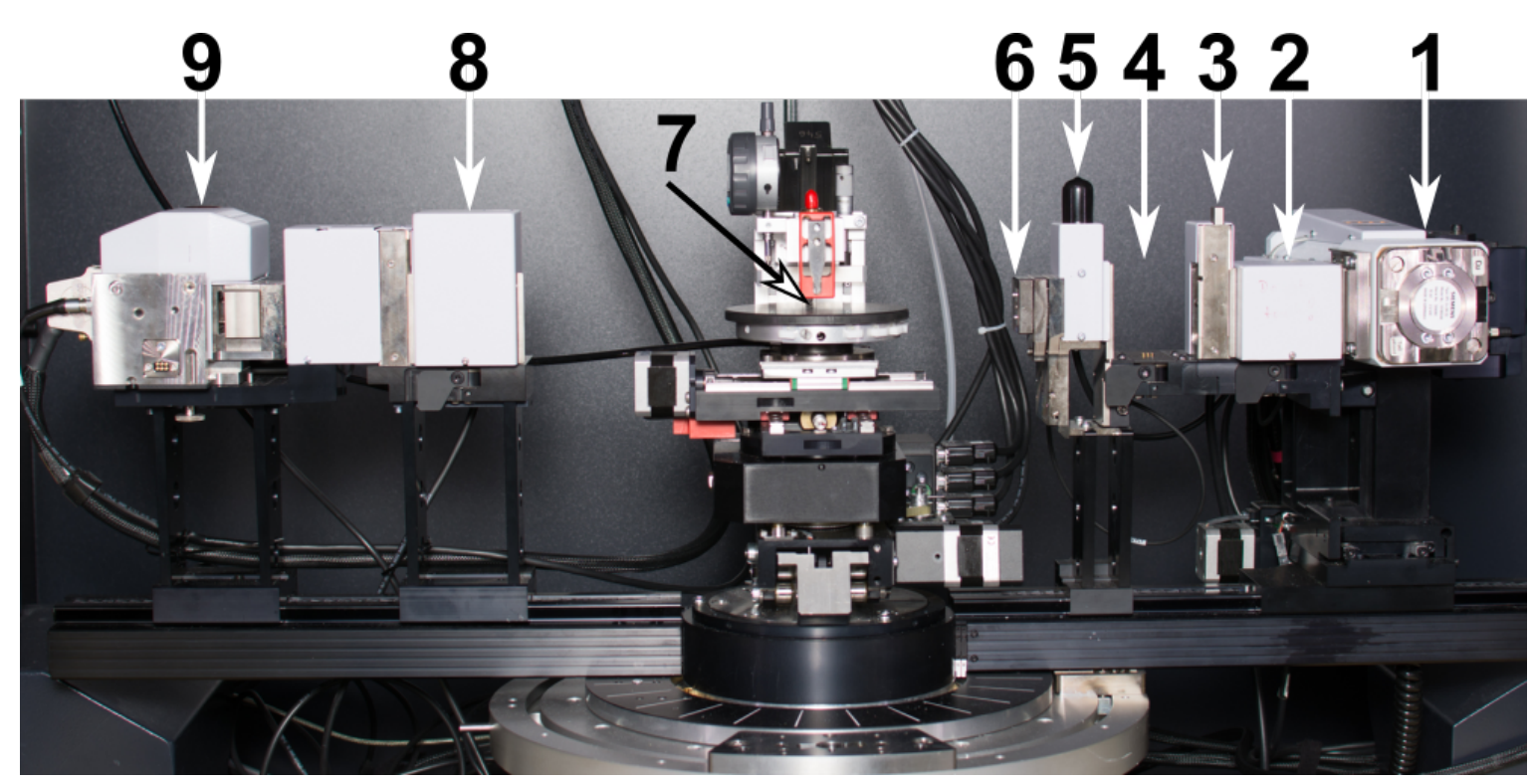

Figure 3.22.: The used Bruker D8 Discoverer. The parts are denoted in the text.

typically reduces the primary intensity significantly. An absorber (5) can reduce the $\mathrm{X}$-ray photon flux to prevent the detector from coming into its dead time. With an additional mask (6) the height divergence of the beam can be cut, thus the sample is not over illuminated on its top and bottom part. The beam next passes the goniometer center with the plate (7) where the sample is mounted. The sample is typically held in place by a negative pressure suction. The diffracted or reflected beam next passes a motorized slit (8) in the secondary beam optics. Before the beam runs into the detector it passes the detector slit (9). The Lynxeye detector (9) is capable of measuring in 0D mode, where the motorized and detector slit define the illuminated detector area and therefore also the resolution, or in 1D mode, in which every of the 192 detector channels (oriented in $2 \Theta$ direction as a comb) is read out individually and both motorized and detector slit are fully open. The 1D mode is typically used for XRD measurements, since it has a superior speed, and the 0D mode is used for the alignment procedure and for XRR measurements. All sample surfaces are aligned parallel to the center of the primary beam before the measurements.

For the XRD measurements, the following setup is used unless otherwise stated: Goebel mirror, $0.1 \mathrm{~mm}$ primary slit, no monochromator, $2 \mathrm{~mm}$ or $4 \mathrm{~mm}$ mask, $9 \mathrm{~mm}$ motorized secondary slit and fully open Lynxeye detector in 1D mode.

For XRR measurements the setup is slightly different: Goebel mirror, $0.1 \mathrm{~mm}$ primary slit, no monochromator, $2 \mathrm{~mm}$ or $4 \mathrm{~mm}$ mask, $0.075 \mathrm{~mm}$ motorized secondary slit and $0.075 \mathrm{~mm}$ detector $\mathrm{slit}^{21}$ with the Lynxeye detector in $0 \mathrm{D}$ mode.

${ }^{21}$ This corresponds to a illumination of just the central detector channel. 


\subsection{X-ray photoelectron spectroscopy (XPS)}

In x-ray photoelectron spectroscopy (XPS) x-rays are focused on a sample surface. There, the x-rays excite electrons from different atomic shells. The number and kinetic energy of these electrons are measured and allow the analysis of the binding energy of these electrons in the material. Hence, XPS allows for elemental as well as for surface chemistry analysis. The information depth of XPS is limited to approximately the first $10 \mathrm{~nm}$ of the sample's surface. An XP spectrum shows the number of collected electrons as a function of their binding energy in $\mathrm{eV}$. A detailed introduction to XPS can be found in the book of Watts and Wolstenholme [112].

XPS was applied to investigate the surface of the $\mathrm{r}-\mathrm{TiO}_{2}$ crystals in the as-delivered state and after the pretreatment (cf. chap. 3.1.1). The measurements were performed in collaboration with Julius Scholz and Niklas Herwig [93].

\subsection{Atomic force microscopy (AFM)}

Atomic force microscopy (AFM) [113] uses a nanoprobe which is connected to a cantilever to probe the surface of a material. The probe is a tip that has dimensions in the $\mathrm{nm}$ range. The tip scans the surface by being in a mechanical interaction with the sample's surface. The cantilever bends due to the morphology of the surface. The bending is measured with a detector and delivers height information about the sample's surface. This results in height profiles of the surface. AFM additionally allows for measuring forces on the surface or manipulation of the surface. More details about AFM can be found in the book of Haugstad [114].

For this work, AFM was used to measure the surface topography of the samples. This was either used for the $\mathrm{r}-\mathrm{TiO}_{2}$ crystals or the thin $\mathrm{Pd}$ film. The gained topography data allowed for a further evaluation to calculate the surface roughness in the area measured with AFM. Here, typical lateral scan windows had $10 \times 10 \mu^{2}$ size or below. The measurements were performed in tapping mode, which is also known as intermittent or AC mode.

The AFM data were displayed and evaluated with the software Gwyddion [115]. Gwyddion can calculate the root mean square roughness [116]. This value is comparable to the roughness measured with XRR (cf. 3.5.2). The roughness from AFM can be limited if the tip radius of the cantilever is larger than the structures on the sample surface. Thus, the surfaces may look less rough in AFM and the obtained roughness can be seen as a lower boundary for the surface roughness. In addition, AFM can show extracts of the sample surface in the $\mu^{2}$ range.

The AFM used for this work is a MFP-3D ${ }^{\mathrm{TM}}$ connected to the SPM controller ARC2 ${ }^{\mathrm{TM}}$ from Oxford Instruments Asylum Research, Inc. (Santa Barbara, USA). The lateral resolution of the AFM is approximately $0.5 \mathrm{~nm}$ and the height resolution is approximately $0.2 \mathrm{~nm}$ [117]. AFM probes of the type AC160TS [118] from Oxford 
Instruments were used, which have a tip radius of approximately $7 \mathrm{~nm}$.

Typical used parameters for the AFM measurements are: a scan speed between $10 \mathrm{\mu m} / \mathrm{s}$ and $0.5 \mathrm{\mu m} / \mathrm{s}, 512$ or 1024 points \& lines, a set point of $800 \mathrm{mV}$, an integral gain $^{22}$ that was chosen as high as possible but low enough to prevent ringing noise, a driving amplitude ${ }^{23}$ that was high enough to stay in the repulsive regime $\widehat{=}$ (phase $<$ $90^{\circ}$ ) in the whole scanned area.

\subsection{Density functional theory (DFT) on the hydrogen-r- $\mathrm{TiO}_{2}$ system}

Density functional theory (DFT) is based on the exact Hohenberg Kohn Theorem [119] and is a method to describe quantum mechanical properties of a many-body system. Examples of such systems are molecules and solids. DFT predicts the energy and the electron density of the electronic ground state of these systems. To do so, in DFT, the Schrödinger equation [120] is solved with the use of the density functional which expresses the interacting electrons in terms of non-interacting electrons in an effective potential. The density functional can be derived in a general form without any approximation. However, the exact density functional is not known. Hence, in practice, approximate functionals (PBE, PBE0r, etc.) are used.

The DFT and interface calculations were carried out by M. Sotoudeh and P. Blöchl in close collaboration with the author. A legal statement is given at the end of this section which clarifies the individual contributions to this collaboration.

DFT was applied to investigate changes accompanied by the introduction of defects to (undoped) $\mathrm{r}-\mathrm{TiO}_{2}$. The calculations addressed oxygen and hydrogen related defects in different charge states: the doubly positive, positive and neutral oxygen vacancy $\left(\mathrm{V}_{\mathrm{O}}^{2+,+, 0}\right)$; the positive, neutral and negative interstitial hydrogen $\left(\mathrm{H}_{\mathrm{i}}^{+, 0,-}\right)$; and the positive, neutral and negative substitutional hydrogen $\left(\mathrm{H}_{\mathrm{O}}^{+, 0,-}\right)$. The DFT investigations focused on the thermodynamics, the atomic structure of the defect and on the changes that were introduced to the unoccupied part of the DoS by the defects. Such defect-induced changes to the unoccupied DoS have not been addressed in the literature $[50,57]$. The changes in the unoccupied DoS can in principle be related to EELS and can contribute to an understanding of the observed changes in EEL spectra.

To study the thermodynamics, the formation energies $\Delta E_{\mathrm{f}}$ of the different defect species were investigated. The formation energy $\Delta E_{\mathrm{f}}[X]$ of a respective defect $X$ is defined as:

$$
\Delta E_{\mathrm{f}}[X]=E\left[\mathrm{r}-\mathrm{TiO}_{2}+X\right]-E\left[\mathrm{r}-\mathrm{TiO}_{2}\right]-\sum_{i \in\{\mathrm{e}, \mathrm{H}, \mathrm{O}\}} \mu_{i} \eta_{i, X}
$$

\footnotetext{
${ }^{22}$ Typical values are between 6 and 14 but mainly around 10

${ }^{23}$ Typically between $30 \mathrm{mV}$ and $500 \mathrm{mV}$ depending on the maximal height change in the investigated area.
} 
Here, $E\left[\mathrm{r}-\mathrm{TiO}_{2}\right]$ denotes the energy of the undoped supercell, $E\left[\mathrm{TiO}_{2}+X\right]$ denotes the energy of the supercell containing an additional defect $X, \mu_{i}$ denotes the chemical potential of electrons (e), oxygen atoms $(\mathrm{O})$ and hydrogen atoms $(\mathrm{H})$ and $\eta_{i, X}$ denotes the corresponding number of particles of species $i$ that was added from the reservoir to form the defect $X$. The chemical potentials of oxygen $\mu_{\mathrm{O}}$ and hydrogen $\mu_{\mathrm{H}}$ were given relative to the molecular species. The formation energies were evaluated as a function of the chemical potential of the electrons $\mu_{\mathrm{e}}$. The formation energies can be equal for one defect in two different charge states $q_{1}$ and $q_{2}$. These crossing points define the thermodynamic charge state level $\epsilon\left(q_{1} / q_{2}\right)$ :

$$
\epsilon\left(q_{1} / q_{2}\right)=\frac{E\left[\mathrm{TiO}_{2}+X^{q_{2}}\right]-E\left[\mathrm{TiO}_{2}+X^{q_{1}}\right]}{q_{2}-q_{1}}
$$

The charge state level therefore gives the energy for $\mu_{\mathrm{e}}$ at which the charge states $q_{1}$ and $q_{2}$ coexist.

In a thermodynamic model, the concentration $c_{i}$ per oxygen site of the species $i$ in $\mathrm{r}-\mathrm{TiO}_{2}$ was studied as a function of $\mu_{\mathrm{O}}{ }^{24}$. For that purpose the grand potential $\Omega$ was defined by the contribution of all defects and the (free) electrons. In the thermodynamic model, the defects were described in a multistate model which means that an oxygen site can be occupied by oxygen, as in the undoped material, or by one of the (charged) defects $\left(\mathrm{H}_{\mathrm{i}}^{+, 0,-}, \mathrm{V}_{\mathrm{O}}^{2+,+, 0}\right.$ or $\left.\mathrm{H}_{\mathrm{O}}^{+, 0,-}\right)$. The defect concentrations were calculated considering formation energies and configurational entropies.

Based on the thermodynamic model, a $\mathrm{Pd} / \mathrm{r}-\mathrm{TiO}_{2}$ interface was simulated in an electrostatic picture solving Poisson's equation in a self-consistent cycle. The charge density in Poisson's equation was calculated from the derivative of the grand potential with respect the chemical potentials. For that purpose, a Schottky barrier $\Phi_{\mathrm{B}}^{0}$ of $1 \mathrm{eV}$ was assumed between $\mathrm{Pd}$ and $\mathrm{r}-\mathrm{TiO}_{2}$. For the calculation of the interface, the charge density was fixed on the metal side by $\operatorname{Pd}(z<0)$, and far away from the interface on the $\mathrm{r}-\mathrm{TiO}_{2}$ side by the doped $\mathrm{r}-\mathrm{TiO}_{2}(z \gg 0)$. The charge and defect densities therefore align between both sides. In this framework, the defect concentration (per oxygen site) at the $\mathrm{Pd} / \mathrm{r}-\mathrm{TiO}_{2}$ interface was studied as a function of the oxygen $\mu_{\mathrm{O}}$ and hydrogen $\mu_{\mathrm{H}}$ chemical potential which were part of $\Omega$.

Based on the results of the DFT calculations, the atomic structure was constructed. The atomic defect environment was investigated with respect to the local symmetry and the local orbital arrangement.

The defect induced changes in the unoccupied DoS were studied by plotting different kinds of DoS. In general, the DoS shows the number of states per energy against the energy $E$ of the calculation. In every DoS, the zero point of $E$ was chosen to be at the valence band maximum.

To study the DoS changes, two kinds of DoS were used: the DoS of the whole supercell and a partial DoS. The DoS of the whole supercell was investigated for

\footnotetext{
${ }^{24} \mu_{\mathrm{O}}$ is related to the experimentally applied oxygen partial pressure.
} 
undoped $\mathrm{r}-\mathrm{TiO}_{2}$. It contained the total DoS which projects the DoS on all used orbitals $^{25}$ and selected DoS's that were projected to specific orbitals ${ }^{26}$. The partial DoS was calculated for the $d$ orbitals of the (three) Ti atoms that were adjacent to the defect (center). In the partial DoS, the individual DoS contributions were projected onto specific orbitals. For the projection of the partial DoS, the projection axis was chosen as the O-Ti-defect center axis.

The calculated defect induced changes in the unoccupied DoS were quantified applying the center of gravity $(\mathrm{CoG})$. The CoGs are defined as the mean value of corresponding $t_{2 g}$ and $e_{g}$ states.

The CoG was calculated for the $t_{2 g}$-like and $e_{g}$-like states, namely $\mathrm{CoG}\left(t_{2 g}\right)$ and $\operatorname{CoG}\left(e_{g}\right)$. Their difference $\Delta \mathrm{CoG}=\mathrm{CoG}\left(e_{g}\right)-\operatorname{CoG}\left(t_{2 g}\right)$ gives a measure of the state's shift introduced by the corresponding defect and charge state (species). Using $\Delta \mathrm{CoG}$ of the different species, a relative shift of the $\operatorname{CoG}\left(e_{g}\right)$ with respect to the undoped material was calculated via

$$
\text { relative } \operatorname{CoG}\left(e_{g}\right) \text { shift }=\Delta \operatorname{CoG}(\text { species })-\Delta \operatorname{CoG}(\text { undoped })
$$

This relative shift is in principle comparable to the one obtained by the EELS measurements, cf. 3.3.3.1.

The calculations performed used a projector augmented wave method within the CP-PAW code [121]. The local hybrid density functional PBE0r [122] was used. A supercell of 72 atoms $(2 \times 2 \times 3)$ plus the corresponding defect was simulated. All atomic positions were relaxed without symmetry constraints.

A comprehensive explanation of the calculation will be given in the doctoral thesis of Mohsen Sotoudeh [123] and in a publication of M. Sotoudeh, M. Bongers, V. Roddatis, J. Čížek, C. Nowak, M. Wenderoth, P. Blöchl and A. Pundt.

The DFT calculations and the interpretation of the in situ EELS measurements based on them were treated in a collaborated effort. The following statement clarifies the individual contributions to this collaboration: The DFT calculations of rutile and defects have been performed and evaluated by M. Sotoudeh. The resulting electronic structure of the defects has been explored by M. Sotoudeh and P. Blöchl. The thermodynamic calculation of the defect concentrations has been done by P. Blöchl using the energies obtained by M. Sotoudeh. The in situ EELS investigations and their evaluation have been carried out by Marian Bongers. The results of the EELS measurements and of the DFT calculations were interpreted in a joint collaboration by M. Bongers, M. Sotoudeh, A. Pundt, M. Wenderoth and P. Blöchl.

\footnotetext{
${ }^{25}$ These were for Ti and $\mathrm{O}$ the $s, p$ and $d$ orbitals.

${ }^{26}$ Examples are the $\mathrm{O} p$ orbital or the Ti $d_{x^{2}-y^{2}}$ and $d_{z^{2}}$ orbitals for the $t_{2 g}$ states.
} 


\section{Results}

This chapter presents the produced $\mathrm{Pd} / \mathrm{r}-\mathrm{TiO}_{2}$ system and the results obtained on it in a hydrogen gas environment.

The produced $\mathrm{Pd} / \mathrm{r}-\mathrm{TiO}_{2}$ samples are characterized in chap. 4.1 with respect to the orientation relationship of $\mathrm{Pd}$ on the $\mathrm{r}-\mathrm{TiO}_{2}$ crystals and to the interface condition. Chap. 4.3 presents the local in situ EELS experiments in hydrogen atmosphere that were performed on these $\mathrm{Pd} / \mathrm{r}-\mathrm{TiO}_{2}$ samples. DFT calculations of the hydrogen-r- $\mathrm{TiO}_{2}$ system with focus in the hydrogen related changes in the DoS are shown in chap. 4.4. The EELS results are compared to the DFT calculations in chap. 4.5 which allowed a rough estimate of the defect concentration in the close vicinity of the $\mathrm{Pd} / \mathrm{r}-\mathrm{TiO}_{2}$ interface. Microstructural changes that were observed after the hydrogen loading close to the $\mathrm{Pd} / \mathrm{r}-\mathrm{TiO}_{2}$ interface in $\mathrm{r}-\mathrm{TiO}_{2}$ are presented in chap. 4.6. VEPAS measurements carried out in hydrogen atmosphere allowed to get additionally insides in the presence of $\mathrm{V}_{\mathrm{Ti}}$ in $\mathrm{r}-\mathrm{TiO}_{2}$ and in changes observed in the Pd. These measurements are presented in chap. 4.7. Chap. 4.8 presents complementary ETEM investigations on hydrogen related changes of $\mathrm{Pd}$.

\section{1. $\mathrm{Pd} / \mathrm{r}-\mathrm{TiO}_{2}$ sample preparation and characterization of the initial state}

The first step for performing suitable experiments was the optimization of the $\mathrm{Pd} / \mathrm{r}$ $\mathrm{TiO}_{2}$ sample system with respect to crystallinity and to interface sharpness. This was a necessary requirement for the EELS experiments. This goal was reached by using a suitable $\mathrm{r}-\mathrm{TiO}_{2}$ crystal pretreatment (cf. chap. 3.1.1) and appropriate parameters for the magnetron sputter deposition used (cf. chap. 3.1.2). The influence of the pretreatment on the $\mathrm{r}-\mathrm{TiO}_{2}$ crystal surfaces is shown in chap. 4.1.1. The sputter deposited $\mathrm{Pd}$ films were characterized with respect to the orientation relationship and the interface condition which is shown in chap. 4.1.2.

\subsubsection{The $\mathrm{r}-\mathrm{TiO}_{2}$ crystal surface}

The results that are presented in this section were obtained in collaboration with Niklas Herwig [93].

The initial (as delivered) crystal surface was investigated with AFM, fig. 4.1a. In the 3D AFM height profile surface, steps can be recognized, but also a 'rough' and 


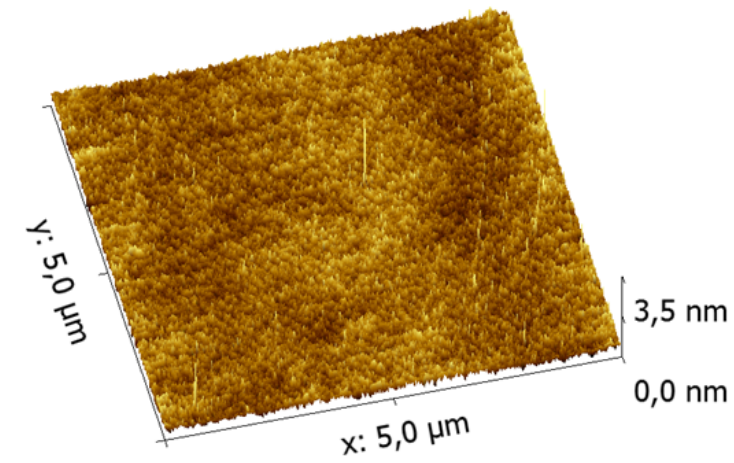

(a) The initial surface steps can be recognized, though the surface appears 'rough' and 'diffuse'.

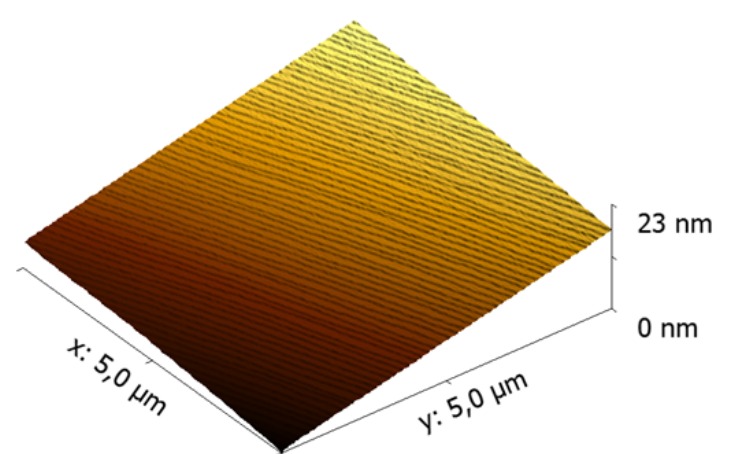

(b) After pretreatment the surface is clean and the surface steps are clearly visible.

Figure 4.1.: $25 \mathrm{\mu m}^{2}$ 3D AFM height profile of the $\mathrm{r}-\mathrm{TiO}_{2}$ crysal surface. In collaboration with Niklas Herwig [93].

'diffuse' crystal surface is visible. Some additional particles were present. After the pretreatment, the surface was flat and revealed a typical step structure shown in the 3D AFM height profile in fig. 4.1b. The $\mathrm{r}-\mathrm{TiO}_{2}$ crystal is clean after the pretreatment which is necessary for the production of a clean and sharp interface to the Pd film.

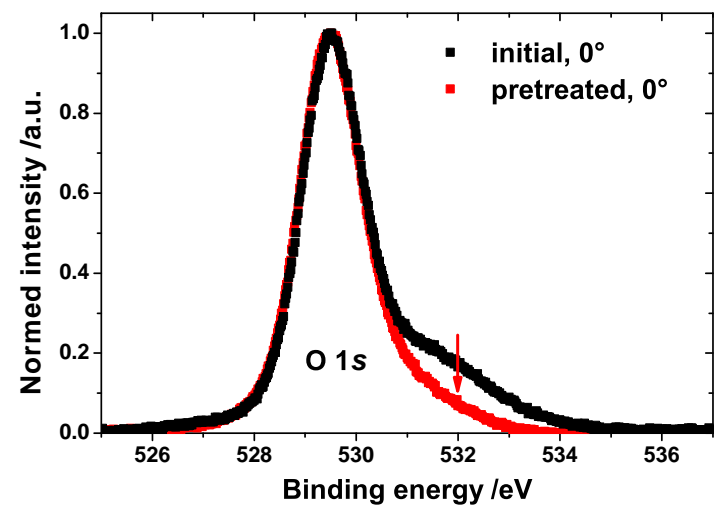

(a)

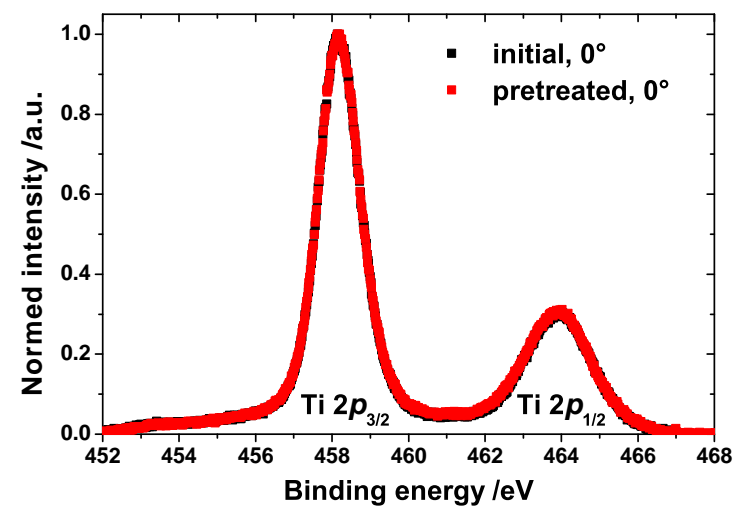

(b)

Figure 4.2.: X-ray photoelectron spectra of the $\mathrm{r}-\mathrm{TiO}_{2}$ substrate surface at an incident angle of $0^{\circ}$. The oxygen $1 s$ edge (a) as well as the Ti $2 p$ edge (b) are shown before and after the pretreatment. The oxygen $1 s$ edge has a shoulder which decreased after the pretreatment. In collaboration with Julius Scholz and Niklas Herwig [93].

The crystal surface cleanliness after the pretreatment was also investigated by XPS measurements. An XP spectrum of a $\mathrm{r}-\mathrm{TiO}_{2}$ substrate surface is shown in fig. 4.2. Here, the oxygen $1 s$ as well as the Ti $2 p$ XP spectra are depicted for before and after 
the pretreatment in fig. $4.2 \mathrm{a}$ and $4.2 \mathrm{~b}$, respectively. The oxygen $1 s$ peak as well as the Ti $2 p$ peak do not change due to the pretreatment and resemble the expected spectrum described in literature $[124,125]$. This confirms a good quality in the initial state. The oxygen $1 s$ peak exhibits a shoulder that decreases strongly after the pretreatment. This indicates a decreasing amount of surface species (e.g. C-O, O-H, H-O-H) [126] and therefore a cleaner surface after the pretreatment.

In summary, with the applied pretreatment, containing HF etching and subsequent thermal annealing in $\mathrm{O}$, the $\mathrm{r}-\mathrm{TiO}_{2}$ crystals revealed a clean surface with clearly visible and well-separated surface steps.

\subsubsection{Pd films on pretreated $\mathrm{r}-\mathrm{TiO}_{2}$ crystals}

\begin{tabular}{lccc}
\hline \hline deposition parameter & $16.4 \mathrm{~nm}$ & $28.0 \mathrm{~nm}$ & $\sim 200 \mathrm{~nm}$ \\
\hline$p_{\mathrm{Ar}} / \mathrm{Pa}$ & $2.4 \cdot 10^{-1}$ & $2.4 \cdot 10^{-1}$ & $2.4 \cdot 10^{-1}$ \\
$T_{\mathrm{D}} / \mathrm{K}$ & $\mathrm{RT}$ & $\mathrm{RT}$ & 1023 \\
$p_{\mathrm{O}_{2}} / \mathrm{Pa}$ & - & - & $7 \cdot 10^{-3}$ \\
$P_{\mathrm{D}} / \mathrm{W}$ & 10 & 10 & 20 \\
$r_{\mathrm{D}} / \mathrm{nm} \mathrm{min}$ & -1 & 4 & 11.8 \\
\hline property & 4 & & $(111)$ \\
\hline$(h k l)_{\text {normal }}$ & $(111)$ & $\mathrm{nc}$ & $\begin{array}{c}\text { textured with two } \\
d_{111} / \AA\end{array}$ \\
crystallinity & $(2.2395 \pm 0.0003)$ & $(2.2463 \pm 0.0006)$ & preferred orientations \\
& $\mathrm{nc}$ & & - \\
$\mathrm{RMS} / \mathrm{nm}$ & 0.2 & 0.2 & \\
\hline \hline
\end{tabular}

Table 4.1.: Deposition parameters and the resulting properties are shown for the different $\mathrm{Pd}$ films. Here $T_{\mathrm{D}}$ is the deposition temperature, $p_{\mathrm{O}_{2}}$ is the oxygen and $p_{\text {Ar }}$ is the Ar partial pressure, $P_{\mathrm{D}}$ is the power for the sputter deposition, $r_{\mathrm{D}}$ is the corresponding sputter rate, $(h k l)_{\text {normal }}$ are the via XRD observed $\mathrm{Pd}$ orientations in normal direction, $d_{111}$ are the (111) lattice plane distances in normal direction and RMS is the root mean square roughness. 'nc' means nanocrystalline.

Two types of $\mathrm{Pd}(111)$ films were produced with magnetron sputtering on the pretreated $\mathrm{r}-\mathrm{TiO}_{2}(110)$ crystals. The first type were a couple of $100 \mathrm{~nm}$ thick Pd films deposited at $1023 \mathrm{~K}$ where the focus lies on high crystallinity and low defect concentration both in the $\mathrm{Pd}$ film and in the $\mathrm{r}-\mathrm{TiO}_{2}$ substrate. These films were used for the in situ EELS measurements. The second type were thin Pd film in the range of $15 \mathrm{~nm}$ to $30 \mathrm{~nm}$ deposited at RT. Here, the focus lies on a flat and closed Pd surface. These films reveal a nanocrystalline structure with the possibility of a high lateral stress. The latter Pd films are not the focus, and are characterized in appendix A.2. 
The deposition of the $\sim 100 \mathrm{~nm}$ Pd films was carried out at elevated temperatures (cf. tab. 4.1). At this temperature, the grain size increased in comparison to the thin Pd film but solid dewetting occurred in the Pd films. Dewetting and an increasing grain size of thin Pd films, with increasing deposition temperature was shown in a collaboration with Maximilian Litschauer [127]. Hence, thick films were needed to get (almost) closed $\mathrm{Pd}$ layers at $1023 \mathrm{~K}$, this is the reason for the chosen film thickness of a couple of $100 \mathrm{~nm}$.

A summary of XRD, XRR, texture, AFM and TEM investigations on the Pd films is given together with the used sputter deposition conditions in tab. 4.1.

\subsubsection{Thick Pd films prepared at $1023 \mathrm{~K}$}

The $\sim 100 \mathrm{~nm}$ Pd films were investigated with XRD, texture and TEM measurements presented in the following.

XRD investigations were performed on the thicker Pd films grown on $\mathrm{r}-\mathrm{TiO}_{2}(110)$. The diffractogram for an approximately $200 \mathrm{~nm} \mathrm{Pd}$ film is shown in fig. 4.3. The

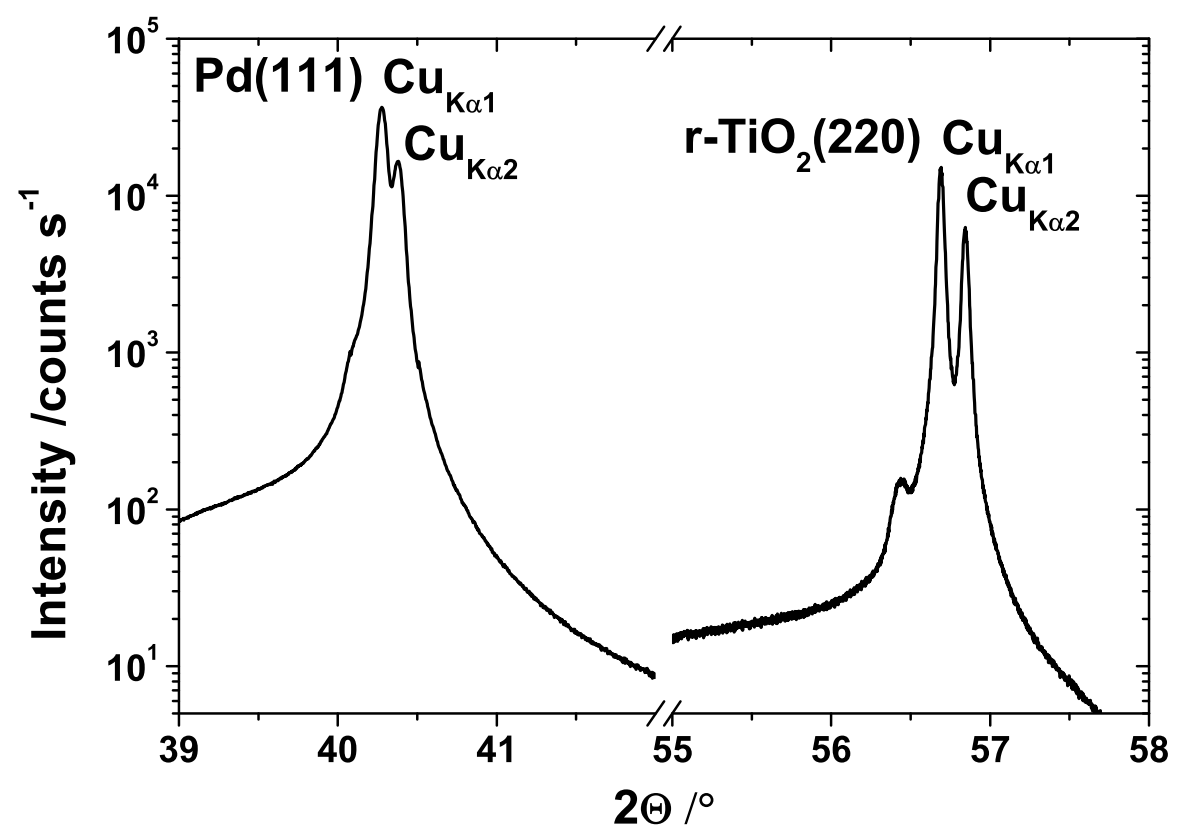

Figure 4.3.: XR diffractogram showing the measured intensity versus $2 \Theta$ of a $200 \mathrm{~nm}$ thick $\mathrm{Pd}$ film deposited at $1023 \mathrm{~K}$. The selected angle range shows the $\mathrm{Pd}(111)$ and the $\mathrm{r}-\mathrm{TiO}_{2}(220)$ interferences for the $\mathrm{Cu} K_{\alpha_{1}}$ and $K_{\alpha_{2}}$ line.

diffractogram shows interferences from $\mathrm{Pd}(111)$ and $\mathrm{r}-\mathrm{TiO}_{2}(220)$. No other $\mathrm{Pd}$ orientations were observed. The maximum of the $\operatorname{Pd}(111) \mathrm{Cu} K_{\alpha_{1}}$ interference is observed 
at $2 \Theta=(40.2150 \pm 0.0003)^{\circ}$ which corresponds to $d_{111}=(2.24066 \pm 0.00002) \AA$. Consequently, the $\mathrm{Pd}$ lattice is contracted in the normal direction by approximately $\Delta d_{111}=-(0.0054 \pm 0.0004) \AA$ when compared to the bulk value from Arblaster [72]. This corresponds to a strain $\epsilon_{111}=-(0.0024 \pm 0.0002)$ in the normal direction. Due to the anisotropic $\mathrm{r}-\mathrm{TiO}_{2}(110)$ surface, the lateral stress in the Pd film cannot be calculated from the compression in the normal direction, cf. chap. 2.3.1. Though it is expected that the lateral directions reveals an expansion and thus a tensile stress state.

Pole figure measurements were performed on a $200 \mathrm{~nm} \mathrm{Pd}$ film to investigate the growth behavior of $\mathrm{Pd}$ on the $\mathrm{r}-\mathrm{TiO}_{2}$ substrate at elevated temperatures. The resulting $\operatorname{Pd}(111)$ pole figure and its simulation are shown in fig. 4.4. The central (111)

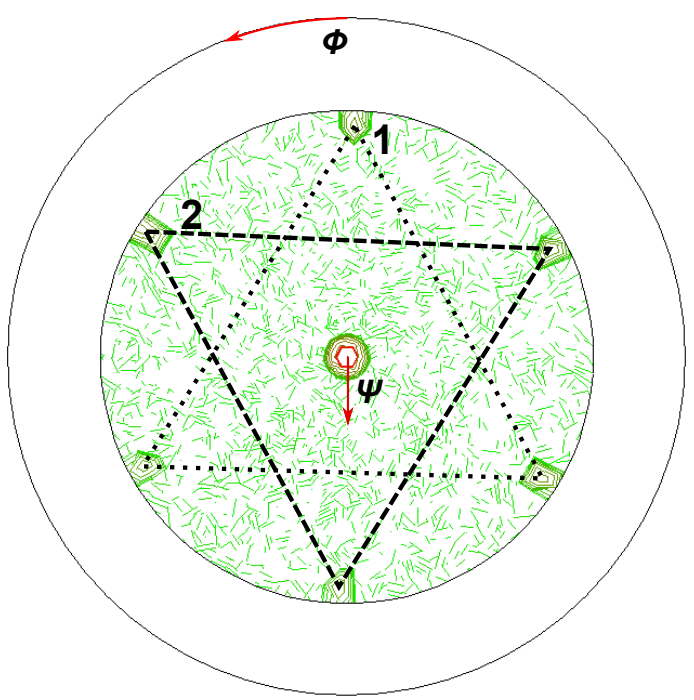

(a) (111) pole figure of a $200 \mathrm{~nm}$ Pd film deposited at $1023 \mathrm{~K}$. Two preferred (111) orientations are visible and indicated each with a triangle and a number. Measurement displayed in logarithmic scale.

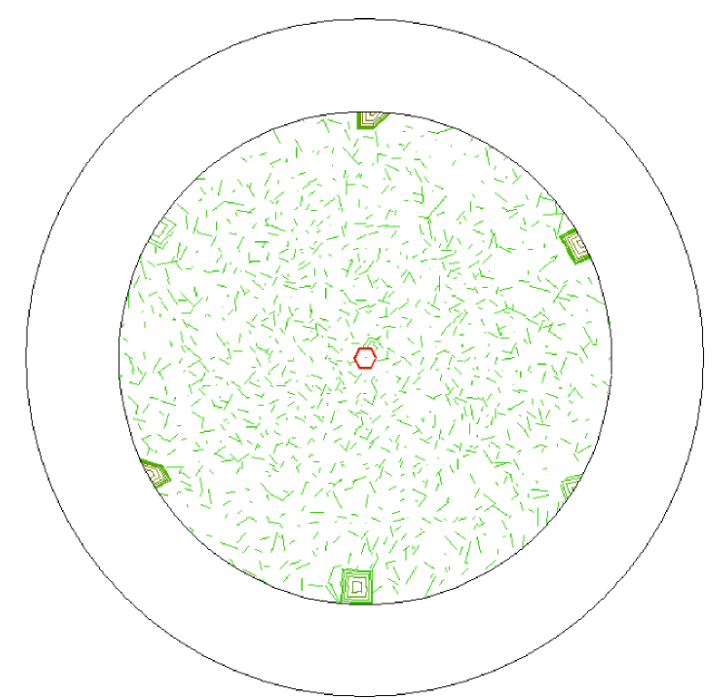

(b) MulTex simulation of the (111) pole figure with three components: two textured and one isotropic.

Figure 4.4.: Pole figure measurement showing (a) the $\operatorname{Pd}(111)$ pole figure and (b) the corresponding simulation. The outer circle corresponds to $\Psi=90^{\circ}$, the circle further inside corresponds to $\Psi=72^{\circ}$ which was the chosen limit.

reflection is surrounded by six reflections at $70.5^{\circ}$. These reflections correspond to the main reflection and reveal existing preferred orientations in the Pd film. Each of the three reflections are at an angle of $109.5^{\circ}$ with respect to each other, and thus correspond to the same orientation. Two preferred orientations are therefore observed and marked with the two triangles in fig. 4.4a. A simulation of the pole figure is 
done via MulTex and shown in fig. 4.4b. The pole figure fit is performed with three components: two texture components ( 1 and 2 ) that correspond to the two mentioned preferred orientations and one isotropic component that considers the background of the pole figure measurement. Component (/orientation) 1 has an approximate Euler angle $g=\left(357^{\circ}, 55^{\circ}, 45^{\circ}\right)$. Component 2 has an approximate Euler angle $g=\left(298^{\circ}\right.$, $53^{\circ}, 46^{\circ}$ ). The background visible in the pole figure is considered as an isotropic component. Note, an additional weak third orientation can be found: An additional reflection is visible using an exponential scale for the $\operatorname{Pd}(111)$ pole figure as shown in the appendix in fig. A.6. The reflection is tilted away from the center by $20^{\circ}$ indicating a third orientation in the $\mathrm{Pd}$ film. A fit is not possible since the intensity is low and no corresponding reflections occurred. The volume fraction of this component can be considered as insignificant. The simulation gives the resulting orientation distribution function (ODF) of the two fit components ${ }^{1}$, which is shown in the appendix in fig. A.7.

The simultaneously measured $\mathrm{r}-\mathrm{TiO}_{2}(110)$ pole figure (not shown) did show the main reflection in the center but no additional reflections. This was probably due to the strong absorption of the overlying Pd film. Though, to study the orientation relationship between $\mathrm{Pd}$ and $\mathrm{r}-\mathrm{TiO}_{2}$ the two preferred orientations need to be aligned to the orientation of the substrate. This was done using $\phi$ scans.

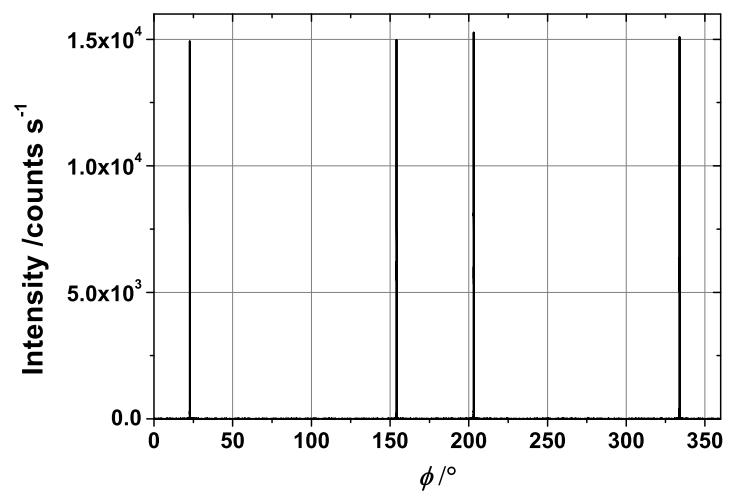

(a) $\psi=38.5526^{\circ}$, ideally $\psi=38.81^{\circ}$.

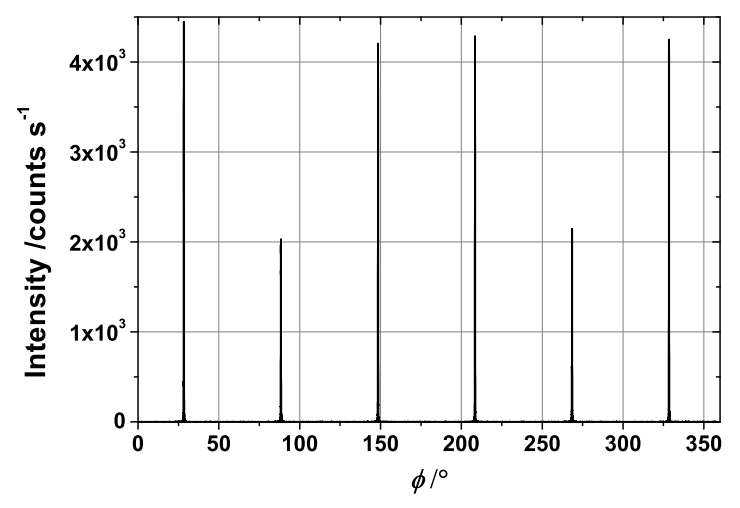

(b) $\psi=70.102^{\circ}$, ideally $\psi=70.53^{\circ}$.

Figure 4.5.: $\phi$ scans of the (a) $\mathrm{r}^{-\mathrm{TiO}_{2}}(211)$ and (b) $\mathrm{Pd}(111)$ interference.

$\phi$ scans were carried out to relate the $\mathrm{Pd}$ growth on the $\mathrm{r}-\mathrm{TiO}_{2}(110)$ crystals. In comparison to the pole figure measurement, the sample was mounted differently and therefore the $\phi$ angles did not coincide with the ones from the $\operatorname{Pd}(111)$ pole figure (cf. fig 4.4). $\phi$ scans were done on the $\mathrm{Pd}(111)$ and $\mathrm{r}-\mathrm{TiO}_{2}(211)$ interference on the same $200 \mathrm{~nm} \mathrm{Pd}$ film as before and shown in fig. 4.5.

\footnotetext{
${ }^{1}$ The simulation includes also the $\operatorname{Pd}(200)$ and $\operatorname{Pd}(220)$ pole figure. They do not contain additional information and therefore are not shown.
} 
As expected for the $\mathrm{r}-\mathrm{TiO}_{2}(110)$ single crystal, the $\phi$ scan at $\psi=38.5526^{\circ}$ shows four sharp (211) interferences, cf. fig. 4.5a. The $\phi$ scan at $\psi=70.102^{\circ}$ for the $\operatorname{Pd}(111)$ interference shows six sharp maxima, as shown in fig. $4.5 \mathrm{~b}$, which correspond to the 6 poles in the $\operatorname{Pd}(111)$ pole figure (cf. fig. 4.4a). The corresponding maxima positions are summarized in tab. 4.2 .

\begin{tabular}{llrr}
\hline \hline interference & $\phi /^{\circ}$ & $\Delta \phi /^{\circ}$ & expected $\Delta \phi /^{\circ}$ \\
\hline${\mathrm{r}-\mathrm{TiO}_{2}(211)}^{22.7848 \pm 0.0004}$ & 48.9018 & 49.00 \\
& $154.111 \pm 0.002$ & 131.3262 & 131.03 \\
& $203.120 \pm 0.002$ & 49.009 & 49.00 \\
& $333.883 \pm 0.003$ & 130.763 & 131.03 \\
\hline $\mathrm{Pd}(111)$ component 1 & $28.3266 \pm 0.0002$ & 119.8858 & 120 \\
& $148.4523 \pm 0.0005$ & 120.1257 & 120 \\
& $268.4408 \pm 0.002$ & 119.9885 & 120 \\
$\mathrm{Pd}(111)$ component 2 & $88.3754 \pm 0.0007$ & 119.9561 & 120 \\
& $208.4242 \pm 0.0007$ & 120.0488 & 120 \\
& $328.4193 \pm 0.002$ & 119.9951 & 120 \\
\hline \hline
\end{tabular}

Table 4.2.: Maxima of the measured interferences of the $\phi$ scans from fig. 4.5.

\begin{tabular}{llrr}
\hline \hline interference & $\phi /^{\circ}$ & $\Delta \phi /^{\circ}$ & expected $\Delta \phi /^{\circ}$ \\
\hline${\mathrm{r}-\mathrm{TiO}_{2}(211)}^{24.4509}$ & 48.9018 & 49.00 \\
& 155.7771 & 131.3262 & 131.03 \\
& 204.7861 & 49.009 & 49.00 \\
& 335.5491 & 130.763 & 131.03 \\
\hline $\mathrm{Pd}(111)$ component 1 & 29.9927 & 119.8858 & 120 \\
& 150.1184 & 120.1257 & 120 \\
& 270.1069 & 119.9885 & 120 \\
$\mathrm{Pd}(111)$ component 2 & 90.0415 & 119.9561 & 120 \\
& 210.0903 & 120.0488 & 120 \\
& 330.0854 & 119.9951 & 120 \\
\hline \hline
\end{tabular}

Table 4.3.: Aligned $\phi$ angles of the measured interference maxima from fig. 4.5. The $\phi$ angles are aligned that the $\mathrm{r}-\mathrm{TiO}_{2}$ single crystal substrate has its $(00 \overline{1})$ planes at $\phi=0^{\circ}$ and its $(\overline{1} 10)$ plane at $\phi=270^{\circ}$.

The $\phi$ scan of the $\mathrm{r}-\mathrm{TiO}_{2}(211)$ interferences enables the alignment of the $\phi$ axis to the coordinate system of the $\mathrm{r}-\mathrm{TiO}_{2}$ single crystal. A tilt in the $\phi$ angle by $+1.6661^{\circ}$ aligns the $\phi$ axis to the $\mathrm{r}-\mathrm{TiO}_{2}$ crystal in a way that $\phi=0^{\circ}$ corresponds to a $\mathrm{r}-\mathrm{TiO}_{2}\{001\}$ 
plane and $\phi=90^{\circ}$ corresponds to $\mathrm{r}-\mathrm{TiO}_{2}\{\overline{1} 10\}$ plane. The offset of $+1.6661^{\circ}$ was also applied on the $\phi$ angles of the measured $\operatorname{Pd}(111)$ interferences. The resulting aligned $\phi$ angles for $\mathrm{r}-\mathrm{TiO}_{2}(211)$ and $\mathrm{Pd}(111)$ are shown in tab. 4.3 .

Thus, $\phi=0^{\circ}$ corresponds to a $\operatorname{Pd}\{01 \overline{1}\}$ plane for component 1 and to a $\operatorname{Pd}\{\overline{1} 10\}$ plane for component 2. $\phi=90^{\circ}$ corresponds to a $\operatorname{Pd}\{2 \overline{1} \overline{1}\}$ plane for component 1 and to a $\operatorname{Pd}\{12 \overline{2}\}$ plane for component 2. For each of the $\phi$ angles, both components reveal the same plane family and thus have the same orientation relationship on $\mathrm{r}$ $\mathrm{TiO}_{2}$. Therefore the orientation relationship of $\mathrm{Pd}$ is found to be

$$
[2 \overline{1} \overline{1}](111) \|[1 \overline{1} 0](110) \mathrm{r}_{-} \mathrm{TiO}_{2}
$$

and

$$
[01 \overline{1}](111) \|[00 \overline{1}](110) \mathrm{r}-\mathrm{TiO}_{2} \text {. }
$$

The orientation relationship is depicted in fig. 4.6.

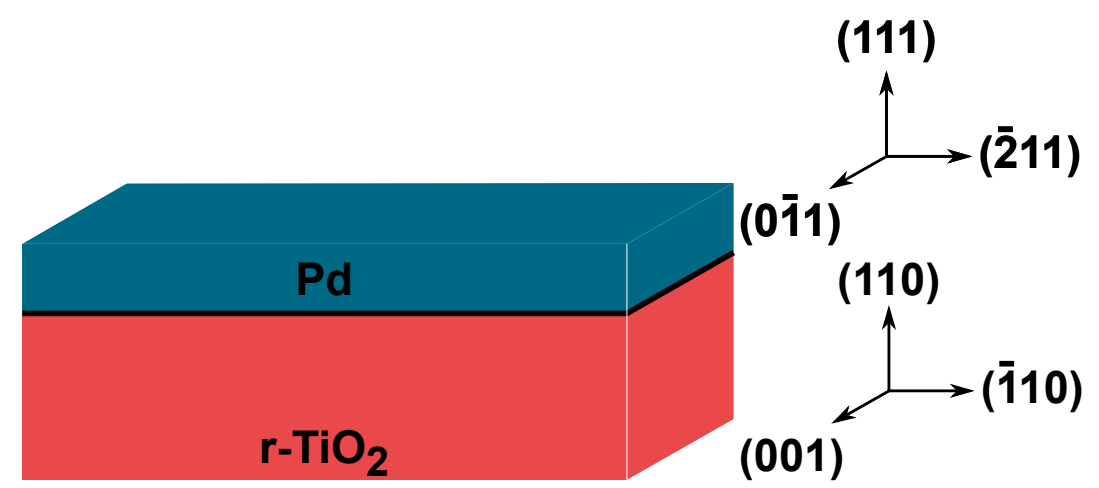

Figure 4.6.: The orientation relationship of the $200 \mathrm{~nm} \mathrm{Pd}$ films deposited at $1023 \mathrm{~K}$ on a $\mathrm{r}-\mathrm{TiO}_{2}(110)$ single crystal is sketched. Coloring after CPK coloring of 'Jmol' [14].

TEM lamella were prepared from the thick $\mathrm{Pd}$ films on $\mathrm{r}-\mathrm{TiO}_{2}$, cf. chap. 3.2.1. The in situ EELS experiments were carried on this lamella, therefore the structure of the $\mathrm{Pd} / \mathrm{r}-\mathrm{TiO}_{2}$ lamella (before the hydrogen loading) is of interest and discussed in the following.

The pretreatment and chosen deposition parameters for the $\mathrm{Pd} / \mathrm{r}-\mathrm{TiO}_{2}$ samples (cf. chap. 3.1 and 4.1.1) as well as the TEM lamella preparation yielded to a $\mathrm{Pd} / \mathrm{r}-\mathrm{TiO}_{2}$ structure which is shown in fig. 4.7. An interface is found with a sharpness of one to two monolayers (corresponding to $0.325 \mathrm{~nm}$ to $0.650 \mathrm{~nm}$ ), cf. fig. 4.7a. According to fig. 4.7b every third to fourth $\mathrm{Pd}(\overline{1} 11)$ plane ends in between and not on top of a $\mathrm{Ti}$ row of the $\mathrm{r}-\mathrm{TiO}_{2}$ as indicated with the green dashed lines. These interjectional $\mathrm{Pd}$ half planes are dislocations, thus a semi-coherent interface has grown between $\mathrm{Pd}$ and $\mathrm{r}-\mathrm{TiO}_{2}$ in the presented direction. For the selected area, the orientation relationship of $[01 \overline{1}](111) \|[00 \overline{1}](110) \mathrm{r}-\mathrm{TiO}_{2}$ and $[2 \overline{1} \overline{1}](111) \|[1 \overline{1} 0](110) \mathrm{r}-\mathrm{TiO}_{2}$ is found. This is in 


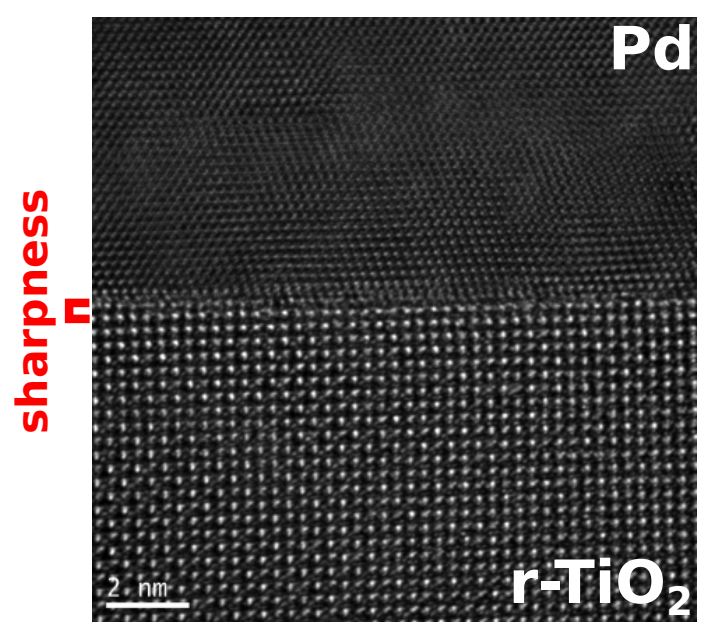

(a)

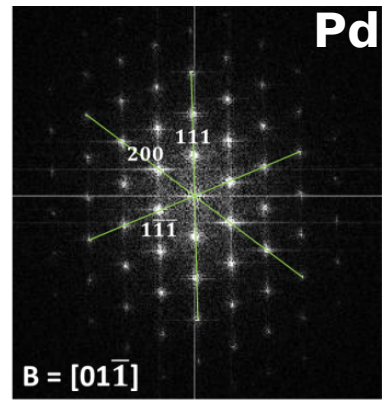

(c)

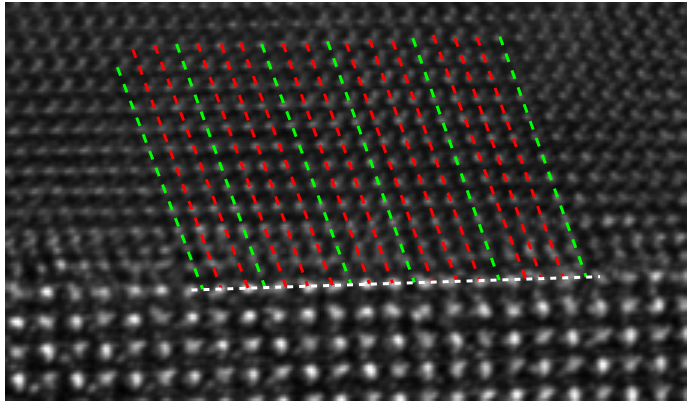

(b)

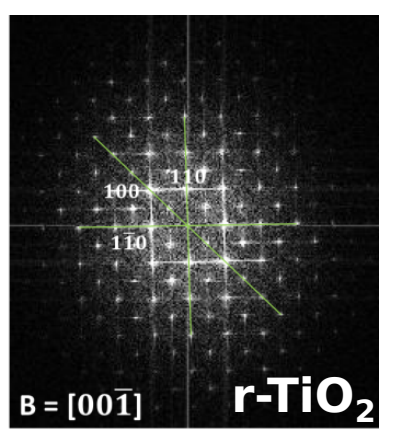

(d)

Figure 4.7.: A HRTEM picture of a prepared $\mathrm{Pd} / \mathrm{r}-\mathrm{TiO}_{2}$ interface region is shown in (a). A sharp interface between one to two monolayers is revealed and no defects, with a dimension of one or higher, can be found in the $\mathrm{r}-\mathrm{TiO}_{2}$ interface region. A zoom of (a) is shown in (b). Here, $\mathrm{Pd}(\overline{1} 11)$ half planes ending at Ti rows of the $\mathrm{r}-\mathrm{TiO}_{2}$ are indicated with red dashed lines. $\operatorname{Pd}(\overline{1} 11)$ half planes that end in between Ti rows are indicated with green dashed lines. The corresponding fast Fourier transform of the Pd (c) and the $\mathrm{r}-\mathrm{TiO}_{2}$ (d) part confirm the high crystallinity and allow for indexing the lattice directions as indicated in the corresponding subfigures. The B vectors indicate the direction into the paper plane.

line with the results of the pole figure measurement and $\phi$ scans and confirms the orientation relationship, cf. fig. 4.6.

In summary, the $200 \mathrm{~nm}$ thick Pd films deposited at $1023 \mathrm{~K}$ reveal a crystalline structure with two preferred $\operatorname{Pd}(111)$ orientations. A orientation relationship of $[\overline{2} 11](111) \|$ [110](110) $\mathrm{r}^{-\mathrm{TiO}_{2}}$ is observed for both orientations with XRD and HRTEM investigations and depicted in fig. 4.6. XRD measurements reveal a compression in normal direction of $0.24 \%$. 


\section{2. $I-V$ characterization of hydrogen loaded $\mathrm{Pd} / \mathrm{r}-\mathrm{TiO}_{2}$}

A proof-of-concept study was performed for the produced $\mathrm{Pd} / \mathrm{r}-\mathrm{TiO}_{2}$ system on its ability to sense hydrogen (cf. chap. 2.3.2.1). This was done in collaboration with Jorge de Heuvel [128]. Current-voltage characteristics were taken on a stack of $\mathrm{Pd} / \mathrm{r}$ $\mathrm{TiO}_{2}(110) / \mathrm{Ti} / \mathrm{Pd}$. The stack was prepared with magnetron sputtering deposition at RT and had the dimensions: $1 \mu \mathrm{m} \mathrm{Pd} / 500 \mu \mathrm{m} \mathrm{r}-\mathrm{TiO}_{2} / 0.8 \mu \mathrm{m} \mathrm{Ti} / 1 \mu \mathrm{m} \mathrm{Pd}$. In this stack, a Schottky barrier is established at the $\mathrm{Pd} / \mathrm{r}-\mathrm{TiO}_{2}$ contact (cf. chap. 2.3.2) while the $\mathrm{r}-\mathrm{TiO}_{2} / \mathrm{Ti}$ back-contact was ohmic. The additional $\mathrm{Pd}$ film on the backside Ti prevents oxidation. Thus, a typical metal/semiconductor characteristic can be measured through this stack. Though the resistivity of the $\mathrm{r}-\mathrm{TiO}_{2}$ crystal itself was originally high, therefore it was reduced by annealing in high vacuum $\left(\approx 10^{-4} \mathrm{~Pa}\right)$ at $773 \mathrm{~K}$ (color change to slightly bluish) [128, 129].

These stacked contacts were loaded with hydrogen in a high vacuum chamber. The sample was kept for $70 \mathrm{~min}$ in a hydrogen pressure of $14.3 \mathrm{~Pa}$. Subsequently the sample was transferred in ambient conditions to an Agilent 4155C Parameter Analyser (IV. Physics Institute, in collaboration with Jörg Malindretos) and current-voltage characteristics were taken as a function of time $t . t$ here describes the time after hydrogen loading that the sample was kept in ambient conditions. These characteristics are displayed in fig. 4.8. The first characteristic taken at $2 \mathrm{~min} 30 \mathrm{~s}$ (red) revealed a clear increase in conductivity in comparison to the initial state (black). Over time the conductivity decreased and the sample converged back to its initial characteristics. This is a typical gas sensing behavior (cf. chap. 2.3.2.1) and can be compared qualitatively to fig. 2.16 .

The general shape of the characteristics differs from the $\mathrm{Pd} / \mathrm{r}-\mathrm{TiO}_{2}$ system studied by Yamamoto et al. [10], as presented in fig. 2.16, which is a strong current increase or typical diode behavior, appearing at higher voltages. It was found that this difference is due to a poorly established ohmic back contact on the $\mathrm{Pd} / \mathrm{r}-\mathrm{TiO}_{2}(110) / \mathrm{Ti} / \mathrm{Pd}$ stack. The $\mathrm{r}-\mathrm{TiO}_{2}(110) / \mathrm{Ti}$ back contact revealed an additional diode behavior but switched in the opposite voltage direction. Thus, the flowing current is limited for the $\mathrm{Pd} / \mathrm{r}$ $\mathrm{TiO}_{2}(110)$ contact due to the $\mathrm{r}-\mathrm{TiO}_{2}(110) / \mathrm{Ti}$ back contact. However, the prepared $\mathrm{Pd} / \mathrm{r}-\mathrm{TiO}_{2}(110) / \mathrm{Ti} / \mathrm{Pd}$ stacks allowed for hydrogen gas sensing.

In summary, the produced $\mathrm{Pd} / \mathrm{r}-\mathrm{TiO}_{2}$ samples were capable of hydrogen gas sensing. While the system did not produce the best gas sensing performance, understanding the involved processes at the interface and its vicinity is the focus of this study. 


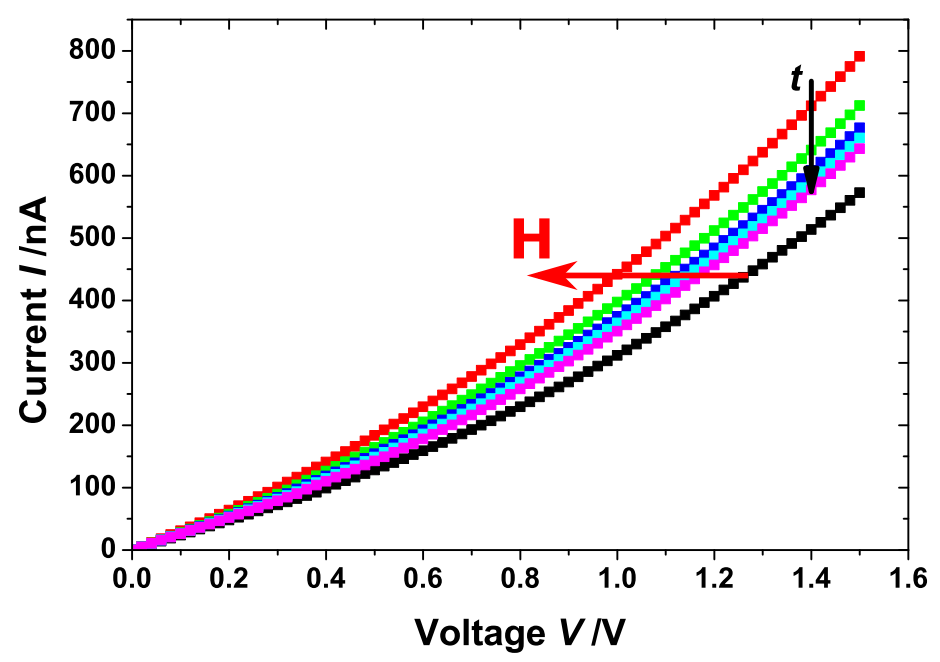

Figure 4.8.: Current-voltage characteristics of a $\mathrm{Pd} / \mathrm{r}-\mathrm{TiO}_{2} / \mathrm{Ti} / \mathrm{Pd}$ stacked sample prior loaded with $\mathrm{H}$. Each characteristic was measured in a certain time after the hydrogen loading in ambient conditions: $2 \mathrm{~min} 30 \mathrm{~s}$ (red), $6 \mathrm{~min} 30 \mathrm{~s}$ (green), $10 \mathrm{~min}$ $10 \mathrm{~s}$ (blue), $14 \mathrm{~min}$ (cyan), $1 \mathrm{~h} 26 \mathrm{~min} 30 \mathrm{~s}$ (pink). The black characteristic represents the initial state before the loading. The hydrogen loading increased the flowing current $I$ at comparable voltages $V$. Over time this effect became weaker which is a typical gas sensing behavior. In collaboration with Jorge de Heuvel [128].

\subsection{Local EELS on hydrogen loaded $\mathrm{Pd} / \mathrm{r}-\mathrm{TiO}_{2}$}

Changes in the Ti $L_{3,2}$ ELNES were studied as a function of the hydrogen gas pressure and of the distance from the $\mathrm{Pd} / \mathrm{r}-\mathrm{TiO}_{2}$ interface $z$ on a cross-section $\mathrm{Pd} / \mathrm{r}-\mathrm{TiO}_{2}$ TEM lamella. During the work, tens of thousands of single EEL spectra were acquired and evaluated; just a small selection is shown in this chapter. To acquire EEL spectra with sufficient energy resolution and stability a series of proceeding measurements was performed, described in chap. 3.3.1. Details about the experimental procedure are given in chap. 3.2.2 and 3.3.2.

Before discussing the EELS results, it is noted that all acquired EEL spectra resembled the typical $\mathrm{r}-\mathrm{TiO}_{2}$ fingerprint with the white lines $a, b / b^{\prime}, c$ and $d$ as shown in fig. 3.17 and described in chap. 2.1.2. At a hydrogen pressure of $10 \mathrm{~Pa}$, the Ti $L$ edge revealed changes in the $b / b^{\prime}$ and $d$ white line in the close vicinity of the interface. These changes were analyzed by means of energy splitting $\mathrm{d} E\left(b^{\prime}-a\right)$, using difference spectra for different $z$ and using the means of the center of gravity (CoG) of the $b / b^{\prime}$ white line $\left(\mathrm{CoG}\left(e_{g}\right)\right)$, fig. 4.9 and 4.10 .

As a reference, SI patterns were taken far away from the interface in the 'bulk' of the $\mathrm{r}-\mathrm{TiO}_{2}$ single crystal. This was done for all hydrogen pressures, though for simplicity $\mathrm{d} E\left(b^{\prime}-a\right)$ is only shown for before the hydrogen loading in fig. 4.9a and at $10 \mathrm{~Pa}$ hydrogen in fig. 4.9c. Both graphs show $\mathrm{d} E\left(b^{\prime}-a\right)$ against a relative position. 
The relative position has no meaning regarding the interface distance $z$; it appears just from the SI pattern applied in the bulk material. Thus it was expected that all values are the same within one measurement. Fig. 4.9 reveals that hydrogen has no

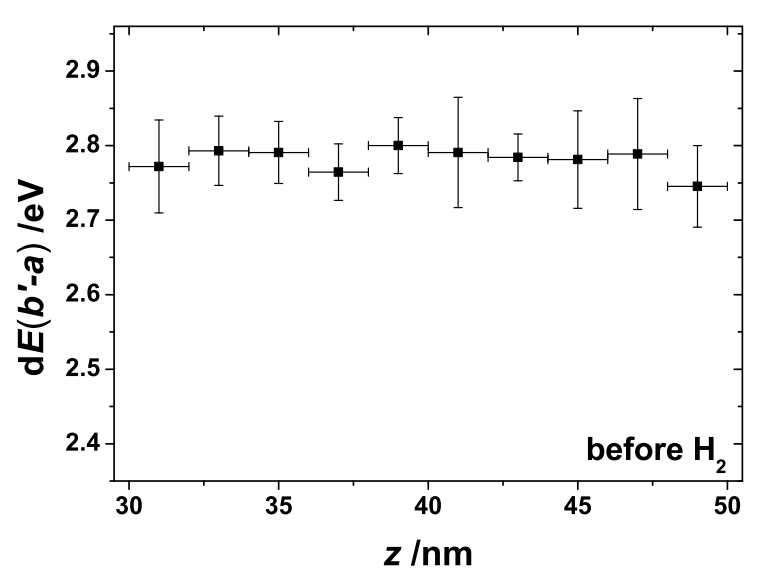

(a) About $30 \mathrm{~nm}$ away from the interface.

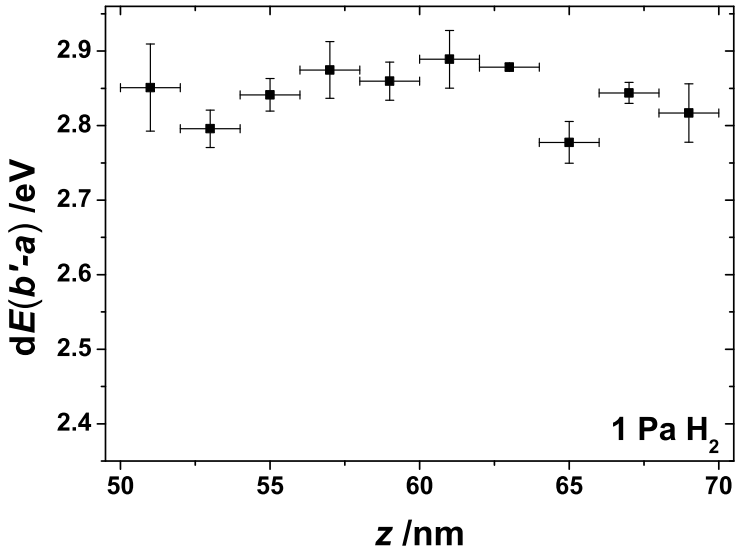

(b) About $50 \mathrm{~nm}$ away from the interface.

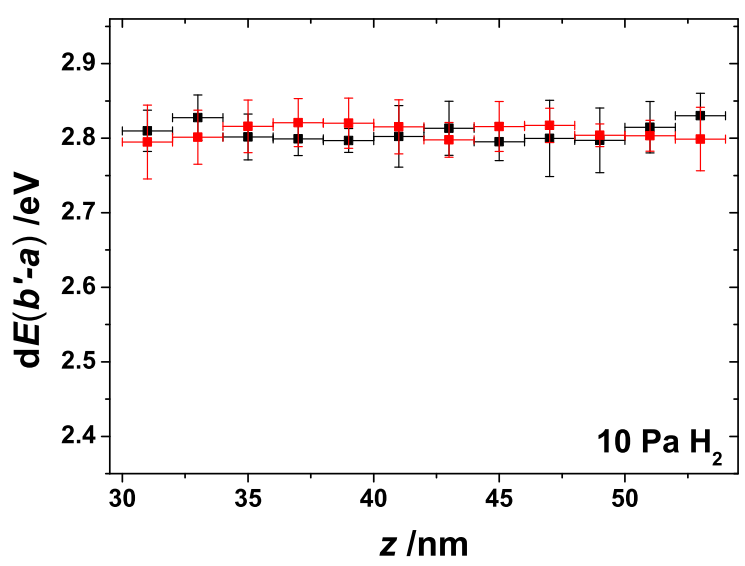

(c) About $30 \mathrm{~nm}$ away from the interface.

Figure 4.9.: Energy splitting $\mathrm{d} E\left(b^{\prime}-a\right)$ between the $b^{\prime}$ and $a$ white line in the $\mathrm{r}-\mathrm{TiO}_{2}$ far from the interface. The distance is not connected to the interface and thus has no physical meaning. (a) before hydrogen loading, (b) at $1 \mathrm{~Pa}$ and (c) at $10 \mathrm{~Pa}$ hydrogen gas pressure. For the latter, two measurements at different locations are shown. $\mathrm{d} E\left(b^{\prime}-a\right)$ in the 'bulk' $\mathrm{r}-\mathrm{TiO}_{2}$ was constant with $2.8 \mathrm{eV}$ at any studied hydrogen pressure. The bulk spectra were taken approximately $30 \mathrm{~nm}$ and more away from the interface in the $\mathrm{r}-\mathrm{TiO}_{2}$.

measurable influence on the $\mathrm{r}-\mathrm{TiO}_{2}$ single crystal (with the presented EELS method). The energy splitting $\mathrm{d} E\left(b^{\prime}-a\right)$ was constant at any applied hydrogen pressure, having a value of $2.8 \mathrm{eV}$. Since the relative position shown in fig. 4.9 has no physical meaning, a linear regression of each data set in fig. 4.9c (black and red) was applied (with 
OriginPro) and from both values the following weighted average ${ }^{2}$ was calculated

$$
\mathrm{d} E\left(b^{\prime}-a\right)_{\text {bulk r- } \mathrm{TiO}_{2}}=(2.808 \pm 0.005) \mathrm{eV} .
$$

The evaluated data which are shown in fig. 4.9 exhibit a scattering in the average

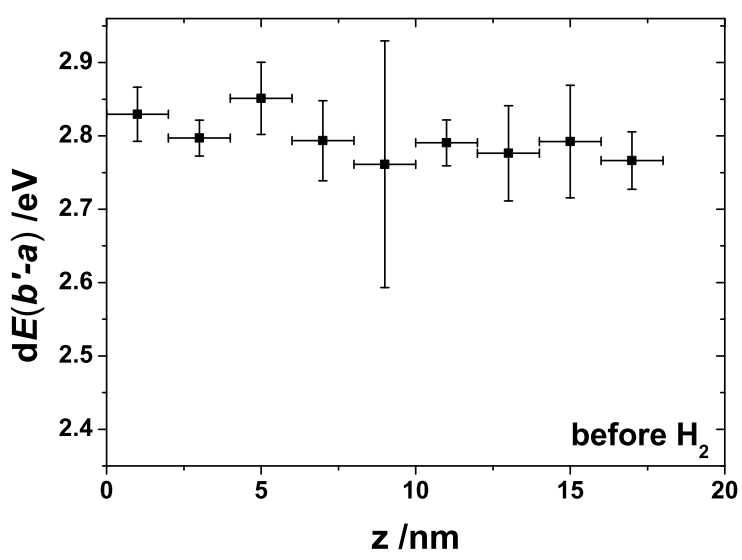

(a)

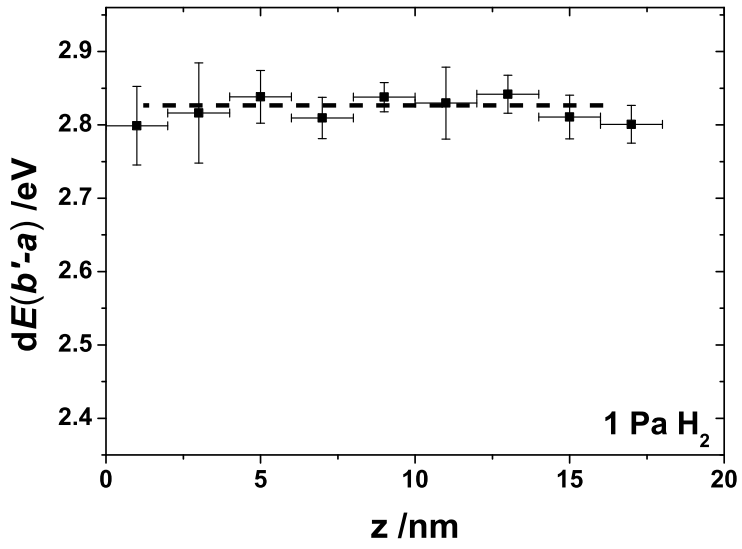

(b)

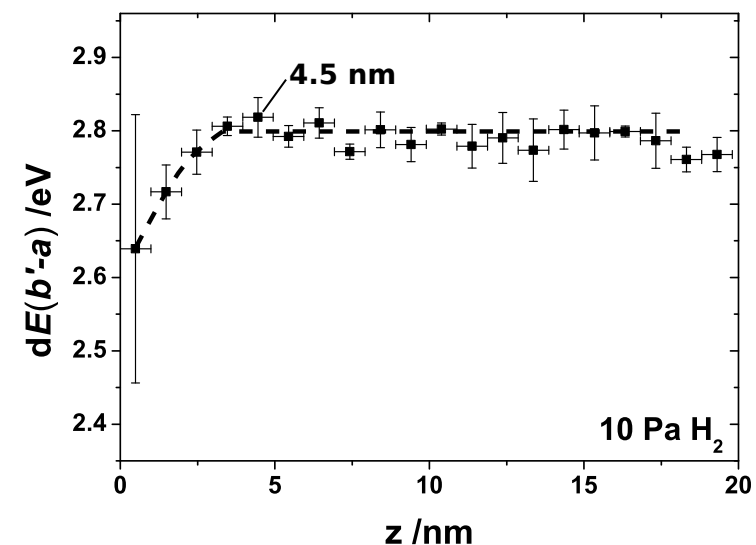

(c)

Figure 4.10.: Energy splitting $\mathrm{d} E\left(b^{\prime}-a\right)$ between the $b^{\prime}$ and $a$ white line as a function of the interface distance $z$. (a) before hydrogen loading and (b) for $1 \mathrm{~Pa}$ hydrogen $\mathrm{d} E\left(b^{\prime}-a\right)$ was constant with $2.8 \mathrm{eV}$ through the $\mathrm{r}-\mathrm{TiO}_{2}$. (c) for $10 \mathrm{~Pa}$ hydrogen $\mathrm{d} E\left(b^{\prime}-a\right)$ decreased in the close vicinity of the interface. The decrease began at a distance of approximately $2 \mathrm{~nm}$ and is $-150 \mathrm{meV}$ at $z=0.5 \mathrm{~nm}$. The dashed lines are shown to highlight the decrease of $\mathrm{d} E\left(b^{\prime}-a\right)$ in the interface vicinity.

values (squares) and uncertainty bars (for different relative position). Since here just bulk $\mathrm{r}-\mathrm{TiO}_{2}$ was measured it would be assumed that all average values are the same.

\footnotetext{
${ }^{2}$ Assuming a one sigma interval.
} 
This oscillating behavior of the data likely originates from instabilities in the microscope. These were small especially in the $10 \mathrm{~Pa}$ case. The stability of the electron loss signal is discussed in more detail in chap. 3.3.1 and 5.3.

The energy splitting $\mathrm{d} E\left(b^{\prime}-a\right)$ at the $\mathrm{Pd} / \mathrm{r}-\mathrm{TiO}_{2}$ interface vicinity was evaluated as a function of the interface distance $z$ for two hydrogen pressures in the ETEM. $\mathrm{d} E\left(b^{\prime}-a\right)$ at $1 \mathrm{~Pa}$ hydrogen is shown against $z$ in $\mathrm{r}-\mathrm{TiO}_{2}$ in fig. $4.10 \mathrm{~b}$.

At $1 \mathrm{~Pa}$ hydrogen, $\mathrm{d} E\left(b^{\prime}-a\right)$ did not reveal any $z$ dependency. $\mathrm{d} E\left(b^{\prime}-a\right)$ was measured as $2.8 \mathrm{eV}$ for all distances $z$ which is the 'bulk' value of $\mathrm{r}-\mathrm{TiO}_{2}$. This situation changed by increasing the hydrogen pressure to $10 \mathrm{~Pa}$, as shown in fig. 4.10c. Here, $\mathrm{d} E\left(b^{\prime}-a\right)$ decreased in close vicinity of the interface. The decrease started approximately $2 \mathrm{~nm}$ from the interface and $\mathrm{d} E\left(b^{\prime}-a\right)$ decreased by approximately $150 \mathrm{meV}$ at a $z$ of approximately $0.5 \mathrm{~nm}$ (cf. fig. 4.10c). Typical for this kind of measurement was the increasing uncertainty in the immediate vicinity of the interface. This feature is discussed later on in chap. 5.5. To prove the observed decrease of $\mathrm{d} E\left(b^{\prime}-a\right)$ in the close vicinity of the interface, SI patterns were taken at different places along the $\mathrm{Pd} / \mathrm{r}-\mathrm{TiO}_{2}$ interface. These measurements are shown in fig. 4.11. All measurements

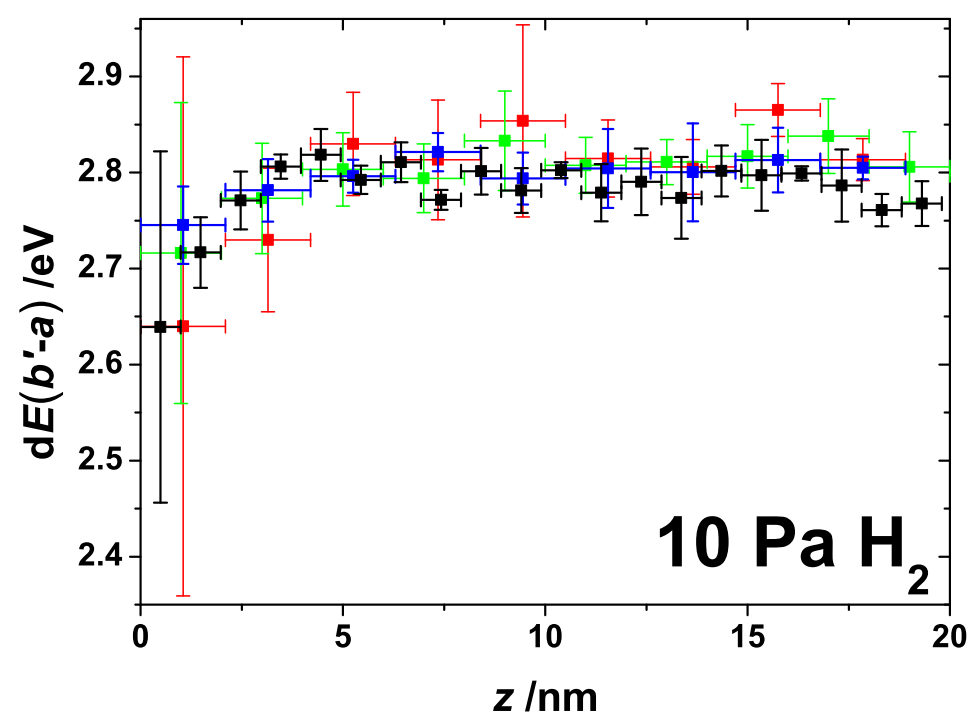

Figure 4.11.: Energy splitting $\mathrm{d} E\left(b^{\prime}-a\right)$ as a function of the interface distance $z$ at $10 \mathrm{~Pa}$ hydrogen. The data from fig. 4.10c are shown in black. The same measurements performed on similar but different places of the $\mathrm{Pd} / \mathrm{r}-\mathrm{TiO}_{2}$ interface are presented with blue, green and red. All spectra revealed qualitatively the same decrease of $\mathrm{d} E\left(b^{\prime}-a\right)$ in the close vicinity of the interface.

revealed the same trend, which confirms the observation that $\mathrm{d} E\left(b^{\prime}-a\right)$ decreases in the close vicinity of the interface.

The decrease of $\mathrm{d} E\left(b^{\prime}-a\right)$ in the close vicinity of the interface can be directly seen in the corresponding EEL spectra. The EEL spectra corresponding to the same $z$ 
value at $0.5 \mathrm{~nm}$ and $1.5 \mathrm{~nm}$ as well as for $4.5 \mathrm{~nm}$ from fig. $4.10 \mathrm{c}$ were averaged. The $4.5 \mathrm{~nm}$ value, which is indicated in fig. $4.10 \mathrm{c}$, represents the far from interface region, since it has the value of the bulk, cf. fig. 4.9. Subsequently, the Ti $L_{3}$ and $L_{2}$ edges were separated at the minimum between them and normalized by the corresponding integral of the intensity $I_{0}$ of the $L_{3}$ and $L_{2}$ edge, respectively. The resulting normed Ti $L_{3}$ and $L_{2}$ edges are separately plotted for different $z$ values in the top graphs of fig. 4.12. The $a$ and $c$ white line and thus the $t_{2 g}$-like states did not reveal any
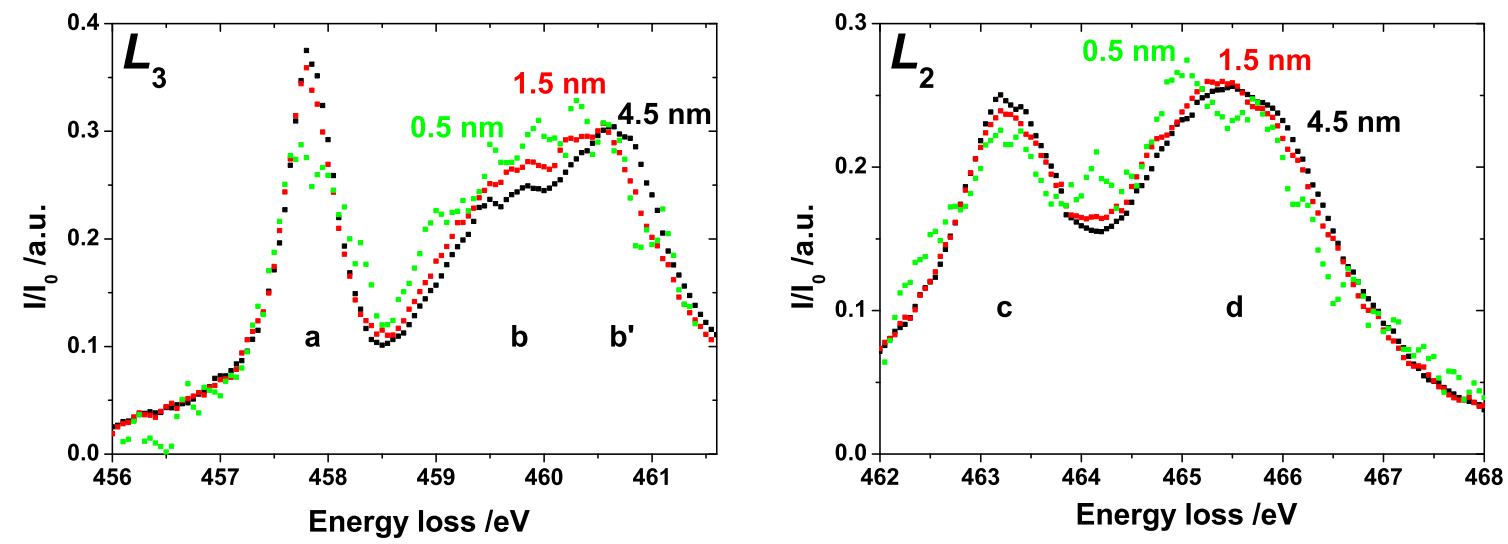

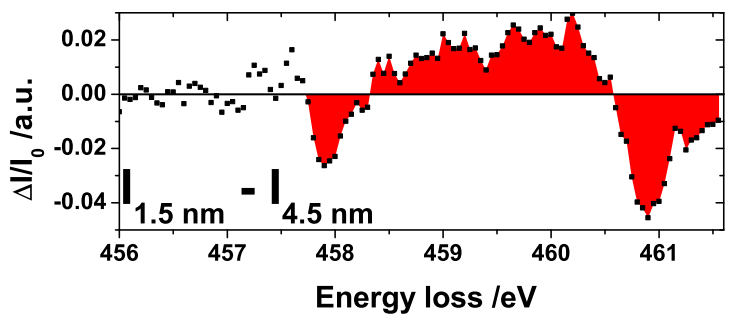

(a)

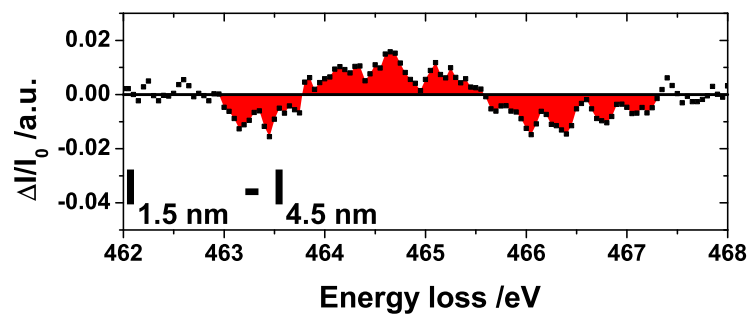

(b)

Figure 4.12.: The Ti (a) $L_{3}$ and (b) $L_{2}$ ELNES is shown at different $z$ in a hydrogen pressure at $10 \mathrm{~Pa}$ in the top graphs. The spectra therefore correspond to the measurements shown in fig. 4.10c. All spectra are normalized to the integrated area of the corresponding edge $I_{0}$. Difference spectra of the normed edges $\Delta I / I_{0}$ between the $1.5 \mathrm{~nm}$ and $4.5 \mathrm{~nm}$ spectrum are shown in the lower part of each graph. The difference is highlighted by the red area. The difference spectra illustrate a change in the $b / b^{\prime}$ and $d$ white line and thus in the $e_{g}$-like states, respectively. This change corresponds to a shift of states in the underlying DoS of $\mathrm{r}-\mathrm{TiO}_{2}$. The average energy resolution for the shown spectra was $350 \mathrm{meV}$.

significant changes except of a small intensity drop for smaller $z$. In contrast, the $b / b^{\prime}$ white line - which correspond to the $e_{g}$-like states - revealed significant changes, cf. fig. 4.12a. The $b^{\prime}$ peak position shifted to lower energy losses for smaller $z$, this finding is already shown in fig. 4.10c. It appeared as if the $b / b^{\prime}$ part shifted to lower energies. A similar but weaker change was also found for the $d$ white line, cf. fig. 4.12b. At this 
point one needs to be careful, the intensity in the $b$ region of the $1.5 \mathrm{~nm}$ spectrum was increased while it was decreased in the $b^{\prime}$ region of the $4.5 \mathrm{~nm}$ spectrum. This suggest a shift of states in the underlying DoS. To support this finding, the difference between the $1.5 \mathrm{~nm}$ and $4.5 \mathrm{~nm}$ spectrum was calculated. The corresponding differences for the $L_{3}$ and $L_{2}$ edge are shown in the bottom graphs of fig. 4.12. The difference spectrum visualizes at what energy losses was intensity removed (negative) or added (positive). In the $L_{3}$ edge, intensity was removed between $457.75 \mathrm{eV}$ and $458.30 \mathrm{eV}$ and between $460.60 \mathrm{eV}$ and $461.55 \mathrm{eV}$, but intensity was added between $458.35 \mathrm{eV}$ and $460.55 \mathrm{eV}$. In the $L_{2}$ edge intensity was removed between $463.00 \mathrm{eV}$ and $463.75 \mathrm{eV}$ and between $465.60 \mathrm{eV}$ and $467.30 \mathrm{eV}$, but added between $463.80 \mathrm{eV}$ and $465.55 \mathrm{eV}$. The changes in the $d$ white line were (in general) less clear since the $L_{2}$ edge experiences a stronger broadening (cf. chap. 2.1.2). The findings in the difference spectra suggest that states were shifted in the DoS which appeared as a shift of approximately $1 \mathrm{eV}$ to $2 \mathrm{eV}$ in the EEL spectra.

In summary, the presence of the $\mathrm{Pd} / \mathrm{r}-\mathrm{TiO}_{2}$ interface resulted in changes to the $\mathrm{Ti}$ $L$ ELNES of $\mathrm{r}-\mathrm{TiO}_{2}$ in the close vicinity $(\lesssim 2 \mathrm{~nm})$ of the interface with a sufficient high hydrogen pressure $(10 \mathrm{~Pa})$. The changes are interpreted as the result of a shift of $e_{g}$-like states in the DoS since mainly the $b / b^{\prime}$ and $d$ white lines were affected. The difference spectra suggest a shift in an energy range of approximately $1 \mathrm{eV}$ to $2 \mathrm{eV}$.

The DoS of $\mathrm{r}-\mathrm{TiO}_{2}$ and the changes in the DoS that are introduced by the presence of defects are discussed in chap. 4.4.

\subsubsection{Hydrogen induced center of gravity (CoG) shifts}

As described in chap. 3.3.3.1, the CoGs of the $a$ and $b / b^{\prime}$ white lines were calculated according to eq. 3.1 for the EEL spectra presented in fig. $4.12 \mathrm{a}$ for the $0.5 \mathrm{~nm}, 1.5 \mathrm{~nm}$ and the $4.5 \mathrm{~nm}$ spectrum. The resulting CoGs were used to calculate their difference $\Delta \mathrm{CoG}$, see eq. 3.2 .

The $\Delta \mathrm{CoG}$ is shown as a function of $z$ in the appendix in fig. A.10. According to eq. 3.3, the relative CoG shift was calculated as a function of $z$ from the $\Delta$ CoG data. For that purpose, the $4.5 \mathrm{~nm}$ EEL spectrum was taken as the reference spectrum since it did not reveal hydrogen related changes. The relative CoG shift is a measure for the distance between the $a$ and $b / b^{\prime}$ white line with respect to a reference. With this quantity, the hydrogen induced changes in the EEL spectra were quantified. This is a complementary approach to the calculation of the energy splitting $\mathrm{d} E\left(b^{\prime}-a\right)$, compare fig. 4.10 and fig. 4.12a.

The relative CoG shift is shown as a function of $z$ in fig. 4.13 . At $z=4.5 \mathrm{~nm}$, the relative CoG shift is 0 since it is the reference. No hydrogen related changes were observed at or above that $z$ distance (cf. fig. $4.10 \mathrm{c}$ ). At $z=1.5 \mathrm{~nm}$, the relative CoG shift was $-(53 \pm 14) \mathrm{meV}$. At $z=0.5 \mathrm{~nm}$ the relative CoG shift was $-(124 \pm 17) \mathrm{meV}$. 
Thus, the CoG of the $e_{g}$ states $\left(\mathrm{CoG}\left(e_{g}\right)\right)$ shifted closer to the $\mathrm{CoG}$ of the $t_{2 g}$ states $\left(\mathrm{CoG}\left(t_{2 g}\right)\right)$.

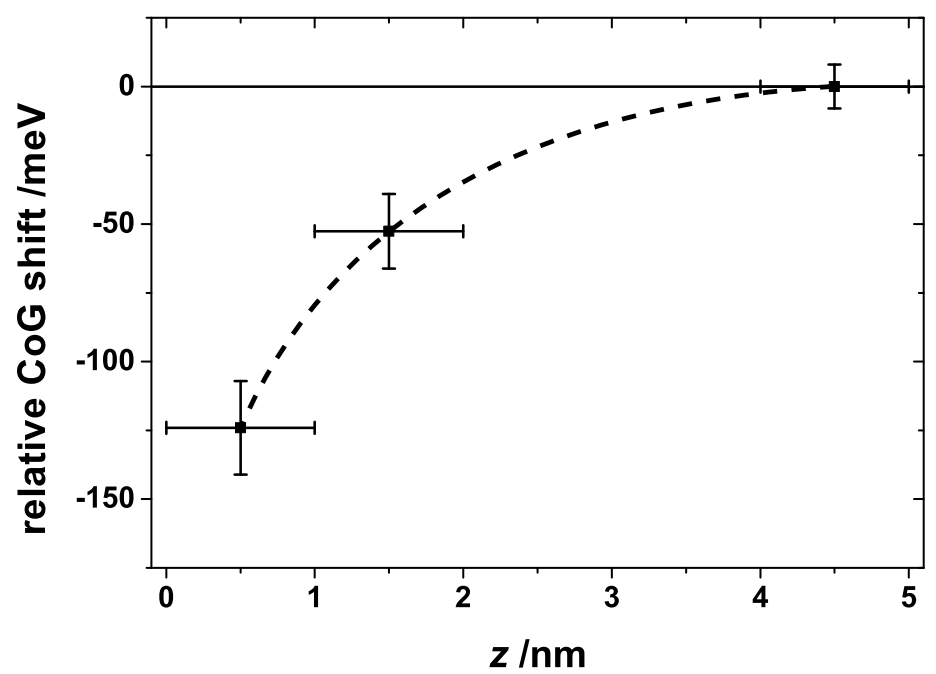

Figure 4.13.: The relative CoG shift, see eq. 3.3, is shown against $z$. The $4.5 \mathrm{~nm}$ spectrum from fig. $4.12 \mathrm{a}$ was taken as the reference. In the close vicinity of the $\mathrm{Pd} / \mathrm{r}-$ $\mathrm{TiO}_{2}$ interface and in a hydrogen pressure of $10 \mathrm{~Pa}$ relative CoG shifts are negative. At $z=1.5 \mathrm{~nm}$ and $z=0.5 \mathrm{~nm}$ the relative CoG shifts were $-(53 \pm 14) \mathrm{meV}$ and $-(124 \pm 17) \mathrm{meV}$, respectively. The dashed line is shown to highlight the trend.

As mentioned in chap. 2.1.2 and 3.3.3.1, the $a$ and $b / b^{\prime}$ white line are closely related to the underlying DoS. In a molecular-orbital picture, the $a$ white line represents the unoccupied $t_{2 g}$ states and the $b / b^{\prime}$ white line represents the unoccupied $e_{g}$ states, cf. fig. 2.6. Therefore, $\triangle \mathrm{CoG}$ and the relative $\mathrm{CoG}$ shift can be compared later on to values for those properties that were calculated by DFT from CoG $\left(t_{2 g}\right)$ and $\operatorname{CoG}\left(e_{g}\right)$.

That such, as the presented, chemical shifts are introduced into the DoS of the $\mathrm{r}-\mathrm{TiO}_{2}$ in the presence of hydrogen and oxygen related defects is shown with DFT calculations in the following section.

\subsection{DFT calculation of oxygen and hydrogen related defects in $\mathrm{r}-\mathrm{TiO}_{2}$}

The initial sample conditions lend to a prediction of $n$-type $\mathrm{r}-\mathrm{TiO}_{2}$ with $\mathrm{V}_{\mathrm{O}}$ as the dominant defect species present after the sample preparation, cf. chap. 5.1. Thus, it is of particular interest how hydrogen is incorporated in $\mathrm{r}-\mathrm{TiO}_{2}$ and how it interacts with $\mathrm{V}_{\mathrm{O}}$. Of particular interest for this work were the changes in the unoccupied part of the DoS since these changes may explain the observed changes in EELS, cf. chap. 4.3. To address this, DFT calculations are presented in this chapter that were 
carried out by M. Sotoudeh and P. Blöchl. The presented DFT calculation have to be considered as preliminary, however they describe the general trends fairly well. More sophisticated calculations will be published in a publication of M. Sotoudeh, M. Bongers, V. Roddatis, J. Čížek, C. Nowak, M. Wenderoth, P. Blöchl and A. Pundt.

Three defects were considered in $\mathrm{r}-\mathrm{TiO}_{2}$ with different charge states: oxygen vacancies $\left(\mathrm{V}_{\mathrm{O}}^{2+,+, 0}\right)$, interstitial hydrogen $\left(\mathrm{H}_{\mathrm{i}}^{+, 0,-}\right)$ and substitutional hydrogen $\left(\mathrm{H}_{\mathrm{O}}^{+, 0,-}\right)$.

\subsubsection{Thermodynamic stability and $\mathrm{Pd} / \mathrm{r}-\mathrm{TiO}_{2}$ interface investigation}

The thermodynamics of $\mathrm{r}-\mathrm{TiO}_{2}$ containing the different defects with their respective charge state (species) is discussed in this section. For that purpose formation energies are analyzed. In addition, a thermodynamic model is applied to investigate the defect concentration in the $\mathrm{r}-\mathrm{TiO}_{2}$ bulk and at an interface to $\mathrm{Pd}$.

Fig. 4.14 shows the thermodynamically stable situation for the different species. Here, the formation energy $\Delta E_{\mathrm{f}}$ of each charged defect is given as a function of the electron chemical potential $\mu_{\mathrm{e}} . \mu_{\mathrm{e}}$ is given relative to the valence band maximum $\epsilon_{\mathrm{vb}}$
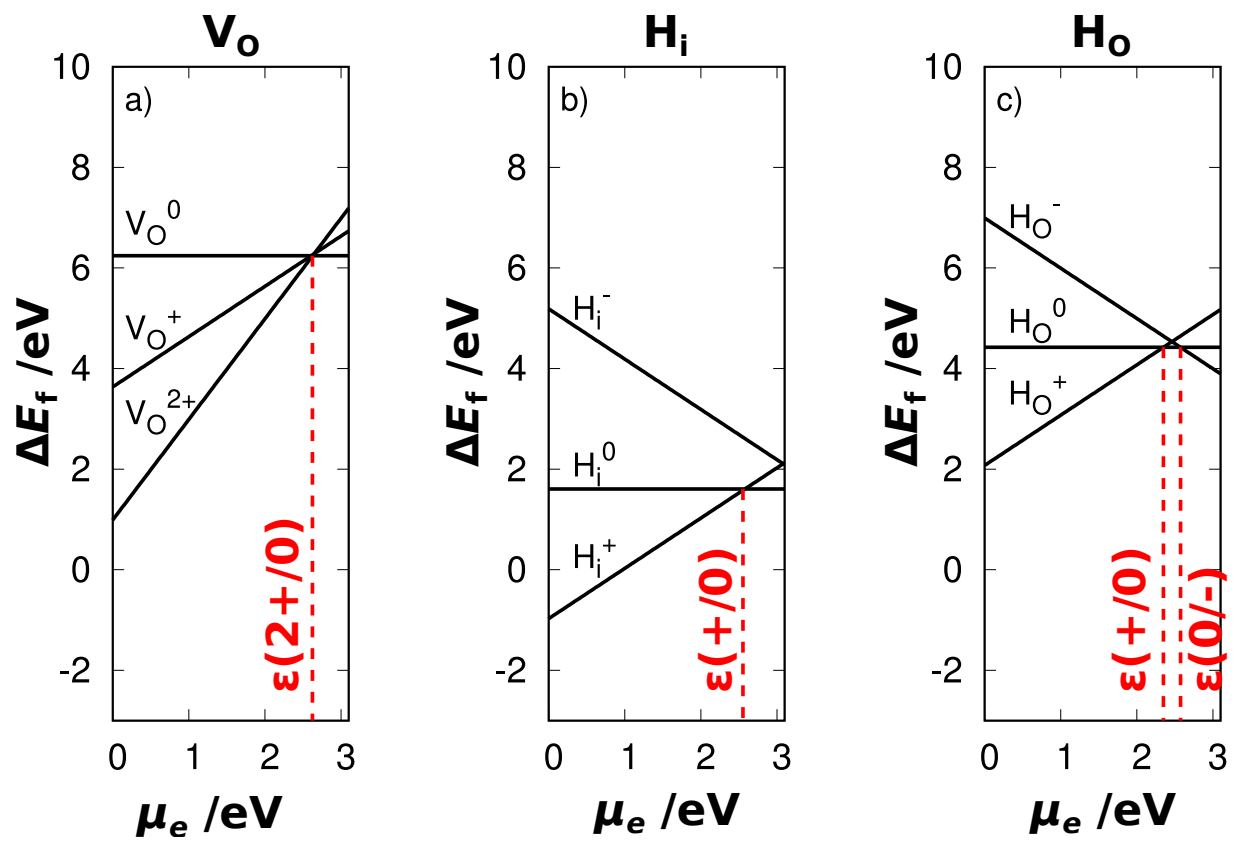

Figure 4.14.: The defect formation energy $\Delta E_{\mathrm{f}}$ is shown against the electron chemical potential $\mu_{\mathrm{e}}$ for $(\mathrm{a})$ the oxygen vacancy $\left(\mathrm{V}_{\mathrm{O}}\right),(\mathrm{b})$ the interstitial hydrogen $\left(\mathrm{H}_{\mathrm{i}}\right)$ and $(\mathrm{c})$ the substitutional hydrogen $\left(\mathrm{H}_{\mathrm{O}}\right) . \mu_{\mathrm{e}}$ is given relative to the valence band maximum $\triangleq \mu_{\mathrm{e}}=0 \mathrm{eV}$. The charge state levels $\epsilon$ are indicated with the red dashed lines. With kind approval of M. Sotoudeh.

and thus ranges from $0 \mathrm{eV}$ to $3.1 \mathrm{eV}$ in the $\mathrm{r}-\mathrm{TiO}_{2}$ band gap. The individual graphs 
show the dependency between $\Delta E_{\mathrm{f}}$ and $\mu_{\mathrm{e}}$ for the $\mathrm{r}-\mathrm{TiO}_{2}$ super cell containing (just) one species.

According to fig. $4.14 \mathrm{a}, \mathrm{V}_{\mathrm{O}}$ is present mainly in the doubly positive $\left(\mathrm{V}_{\mathrm{O}}^{2+}\right)$ and neutral $\left(\mathrm{V}_{\mathrm{O}}^{0}\right)$ charge state over the whole $\mu_{\mathrm{e}}$ range. Close to the charge state level $\epsilon(2+/ 0) \approx 2.63 \mathrm{eV}$ the singly positive charge state $\left(\mathrm{V}_{\mathrm{O}}^{+}\right)$has a $\Delta E_{\mathrm{f}}$ comparable to $\mathrm{V}_{\mathrm{O}}^{2+}$ and $\mathrm{V}_{\mathrm{O}}^{0}$. In this range of $\mu_{\mathrm{e}}, \mathrm{V}_{\mathrm{O}}^{+}$exists in minority, because its formation energy is always higher than of $\mathrm{V}_{\mathrm{O}}^{2+}$ and $\mathrm{V}_{\mathrm{O}}^{0}$. A similar situation was found for $\mathrm{H}_{\mathrm{i}}$, as shown in fig. 4.14b. For the whole range of $\mu_{\mathrm{e}}$, the positive $\left(\mathrm{H}_{\mathrm{i}}^{+}\right)$or neutral $\left(\mathrm{H}_{\mathrm{i}}^{0}\right)$ charge state dominate. The corresponding charge state level is $\epsilon(+/ 0) \approx 2.58 \mathrm{eV}$. The formation energy of the negative charge state $\left(\mathrm{H}_{\mathrm{i}}^{-}\right)$is higher over the whole $\mu_{\mathrm{e}}$ range and hence does not occur in $\mathrm{r}_{-} \mathrm{TiO}_{2}$. For $\mathrm{H}_{\mathrm{O}}$, the situation differs from the other two defects as shown in fig. $4.14 \mathrm{c}$. For $\mu_{\mathrm{e}}$ up to $\epsilon(+/ 0) \approx 2.35 \mathrm{eV}$, the positive charge state $\left(\mathrm{H}_{\mathrm{O}}^{+}\right)$is dominant. At $\epsilon(+/ 0), \mathrm{H}_{\mathrm{O}}^{+}$and the neutral charge state $\left(\mathrm{H}_{\mathrm{O}}^{0}\right)$ coexist. Above $\epsilon(+/ 0)$, $\mathrm{H}_{\mathrm{O}}^{0}$ is dominant up to $\epsilon(0 /-) \approx 2.57 \mathrm{eV}$ at which it coexists with the negative charge state $\left(\mathrm{H}_{\mathrm{O}}^{-}\right)$. Above $\epsilon(0 /-), \mathrm{H}_{\mathrm{O}}^{-}$is the dominating charge state.

The presented thermodynamic stability of the defect raises an interesting question: If more than one defect species is present in $\mathrm{r}-\mathrm{TiO}_{2}$ what defect is most stable? Since $\mathrm{H}_{\mathrm{O}}$ can be seen as the combination of an $\mathrm{V}_{\mathrm{O}}$ and an $\mathrm{H}_{\mathrm{i}}$, it is useful to study the stability of $\mathrm{H}_{\mathrm{O}}$ against the isolated defects $\left(\mathrm{V}_{\mathrm{O}}+\mathrm{H}_{\mathrm{i}}\right)$ as a function of $\mu_{\mathrm{e}}$. This was done by calculating the binding energy $\Delta E_{\mathrm{B}}$ of $\mathrm{H}_{\mathrm{O}}$, which is the difference of the formation energies between the $\mathrm{H}_{\mathrm{O}}$ and the isolated defects $\left(\mathrm{V}_{\mathrm{O}}+\mathrm{H}_{\mathrm{i}}\right)$ :

$$
\Delta E_{\mathrm{B}}\left(\mu_{\mathrm{e}}\right)=\Delta E_{\mathrm{f}}^{\mathrm{H}_{\mathrm{O}}^{q}}\left(\mu_{\mathrm{e}}\right)-\Delta E_{\mathrm{f}}^{\mathrm{V}_{\mathrm{O}}^{q^{\prime}}}\left(\mu_{\mathrm{e}}\right)-\Delta E_{\mathrm{f}}^{\mathrm{H}_{\mathrm{i}}^{q^{\prime \prime}}}\left(\mu_{\mathrm{e}}\right)
$$

Here, $q, q^{\prime}$ and $q^{\prime \prime}$ are the charge states of the respective defects which have the lowest formation energy at the selected $\mu_{\mathrm{e}}$, cf. fig. 4.14.

$\Delta E_{\mathrm{B}}$ is shown as a function of $\mu_{\mathrm{e}}$ in fig. 4.15. Here, the five regimes (a), (b), (c), (d) and (e) can be distinguished. In each regime, the charge states that contribute to $\Delta E_{\mathrm{B}}$ are different and therefore the slope changes, cf. eq. 4.1 and fig. 4.14.

In regime $(\mathrm{a}), \mu_{\mathrm{e}}$ is low and all defects are positively charged. This regime is therefore described by

$$
\mathrm{V}_{\mathrm{O}}^{2+}+\mathrm{H}_{\mathrm{i}}^{+}+2 e^{-} \rightleftharpoons \mathrm{H}_{\mathrm{O}}^{+} \quad \text { for } \mu_{e} \leq 2.35 \mathrm{eV} \text {. }
$$

In regime (a), the binding energy changes its sign from positive to negative at $\mu_{\mathrm{e}} \approx 1 \mathrm{eV}$ as indicated with the red dashed line in fig. 4.15. For $\mu_{\mathrm{e}} \gtrsim 1.0 \mathrm{eV}, \mathrm{H}_{\mathrm{O}}$ is more stable than the isolated defects and is formed preferentially. With increasing $\mu_{\mathrm{e}}, \Delta E_{\mathrm{B}}$ decreases further and $\mathrm{H}_{\mathrm{O}}$ becomes even more stable compared to the isolated defects. The regimes (b) to (e) are described by

$$
\begin{aligned}
\mathrm{V}_{\mathrm{O}}^{2+}+\mathrm{H}_{\mathrm{i}}^{+}+3 e^{-} \rightleftharpoons \mathrm{H}_{\mathrm{O}}^{0} & \text { for } 2.35 \mathrm{eV} \leq \mu_{e} \leq 2.57 \mathrm{eV} \\
\mathrm{V}_{\mathrm{O}}^{2+}+\mathrm{H}_{\mathrm{i}}^{+}+4 e^{-} \rightleftharpoons \mathrm{H}_{\mathrm{O}}^{-} & \text {for } 2.57 \mathrm{eV} \leq \mu_{e} \leq 2.58 \mathrm{eV} \\
\mathrm{V}_{\mathrm{O}}^{2+}+\mathrm{H}_{\mathrm{i}}^{0}+3 e^{-} \rightleftharpoons \mathrm{H}_{\mathrm{O}}^{-} & \text {for } 2.58 \mathrm{eV} \leq \mu_{e} \leq 2.63 \mathrm{eV} \\
\mathrm{V}_{\mathrm{O}}^{0}+\mathrm{H}_{\mathrm{i}}^{0}+e^{-} \rightleftharpoons \mathrm{H}_{\mathrm{O}}^{-} & \text {for } 2.63 \mathrm{eV} \leq \mu_{e}
\end{aligned}
$$




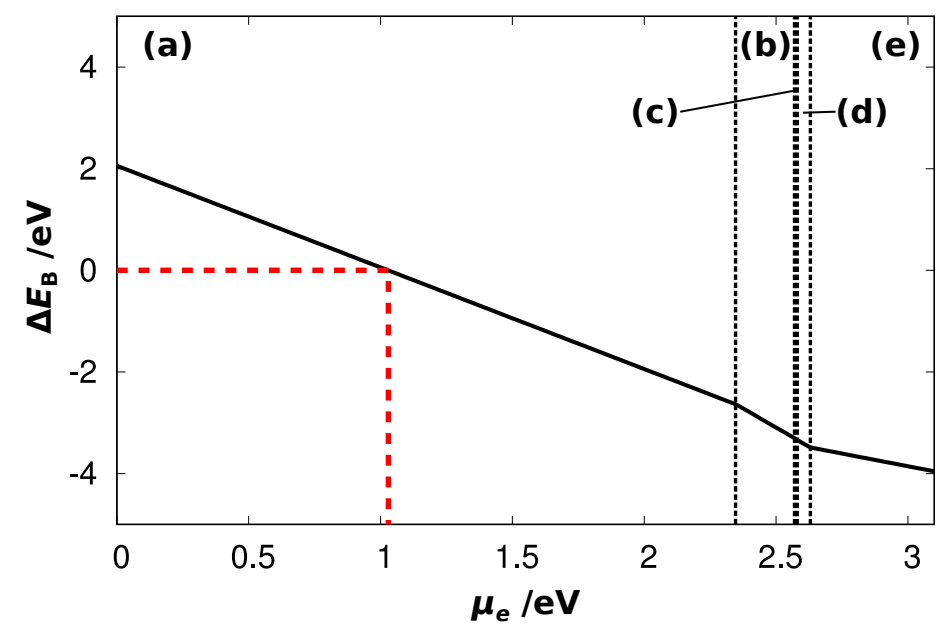

Figure 4.15.: Binding energy $\Delta E_{\mathrm{B}}$ of $\mathrm{H}_{\mathrm{O}}$ against $\mu_{\mathrm{e}} . \Delta E_{\mathrm{B}}$ is the formation energy difference between the $\mathrm{H}_{\mathrm{O}}$ and the isolated defects $\left(\mathrm{V}_{\mathrm{O}}+\mathrm{H}_{\mathrm{i}}\right) \cdot \mu_{\mathrm{e}}$ is given relative to the valence band maximum $\triangleq \mu_{\mathrm{e}}=0 \mathrm{eV}$. Five regimes (a), (b), (c), (d) and (e) are visible. In regime (a) $\Delta E_{\mathrm{B}}$ changes its sign from positive to negative at $\mu_{\mathrm{e}} \approx 1 \mathrm{eV}$ which is indicated by the red dashed line. With kind approval of M. Sotoudeh.

As already mentioned, the slope of $\Delta E_{\mathrm{B}}$ is different in every regime, cf. fig. 4.15. This results from the different slopes of $\Delta E_{\mathrm{f}}$ of the involved defects in every regime, cf. fig. 4.14. However, $\Delta E_{\mathrm{B}}$ is negative above $\mu_{\mathrm{e}} \approx 1 \mathrm{eV}$ resulting in $\mathrm{H}_{\mathrm{O}}$ being more stable and the dominant defect in this $\mu_{\mathrm{e}}$ range.

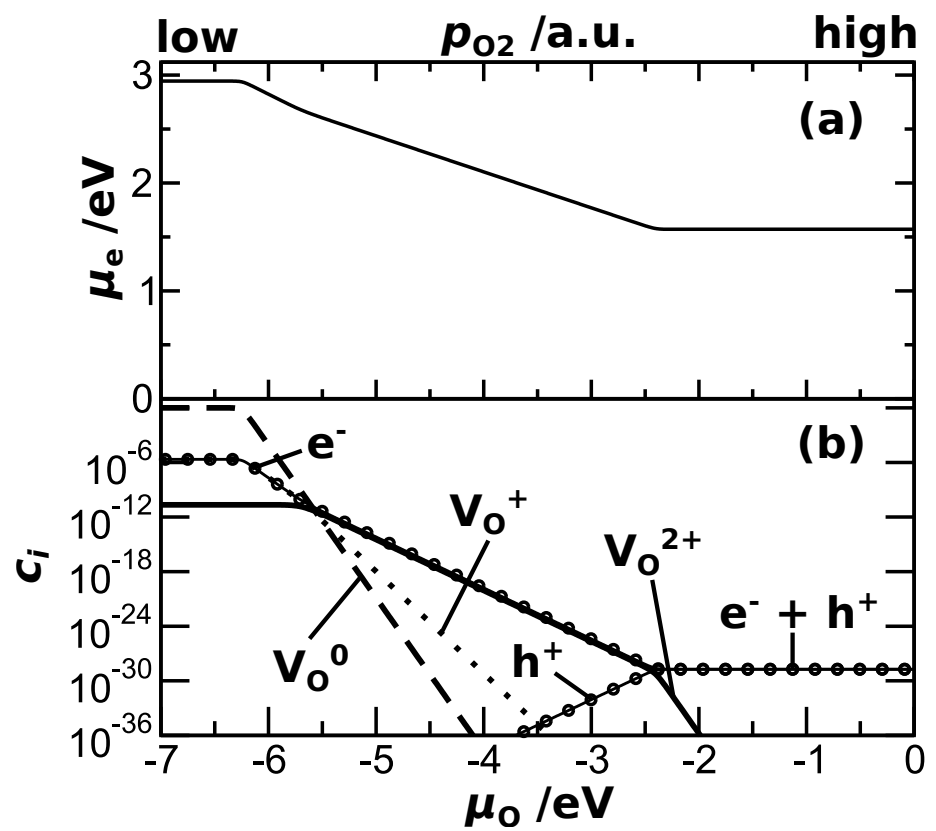

Figure 4.16.: (a) $\mu_{\mathrm{e}}$ and (b) the concentration $c_{i}$ per oxygen site of species $i$ is shown as a function of the oxygen chemical potential $\mu_{\mathrm{O}}$ at $300 \mathrm{~K}$. For high $\mu_{\mathrm{O}}$ (right side) just thermal electron-hole pairs $\left(\mathrm{e}^{-}+\mathrm{h}^{+}\right)$are present. For medium $\mu_{\mathrm{O}}$ values $\mathrm{V}_{\mathrm{O}}^{2+}$ dominate r- $\mathrm{TiO}_{2}$ which are compensated by electrons. For low $\mu_{\mathrm{O}}$ (left side) $\mathrm{V}_{\mathrm{O}}^{0}$ dominates and $\mathrm{r}-\mathrm{TiO}_{2}$ is finally reduced to Ti. With kind approval of P. Blöchl.

A thermodynamic model was applied on $\mathrm{r}-\mathrm{TiO}_{2}$ to investigate the dependency of $\mu_{\mathrm{e}}$ 
and of the concentration $c_{i}$ of the species $i$ on the oxygen chemical potential $\mu_{\mathrm{O}}$. Here, $c_{i}$ is given per oxygen site. No hydrogen is preset in this framework and thus only oxygen vacancies, in any of the three charge states, as well as holes $\mathrm{h}^{+}$and electrons $\mathrm{e}^{-}$were considered as intrinsic species $i$. This investigation was carried out at $300 \mathrm{~K}$ and is shown in fig. 4.16.

According to fig. 4.16, $\mathrm{r}-\mathrm{TiO}_{2}$ shows no influence of $\mathrm{V}_{\mathrm{O}}$ in the approximate interval $[-2.5 \mathrm{eV} ; 0 \mathrm{eV}]$ of $\mu_{\mathrm{O}}$. Here, thermal electron-hole pairs predominate but the pairs are present in very low concentrations $c_{\mathrm{e}^{-}, \mathrm{h}^{+}}$, cf. fig. 4.16b. Therefore, $\mu_{\mathrm{e}}$ is pinned mid-gap at approximately $1.5 \mathrm{eV}$, cf. fig. $4.16 \mathrm{a}$. In the approximate interval $[-5.5 \mathrm{eV}$; $-2.5 \mathrm{eV}]$ of $\mu_{\mathrm{O}}, \mathrm{V}_{\mathrm{O}}^{2+}$ is dominant in $\mathrm{r}-\mathrm{TiO}_{2}$ and compensated by electrons, cf. fig. 4.16b. Its concentration $c_{\mathrm{V}_{\mathrm{O}}^{2+}}$ increases strongly with decreasing $\mu_{\mathrm{O}}$ and the corresponding electrons are supplied to the conduction band. Thus, $c_{\mathrm{e}^{-}}$and $\mu_{\mathrm{e}}$ increase as well. For $\mu_{\mathrm{O}}$ below $-5.5 \mathrm{eV}, \mathrm{V}_{\mathrm{O}}^{0}$ dominates in $\mathrm{r}-\mathrm{TiO}_{2}$, cf. fig. 4.16b. Also, $\mathrm{V}_{\mathrm{O}}^{+}$has a contribution here and supplies further electrons, increasing $\mu_{\mathrm{e}}$ further. But $c_{\mathrm{V}_{\mathrm{O}}^{+}}$stays significantly below $c_{\mathrm{V}_{\mathrm{O}}^{0}}$. At $\mu_{\mathrm{O}}$ of approximately $-6 \mathrm{eV}$ and below, $c_{\mathrm{V}_{\mathrm{O}}^{0}}$ is on the order of unity and $\mathrm{r}-\mathrm{TiO}_{2}$ is no longer stable and reduces to Ti. $\mu_{\mathrm{O}}$ can, in principle, be related quantitatively to the experimental applied oxygen gas pressure $p_{\mathrm{O}_{2}}$. However, for simplicity, a qualitative relationship is drawn on the top in fig. 4.16. Therefore, a high $p_{\mathrm{O}_{2}}$ corresponds to high values of $\mu_{\mathrm{O}}$ and a low $p_{\mathrm{O}_{2}}$ corresponds to low (or more negative) values of $\mu_{\mathrm{O}}$.

Applying the thermodynamic model, a $\mathrm{Pd} / \mathrm{r}-\mathrm{TiO}_{2}$ contact in hydrogen gas environment was simulated by considering purely electrostatic interactions using Poisson's equation. For that purpose, a 'sufficiently' reduced $\mathrm{r}-\mathrm{TiO}_{2}$ was chosen with $\mu_{\mathrm{O}}=-4.35 \mathrm{eV}$, cf. fig. 4.16. The defect concentration $c_{i}$ per oxygen site at the $\mathrm{Pd} / \mathrm{r}-\mathrm{TiO}_{2}$ interface $(z=0)$ and in the bulk of $\mathrm{r}-\mathrm{TiO}_{2}(z \gg 0)$ were investigated as a function of the hydrogen chemical potential ${ }^{3} \mu_{\mathrm{H}}$ as shown in fig. 4.17.

According to fig. 4.17a, in bulk $\mathrm{r}-\mathrm{TiO}_{2}(z \gg 0), \mathrm{H}_{\mathrm{O}}^{0}$ is the dominant defect in the presented $\mu_{\mathrm{H}}$ range. $\mathrm{H}_{\mathrm{O}}^{+}$and $\mathrm{H}_{\mathrm{O}}^{-}$only exist in small quantities, but with equal concentration. At the $\mathrm{Pd} / \mathrm{r}-\mathrm{TiO}_{2}$ interface $(z=0), \mathrm{H}_{\mathrm{O}}^{+}$is the dominant defect, cf. fig. 4.17b. At the calculated interface, nearly no other defects are present. However, an insignificant amount of $\mathrm{H}_{\mathrm{O}}^{0}$ is present. The calculations of the interface suggest that high concentrations up to unity of $\mathrm{H}_{\mathrm{O}}$ related defects can be present depending on $\mu_{\mathrm{H}}$. The experimentally used hydrogen gas pressure of $10 \mathrm{~Pa}$ is indicated, cf. fig. 4.17, and gives a concentration of $\mathrm{H}_{\mathrm{O}}^{+}$on the order of $10 \%$. The different concentrations at the interface and in the bulk $\mathrm{r}-\mathrm{TiO}_{2}$ is obvious. This suggests the presence of a concentration gradient being present from the interface into the bulk $\mathrm{r}-\mathrm{TiO}_{2}$.

The shown results for the thermodynamic model depend in general on $\mu_{\mathrm{O}}$ which was fixed in this case. The calculated concentrations can change as a function of $\mu_{\mathrm{O}}$. A high $\mu_{\mathrm{O}}$, for example, reduces the concentration of hydrogen related defects strongly. However, in that case, the concentrations of the remaining defects are significantly

\footnotetext{
${ }^{3}$ In principle $\mu_{\mathrm{H}}$ can be connected quantitatively to the applied hydrogen gas pressure.
} 
lower than the ones that were shown here.

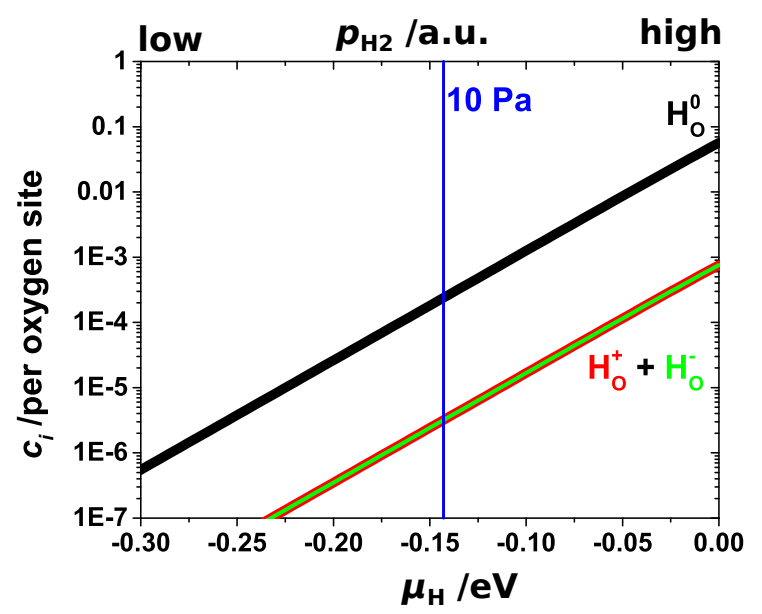

(a) In bulk r- $\mathrm{TiO}_{2}(z \gg 0) \mathrm{H}_{\mathrm{O}}^{0}$ is the dominant defect. $\mathrm{H}_{\mathrm{O}}^{+}$and $\mathrm{H}_{\mathrm{O}}^{-}$exist only in small quantity, but with equal concentration.

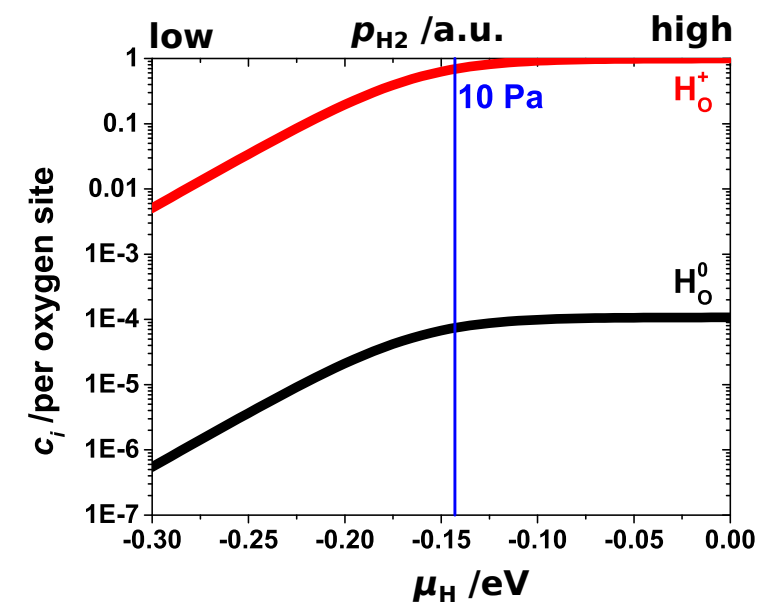

(b) At the $\mathrm{Pd} / \mathrm{r}-\mathrm{TiO}_{2}$ interface $(z=0) \mathrm{H}_{\mathrm{O}}^{+}$is the dominant defect.

Figure 4.17.: Concentration $c_{i}$ of species $i$ given per oxygen site as a function of the hydrogen chemical potential $\mu_{\mathrm{H}}$ in thermodynamic equilibrium. (a) $c_{i}$ in the $\mathrm{r}$ $\mathrm{TiO}_{2}$ bulk and (b) $c_{i}$ at the $\mathrm{Pd} / \mathrm{r}-\mathrm{TiO}_{2}$ interface. The calculations reveal a strong dependency of $c_{i}$ on $\mu_{\mathrm{H}}$. The blue solid line indicates an pressure of $10 \mathrm{~Pa}$, as it was experimentally applied in the in situ EELS studies. All species were considered. However, only $\mathrm{H}_{\mathrm{O}}$ defects were present inconsiderable concentrations. The species concentrations not shown in (a) and (b) were negligible. The oxygen chemical potential was fixed with $\mu_{\mathrm{O}}=-4.35 \mathrm{eV}$. With kind approval of P. Blöchl.

In summary, DFT calculations reveal that the consideration of charged and neutral (hydrogen-related) defects is of significant importance in $\mathrm{r}-\mathrm{TiO}_{2}$, cf. fig. 4.14. These observations are in line with former calculations [50,57]. An investigation of the thermodynamic stability reveals a higher stability of $\mathrm{H}_{\mathrm{O}}$ compared to the individual defects $\mathrm{V}_{\mathrm{O}}$ and $\mathrm{H}_{\mathrm{i}}$ in a broad range of $\mu_{\mathrm{e}}$ in the $\mathrm{r}-\mathrm{TiO}_{2}$ band gap, cf. fig. 4.15. The calculations of the $\mathrm{Pd} / \mathrm{r}-\mathrm{TiO}_{2}$ system in hydrogen gas environment suggest $\mathrm{H}_{\mathrm{O}}$ to be the dominant defect, cf. fig. 4.17. At the interface, the positively charged $\mathrm{H}_{\mathrm{O}}$ dominates and can easily reach concentrations of approximately $10 \%$ per oxygen site. The bulk $\mathrm{r}-\mathrm{TiO}_{2}$ reveals significantly lower defect concentrations with the neutrally charged $\mathrm{H}_{\mathrm{O}}$ as the dominant defect. The considerably different concentrations at the interface and in the bulk suggest the presence of a defect concentration gradient.

Hence, the calculations of the $\mathrm{Pd} / \mathrm{r}-\mathrm{TiO}_{2}$ interface in hydrogen gas environment suggest that $\mathrm{H}_{\mathrm{O}}$ is the most relevant defect in the experimental hydrogen pressure 
range. Therefore, $\mathrm{H}_{\mathrm{O}}$ is investigated in the following sections with respect to its atomic structure, local symmetry and changes that it introduces to the DoS.

\subsubsection{Atomic structure and local symmetry of $\mathrm{H}_{\mathrm{O}}$ in $\mathbf{r}-\mathrm{TiO}_{2}$}

The DFT calculations allow the plotting of the atomic structure of $\mathrm{H}_{\mathrm{O}}$. Its structure is shown in fig. 4.18. In $\mathrm{H}_{\mathrm{O}}$, a hydrogen atom occupies an oxygen site and has therefore

Figure 4.18.: Atomic structure of $\mathrm{H}_{\mathrm{O}}$ in $\mathrm{r}-\mathrm{TiO}_{2}$. The charge state does not influence the qualitative appearance of the structure. Hydrogen occupies an oxygen site and has three Ti atoms as nearest neighbours. The Ti atoms lie within the (110) plane and are arranged in an approximate equilateral triangle which is indicated with the black dashed lines. In fig. 2.3a the same triangle is indicated in the $\mathrm{r}-\mathrm{TiO}_{2}$ unit cell. With kind approval of M. Sotoudeh.

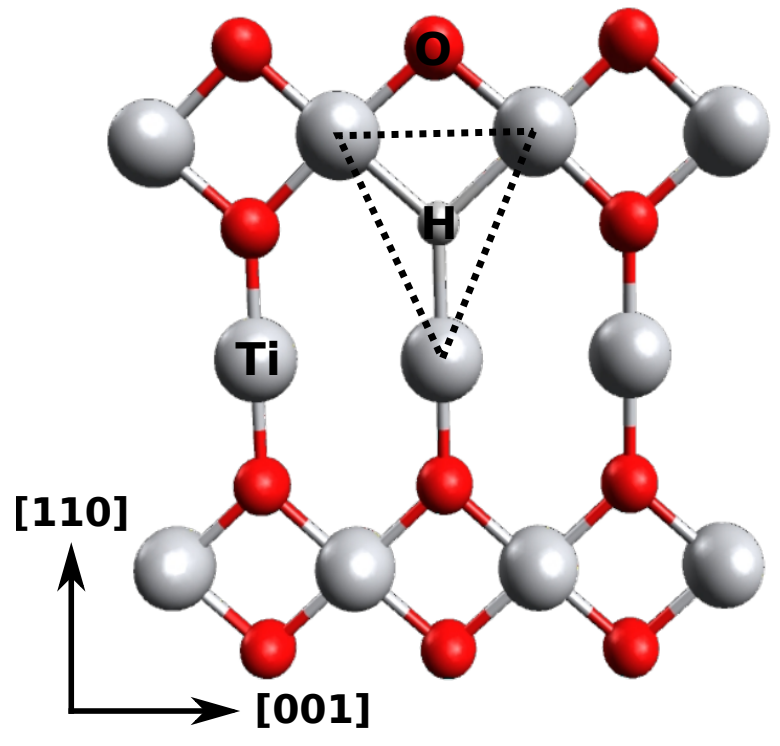

three $\mathrm{Ti}$ atoms as its nearest neighbours. These Ti atoms are lying in the (110) plane and are arranged in an approximate equilateral triangle around the hydrogen atom, cf. black dashed line in fig. 4.18. An equilateral triangle exhibits $\mathrm{D}_{3 \mathrm{~h}}$ symmetry [130, p. 323]. Accompanied by the triangular symmetry, the overlap of the Ti $d$ states exhibit states that correspond to the $A_{1}^{\prime}$ and the $E^{\prime}$ element of the $\mathrm{D}_{3 \mathrm{~h}}$ point group [131]. These are depicted in fig. 4.19. In the $A_{1}^{\prime}$ state, three $\mathrm{Ti} d$ orbitals overlap with the same sign of the wave function (white) in the direction of the defect center, cf. left illustration in fig. 4.19. The two $E^{\prime}$ states are degenerated in energy. One has just two Ti $d$ orbitals with different sign of the wave function (white and gray) that contribute to it, cf. middle illustration in fig. 4.19. The other one has three Ti $d$ orbitals that contribute to it but one $d$ orbital contributes more strongly and its wave function has an opposite sign (cf. right illustration in fig. 4.19). These states appear qualitatively for $\mathrm{H}_{\mathrm{O}}$. Besides the contribution of the $\mathrm{D}_{3 \mathrm{~h}}$ symmetry and its related states, the three Ti atoms still exhibit states that are related to the octahedral arrangement and thus are $t_{2 g^{-}}$and $e_{g^{-}}$like. 


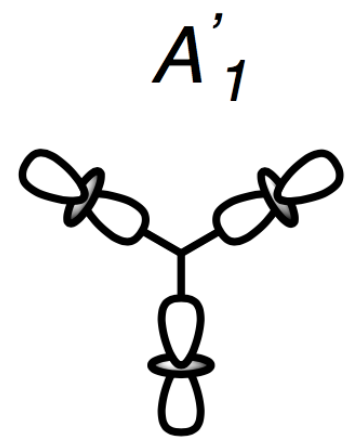

$E^{\prime}$

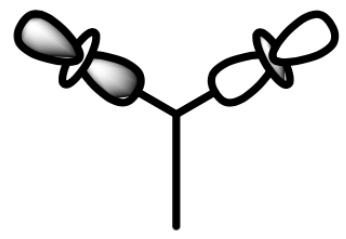

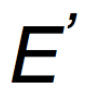

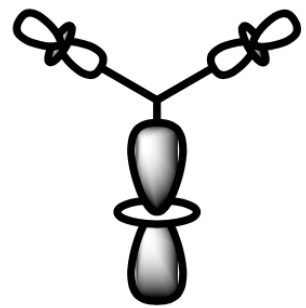

Figure 4.19.: Ti $d$ states that are accompanied by the approximate $\mathrm{D}_{3 \mathrm{~h}}$ symmetry of the three Ti atoms, which are indicated with the dashed line in fig. 4.18. The $d$ orbitals contribute differently to an $A_{1}^{\prime}$ and two degenerated $E^{\prime}$ states. The sign of the wave function is depicted qualitatively with white and gray. With kind approval of M. Sotoudeh.

\subsection{3. $\mathrm{H}_{\mathrm{O}}$ induced changes in the $\mathrm{r}-\mathrm{TiO}_{2}$ density of states (DoS)}

This section presents the $\mathrm{DoS}$ of $\mathrm{r}-\mathrm{TiO}_{2}$ and discusses the changes introduced in the partial DoS of $\mathrm{H}_{\mathrm{O}}$.

The calculated DoS of undoped $\mathrm{r}-\mathrm{TiO}_{2}$ is shown in fig. 4.20. Here, the number of states per energy is shown against the energy $E$ of the calculation. The occupied valence states (up to $0 \mathrm{eV}$ ) are separated by a band gap of $3.1 \mathrm{eV}$ from the unoccupied conduction states (above $3.1 \mathrm{eV}$ ). According to fig. 4.20, the valence states are dominated by oxygen $p$ character whereas the conduction states are dominated by Ti $d$ character. The typical $t_{2 g^{-}} e_{g}$ crystal field splitting is obvious, cf. fig. 4.20. It divides the conduction states in energy in two regions dominated by $t_{2 g^{-}}$and $e_{g}$-like character, respectively, cf. chap. 2.1.1. For energies above the Ti $d$ states $(\gtrsim 10 \mathrm{eV})$, oxygen $p$ states dominate the conduction states. The DoS of the undoped $\mathrm{r}-\mathrm{TiO}_{2}$, as shown in fig. 4.20, undergoes changes in the presence of defects. This is discussed on the example of $\mathrm{H}_{\mathrm{O}}$ in the following.

The changes introduced by the presence of $\mathrm{H}_{\mathrm{O}}$ in the $\mathrm{DoS}$ of $\mathrm{r}-\mathrm{TiO}_{2}$ were investigated. For that purpose, the DoS of the supercell, as well as a partial DoS, were evaluated. The partial DoS was calculated for the Ti $d$ states that correspond to the three Ti atoms neighbouring the defect center, cf. indicated triangle in fig. 4.18. This was carried out for $\mathrm{H}_{\mathrm{O}}^{+}, \mathrm{H}_{\mathrm{O}}^{0}$ and $\mathrm{H}_{\mathrm{O}}^{-}$, which are shown in fig. 4.21, 4.22 and 4.23, respectively.

These figures show the DoS of the supercell including the respectively charged $\mathrm{H}_{\mathrm{O}}$ defect on the top; the partial DoS of $\mathrm{H}_{\mathrm{O}}$ is shown on the bottom. Indicated is the hydrogen contribution in blue and the orbital arrangement at some selected energies in the partial DoS. The DoS of the supercell depends on the concentration of $\mathrm{H}_{\mathrm{O}}$. In contrast to that, the partial DoS depends mainly on the local environment, and 


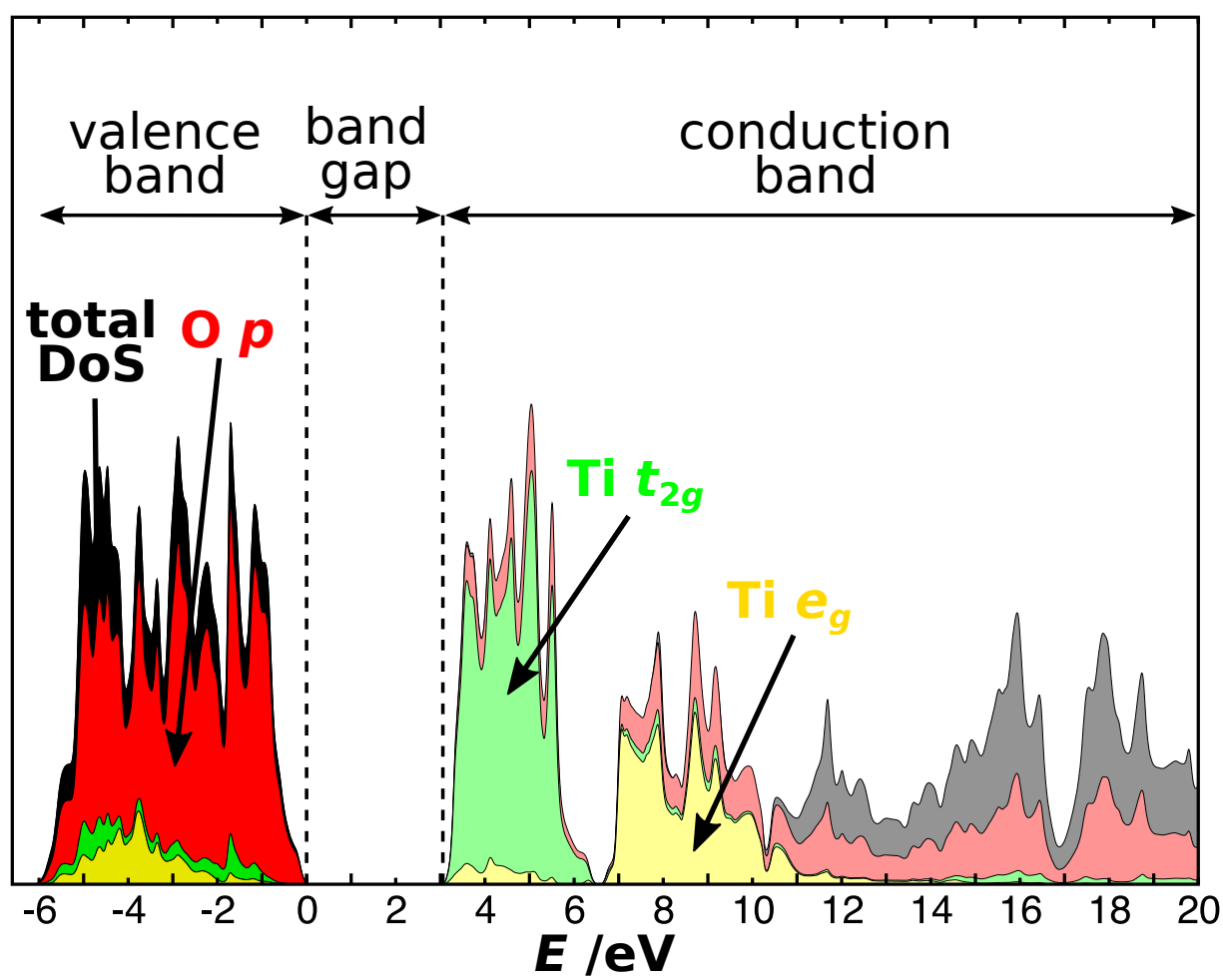

Figure 4.20.: Calculated $\mathrm{DoS}$ of undoped $\mathrm{r}-\mathrm{TiO}_{2}$ in stacked representation. The valence band (or occupied states) is separated from the conduction band (or unoccupied states) via a band gap of $3.1 \mathrm{eV}$. The crystal field splitting in $t_{2 g}$-like states and $e_{g}$-like states are visible, cf. chap. 2.1.1. The indicated colors refer to the total DoS (black) and to DoS that are projected to the following orbitals: oxygen $p$ (red), Ti $t_{2 g}$ (green) and Ti $e_{g}$ (yellow). Unoccupied (or conduction band) states are filled with the corresponding lighter color. With kind approval of M. Sotoudeh.

therefore on the three adjacent $\mathrm{Ti}$ atoms, and is thus nearly independent of the defect concentration. Hence, the partial DoS is expected to change only for extremely high defect concentrations. It is useful to mainly study the partial DoS since the defect induced changes in it are very clear. However, the DoS of the supercell gives a qualitative impression of the defect induced changes which are just valid for the $\mathrm{H}_{\mathrm{O}}$ concentration used for the supercell calculations. The orbital representations show the shape of the respective orbital contribution at selected energies. This allows the identification of the symmetry that dominates the respective orbital arrangement.

According to fig. 4.21, 4.22 and 4.23, the partial DoS graphs reveal a common behavior of $\mathrm{H}_{\mathrm{O}}$ for every charge state: states in the unoccupied part are shifted into the region between the $t_{2 g^{-}}$and $e_{g}$-like states. Furthermore, the contribution of hydrogen to the DoS merges with the valence band and thus has no direct contribution to the shifted Ti $d$ states. According to the orbital arrangement shown in fig. 4.21, in the 


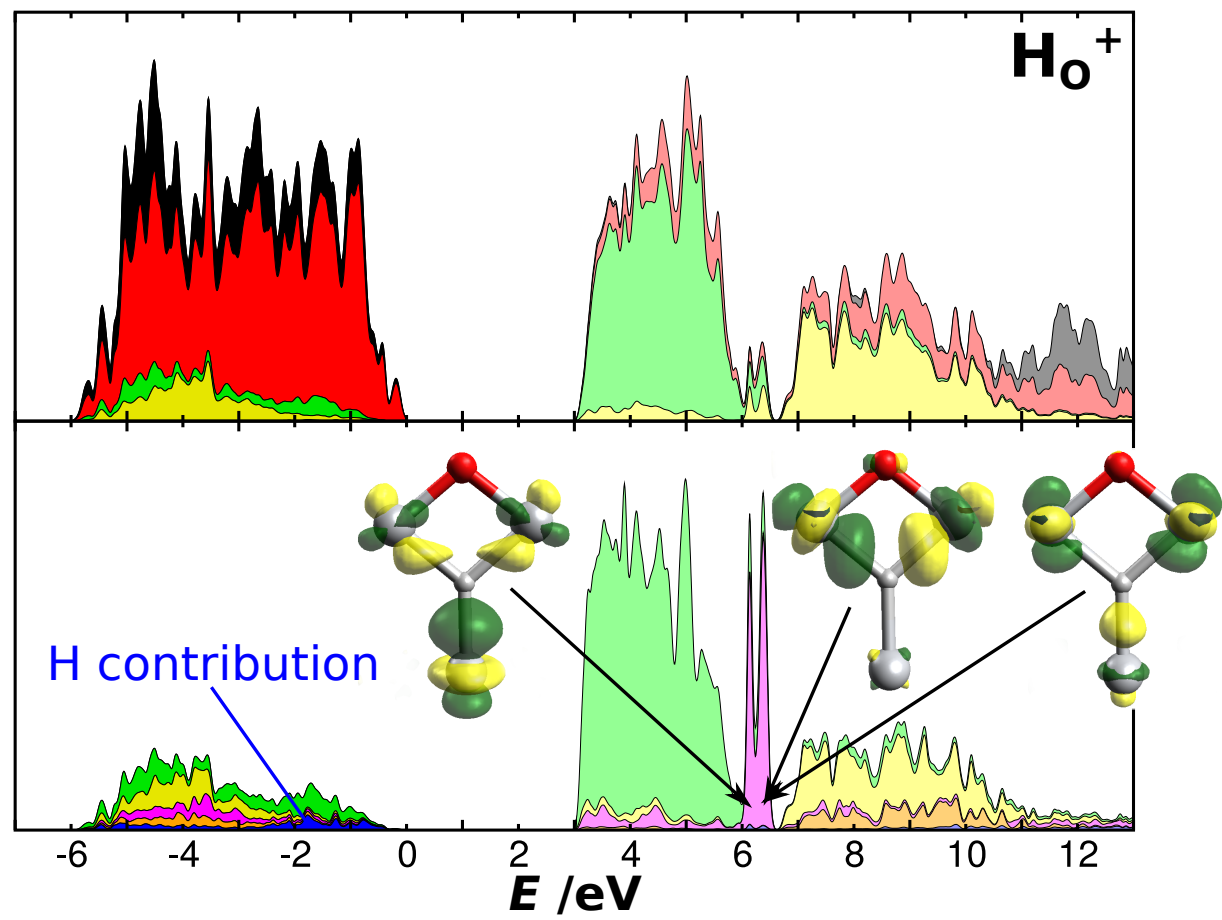

Figure 4.21.: DoS of $\mathrm{H}_{\mathrm{O}}^{+}$in $\mathrm{r}-\mathrm{TiO}_{2}$ in stacked representation. The DoS of the supercell is shown on the top and the partial Ti $d$ DoS on the bottom. The contribution of hydrogen states is shown in blue. The states related to the $\mathrm{D}_{3 \mathrm{~h}}$ symmetry are shown in orange $\left(A_{1}^{\prime}\right)$ and pink $\left(E^{\prime}\right)$, cf. fig. 4.19 . The O $p$ contribution is shown in red, the Ti $t_{2 g}$ contribution in green and the Ti $e_{g}$ contribution in yellow. Unoccupied (or conduction band) states are filled with the corresponding lighter color. Orbital representations at selected energies are added to the figure. States are shifted in between the $t_{2 g^{-}}$and $e_{g}$-like states which reveal $E^{\prime}$ character with respect to the $\mathrm{D}_{3 \mathrm{~h}}$. The respective sign of the wave function is colored with green $(+)$ and yellow (-). With kind approval of M. Sotoudeh.

case of $\mathrm{H}_{\mathrm{O}}^{+}$, the shifted states have mainly $E^{\prime}$ character, cf. fig. 4.19. In the case of $\mathrm{H}_{\mathrm{O}}^{0}$, cf. fig. 4.22 , these states have mainly $E^{\prime}$ character with some $t_{2 g}$ contribution. In the case of $\mathrm{H}_{\mathrm{O}}^{-}$, however, the shifted states are dominated by $t_{2 g}$ character, as can be seen from the four right orbital representations in fig. 4.23. The orbital representations also reveal that the defect states are localized at the three Ti atoms adjacent to the defect center. The defect induced changes in the unoccupied part of the DoS can in principle be measured with EELS.

For $\mathrm{H}_{\mathrm{O}}^{0}$ and $\mathrm{H}_{\mathrm{O}}^{-}$, occupied states appear in the band gap. These states have mainly $t_{2 g}$ character as can be seen from the corresponding orbital representations in fig. 4.22 and 4.23. According to the left orbital representation in fig. 4.22 and 4.23, the corresponding electron and electrons, respectively, are localized on one of the Ti atoms. For 


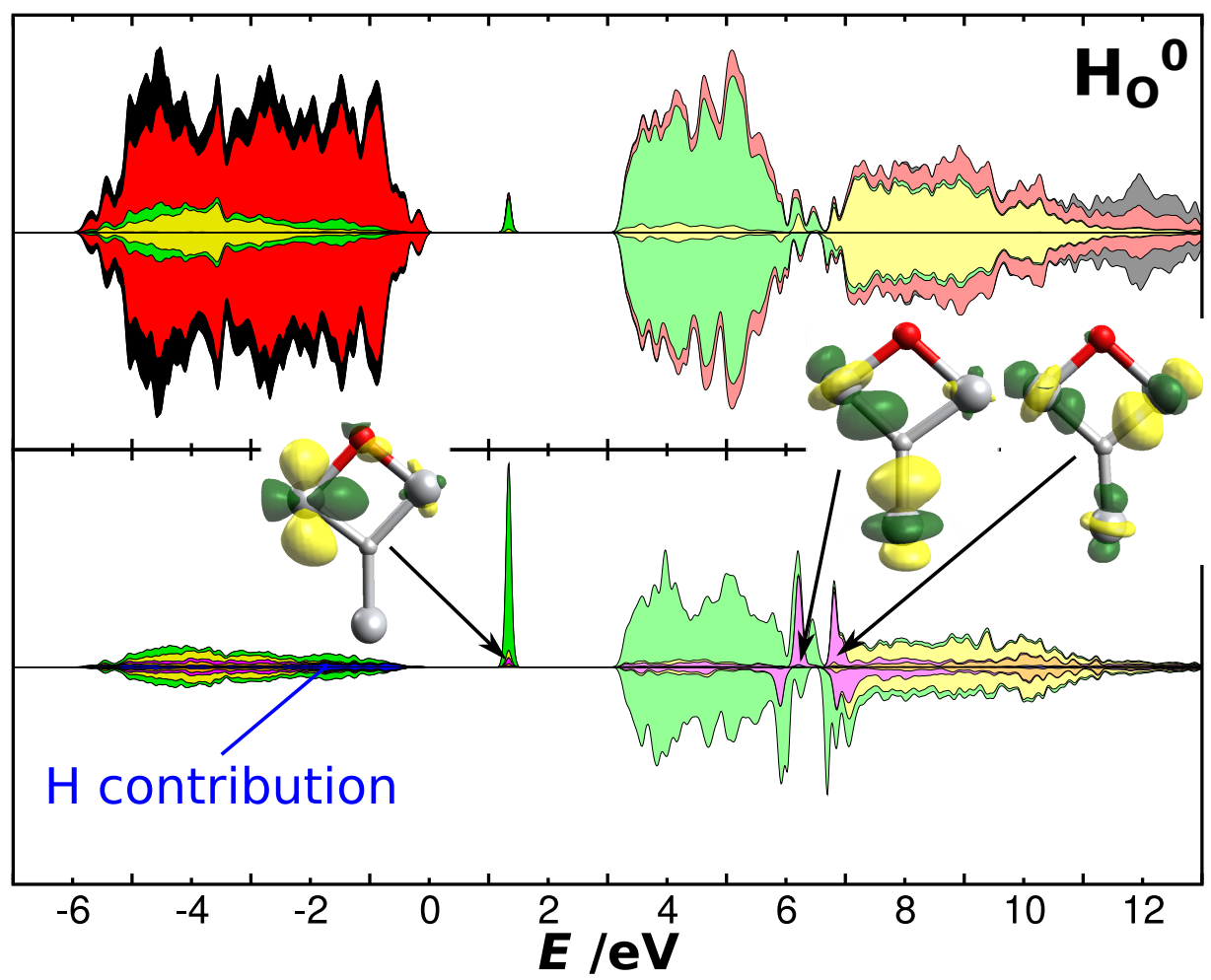

Figure 4.22.: DoS of $\mathrm{H}_{\mathrm{O}}^{0}$ in $\mathrm{r}-\mathrm{TiO}_{2}$ in stacked representation. Legend see fig. 4.21. States are shifted in between the $t_{2 g^{-}}$and $e_{g}$-like states which reveal mainly $E^{\prime}$, with respect to the $\mathrm{D}_{3 \mathrm{~h}}$, and some $t_{2 g}$ character. Additional states with $t_{2 g}$ character appear in the band gap $1.8 \mathrm{eV}$ below the conduction band minimum. With kind approval of M. Sotoudeh.

$\mathrm{H}_{\mathrm{O}}^{0}$ and $\mathrm{H}_{\mathrm{O}}^{-}$, these states lie at $1.8 \mathrm{eV}$ and $1.6 \mathrm{eV}$, respectively, below the conduction band in the band gap. The states in the band gap thus act as deep donors in $\mathrm{r}-\mathrm{TiO}_{2}$. But they are not accessible with EELS since they are occupied.

Important remark: $\mathrm{V}_{\mathrm{O}}$ and $\mathrm{H}_{\mathrm{i}}$ reveal a similar behavior in the $\mathrm{r}-\mathrm{TiO}_{2}$ DoS. Also states shift in the region between $t_{2 g^{-}}$and $e_{g}$-like states. However based on the thermodynamics, they are not expected to contribute significantly to the DoS changes, cf. chap. 4.4.1. Therefore their results are not presented here.

In summary, $\mathrm{H}_{\mathrm{O}}$ (as well as $\mathrm{V}_{\mathrm{O}}$ and $\mathrm{H}_{\mathrm{i}}$ ) in $\mathrm{r}-\mathrm{TiO}_{2}$ introduce shifts in the unoccupied part of the DoS. The shifted states appeared in between the $t_{2 g^{-}}$and $e_{g}$-like states. Such shifts will influence the total DoS of a $\mathrm{r}-\mathrm{TiO}_{2}$ crystal if the defects have sufficient concentrations. Such changes in the unoccupied part of the DoS can, in principle, be measured with EELS as discussed in chap. 2.1.2. Thus, comparing EELS and DoS calculations can give additional insights in the defect assembly of the $\mathrm{Pd} / \mathrm{r}-\mathrm{TiO}_{2}$ system in hydrogen atmosphere. 


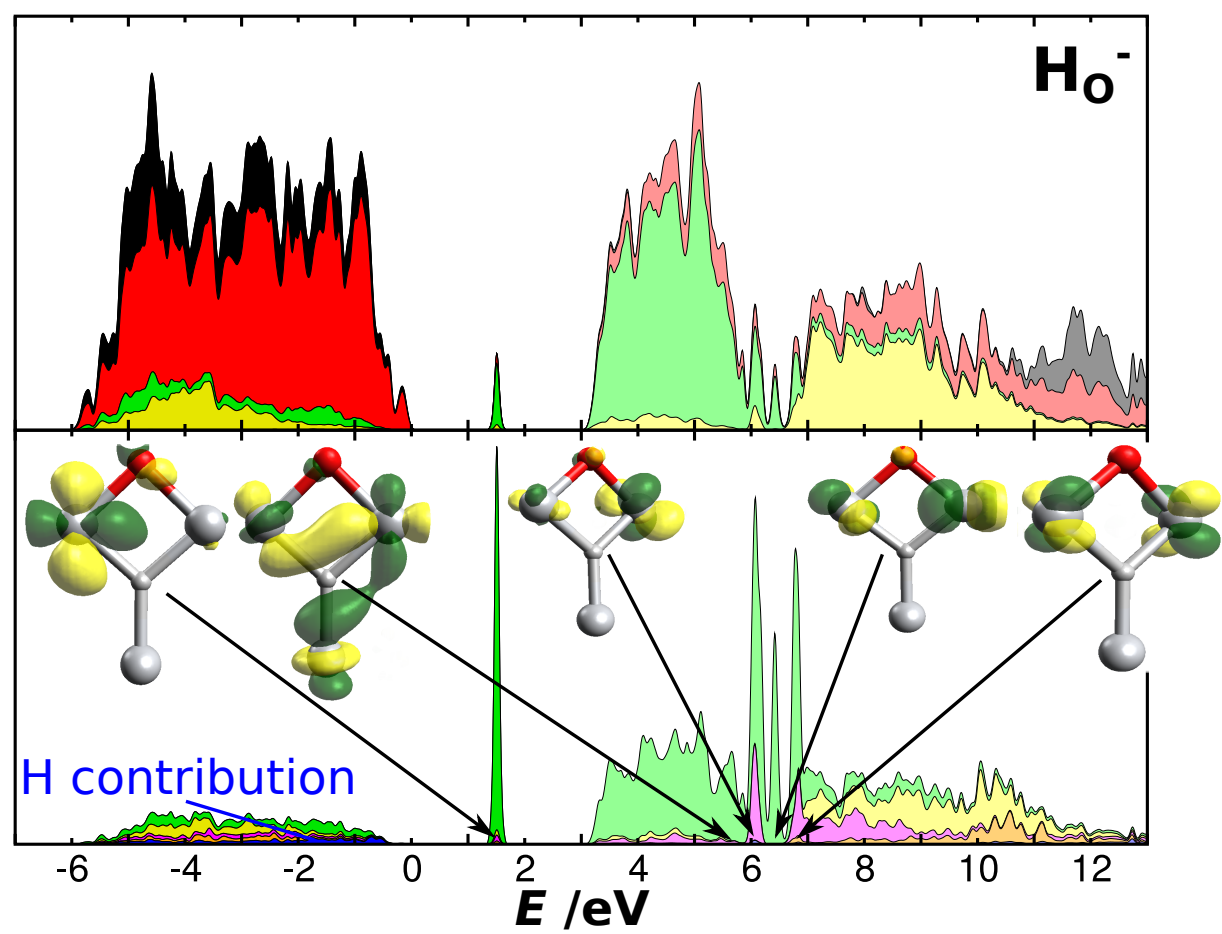

Figure 4.23.: DoS of $\mathrm{H}_{\mathrm{O}}^{-}$in $\mathrm{r}_{-} \mathrm{TiO}_{2}$ in stacked representation. Legend see fig. 4.21. States are shifted in between the $t_{2 g^{-}}$and $e_{g}$-like states which reveal mainly $t_{2 g}$ character. Additional states with $t_{2 g}$ character appear in the band gap $1.6 \mathrm{eV}$ below the conduction band minimum. With kind approval of M. Sotoudeh.

\subsubsection{Center of Gravity (CoG) from the DoS calculations}

The presence of hydrogen induced a shift of states in EEL spectra close to the $\mathrm{Pd} / \mathrm{r}$ $\mathrm{TiO}_{2}$ interface. This shift was quantified by a center of gravity (CoG) evaluation, see chap. 4.3.1. The same evaluation can be applied on the calculated DoS. The procedure of the CoG evaluation is shown in the following.

To be comparable to the CoG evaluation of the EELS measurements, the same approach, see chap. 3.3.3.1, was applied on the Ti $d$ states of the undoped DoS, as shown in fig. 4.24. Only unoccupied conduction states can be measured with EELS. Therefore, the lower boundary for the CoG calculation was chosen as the conduction band minimum at approximately $3.1 \mathrm{eV}$, as indicated with the solid line on the lefthand side in fig. 4.24. The unoccupied Ti $d$ DoS was cut in a $t_{2 g}$ and a $e_{g}$ part by choosing the boundary at the minimum between them which was at approximately $6.3 \mathrm{eV}$, see middle solid line in fig. 4.24. The $e_{g}$ part was cut at an upper boundary which was at approximately $12.8 \mathrm{eV}$, see solid line on the right hand side in fig. 4.24. Above the upper boundary, the DoS contribution is negligible.

The CoG of the $t_{2 g}$ and $e_{g}$ part, namely $\operatorname{CoG}\left(t_{2 g}\right)$ and $\operatorname{CoG}\left(e_{g}\right)$, were calculated 


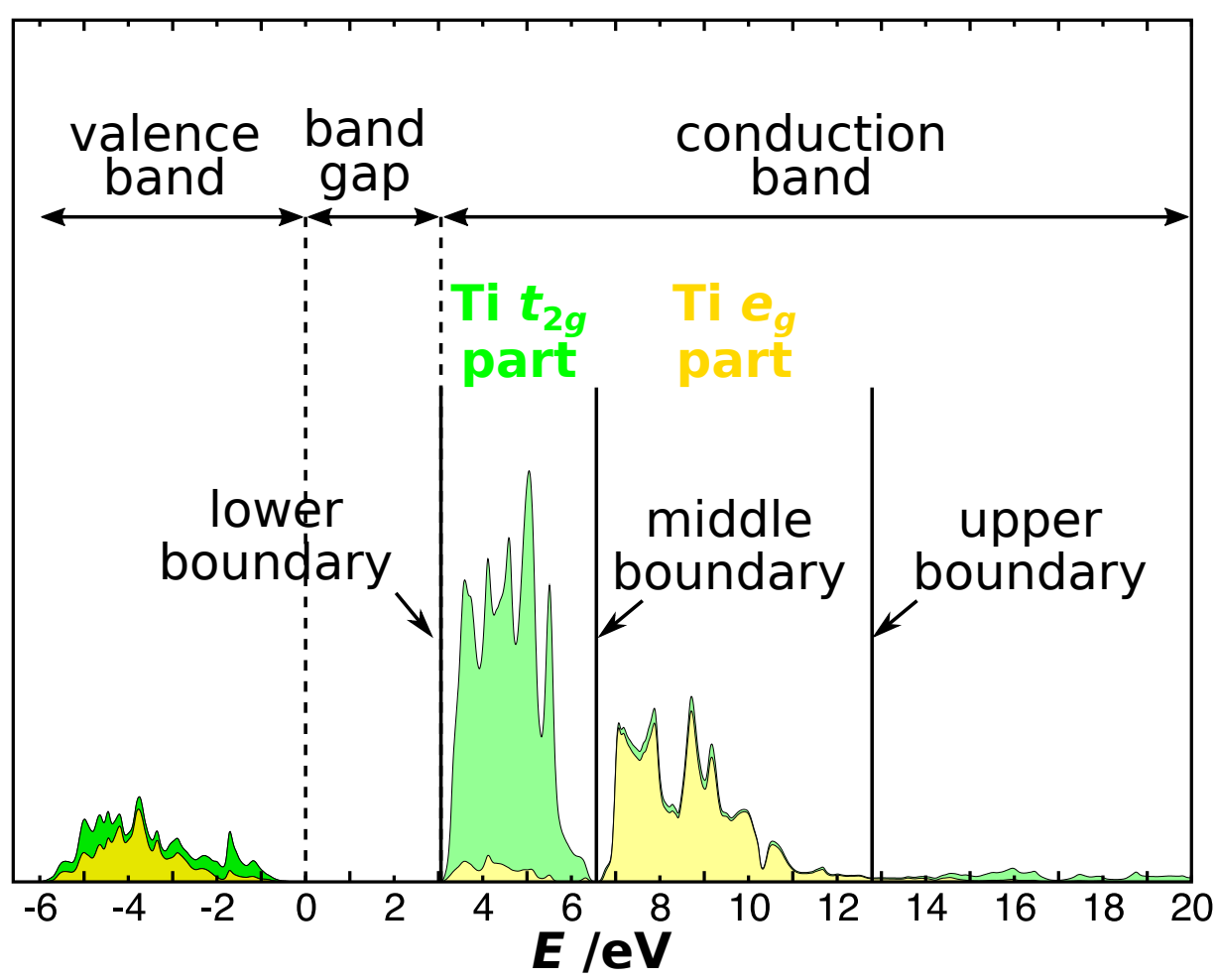

Figure 4.24.: DoS of undoped $\mathrm{r}-\mathrm{TiO}_{2}$, see fig. 4.20, projected on the $\mathrm{Ti} d$ states, namely $t_{2 g}$ (green) and Ti $e_{g}$ (yellow), in stacked representation. The CoG evaluation was applied on this DoS. The corresponding boundaries for the integration are indicated with solid lines. In collaboration with M. Sotoudeh and P. Blöchl.

according to eq. 3.1. For the undoped $\mathrm{r}-\mathrm{TiO}_{2} \mathrm{Ti} d \mathrm{DoS}$, of two Ti atoms, this gives

$$
\begin{aligned}
\operatorname{CoG}\left(e_{g}\right)[\text { undoped }] & =\frac{\int_{e_{g} \text { states }} \text { Ti } d \text { DoS }[\text { undoped }](E) \cdot E \mathrm{~d} E}{\int_{e_{g} \text { states }} \text { Ti } d \text { DoS }[\text { undoped }](E) \mathrm{d} E} \\
& =\frac{58.96373}{3.23879} \mathrm{eV}=18.206 \mathrm{eV} \\
\operatorname{CoG}\left(t_{2 g}\right)[\text { undoped }] & =\frac{\int_{t_{2 g} \text { states }} \text { Ti } d \text { DoS }[\text { undoped }](E) \cdot E \mathrm{~d} E}{\int_{t_{2 g} \text { states }} \text { Ti } d \text { DoS }[\text { undoped }](E) \mathrm{d} E} \\
& =\frac{74.96563}{5.30047} \mathrm{eV}=14.143 \mathrm{eV}
\end{aligned}
$$

The calculated CoG values correspond to the initial energy scale of the calculations and are, therefore, not directly applicable to fig. 4.24. The offset was approximately $8.9 \mathrm{eV}$.

According to eq. $3.2, \Delta \mathrm{CoG}$ is given as

$$
\Delta \mathrm{CoG}[\text { undoped }]=\mathrm{CoG}\left(e_{g}\right)[\text { undoped }]-\mathrm{CoG}\left(t_{2 g}\right)[\text { undoped }] \approx 4.06 \mathrm{eV}
$$


The same evaluation can, in principle, be applied to every $\mathrm{Ti} d$ DoS of $\mathrm{r}-\mathrm{TiO}_{2}$ doped with a certain species (defect + charge state). Thermodynamics predicts $\mathrm{H}_{\mathrm{O}}^{+}$to be the dominant defect at the interface, see fig. 4.17b. Hence, the CoG evaluation was also applied on the Ti $d$ DoS of $\mathrm{r}-\mathrm{TiO}_{2}$ containing $\mathrm{H}_{\mathrm{O}}^{+}$, see partial DoS at the bottom in fig. 4.21. The partial Ti $d$ DoS for $\mathrm{H}_{\mathrm{O}}^{+}$represents the $d$ states from all three Ti atoms neighbouring the defect site, see triangle in fig. 4.18. The same boundary values were used as for the undoped DoS, see fig. 4.24. It is noted, that the partial r- $\mathrm{TiO}_{2} \mathrm{DoS}_{\text {can }}$ exhibit a shift of the whole energy axes with respect to the undoped material. This shift was approximately $0.2 \mathrm{eV}$ in the case of $\mathrm{H}_{\mathrm{O}}^{+}$and is already included in fig. 4.21. The CoGs calculate for the partial Ti $d$ DoS of $\mathrm{H}_{\mathrm{O}}^{+}$as

$$
\begin{aligned}
\operatorname{CoG}\left(e_{g}\right)\left[\mathrm{H}_{\mathrm{O}}^{+}\right] & =\frac{69.01352}{3.7144} \mathrm{eV}=18.580 \mathrm{eV} \\
\operatorname{CoG}\left(t_{2 g}\right)\left[\mathrm{H}_{\mathrm{O}}^{+}\right] & =\frac{127.18798}{8.92842} \mathrm{eV}=14.245 \mathrm{eV}
\end{aligned}
$$

Hence,

$$
\Delta \mathrm{CoG}\left[\mathrm{H}_{\mathrm{O}}^{+}\right]=\operatorname{CoG}\left(e_{g}\right)\left[\mathrm{H}_{\mathrm{O}}^{+}\right]-\mathrm{CoG}\left(t_{2 g}\right)\left[\mathrm{H}_{\mathrm{O}}^{+}\right] \approx 4.33 \mathrm{eV}
$$

With $4.33 \mathrm{eV}$, the $\Delta \mathrm{CoG}$ is larger than for the undoped material with $4.06 \mathrm{eV}$.

A CoG shift can be calculated for $\mathrm{H}_{\mathrm{O}}^{+}$:

$$
\mathrm{CoG} \operatorname{shift}\left[\mathrm{H}_{\mathrm{O}}^{+}\right]=\Delta \mathrm{CoG}\left[\mathrm{H}_{\mathrm{O}}^{+}\right]-\Delta \mathrm{CoG}[\text { undoped }]=0.27 \mathrm{eV}
$$

It is noted, that this CoG shift is not directly comparable to the EELS measurements. However, the sign of the shift shows the direction of the shift. It is obvious that this shift has the opposite sign from the EELS measurements, cf. fig. 4.13. It is very likely that the theoretical calculated CoG shift depends on the choice of the middle boundary for the integration, cf. fig. 4.24. To investigate this issue, the middle boundary was decreased by $-0.5 \mathrm{eV}$. This gives the following values:

$$
\begin{aligned}
\mathrm{CoG}\left(e_{g}\right)[\text { undoped }] & =\frac{59.91917}{3.29922} \mathrm{eV}=18.162 \mathrm{eV} \\
\mathrm{CoG}\left(t_{2 g}\right)[\text { undoped }] & =\frac{74.01018}{5.24005} \mathrm{eV}=14.124 \mathrm{eV} \\
\operatorname{CoG}\left(e_{g}\right)\left[\mathrm{H}_{\mathrm{O}}^{+}\right] & =\frac{86.82079}{4.83103} \mathrm{eV}=17.971 \mathrm{eV} \\
\operatorname{CoG}\left(t_{2 g}\right)\left[\mathrm{H}_{\mathrm{O}}^{+}\right] & =\frac{109.38071}{7.81179} \mathrm{eV}=14.002 \mathrm{eV}
\end{aligned}
$$

and, therefore,

$$
\begin{aligned}
\Delta \mathrm{CoG}[\text { undoped }] & \approx 4.04 \mathrm{eV} \\
\Delta \mathrm{CoG}\left[\mathrm{H}_{\mathrm{O}}^{+}\right] & \approx 3.97 \mathrm{eV}
\end{aligned}
$$


and with it

$$
\text { CoG shift }\left[\mathrm{H}_{\mathrm{O}}^{+}\right]=\Delta \mathrm{CoG}\left[\mathrm{H}_{\mathrm{O}}^{+}\right]-\Delta \mathrm{CoG}[\text { undoped }]=-0.07 \mathrm{eV}
$$

With the decreased value for the middle integration boundary, the calculated shift now has a negative sign. The shown approach to calculate the CoG and the related quantities from the calculated DoS are therefore sensitive to the choice of the integration windows. This is a general limitation for the following comparison of the theoretical to the experimental CoG values.

In summary, the CoGs and their related quantities can be calculated form the partial Ti $d$ DoS. This gives the same approach as for the EEL spectra and can, in principle, allow for a comparison later on. However, the choice of the integration window(s) strongly influence the result.

\subsubsection{A different approach}

The defect induced shift of states in the partial DoS can be quantified. This quantification was performed, by M. Sotoudeh, by integrating the $\operatorname{CoG}\left(t_{2 g}\right)$ and $\operatorname{CoG}\left(e_{g}\right)$ from the conduction band minimum up to high energies $(\sim 20 \mathrm{eV})$. To do so, the Ti $d$ contribution of the three the defect neighbouring $\mathrm{Ti}$ atoms and three $\mathrm{Ti}$ atoms of the undoped $\mathrm{r}-\mathrm{TiO}_{2}$, respectively, were considered. Therefore, no splitting in a $t_{2 g}$ part and $e_{g}$ part of the Ti $d$ states, as done for the previously shown model, was performed. The $\operatorname{CoG}\left(t_{2 g}\right)$ and $\operatorname{CoG}\left(e_{g}\right)$ were calculated for the calculated DoS of the undoped and doped $\mathrm{r}-\mathrm{TiO}_{2}$ as described in chap. 3.8. Subsequently, the difference $\Delta \mathrm{CoG}=\mathrm{CoG}\left(e_{g}\right)-\operatorname{CoG}\left(t_{2 g}\right)$ was calculated for each species.

$\Delta \mathrm{CoG}$ is given for every species in tab. 4.4. The given values are valid for the Ti $d$ states of one defect complex, or, in other words, are given per oxygen site. Hence, they were calculated for the previously discussed partial DoS. The $\Delta$ CoG values correspond to a fully defect filled material in which every oxygen site carries the corresponding defect. As can be seen in tab. 4.4 , every species has smaller $\Delta$ CoG values than the undoped $\mathrm{r}-\mathrm{TiO}_{2}$. Thus, for every defect containing species, the $\mathrm{CoG}\left(e_{g}\right)$ is shifted closer to the CoG $\left(t_{2 g}\right)$. Comparing the differences to the undoped system allowed the calculation of the (theoretical) CoG shift, cf. chap. 3.8. The CoG shift was calculated, as CoG shift[species] $=\Delta$ CoG [species] $-\Delta \mathrm{CoG}$ [undoped], and is given for each species in the third column in tab. 4.4. The defects introduce a negative CoG shift. This shift was found to be between $-126 \mathrm{meV}$ and $-617 \mathrm{meV}$.

Obviously, this approach does not depend on a position of a boundary for the integration, therefore, it is more stable and only shows negative CoG shifts. The discussion about what is the best approach to describe the observed changes in the EELS pattern in the close interface vicinity is still ongoing. However, this approach revealed only negative CoG shifts. 


\begin{tabular}{l|r|r}
\hline \hline species & $\Delta$ CoG $/ \mathrm{eV}$ & CoG shift $/ \mathrm{eV}$ \\
\hline undoped & 3.527 & \\
$\mathrm{H}_{\mathrm{i}}^{+}$ & 3.391 & -0.136 \\
$\mathrm{H}_{\mathrm{i}}^{0}$ & 3.401 & -0.126 \\
$\mathrm{H}_{\mathrm{i}}^{-}$ & 3.041 & -0.486 \\
$\mathrm{~V}_{\mathrm{O}}^{2+}$ & 2.761 & -0.766 \\
$\mathrm{~V}_{\mathrm{O}}^{+}$ & 3.058 & -0.470 \\
$\mathrm{~V}_{\mathrm{O}}^{0}$ & 2.917 & -0.610 \\
$\mathrm{H}_{\mathrm{O}}^{+}$ & 3.239 & -0.289 \\
$\mathrm{H}_{\mathrm{O}}^{0}$ & 3.161 & -0.366 \\
$\mathrm{H}_{\mathrm{O}}^{-}$ & 2.910 & -0.617 \\
\hline \hline
\end{tabular}

Table 4.4.: For every species (defect type + charge state) the difference between the center of gravity $(\mathrm{CoG})$ between the $t_{2 g}$ and $e_{g}$ states $\Delta \mathrm{CoG}=\mathrm{CoG}\left(e_{g}\right)-\operatorname{CoG}\left(t_{2 g}\right)$ is shown. $\triangle \mathrm{CoG}$ of each species is additionally given relative to the undoped $\mathrm{r}-\mathrm{TiO}_{2}$ which gives the $\operatorname{CoG}\left(e_{g}\right)$ shift from the undoped to a defective material. The values of the CoG shifts, thus, correspond to a totally defective material with the corresponding species on each oxygen site. With kind approval of M. Sotoudeh.

In summary, the $\mathrm{CoG}$ shifts introduced by the defects with their corresponding charge state (species) were found to be between $-126 \mathrm{meV}$ and $-617 \mathrm{meV}$, cf. tab. 4.4.

\subsection{Defect concentrations in the $\mathrm{Pd} / \mathrm{r}-\mathrm{TiO}_{2}$ interface vicinity}

A comparison of the theoretically calculated and the experimentally obtained CoGs can, in general, allow the estimation of the defect concentration that yielded to the observed changes in the EEL spectra. However, as mentioned before, the theoretical values are very sensitive to the choice of the integration boundaries, cf. chap. 4.4.4. This limits the estimate of the defect concentration. However, one approach, which was derived by P. Blöchl, to correlate the defect concentration $c$ with the DoS is shown in the following.

The approach is based on the assumption that the changed EEL spectra can be described as a superposition of a defect free material with a defective material. Represented as a DoS, this gives

$$
\operatorname{DoS}[\text { total }](E, c)=\operatorname{DoS}[\text { undoped }](E)+c \cdot\{\text { DoS }[\text { defect }](E)-3 \cdot \operatorname{DoS}[\text { undoped }](E)\}
$$

Here, $c$ is the defect concentration given per oxygen site, DoS[defect] $(E)$ is the partial Ti $d$ DoS of the three Ti atoms neighbouring the defect site, DoS[undoped] $(E)$ is the 
partial $\mathrm{Ti} d$ DoS of one Ti atom in the undoped $\mathrm{r}-\mathrm{TiO}_{2}$ and $\operatorname{DoS}[$ total $](E)$ is the superposition. Clearly, for $c=0, \operatorname{DoS}[$ total $](E, 0)=\operatorname{DoS}[$ undoped $](E)$.

The CoG of DoS[total] is therefore

$$
\operatorname{CoG}(Y)[\operatorname{DoS}[\text { total }](E, c)]=\frac{\int_{Y \text { states }} \operatorname{DoS}[\text { total }](E, c) \cdot E \mathrm{~d} E}{\int_{Y \text { states }} \operatorname{DoS}[\text { total }](E, c) \mathrm{d} E}
$$

Here, $Y$ denotes the states over which the function is integrated.

Based on this equation, the quantity $\mathrm{CoG}$ difference $\Delta \operatorname{CoG}(Y)$ for the states $Y$ can be written as

$$
\Delta \operatorname{CoG}(Y, c)=\operatorname{CoG}(Y)[\operatorname{DoS}[\operatorname{total}](E, c)]-\operatorname{CoG}(Y)[\operatorname{DoS}[\text { total }](E, 0)]
$$

Based on eq. $4.2, \Delta \operatorname{CoG}(Y, c)$ result can be written as:

$$
\Delta \operatorname{CoG}(Y, c)[\text { defect }]=3 c \cdot\{\operatorname{CoG}(Y)[\text { defect }]-\operatorname{CoG}(Y)[\text { undoped }]\} \cdot \frac{A}{1+3 c(A-1)}
$$

Here, $\operatorname{CoG}(Y)[$ defect] is the partial Ti $d$ DoS of the three Ti atoms neighbouring the defect and $\operatorname{CoG}(Y)$ [undoped] is the partial Ti $d$ DoS of one Ti atom in the undoped $\mathrm{r}-\mathrm{TiO}_{2} . A$ is a quantity that is related to both defect and undoped DoS:

$$
A:=\frac{\int_{Y \text { states }} \operatorname{DoS}[\text { defect }](E) \mathrm{d} E}{3 \int_{Y \text { states }} \operatorname{DoS}[\text { undoped }](E) \mathrm{d} E}
$$

In this model, a relative CoG shift can be calculated that should be comparable to the one observed in EELS. The relative CoG shift is defined as, cf. eq. 3.3,

$$
\text { relative CoG shift }[\text { defect }]=\Delta \operatorname{CoG}\left(e_{g}, c\right)[\text { defect }]-\Delta \operatorname{CoG}\left(t_{2 g}, c\right)[\text { defect }]
$$

Based on this model, a comparison can only be drawn if the sign of the shift is equal. As already shown the calculated sign of the shift depends on the choice of the middle boundary for the integration, see chap. 4.4.4. Therefore, to show an applicable example, the values derived for $\mathrm{H}_{\mathrm{O}}^{+}$with the $-0.5 \mathrm{eV}$ shifted middle boundary were chosen.

In EELS, the extension of the $\mathrm{Ti} L_{3}$ edge of $\mathrm{r}-\mathrm{TiO}_{2}$ was measured with approximately $4.25 \mathrm{eV}$, cf. fig. 4.12a. In the calculations, the extension of the DoS was found to be approximately $6.7 \mathrm{eV}$, cf. fig. 4.20. This example shows that an alignment between the calculated and experimental obtained values is needed. The different extensions in experiment and calculation are approximated by the introduction of a proportionality factor $\alpha$. To align $\alpha$, the calculated value of $\Delta \mathrm{CoG}$ [undoped], $4.04 \mathrm{eV}$, is compared to the experimental value of $\Delta \mathrm{CoG}, 2.536 \mathrm{eV}$. The experimentally obtained values of the relative CoG shift, see eq. 3.3 and fig. 4.13, are therefore scaled as

$$
\begin{aligned}
\text { relative CoG shift[EELS comparable to DoS] } & =\alpha \cdot \text { relative CoG shift[EELS] } \\
& =\frac{4.04 \mathrm{eV}}{2.536 \mathrm{eV}} \cdot \text { relative CoG shift[EELS] } \\
& =1.593 \cdot \text { relative CoG shift[EELS] }
\end{aligned}
$$


Thus, the scaled relative CoG shifts from EELS become $-(190 \pm 28) \mathrm{meV}$ at $z=0.5 \mathrm{~nm}$ and $-(80 \pm 22) \mathrm{meV}$ at $z=1.5 \mathrm{~nm}$.

Taking the value of $-190 \mathrm{meV}$ at $z=0.5 \mathrm{~nm}$ for scaled relative CoG shift and applying eq. 4.3 with the values obtained for $\mathrm{H}_{\mathrm{O}}^{+}$with the $-0.5 \mathrm{eV}$ shifted middle boundary, it follows:

$$
\begin{aligned}
-0.190 \mathrm{eV} & =3 c \cdot\{17.971 \mathrm{eV}-18.162 \mathrm{eV}]\} \cdot \frac{0.976}{1+3 c(0.976-1)} \\
& -3 c \cdot\{14.002 \mathrm{eV}-14.124 \mathrm{eV}\} \cdot \frac{0.994}{1+3 c(0.994-1)}
\end{aligned}
$$

Solving this equation for $c$ gives a $\mathrm{H}_{\mathrm{O}}^{+}$concentration of approximately 0.85 per oxygen site. This value is close to one and, hence, already at the limit of this model. Taking the value of $-80 \mathrm{meV}$ at $z=1.5 \mathrm{~nm}$ gives a $\mathrm{H}_{\mathrm{O}}^{+}$concentration of approximately 0.39 per oxygen site.

However, further investigations are needed to improve, for this approach, the comparability between experiment and calculation to obtain suitable values for the concentration.

In summary, the presented model gives an approach to calculate a relative CoG shift that is comparable to the EELS experiments. A rough comparison yields high concentrations being between 0.1 and 1 per oxygen site. But, the obtained results depend strongly on the choice of the middle integration boundary used in the CoG calculations from the DoS.

\subsubsection{A different approach}

The theoretically calculated CoG shifts (see chap. 4.4.4.1) are compared to the CoG shifts observed with EELS (cf. chap. 4.3.1). It is noted, that the EELS evaluation differs from the theoretical evaluation, cf. chap. 4.3.1 and 4.4.4.1. This difference is neglected here for simplicity and the obtained values were used to roughly estimate defect concentrations. To estimate the concentration, it was assumed that the total, with EELS measured, DoS is a superposition of the defect free DoS and the partial DoS of a defect site. Thus, the more defects are present, the stronger is the influence of the partial defects DoS to the total DoS. The second assumption is that one defect is dominant and therefore is the only one contributing significantly to the total DoS. The latter assumption seems reasonable based on the calculation results of the $\mathrm{Pd} / \mathrm{r}-\mathrm{TiO}_{2}$ interface shown in chap. 4.4.1.

The different energy extension of the $t_{2 g}$ and $e_{g}$ states in EELS and the DoS calculations was considered, but differently than in the more sophisticated approach which was presented above. In EELS, the extension of the Ti $L_{3}$ edge of $\mathrm{r}-\mathrm{TiO}_{2}$ was measured with approximately $4.25 \mathrm{eV}$, cf. fig. 4.12a. In the DFT calculations, the extension of the DoS was found to be approximately $6.7 \mathrm{eV}$. Both values give the factor 
that was used for scaling the CoG shifts from EELS (cf. chap. 4.3.1) to the theoretical CoG shifts (cf. tab. 4.4). Thus, the scaled relative CoG shifts from EELS became $-(83 \pm 21) \mathrm{meV}$ at $z=1.5 \mathrm{~nm}$ and $-(196 \pm 27) \mathrm{meV}$ at $z=0.5 \mathrm{~nm}$. This approach is a third assumption because it neglects the non-linearity between EELS and DoS, cf. eq. 2.1 .

The defect concentration per oxygen site (in the $\mathrm{r}-\mathrm{TiO}_{2}(110)$ plane) was estimated using

$$
c_{\text {defect }}=\frac{\text { scaled } \operatorname{CoG}\left(e_{g}\right) \text { shift from EELS }}{\text { theoretical } \operatorname{CoG}\left(e_{g}\right) \text { shift per oxygen site }}
$$

The corresponding results for $c_{\text {defect }}$ are shown in tab. 4.5. Defect concentrations were

\begin{tabular}{l|r|r|r|r}
\hline \hline species & $c_{\text {defect }}(0.5 \mathrm{~nm})$ & uncertainty & $c_{\text {defect }}(1.5 \mathrm{~nm})$ & uncertainty \\
\hline $\mathrm{H}_{\mathrm{i}}^{+}$ & 1.44 & 0.20 & 0.61 & 0.16 \\
$\mathrm{H}_{\mathrm{i}}^{0}$ & 1.55 & 0.21 & 0.66 & 0.17 \\
$\mathrm{H}_{\mathrm{i}}^{-}$ & 0.40 & 0.06 & 0.17 & 0.04 \\
$\mathrm{~V}_{\mathrm{O}}^{2+}$ & 0.26 & 0.03 & 0.11 & 0.03 \\
$\mathrm{~V}_{\mathrm{O}}^{+}$ & 0.42 & 0.06 & 0.18 & 0.05 \\
$\mathrm{~V}_{\mathrm{O}}^{0}$ & 0.32 & 0.04 & 0.14 & 0.04 \\
$\mathrm{H}_{\mathrm{O}}^{+}$ & 0.68 & 0.09 & 0.29 & 0.07 \\
$\mathrm{H}_{\mathrm{O}}^{0}$ & 0.53 & 0.07 & 0.23 & 0.06 \\
$\mathrm{H}_{\mathrm{O}}^{-}$ & 0.32 & 0.04 & 0.23 & 0.03 \\
\hline \hline
\end{tabular}

Table 4.5.: The defect concentration $c_{\text {defect }}$ per oxygen site is given for $z=0.5 \mathrm{~nm}$ and $z=1.5 \mathrm{~nm}$ for the corresponding defect and charge state (species). The presented values were obtained by assuming one dominant defect species that is present in the close vicinity of the $\mathrm{Pd} / \mathrm{r}-\mathrm{TiO}_{2}$ interface and therefore responsible for the $\mathrm{CoG}\left(e_{g}\right)$ shift found with EELS (cf. fig. 4.13). In collaboration with M. Sotoudeh.

calculated that vary between 0.11 and 1.55 per oxygen site. Concentrations above 1 are not described by the calculations and thus cannot explain the observed findings. Thus, $\mathrm{H}_{\mathrm{i}}^{+}$and $\mathrm{H}_{\mathrm{i}}^{0}$ cannot be used to explain the $\operatorname{CoG}\left(e_{g}\right)$ shifts from EELS in the hydrogen environment.

As shown in the calculated thermodynamics, cf. chap. 4.4, $\mathrm{H}_{\mathrm{O}}$ related defects seem very reasonable. At the interface $\mathrm{H}_{\mathrm{O}}^{+}$was predicted to be the dominant defect. According to tab. 4.5, the comparison between experiment and calculations suggest a highly defective material with a $\mathrm{H}_{\mathrm{O}}^{+}$concentration of $(0.68 \pm 0.09)$ per oxygen site.

In summary, a rough estimate of the defect concentration by a comparison of theoretically and experimentally obtained relative $\operatorname{CoG}\left(e_{g}\right)$ shifts revealed that concentrations in the order of $10 \%$ and more are needed to explain the observed EELS findings. Considering $\mathrm{H}_{\mathrm{O}}^{+}$as the dominant defect, as suggested by the thermodynamics, the 
comparison yields a concentration of $(0.68 \pm 0.09)$ per oxygen site in $\mathrm{r}-\mathrm{TiO}_{2}$, in the immediate vicinity of the $\mathrm{Pd} / \mathrm{r}-\mathrm{TiO}_{2}$ interface at $10 \mathrm{~Pa}$ hydrogen gas pressure.

\subsection{Microstructural interface changes in $\mathbf{r}-\mathrm{TiO}_{2}$ due to hydrogen loading}

In addition to the changes in EELS, that suggested a homogeneous change of the close $\mathrm{Pd} / \mathrm{r}-\mathrm{TiO}_{2}$ interface vicinity in the $\mathrm{r}-\mathrm{TiO}_{2}$ in hydrogen atmosphere (cf. chap. 4.3), local changes of the microstructure were observed with HRTEM after hydrogen loading.

Some areas in the vicinity of the interface revealed a changed microstructure, an example is shown in fig. 4.25a. Between the darker $\mathrm{Pd}$ on the top and the grayish

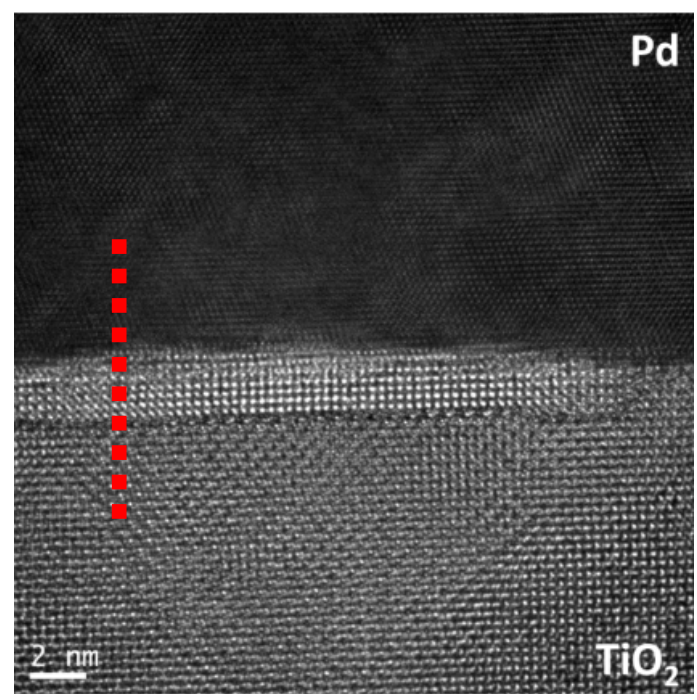

(a)

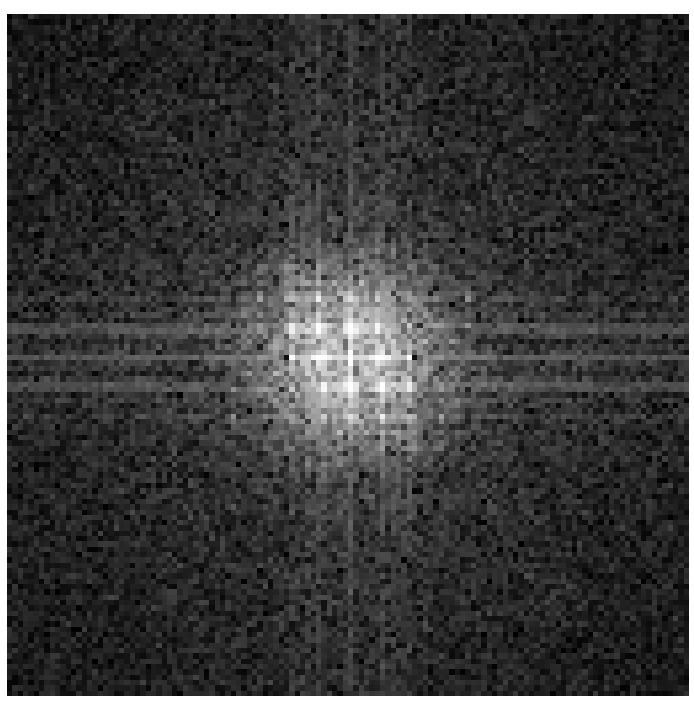

(b)

Figure 4.25.: $\mathrm{Pd} / \mathrm{TiO}_{2}$ interface vicinity after hydrogen was removed from the ETEM. (a) HRTEM revealed that direct at the interface a cavity had formed. These structures formed and grew under hydrogen atmosphere in some parts of the interface region. Their distribution was therefore lateral inhomogeneous. (b) the FFT showed sharp peaks and supported the crystalline structure of the cavity observed in the HRTEM picture. The dashed red line sketches an hypothetical EELS line scan across the cavity region (cf. fig. 4.26a).

$\mathrm{r}-\mathrm{TiO}_{2}$ on the bottom, a brighter area was established at the interface and its vicinity. These, here called cavities, had a spatial extension of approximately $2 \mathrm{~nm}$ orthogonal to the $\mathrm{Pd} / \mathrm{r}-\mathrm{TiO}_{2}$ interface in the $\mathrm{r}-\mathrm{TiO}_{2}$ (/in $z$ direction). Clearly they deviate from the surrounding $\mathrm{r}-\mathrm{TiO}_{2}$. A forward Fourier transform (FFT) of the cavity region is shown in fig. 4.25b. The HRTEM picture as well as the FFT indicates a crystalline 
structure of the cavity but the $\mathrm{S} / \mathrm{N}$ ratio was poor and, unfortunately, a phase analysis on this area was not possible.

A focus series was performed on the cavity region (not shown) indicating a smaller thickness in beam direction. This observation was additionally confirmed using EELS. An EELS line scan over the cavity is shown in fig. 4.26a. In the picture, the height

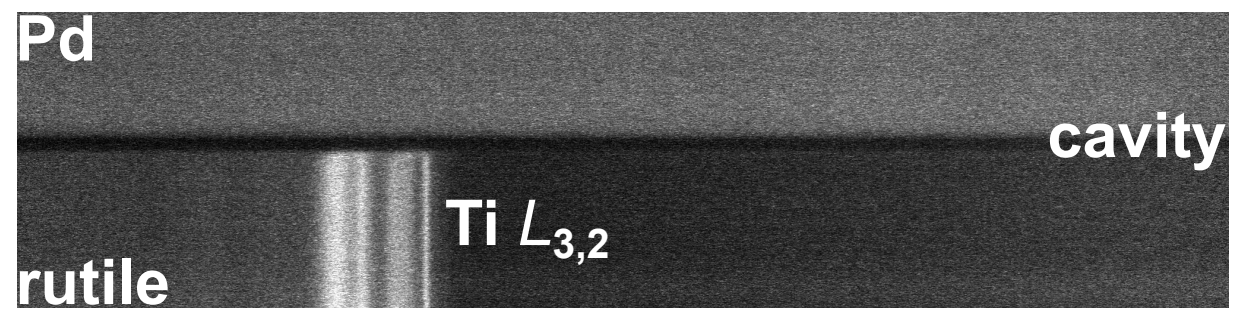

(a)

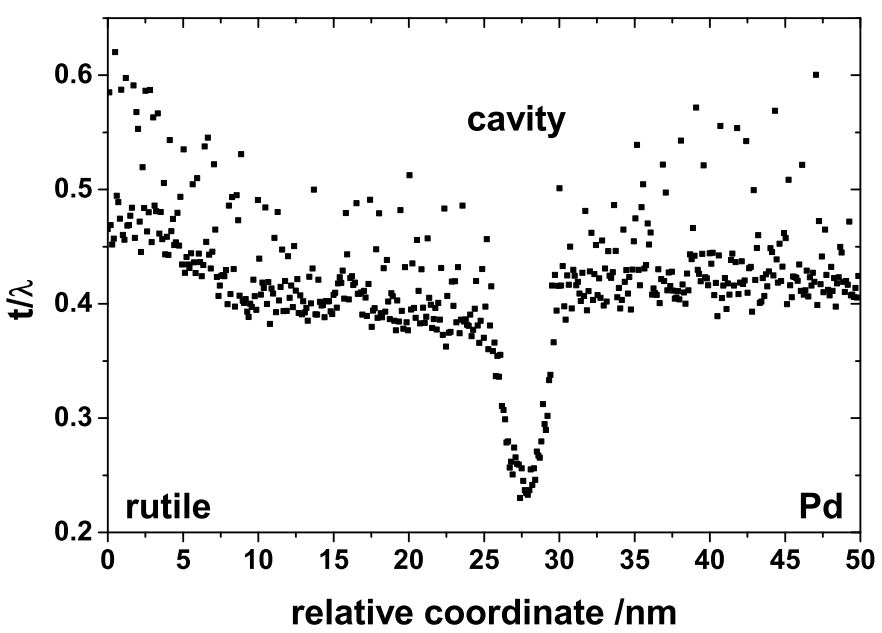

(b)

Figure 4.26.: EELS line scan (cf. dashed red line in fig. 4.25a) of the Ti $L_{3,2}$ edge region across a cavity (a). The height gives the $z$ direction and the width the energy loss. The Ti $L_{3,2}$ edge was not visible in the cavity region with the chosen acquisition conditions. The relative thickness $t / \lambda$ (b) decreased from 0.4 in the surrounding region to 0.16 in the middle of the cavity.

represents a spatial coordinate (as symbolized by the dashed red line in fig. 4.25a) and the width gives the energy loss around the Ti $L_{3,2}$ edge. The Ti $L_{3,2}$ edge was observed in the $\mathrm{r}-\mathrm{TiO}_{2}$ part of the line scan as expected. The acquisition conditions are similar to those for the other EELS experiments (cf. chap. 4.3). In the region of the cavity, no signal from the Ti $L_{3,2}$ edge was observed. In the Pd part, just the plural scattering background was observed. To understand this finding better the relative thickness ${ }^{4}$

${ }^{4} t$ is the thickness of the sample in the direction of the primary electron beam and $\lambda$ is the mean free path of these electrons in the corresponding material of the sample. 
$t / \lambda[39$, chap. 5.1] was evaluated from the plasmon contribution of the EEL low loss signal (not shown). $t / \lambda$ is shown over the $\mathrm{Pd} / \mathrm{r}-\mathrm{TiO}_{2}$ interface region in fig. $4.26 \mathrm{~b}$. Clearly, a thickness drop is visible in the cavity region: $t / \lambda$ decreased from 0.4 in the surrounding to 0.16 in the middle of the cavity. This explained the behavior found in fig. 4.26a: the cavity region is too thin to contribute sufficient signal with the chosen acquisition parameters and it confirmed the results from the focus series.

In summary, observation of the cavity region shows that it is significantly thinner than the surrounding $\mathrm{r}-\mathrm{TiO}_{2}$. This suggest that mass is lost in a certain (reaction) channel. Important to note is that there was no contribution of the cavities to the measured Ti $L_{3,2}$ ELNES with the chosen EELS acquisition parameters.

\subsection{PAS measurements on hydrogen loaded $\mathrm{Pd} / \mathrm{r}-\mathrm{TiO}_{2}$}

VEPAS measurements were performed on a $\sim 200 \mathrm{~nm} \mathrm{Pd} / \mathrm{r}-\mathrm{TiO}_{2}$ sample, in collaboration with Jakub Č́žzek, from Charles University Prague. The $S$ parameter was evaluated as a function of the incident energy of the positrons, and thus as a function of the positron's penetration depth, and of the hydrogen pressure (cf. chap. 3.4). The corresponding VEPAS measurements are depicted in fig. 4.27. At $E<1 \mathrm{keV}$,

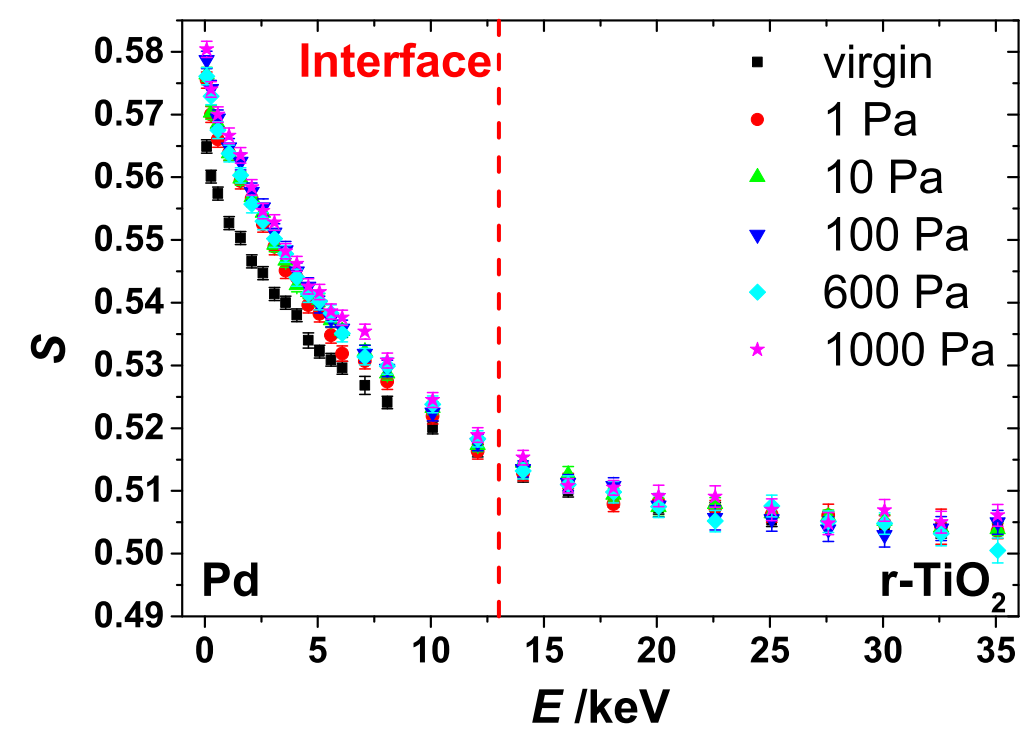

Figure 4.27.: VEPAS on a $200 \mathrm{~nm} \mathrm{Pd}$ film deposited at $1023 \mathrm{~K}$ on $\mathrm{r}-\mathrm{TiO}_{2}$. The $S$ parameter is shown as a function of the incident energy of the positrons $E$, and thus as a function of the penetration depth, and of the hydrogen pressure. Adapted with permission from [132]. Copyright 2018 American Chemical Society.

the positrons annihilated mainly at the Pd surface. With increasing $E$, the positrons 
penetrated further into the $\mathrm{Pd}$ film, therefore the amount of positrons diffusing back to the surface decreased and, with this, the $S$ parameter. At approximately $13 \mathrm{keV}$, the positrons had a mean penetration depth of $200 \mathrm{~nm}$, which corresponded to the $\mathrm{Pd}$ film thickness. Thus, for $E>13 \mathrm{keV}$, the positrons penetrated into the r- $\mathrm{TiO}_{2}$. For $E>30 \mathrm{keV}, S$ reached the bulk value since nearly all positrons were annihilated in the $\mathrm{r}-\mathrm{TiO}_{2}$. This general trend was found for all tested hydrogen pressures, cf. fig. 4.27. With increasing hydrogen pressure, no changes were found in the bulk or the (close) interface vicinity of the $\mathrm{r}-\mathrm{TiO}_{2}$ even up to $1000 \mathrm{~Pa}$ hydrogen. This indicated that the concentration of titanium vacancies, cf. chap. 3.4, did not change or was below the detectable limit.

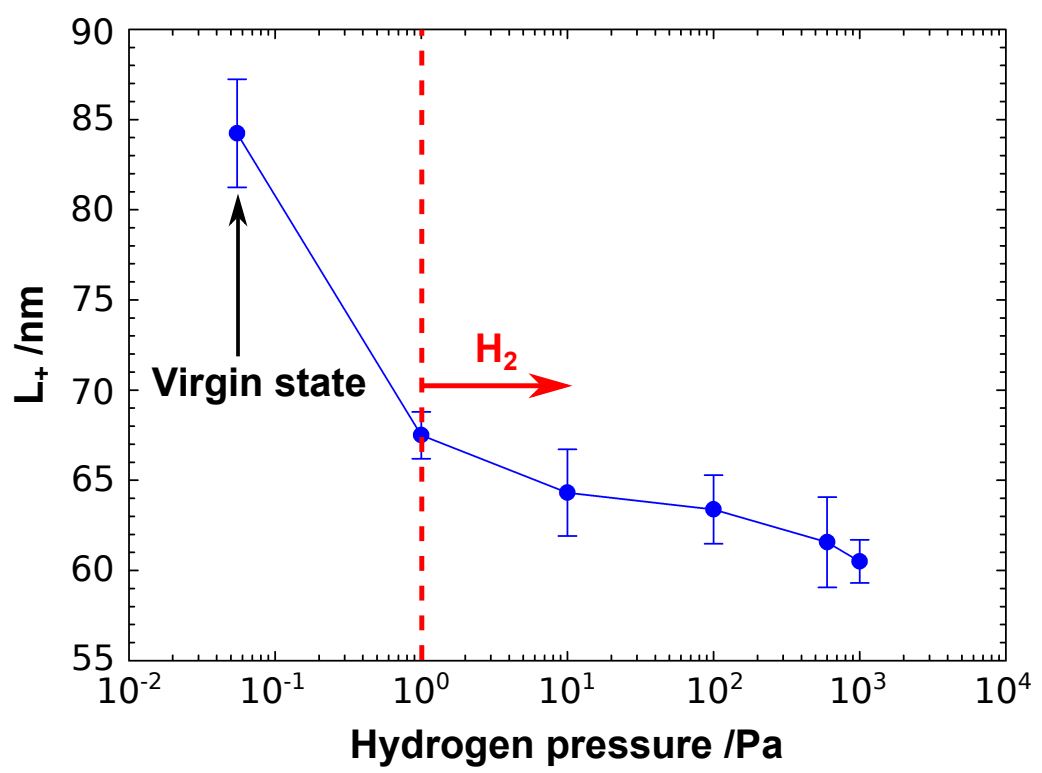

Figure 4.28.: The average diffusion length $\left(\mathrm{L}_{+}\right)$of the positrons in the $\mathrm{Pd}$ film is shown as a function of the applied hydrogen gas pressure. $\mathrm{L}_{+}$was calculated from $S(E)$, cf. fig. 4.27. In the virgin state, $\mathrm{L}_{+}$is approximately $85 \mathrm{~nm}$. $\mathrm{L}_{+}$decreases to $67 \mathrm{~nm}$ at $1 \mathrm{~Pa}$ hydrogen and even further to approximately $60 \mathrm{~nm}$ at $1000 \mathrm{~Pa}$. Therefore, hydrogen loading introduced additional defects that create open volume for positron trapping. In collaboration with Jakub Čížek.

Changes, however, of the $S$ parameter were found in the $\mathrm{Pd}$ film $(E<13 \mathrm{keV})$ : the $S(E)$ parameter increased with increasing hydrogen pressure. In general, this indicates an increased concentrations of open volume defects in the $\mathrm{Pd}$ film under hydrogen loading, cf. chap. 3.4. Open volume defects in the high crystalline Pd film can be vacancies $\mathrm{V}_{\mathrm{Pd}}$ and dislocations $\perp_{\mathrm{Pd}}$. The additionally created open volume that was introduced yields to a decreasing average diffusion length $\mathrm{L}_{+}$of the positrons in the Pd film. $\mathrm{L}_{+}$was evaluated by Jakub Čížek by a fit of the $S(E)$ curves. The 
average diffusion length of the positrons in the $\mathrm{Pd}$ film is shown as a function of the applied hydrogen gas pressure in fig. 4.28. Here, it can be seen that the diffusion length of the positrons is around $85 \mathrm{~nm}$ in the virgin state before hydrogen loading. Introducing $1 \mathrm{~Pa}$ hydrogen yielded a significant decrease of $\mathrm{L}_{+}$to approximately $67 \mathrm{~nm}$. Increasing the hydrogen pressure further was accompanied by a further decrease of $\mathrm{L}_{+}$ to approximately $60 \mathrm{~nm}$ at $1000 \mathrm{~Pa}$ hydrogen, cf. fig. 4.28. Based on the $\mathrm{L}_{+}$data obtained from the VEPAS measurements, it was possible to calculate the change in the average vacancy concentration $\Delta c_{\mathrm{V}}$ in the $\mathrm{Pd}$ film by a fit of the $S(E)$ parameter. To calculate the concentration change, the virgin state of the sample was used as the reference. The calculation of $\Delta c_{\mathrm{V}}$ was carried out by Jakub Ćížek under the assumption that vacancies were the dominant open volume defects. Based on this assumptions, the change in the average vacancy concentration $\Delta c_{\mathrm{V}}$ in the $\mathrm{Pd}$ film is shown as a function of the applied hydrogen gas pressure in fig. 4.29. The virgin

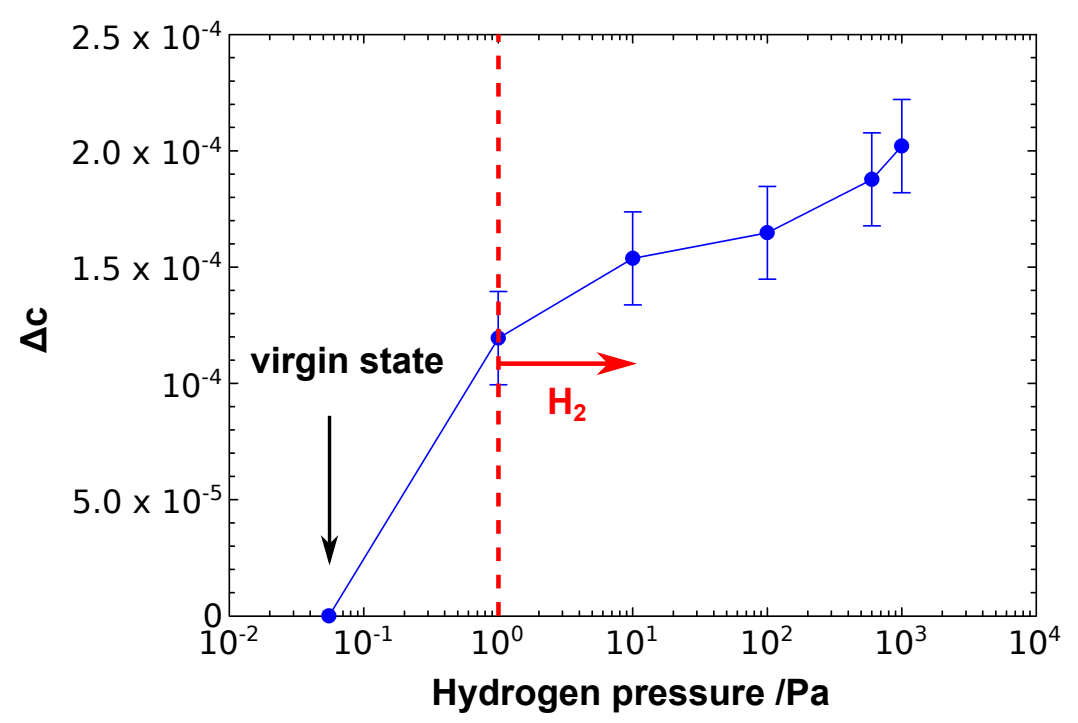

Figure 4.29.: The average change of the vacancy concentration $\Delta c_{\mathrm{V}}$ in the $\mathrm{Pd}$ film is shown as a function of the hydrogen pressure. The virgin sample was taken as a reference. $\Delta c_{\mathrm{V}}$ was calculated from $\mathrm{L}_{+}$, cf. fig. 4.28 , assuming that just $\mathrm{V}_{\mathrm{Pd}}$ contributed to the changes in the positrons diffusion length. $\Delta c_{\mathrm{V}}$ is given per atom. Adapted with permission from [132]. Copyright 2018 American Chemical Society.

state before hydrogen loading was used as a reference and therefore no change in the vacancy concentration is visible here. Introducing a hydrogen gas atmosphere of $1 \mathrm{~Pa}$ yield a change in $\mathrm{V}_{\mathrm{Pd}}$ of approximately $120 \mathrm{ppm} . \Delta c_{\mathrm{V}}$ increased further with increasing hydrogen pressure up to approximately $200 \mathrm{ppm}$ at $1000 \mathrm{~Pa}$ hydrogen.

In summary, the VEPAS studies on the $\sim 200 \mathrm{~nm} \mathrm{Pd} / \mathrm{r}-\mathrm{TiO}_{2}$ samples did not reveal changes in the bulk or the (nearby) interface vicinity of $\mathrm{r}-\mathrm{TiO}_{2}$. Thus, the concentra- 
tion of $\mathrm{V}_{\mathrm{Ti}}$ did not change significantly, or was below the detection limit of VEPAS. Other intrinsic or hydrogen related defects in $\mathrm{r}-\mathrm{TiO}_{2}$ cannot be detected by VEPAS, cf. chap. 3.4. However, in the Pd film hydrogen related changes were visible. The introduction of hydrogen yielded a decrease of the average diffusion length of the positrons in the Pd film. Therefore, new defects were created in the Pd film by the hydrogen loading which added additional open volume for positron trapping. Under the assumption that vacancies in the Pd film were responsible for the observed changes, the hydrogen induced change of the vacancy concentration calculated was in the range of $100 \mathrm{ppm}$ to $200 \mathrm{ppm}$.

\subsection{ETEM investigations on hydrogen related changes in Pd}

The VEPAS measurements show an appearance of open volume defects in the $\sim 200 \mathrm{~nm}$ $\mathrm{Pd}$ film on $\mathrm{r}-\mathrm{TiO}_{2}$ in hydrogen atmosphere. These open volume defects can either be vacancies or dislocations. While vacancies are not visible in a TEM, dislocations can be detected. Therefore, in situ studies with HR-STEM in the ETEM were performed to study the dislocation density $\rho_{\mathrm{D}}$ as a function of the hydrogen pressure as described in chap. 3.2.3.

Examples of HR-STEM images and their corresponding back transformations of the in situ investigations in hydrogen are given in fig. 4.30. Here, the HR-STEM images a), c) and e) present the middle part of the Pd film. The HR-STEM images are given for selected hydrogen pressures, namely before the hydrogen inlet at $10^{-4} \mathrm{~Pa}$ background pressure, at $10 \mathrm{~Pa}$ and at $500 \mathrm{~Pa}$. The corresponding FFT back transformation for the $\operatorname{Pd}(111)$ planes is shown in the images b), d), f). Here, the possible locations for the dislocations are indicated in red, cf. chap. 3.2.3, which is visualized in fig. 4.30b), d) and $\mathrm{f}$ ). According to the confirmed dislocations, their amount in the $\operatorname{Pd}(111)$ planes increases markedly in the case of $500 \mathrm{~Pa}$, cf. fig. 4.30f).

The dislocation density $\rho_{\mathrm{D}}^{(111)}$ was calculated in the $\operatorname{Pd}(111)$ planes in the normal direction of the film. $\rho_{\mathrm{D}}^{(111)}$ was calculated for each of five similar back transformed images and for every investigated pressure. This evaluation is summarized in fig. 4.31. Here, $\rho_{\mathrm{D}}^{(111)}$ is shown as a function of the applied hydrogen gas pressure in the ETEM. Fig. 4.31 shows the dislocation density is around $10^{15} \mathrm{~m}^{-2}$ for hydrogen pressures up to $100 \mathrm{~Pa}$. In this pressure range, no significant increase in $\rho_{\mathrm{D}}^{(111)}$ was found. An increase of the hydrogen pressure to $500 \mathrm{~Pa}$ in the ETEM, however, resulted in an increase of $\rho_{\mathrm{D}}^{(111)}$ to approximately $10^{16} \mathrm{~m}^{-2}$, cf. fig. 4.31 . Thus, at $500 \mathrm{~Pa}$ hydrogen, $\rho_{\mathrm{D}}^{(111)}$ was found to increase considerably.

In summary, the dislocation density $\rho_{\mathrm{D}}^{(111)}$ was evaluated from FFT back transformations of HR-STEM images in the middle of a $\sim 200 \mathrm{~nm} \mathrm{Pd}$ film on $\mathrm{r}-\mathrm{TiO}_{2} . \rho_{\mathrm{D}}^{(111)}$ 

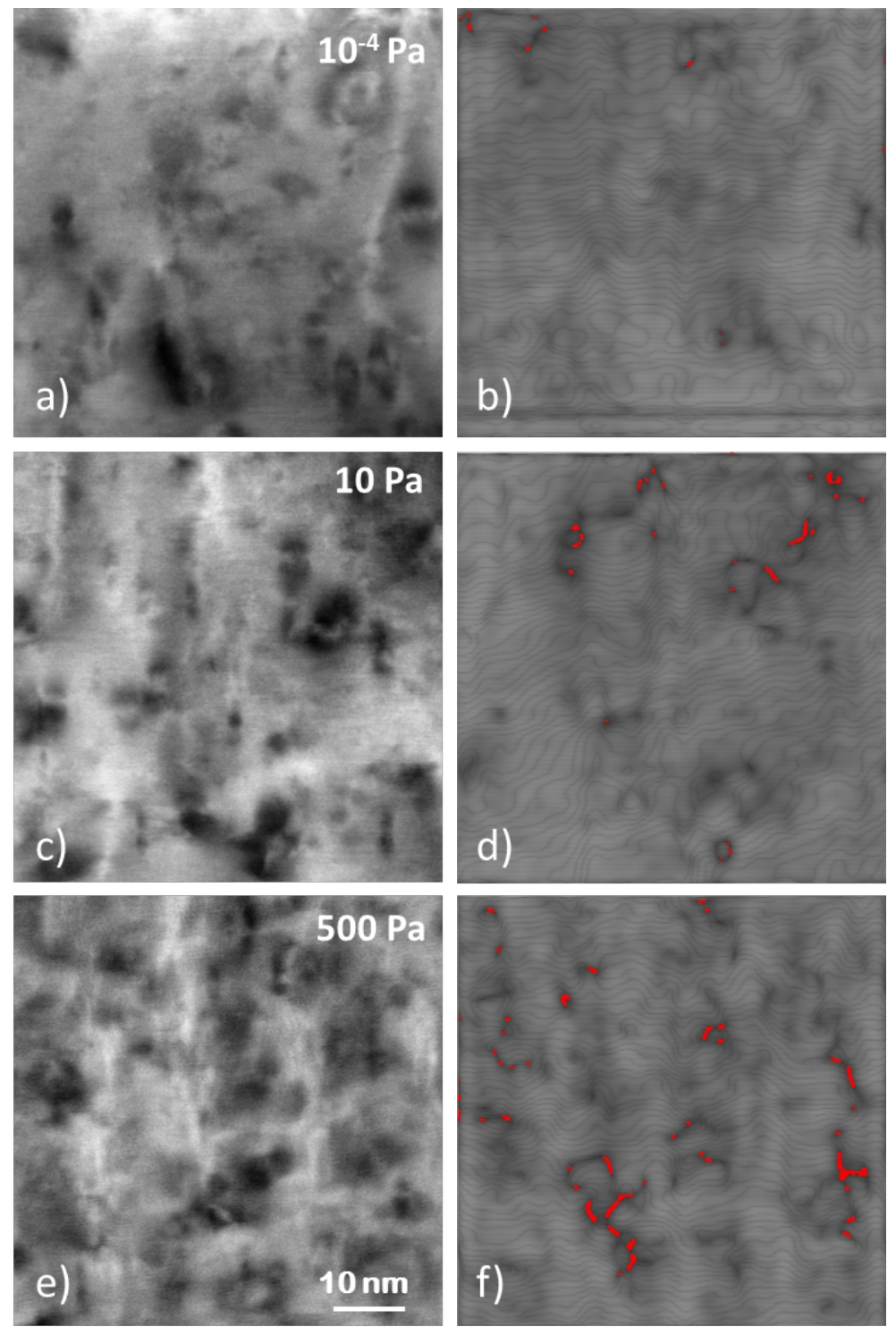

Figure 4.30.: Examples of the HR-STEM investigation of a $\sim 200 \mathrm{~nm} \mathrm{Pd}$ film on $\mathrm{r}_{-} \mathrm{TiO}_{2}$ for selected hydrogen gas pressures in the ETEM. All HR-STEM images a), c) and e) were taken in the middle of the Pd film. The corresponding FFT back transformation of the (111) planes is given in the images b), d) and f). Here, possible locations of dislocations are indicated with a red line. Before the hydrogen loading the HR-STEM investigation reveals just few dislocations, cf. image b). In hydrogen atmosphere the number of dislocation especially increases in the $500 \mathrm{~Pa}$ case as shown in images $\mathrm{f}$ ). The increase in dislocations was quantified, therefore, the dislocation density $\rho_{\mathrm{D}}$ was calculated for all investigated pressures as presented in fig. 4.31. Reprinted with permission from [132]. Copyright 2018 American Chemical Society. 


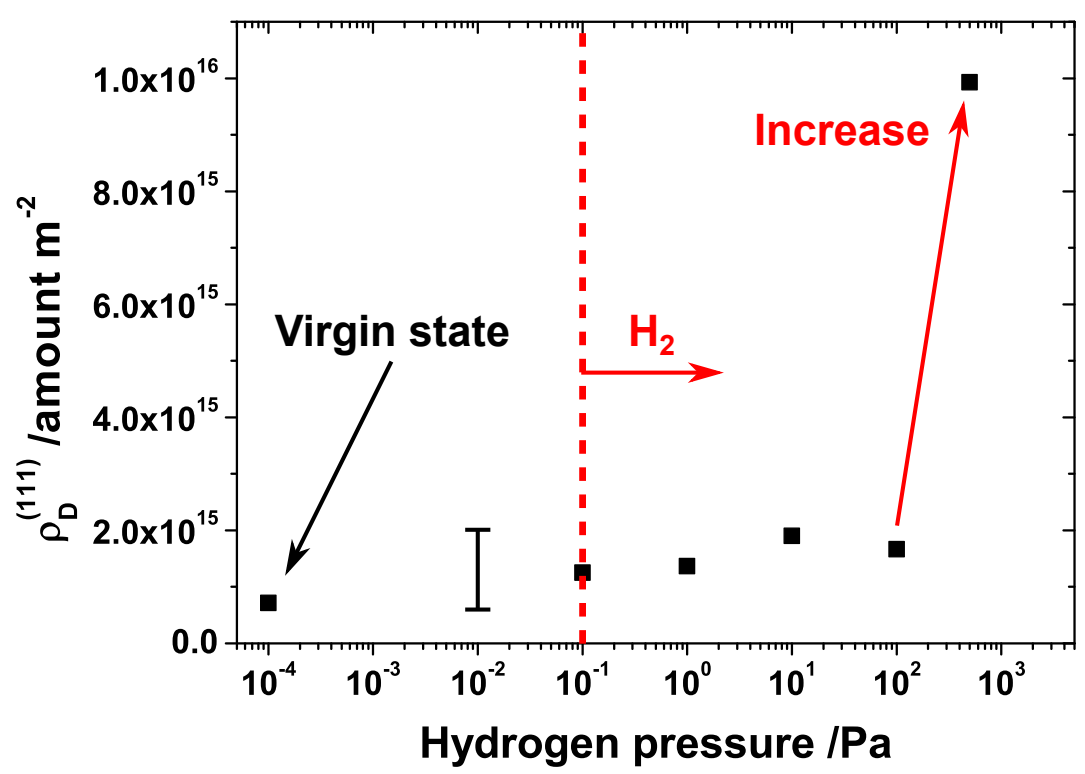

Figure 4.31.: The dislocation density $\rho_{\mathrm{D}}^{(111)}$ in the middle of the Pd film is shown as a function of the hydrogen pressure in the ETEM. For each value five different positions in the middle of the $\mathrm{Pd}$ film were investigated. Here, $10^{-4} \mathrm{~Pa}$ refers to the background pressure of the ETEM. Up to a hydrogen pressure of $100 \mathrm{~Pa} \rho_{\mathrm{D}}^{(111)}$ is approximately $10^{15} \mathrm{~m}^{-2}$. Increasing the hydrogen pressure to $500 \mathrm{~Pa}$ revealed a significant increase to $\rho_{\mathrm{D}}^{(111)} \approx 10^{16} \mathrm{~m}^{-2}$. Adapted with permission from [132]. Copyright 2018 American Chemical Society.

revealed no significant dependency on the hydrogen gas pressure in the ETEM up to $100 \mathrm{~Pa} . \rho_{\mathrm{D}}^{(111)}$ was found to be around $10^{15} \mathrm{~m}^{-2}$ in this pressure range. However, an increase to $500 \mathrm{~Pa}$ hydrogen revealed a significant increase of $\rho_{\mathrm{D}}^{(111)}$ to approximately $10^{16} \mathrm{~m}^{-2}$.

The findings of the in situ ETEM investigation of a $\sim 200 \mathrm{~nm}$ Pd film give new insights into the pressure dependent appearance of dislocations. Hence, combining the ETEM with the VEPAS measurement allows judgement about the influence of vacancies and dislocations to the average penetration depth of the positrons $\mathrm{L}_{+}$which was found to depend on the applied hydrogen gas pressure, cf. fig. 4.28. 



\section{Discussion}

This chapter discusses the obtained results with respect to the theory, cf. chap. 2, and to the literature. Based on that comparison, a final situation in the $\mathrm{Pd} / \mathrm{r}-\mathrm{TiO}_{2}$ samples in hydrogen gas environment is suggested.

In chap. 5.1 the defect disorder of the $\mathrm{r}-\mathrm{TiO}_{2}$ crystal and the orientation relationship of the deposited $\mathrm{Pd}$ film on $\mathrm{r}-\mathrm{TiO}_{2}$ are discussed. Based on this discussion, the initial state, before the in situ hydrogen loading experiments in the ETEM, of the prepared $\mathrm{Pd} / \mathrm{r}-\mathrm{TiO}_{2}$ samples is concluded.

The hydrogen induced changes that were found experimentally and theoretically are discussed with focus on bulk $\mathrm{r}-\mathrm{TiO}_{2}$, cf. chap. 5.3, on Pd, cf. chap. 5.4 and on the $\mathrm{Pd} / \mathrm{r}-\mathrm{TiO}_{2}$ interface and its vicinity, cf. chap. 5.5.

Based on that discussion a final picture of the $\mathrm{Pd} / \mathrm{r}-\mathrm{TiO}_{2}$ system in hydrogen gas atmosphere is concluded in chap. 5.6.

\subsection{Initial state of $\mathrm{Pd} / \mathrm{r}-\mathrm{TiO}_{2}$}

Based on the defect chemistry model of $\mathrm{r}-\mathrm{TiO}_{2}$ from Nowotny et al. $[24,26,30,51]$ that is presented in chap. 2.1.3 a qualitative picture of the $\mathrm{r}-\mathrm{TiO}_{2}$ crystal can be concluded after the different annealing steps performed and after the whole sample preparation.

The $\mathrm{r}-\mathrm{TiO}_{2}$ substrates were pretreated via an annealing at $T=1173 \mathrm{~K}$ and with $p\left(\mathrm{O}_{2}\right) \gtrsim 10^{5} \mathrm{~Pa}$, cf. chap. 3.1.1. The oven used did not allow for control of the pressure, thus the oxygen partial pressure could not be varied during the pretreatment. In contrast to the single crystal measurements of Nowotny et al., the crystals used are expected to contain no significant amount of $\mathrm{V}_{\mathrm{Ti}}$ (or other acceptor type defects). Thus, after the pretreatment, a low ( $n$-type) conductive crystal is expected with $\mathrm{V}_{\mathrm{O}}$ as the dominant defect species, cf. chap. 2.1.3 and curve 0 in fig. 2.9. Subsequently, the crystal was annealed in the sputter system at $1023 \mathrm{~K}$ with $p\left(\mathrm{O}_{2}\right) \approx 10^{-2} \mathrm{~Pa}$. Although this pressure range was not measured by Nowotny et al. they expect the $\mathrm{r}-\mathrm{TiO}_{2}$ crystal to be in the reduced regime. In an older work from Bak et al. [32,33,53], this pressure range was covered with (Cr-doped) $\mathrm{r}^{-\mathrm{TiO}_{2}}$ single crystals, cf. fig. 2.9. They showed that the $\mathrm{r}-\mathrm{TiO}_{2}$ crystal exhibited $n$-type character and was in the reduced regime. Thus, after the annealing in the magnetron sputter system, the crystal is of clear $n$-type nature containing $\mathrm{V}_{\mathrm{O}}$ as the dominant defect species. Although this clear qualitative classification can be drawn, a quantitative estimate about the $\mathrm{V}_{\mathrm{O}}$ concentration is not possible. After Pd film deposition, samples were cooled in a 
controlled way but not quenched. The cooling process typically reduces the defect density [3], however it is not possible to give a quantitative prediction. Thus, the $\mathrm{V}_{\mathrm{O}}$ is expected to decrease, but the sample is expected to stay $n$-type with $\mathrm{V}_{\mathrm{O}}$ as the dominant defect.

The defect equilibration in $\mathrm{r}-\mathrm{TiO}_{2}$ does not only depend on $p\left(\mathrm{O}_{2}\right)$ and $T$ but also on the time $t$ as discussed in chap. 2.1.3. Nowotny et al. [26] found that the equilibration of $\mathrm{r}-\mathrm{TiO}_{2}$ occurs mainly in the first $0.1 \mathrm{~h}$; the conductivity was found to be stable after $0.5 \mathrm{~h}$. The used time scales for the pretreatment $(\sim 1 \mathrm{~h})$ and for the Pd film deposition $(\sim 0.5 \mathrm{~h})$ are therefore sufficient to equilibrate the concentrations of $\mathrm{V}_{\mathrm{O}}$ (and in principle also $\mathrm{Ti}_{\mathrm{i}}$ ) throughout the whole $\mathrm{r}-\mathrm{TiO}_{2}$ crystal. Nowotny et al. note that for these time scales [26] the concentration of $\mathrm{V}_{\mathrm{Ti}}$ is not in equilibrium resulting in a concentration gradient from the surface to the bulk. However, the concentration of $\mathrm{V}_{\mathrm{Ti}}$ is expected to be significantly lower than that of $\mathrm{V}_{\mathrm{O}}$, especially at $p\left(\mathrm{O}_{2}\right) \approx 10^{-2} \mathrm{~Pa}$, cf. fig 2.10a. $\mathrm{V}_{\mathrm{Ti}}$ can thus be considered as minor importance in this situation. To additionally equilibrate the $\mathrm{V}_{\mathrm{Ti}}$ concentration, the duration of thermal annealing needs to be carried out for several thousand hours [26].

Considering the discussion above and the presented theory of metal-semiconductor contacts (MSC) from chap. 2.3.2, the prepared $\mathrm{Pd} / n$-type $\mathrm{r}-\mathrm{TiO}_{2}$ samples can be assumed to behave like a MSC, cf. fig. 2.15a. Thus, a Schottky barrier was established at the interface, and a band bending was present that decayed in the $\mathrm{r}-\mathrm{TiO}_{2}$ part. However, only a qualitative picture can be made since experimental values for the involved defect densities were not available. For example, the extension of the depletion layer into the $\mathrm{r}-\mathrm{TiO}_{2}$ as well as the correct height of the Schottky barrier were experimentally not addressed and were thus unknown.

Next the properties of a $200 \mathrm{~nm}$ Pd layer deposited at $1023 \mathrm{~K}$ on top of the $n$-type $\mathrm{r}-\mathrm{TiO}_{2}$ crystal are discussed.

The XRD measurements revealed a clear $\mathrm{Pd}(111)$ film growth on the $\mathrm{r}-\mathrm{TiO}_{2}(110)$ surface, cf. fig. 4.3. No other orientations were observed. The pole figure measurements, cf. fig. 4.4a, in combination with the $\phi$ scans, cf. tab. 4.3, revealed two preferred orientations of the $\mathrm{Pd}(111)$ film on the $\mathrm{r}-\mathrm{TiO}_{2}(110)$ single crystals which both exhibit the same orientation relationship: $[\overline{2} 11](111) \|[\overline{1} 10](110) \mathrm{r}-\mathrm{TiO}_{2}$ and $[0 \overline{1} 1](111) \|[001](110) \mathrm{r}-\mathrm{TiO}_{2}$. The orientation relationship was also confirmed from an HR-TEM investigation, cf. fig. 4.7a, and was summarized in fig. 4.6.

Suzuki and Souda [133] prepared a Pd film by electron beam evaporation at RT on $\mathrm{r}-\mathrm{TiO}_{2}(110)$ surfaces. They annealed the final sample, step by step, to $\left.1170 \mathrm{~K}\right]$ in UHV $\left(<2.6 \cdot 10^{-7} \mathrm{~Pa}\right)$ and investigated the orientation relationship of $\mathrm{Pd}$ with coaxial impact-collision ion scattering spectroscopy and reflection high-energy electron diffraction. They reported an orientation relationship of $[\overline{1} 2 \overline{1}](111) \|[00 \overline{1}](110) \mathrm{r}-\mathrm{TiO}_{2}$ which is not the relationship observed for the prepared Pd films in this study. That orientation relationship differs from the one found by Suzuki and Souda by a rotation of $90^{\circ}$ around the (111) axis of the $\mathrm{Pd}$ film. The main difference between their experiments and the presented experiments is the surrounding atmosphere: while 
Suzuki and Souda [133] used UHV conditions during film deposition and subsequent thermal annealing, this study used an atmosphere with $p\left(\mathrm{O}_{2}\right) \approx 10^{-2} \mathrm{~Pa}$. Why this may affect the orientation relationship is a matter for further research.

The $\operatorname{Pd}(111)$ interference was measured via XRD with $d_{111}=(2.24066 \pm 0.00002) \AA$. To calculate the normal strain $\epsilon_{111}$, this value was compared to the bulk value of Pd given by Arblaster [72] of $d_{111}^{0}=(2.2460 \pm 0.0004) \AA$, cf. tab. 2.3. The stress free lattice parameter of a thin film can deviate from the bulk value as discussed in chap. 3.5.1.3. Measurements by Harumoto et al. [111] of the stress free lattice parameter were performed on thin Pd films deposited at RT, their results suggest $d_{111}^{0}$ to be in the interval $[2.2438 \AA ; 2.2483 \AA]$. The deviation between bulk and thin film typically originated from higher defect densities present in the thin film. However, for Pd films deposited at $1023 \mathrm{~K}$ lattice defects are expected to be reduced in comparison to RT deposition as carried out by Harumoto et al.. Hence, it is expected that the deviation from the bulk value is smaller than suggested by the previously given interval of $d_{111}^{0}$. However, the measured value of $d_{111}=(2.24066 \pm 0.00002) \AA$ is clearly outside of this interval. Therefore, the sign of the normal strain does not change by considering the stress free lattice parameter of a thin Pd film; only the absolute value can change. The $\operatorname{Pd}(111)$ interference shift results in a compression of $|-0.24| \%$ of the film in normal direction. Considering the discussion of the stress free lattice parameter and the derived interval of $d_{111}^{0}$ the normal strain can be in the interval $[-0.14 \% ;-0.34 \%$ ]. Therefore, the general trend of compression in normal direction of the $\mathrm{Pd}$ films does not change by this consideration.

Using the found orientation relationship for $\mathrm{Pd}$ on $\mathrm{r}-\mathrm{TiO}_{2}(110)$, the lateral (or inplane) stresses and the normal (or out-of-plane) strain can be derived from the lattice misfit as performed in chap. 2.3.1. Lateral stresses of $\sigma_{\text {total }}\left[\operatorname{Pd}(\overline{2} 11) \| \mathrm{r}-\mathrm{TiO}_{2}(\overline{1} 10)\right]=$ $3.505 \mathrm{GPa}$ and $\sigma_{\text {total }}\left[\operatorname{Pd}(0 \overline{1} 1) \| \mathrm{r}-\mathrm{TiO}_{2}(001)\right]=2.194 \mathrm{GPa}$ were calculated and should be accompanied by a normal strain of $\epsilon_{\text {normal }}=\epsilon_{(111)}=-1.0 \%$. The measured value of $\epsilon_{(111)}$ being in the interval of $[-0.14 \% ;-0.34 \%]$ shows that the expected compression is present, however reduced. Compared to the calculated value of $-1 \%$, the compression is reduced in out-of-plane direction. This suggests a reduction of the overall stress state in the $\mathrm{Pd}$ film. This reduction can, for example, be explained by the introduction of dislocations to the $\mathrm{Pd} / \mathrm{r}-\mathrm{TiO}_{2}$ interface. The HRTEM picture, cf. fig. 4.7b, confirms this assumption. Interjectional half planes were added in the $\mathrm{Pd}$ in $<\overline{2} 11>$ direction. Thus, the stress state was significantly lowered in this direction. Even though a quantification of the lateral stress was not possible with the obtained results, the observed trend fits well to the calculated compression and its reduction due to the introduction of dislocations in the $\mathrm{Pd}$ film.

The HRTEM picture (cf. fig. 4.7b) reveals interjectional half planes in every third to fourth $\mathrm{Pd}$ row ending on the atomic Ti rows of the underlying $\mathrm{r}-\mathrm{TiO}_{2}$ crystal. Thus, a semi-coherent interface has established in $\mathrm{Pd}<2 \overline{1} \overline{1}>\| \mathrm{r}-\mathrm{TiO}_{2}<1 \overline{1} 0>$ direction. This is not surprising since a high intrinsic tensile stress state up to approximately $3.5 \mathrm{GPa}$ is 
expected in a simple misfit model in this direction, cf. 2.3.1. Hence, the incorporation of dislocations is energetically favorable to reduce the stress state and therefore the total energy of the $\mathrm{Pd} / \mathrm{r}-\mathrm{TiO}_{2}$ interface. It is interesting to note that a measurement of the (110) lattice plane distances in the $\mathrm{r}-\mathrm{TiO}_{2}$ part of the HRTEM picture (cf. fig. 4.7a) resembles only bulk values, even at the interface. Thus, the presence of the $\mathrm{Pd}$ film does not change the observed (110) lattice plane distances $\left(d_{110}\right)$. This is an important hint to justify the assumption for $\mathrm{r}-\mathrm{TiO}_{2}$ to be mechanically rigid with respect to the $\mathrm{Pd}$ film as used for the calculation of the anisotropic stress state in chap. 2.3.1. Though, no insights were obtained for the $\mathrm{r}-\mathrm{TiO}_{2}(001)$ planes, since, in the HRTEM picture, these planes are oriented in the electron beam direction.

In summary, the prepared $\mathrm{Pd} / \mathrm{r}-\mathrm{TiO}_{2}$ samples exhibited a $[\overline{2} 11](111) \|[\overline{1} 10](110) \mathrm{r}-$ $\mathrm{TiO}_{2}$ orientation relationship as depicted in fig. 4.6 with a semi-coherent interface along $\mathrm{Pd}<2 \overline{1} \overline{1}>\| \mathrm{r}-\mathrm{TiO}_{2}<1 \overline{1} 0>$ that exhibits dislocations in every third to fourth $\mathrm{Pd}(\overline{1} 11)$ plane. The $\mathrm{r}-\mathrm{TiO}_{2}$ crystal is expected to exhibit $n$-type conductivity with $\mathrm{V}_{\mathrm{O}}$ as the dominant defect species. The EELS measurements were thus performed in $\mathrm{Pd}<01 \overline{1}>$ and $\mathrm{r}-\mathrm{TiO}_{2}<001>$ direction on these samples.

As remarked by Brydson et al. [35] the shape of the Ti $L$ edge does not depend on the crystal direction for $\mathrm{r}-\mathrm{TiO}_{2}$.

Possible improvements for a future sample preparation include that the pretreatment should be carried out at $T$ and $p\left(\mathrm{O}_{2}\right)$ chosen to reach the wanted properties of the $\mathrm{r}-\mathrm{TiO}_{2}$ crystal. For this an oven is needed that allows control of the temperature and oxygen partial pressure in the needed ranges. For example, it may be useful to chose the same conditions present during the deposition process of Pd. Further, the duration of the pretreatment can be extended to $2 \mathrm{~h}$ to ensure an equilibrium state (in kinetic regime I) of the whole $\mathrm{r}-\mathrm{TiO}_{2}$ crystal, cf. chap. 2.1.3. In addition, a fast cooling of the $\mathrm{Pd} / \mathrm{r}-\mathrm{TiO}_{2}$ samples after the $\mathrm{Pd}$ film deposition in the magnetron sputter system can be advantageous to keep the defect concentration present in $\mathrm{r}-\mathrm{TiO}_{2}$ at the elevated temperature. Also, carrying out the Pd deposition without heating (at all) keeps the $\mathrm{r}-\mathrm{TiO}_{2}$ crystal in the state chosen with the pretreatment conditions. Thus, the $\mathrm{Pd} / \mathrm{r}-\mathrm{TiO}_{2}$ interface and its corresponding properties (e.g. Schottky barrier) are established in a $\mathrm{r}-\mathrm{TiO}_{2}$ crystal with homogeneously distributed defects and therefore uniform properties. Though, this yields to a $\mathrm{Pd}$ film with a nanocrystalline grain structure. 


\section{2. $\mathrm{Pd} / \mathrm{r}-\mathrm{TiO}_{2}$ equilibration with the hydrogen gas environment in the ETEM}

In this section the equilibration between hydrogen and the prepared $\mathrm{Pd} / \mathrm{r}-\mathrm{TiO}_{2}$ samples in the ETEM is discussed. The samples were equilibrated for $1 \mathrm{~h}$ at the corresponding hydrogen gas pressure without the presence of the TEM's electron beam, cf. chap. 3.2.2. Following is a discussion on the consideration of the necessary equilibration time of the $\mathrm{Pd} / \mathrm{r}-\mathrm{TiO}_{2}$ system on the bases of diffusion and gas sensing measurements.

The hydrogen diffusion in $\mathrm{r}-\mathrm{TiO}_{2}$ was studied by Johnson et al. [62], cf. chap. 2.1.4.1, for a crystal being in the oxidized or in the poorly reduced regime. A strong anisotropy was found with respect to the $c$-axis: $D_{\perp c} \ll D_{\| c}$. Therefore, the hydrogen diffusion in $\mathrm{r}-\mathrm{TiO}_{2}<110>$ direction is predicted to be very slow, cf. chap. 2.1.4.1, suggesting a diffusion barrier present in this direction.

Diffusion of intrinsic defects in $\mathrm{r}-\mathrm{TiO}_{2}$ depends on the defect equilibration as studied by Nowotny et al. [24], cf. chap. 2.1.3 and fig. 2.12. Hydrogen diffusion data for reduced $n$-type $\mathrm{r}-\mathrm{TiO}_{2}$ such as in this study are not available in the literature. Thus the influence of a diffusion limited process is difficult to estimate. However, the EELS measurements on $\mathrm{Pd} / \mathrm{r}-\mathrm{TiO}_{2}$ performed in the ETEM revealed a clear influence of hydrogen in the close within $2 \mathrm{~nm}$ vicinity of the interface. If diffusion is slow, the extension of this layer may increase with time. This was not studied but it is not expected that a possible diffusion limit changes the quality of the observed chemical shift in the EELS experiments.

Measurements of the $I-V$ characteristics on comparable $\mathrm{Pd} / \mathrm{r}-\mathrm{TiO}_{2}$ systems in hydrogen environment performed by Yamamoto et al. [10] and Kobayashi et al. [11] suggest a fast equilibration within a few minutes, cf. chap. 2.3.2.1. Thus, the possible diffusion barrier does not influence the hydrogen sensing effect. This suggests an interface or near-interface effect. Hence, the used timescales for hydrogen equilibration were sufficient to study a possible gas sensing effect in the vicinity of the $\mathrm{Pd} / \mathrm{r}-\mathrm{TiO}_{2}$ interface.

In addition, it is important to note that all the addressed measurements from literature were performed on bulk samples. This is an important difference to the dimension of the TEM lamella that were used. As discussed in chap. 5.1 and shown in fig. 4.6 the EELS investigations were performed in $<001>$ direction, cf. fig. 4.7. Thus, a lot of free $\mathrm{r}-\mathrm{TiO}_{2}(001)$ surface was present. Since the diffusion parallel to the $c$-axis is significantly faster, cf. chap. 2.1.4.1, the hydrogen could diffuse from the $\{001\}$ surfaces into the $\mathrm{r}-\mathrm{TiO}_{2}$ part of the TEM lamella and equilibrate from this sides. This requires the atomic hydrogen to spill-over from Pd onto the (001) surface. For this process no data was available and therefore it was not possible to judge about the presence of such a spill-over process. In addition, the electron beam might have increased the diffusivity as discussed by Chen et al. [67], cf. chap. 2.1.4. Also radiolysis [96] of $\mathrm{H}_{2}$ by the electron beam can supply atomic hydrogen to the (001) surface directly from 
the hydrogen gas environment.

\subsection{Hydrogen induced changes within 'bulk' $\mathrm{r}-\mathrm{TiO}_{2}$}

The "bulk" region of $\mathrm{r}-\mathrm{TiO}_{2}$ was studied with VEPAS, EELS and DFT calculations in different hydrogen environments.

VEPAS studies were performed on $200 \mathrm{~nm} \mathrm{Pd} / \mathrm{r}-\mathrm{TiO}_{2}$ samples, cf. chap. 4.7: The hydrogen gas pressure was varied from $1 \mathrm{~Pa}$ to $1000 \mathrm{~Pa}$ and the $S$ parameter was evaluated as a function of the positrons energy $E$ at every pressure step, cf. fig. 4.27. The performed VEPAS studies did not reveal significant changes in $\mathrm{r}-\mathrm{TiO}_{2}(E>$ $13 \mathrm{keV}$ ) as a function of the hydrogen gas pressure. PAS can mainly detect changes in the $\mathrm{V}_{\mathrm{Ti}}$ concentration in $\mathrm{r}-\mathrm{TiO}_{2}$ as explained in chap. 3.4. Therefore, the performed VEPAS studies did not reveal any significant concentration change of $\mathrm{V}_{\mathrm{Ti}}$ due to the hydrogen loading. The prepared $\mathrm{r}-\mathrm{TiO}_{2}$ crystals were expected to be in the reduced regime and thus exhibit $n$-type conductivity with $\mathrm{V}_{\mathrm{O}}$ being the dominant defect [30], cf. chap. 5.1. Thus, $\mathrm{Ti}_{\mathrm{i}}$ is not expected to be present in any significant concentration in the produced samples. Combining the VEPAS results and the considerations of the sample preparation, it can be concluded that Ti related defects do not play any significant role in the hydrogen interaction of the prepared $\mathrm{Pd} / \mathrm{r}-\mathrm{TiO}_{2}$ samples.

The performed DFT calculations, cf. chap. 4.4, therefore focused on oxygen and hydrogen related defects in $\mathrm{r}-\mathrm{TiO}_{2}$, namely $\mathrm{V}_{\mathrm{O}}, \mathrm{H}_{\mathrm{i}}$ and $\mathrm{H}_{\mathrm{O}}$. An investigation of the thermodynamics revealed that the binding energy of $\mathrm{H}_{\mathrm{O}}$ becomes negative in a broad range of $\mu_{e}$ in the band gap, cf. fig. 4.15. This results in a driving force to form $\mathrm{H}_{\mathrm{O}}$ from $\mathrm{V}_{\mathrm{O}}$ and $\mathrm{H}_{\mathrm{i}}$ in the material for this range of $\mu_{e}$. A thermodynamic model was applied in which the concentrations of the studied defects were calculated as a function of $\mu_{\mathrm{H}}$. For that purpose, $\mu_{\mathrm{O}}$ was fixed at $-4.35 \mathrm{eV}$, which corresponds to $\mathrm{r}-\mathrm{TiO}_{2}$ being in the reduced regime and $\mathrm{V}_{\mathrm{O}}$ being the dominant defect without the presence of hydrogen, cf. fig. 4.16. The thermodynamic model yielded the neutrally charged $\mathrm{H}_{\mathrm{O}}\left(\mathrm{H}_{\mathrm{O}}^{0}\right)$ being the dominant defect in the bulk in a broad range of $\mu_{\mathrm{H}}$, cf. fig. 4.17a. $\mathrm{A} \mathrm{H}_{\mathrm{O}}^{0}$ concentration of approximately $3 \cdot 10^{-4}$ per oxygen site was calculated for $\mu_{\mathrm{H}}=-0.14 \mathrm{eV}(\triangleq 10 \mathrm{~Pa})$.

The 'bulk' of $\mathrm{r}-\mathrm{TiO}_{2}$ was also studied with the in situ EELS experiments, cf. chap. 4.3. The fine structure splitting $\mathrm{d} E\left(b^{\prime}-a\right)$ was evaluated at least $30 \mathrm{~nm}$ away from the $\mathrm{Pd} / \mathrm{r}-\mathrm{TiO}_{2}$ interface in the initial state, at $1 \mathrm{~Pa}$ and $10 \mathrm{~Pa}$ hydrogen, cf. fig. 4.9. No influence of $\mathrm{d} E\left(b^{\prime}-a\right)$ was found on the hydrogen gas pressure. An energy splitting of $2.8 \mathrm{eV}$ was measured. Brydson et al. [35] reported an energy splitting of $(2.86 \pm 0.1) \mathrm{eV}$ for a $\mathrm{r}-\mathrm{TiO}_{2}$ single crystal. Thus, the found value of $2.8 \mathrm{eV}$ for $\mathrm{d} E\left(b^{\prime}-a\right)$ fits nicely to known values for $\mathrm{r}-\mathrm{TiO}_{2}$. Therefore, no influence of hydrogen on 'bulk' $\mathrm{r}-\mathrm{TiO}_{2}$ was found by the in situ EELS experiments. 
In summary, titanium related defects in bulk $\mathrm{r}-\mathrm{TiO}_{2}$ are not expected to be present or change in the hydrogen environment. However the DFT calculations predict that $\mathrm{H}_{\mathrm{O}}$ is the dominant defect in a large $\mu_{\mathrm{H}}$ (or pressure) range if the $\mathrm{r}-\mathrm{TiO}_{2}$ is sufficiently reduced beforehand. Therefore, the dominant defect in the bulk $\mathrm{r}-\mathrm{TiO}_{2}$ in a hydrogen environment is expected to be $\mathrm{H}_{\mathrm{O}}$ rather than $\mathrm{V}_{\mathrm{O}}$.

Instability and uncertainty The EELS measurement performed on the 'bulk' material before the hydrogen introduction and at $1 \mathrm{~Pa}$ revealed a larger uncertainty and a stronger scattering than that performed at $10 \mathrm{~Pa}$ hydrogen, cf. fig. 4.9. A clear origin of this behavior is difficult to address. It is, though, a typical behavior observed for different measurements. Many parameters are contributing to the $\mathrm{Ti} L$ spectra, as discussed in chap. 3.3.1. Since the EEL spectra were affected by all instabilities (internal and external) from the TEM and from its surroundings, these factors are considered to explain the additional scattering and thus the enlarged uncertainty as well as the observed scattering. But, the parameters of the EELS acquisition were optimized to minimize these influences. The EELS measurements performed at $10 \mathrm{~Pa}$ were taken with these optimized parameters that are summarized in appendix A.5.

It is important to note that the evaluation via the MATLAB script did not add further scattering. To ensure that, all fits of the single EEL spectra were checked manually in the evaluation process. However, clearly small energy changes could be detected with the presented EELS methodology.

\subsection{Hydrogen induced changes within the Pd film}

The Pd films were studied with VEPAS and HR-STEM in situ for different hydrogen gas pressures. Changes in the defect content were observed and are discussed in the following.

With VEPAS, the $S(E)$ parameter of a $\sim 200 \mathrm{~nm} \mathrm{Pd} / \mathrm{r}-\mathrm{TiO}_{2}$ sample was measured as a function of the hydrogen gas pressure, cf. chap. 4.7. An increase of the $S(E)$ parameter in the $\mathrm{Pd}$ film revealed additional open volume defects appearing in the presence of hydrogen, cf. fig. 4.27. These open volume defects can either be vacancies or dislocations. VEPAS alone cannot distinguish between those defects. Hence, complementary in situ HR-STEM investigations were carried out on a similar Pd film in the ETEM, cf. chap. 4.8. Here, the dislocation density $\rho_{\mathrm{D}}^{(111)}$ in the $\operatorname{Pd}(111)$ planes in normal direction was investigated as a function of the applied hydrogen gas pressure, cf. fig. 4.31. No significant increase in $\rho_{\mathrm{D}}^{(111)}$ up to hydrogen gas pressures of $100 \mathrm{~Pa}$ was revealed. In this pressure range, $\rho_{\mathrm{D}}^{(111)}$ was found to be in the order of $10^{15} \mathrm{~m}^{-2}$. Only at a hydrogen gas pressure of $500 \mathrm{~Pa}$ was a significant increase of $\rho_{\mathrm{D}}^{(111)}$ to approximately $10^{16} \mathrm{~m}^{-2}$ observed. The dislocation density of a well-annealed pure metal is in the order of $10^{10} \mathrm{~m}^{-2}$ [134, p. 213]. Therefore, the dislocation density is approximately five orders of magnitude higher than in the initial condition of the 
Pd film. This clarifies the higher defect and dislocation density, respectively, after the sputtering deposition. The hydrogen loading at $500 \mathrm{~Pa}$ yielded in a dislocation density of $10^{16} \mathrm{~m}^{-2}$. According to the calculation in chap. 2.2 at $500 \mathrm{~Pa}$, a lateral (in-plane) stress $\sigma \approx|-200| \mathrm{MPa}$ builds up in the Pd film. It is likely that this hydrogen induced stress is decreased by the introduction of additional dislocations.

The hydrogen pressure dependency of the dislocation density can be used to interpret the obtained VEPAS data: The observed decrease of the average positron diffusion length $\mathrm{L}_{+}$with increasing hydrogen gas pressure is shown again in fig. 5.1. In the as

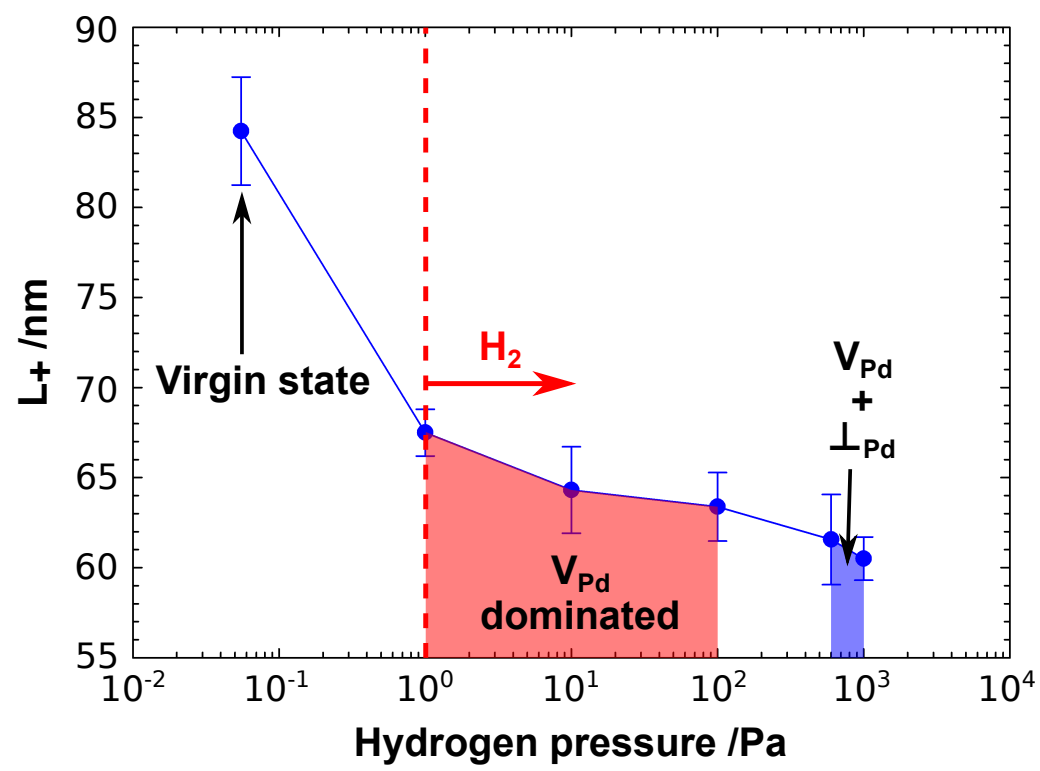

Figure 5.1.: The average diffusion length $\mathrm{L}_{+}$of the positrons in the $\mathrm{Pd}$ film is shown as a function of the applied hydrogen gas pressure, cf. fig. 4.28. Combining the in situ HR-STEM study and the VEPAS experiments suggests that vacancies $\mathrm{V}_{\mathrm{Pd}}$ dominate the change of $\mathrm{L}_{+}$up to a hydrogen gas pressure of $100 \mathrm{~Pa}$. At and above $500 \mathrm{~Pa}$ hydrogen the HR-STEM study suggested a significant increase in the dislocation density in the normal (111) planes of the $\mathrm{Pd}$ film. Therefore both dislocations $\perp_{\mathrm{Pd}}$ and $\mathrm{V}_{\mathrm{Pd}}$ can be responsible for the decreasing $\mathrm{L}_{+}$. In collaboration with Jakub Č́ižek.

prepared (or virgin) state, $\mathrm{L}_{+}$is approximately $85 \mathrm{~nm}$. $\mathrm{L}_{+}$decreases with hydrogen to approximately $67 \mathrm{~nm}$ at $1 \mathrm{~Pa}$ and further to approximately $60 \mathrm{~nm}$ at $1000 \mathrm{~Pa}$.

Based on the in situ HR-STEM investigations of the Pd film in the ETEM, it is reasonable to assume the following: The observed creation of additional open volume defects in a hydrogen atmosphere and the accompanying decrease of $\mathrm{L}_{+}$is due to the appearance of vacancies, up to a pressure of $100 \mathrm{~Pa}$, cf. red area in fig. 5.1. At higher hydrogen gas pressures, the formation of dislocations can create new open volume in addition to the vacancies, cf. blue area in fig. 5.1. Hence, the calculation of the 
vacancy concentration is reasonable up to $100 \mathrm{~Pa}$, cf. fig. 4.29. Additional vacancies of approximately $100 \mathrm{ppm}$ were created in the $\mathrm{Pd}$ film at this pressure.

In summary, combining ETEM and VEPAS investigations on $\sim 200 \mathrm{~nm} \mathrm{Pd} / \mathrm{r}-\mathrm{TiO}_{2}$ samples, it was revealed that, between $1 \mathrm{~Pa}$ and $100 \mathrm{~Pa}$ hydrogen, new vacancies were formed. The increase of the vacancy concentrations in this pressure range was found to be on the order of $100 \mathrm{ppm}$. At $500 \mathrm{~Pa}$ hydrogen, dislocations formed in the $\mathrm{Pd}$ films. Thus, both additional vacancies and additional dislocations were present in this pressure range, cf. fig. 5.1.

\subsection{Hydrogen induced changes at the $\mathrm{Pd} / \mathrm{r}-\mathrm{TiO}_{2}$ interface and in its vicinity}

Hydrogen induced changes at the $\mathrm{Pd} / \mathrm{r}-\mathrm{TiO}_{2}$ interface and in its vicinity were investigated with VEPAS, in situ EELS experiments and DFT calculations.

The performed VEPAS studies revealed no change of the $S$ parameter in the interface vicinity at any hydrogen gas pressure, cf. fig. 4.7. Therefore, the $\mathrm{V}_{\mathrm{Ti}}$ concentration did not reveal significant changes and, as for in the bulk, Ti related defects are not expected in the interface vicinity.

DFT calculations based on the thermodynamic model were performed on the $\mathrm{Pd} / \mathrm{r}$ $\mathrm{TiO}_{2}$ system in an electrostatic picture. The concentrations of $\mathrm{V}_{\mathrm{O}}, \mathrm{H}_{\mathrm{i}}$ and $\mathrm{H}_{\mathrm{O}}$ in their different charge states were studied at the $\mathrm{Pd} / \mathrm{r}-\mathrm{TiO}_{2}$ interface as a function of $\mu_{\mathrm{H}}$ with $\mu_{\mathrm{O}}$ fixed at $-4.35 \mathrm{eV}$, cf. fig. $4.17 \mathrm{~b}$. As for the bulk $\mathrm{r}-\mathrm{TiO}_{2}$, the dominant defect was $\mathrm{H}_{\mathrm{O}}$ but in the positive charge state. In addition, the $\mathrm{H}_{\mathrm{O}}^{+}$concentration is far higher than in the bulk $\mathrm{r}-\mathrm{TiO}_{2}$. $\mathrm{A} \mathrm{H}_{\mathrm{O}}^{+}$concentration of approximately 0.7 per oxygen site results for $\mu_{\mathrm{H}}=-0.14 \mathrm{eV}(\triangleq 10 \mathrm{~Pa})$. But, it can reach defect concentrations of one per oxygen site for higher $\mu_{\mathrm{H}}$. According to the interface calculations, cf. fig. $4.17 \mathrm{~b}$, the $\mathrm{H}_{\mathrm{O}}^{+}$concentration depends strongly on $\mu_{\mathrm{H}}$. Therefore, changing $\mu_{\mathrm{H}}$ and the hydrogen gas pressure, respectively, significantly changes the $\mathrm{H}_{\mathrm{O}}^{+}$concentration present at the interface. This finding can explain the strong dependency of the changes found with EELS on the hydrogen partial pressure. In the partial DoS of the $\mathrm{H}_{\mathrm{O}}$ defect, cf. chap. 4.4.3, the defect exhibit Ti $d$ states that shift in the region between the $t_{2 g^{-}}$and $e_{g}$-like states. This shift was found to be a common phenomenon for all charge states of $\mathrm{H}_{\mathrm{O}}$, see fig. 4.21, 4.22 and 4.23. Since DoS and EELS are closely related, this DoS shift yields a qualitative explanation for the changes in the Ti $L$ ELNES found in the interface vicininty at $10 \mathrm{~Pa}$ hydrogen.

$\mathrm{R}-\mathrm{TiO}_{2}$ in the $\mathrm{nm}$ vicinity of the $\mathrm{Pd} / \mathrm{r}-\mathrm{TiO}_{2}$ interface was studied with the in situ EELS experiments, cf. chap. 4.3. Here, $\mathrm{d} E\left(b^{\prime}-a\right)$ did not reveal any changes up to a hydrogen gas pressure of $1 \mathrm{~Pa}$, cf. fig. 4.10. Increasing the hydrogen gas pressure to $10 \mathrm{~Pa}$ yielded to a decrease of $\mathrm{d} E\left(b^{\prime}-a\right)$ in the close $(2 \mathrm{~nm})$ vicinity of the interface 
by approximately $|-150| \mathrm{meV}$, cf. fig. 4.10c. The decrease of $\mathrm{d} E\left(b^{\prime}-a\right)$ was found in different places in the interface vicinity, cf. fig. 4.11. This finding suggests that the interface near $\mathrm{r}-\mathrm{TiO}_{2}$ region behaved similarly in a hydrogen environment and that a homogeneous change in the close vicinity of the whole $\mathrm{Pd} / \mathrm{r}-\mathrm{TiO}_{2}$ interface appeared. This fits well to the observations by Cerchez et al. [12] that suggested a homogeneous lowering of the Schottky barrier height of the $\mathrm{Pt} / \mathrm{np}-\mathrm{TiO}_{2}$ system in hydrogen. The decrease in $\mathrm{d} E\left(b^{\prime}-a\right)$ was reinterpreted by considering difference spectra between a changed and an unchanged EEL spectrum, cf. fig. 4.12. The EEL difference spectra revealed a loss of EELS intensity at the $b^{\prime}$ white line side and an increase of intensity on the $b$ white line side. Since EELS is related to the underlying DoS, these results suggested a change in the DoS close to the interface. This finding was interpreted as a shift of states from the $b^{\prime}$ region to the $b$ region of the white line and, therefore, within the $e_{g}$-like states of the DoS, cf. fig. 2.6, being on the order of $1 \mathrm{eV}$. Similar chemical shifts of states were found in DFT calculations as already discussed. Hence, combining the theoretical and experimental findings, the presence of defects in $\mathrm{r}-\mathrm{TiO}_{2}$ in the close vicinity of the $\mathrm{Pd} / \mathrm{r}-\mathrm{TiO}_{2}$ interface in a hydrogen atmosphere with $10 \mathrm{~Pa}$ is concluded. Hence, the presence of this defects results in the observed shift of states.

The presented EELS methodology, cf. chap. 3.3.2, thus needs high defect concentrations to be applicable but can reveal changes in the $100 \mathrm{meV}$ range in EEL spectra with a local resolution of $1 \mathrm{~nm}$.

As already mentioned, the DFT calculations based on the thermodynamic model confirmed a strong dependency of the $\mathrm{H}_{\mathrm{O}}^{+}$concentrations at the $\mathrm{Pd} / \mathrm{r}-\mathrm{TiO}_{2}$ interface on the hydrogen chemical potential and pressure, respectively. This is in qualitative agreement with the experimental finding on the hydrogen gas pressure dependency in the EEL spectra: the concentration of the defect(s) was sufficient to be detectable with EELS at $10 \mathrm{~Pa}$ hydrogen, but it was not at $1 \mathrm{~Pa}$. Following the argument of a strong dependency of the DoS changes on the hydrogen chemical potential, it was of interest to test in hydrogen gas pressure above $10 \mathrm{~Pa}$. Therefore, the in situ EELS experiments were also applied at $100 \mathrm{~Pa}$ hydrogen. However, the TEM lamella exhibited strong spatial drift which could not be compensated for. Strong spatial drift results in a strong interface movement within the timescale of the taken SI pattern, see fig. 3.14. This blurs out any interface effects and, therefore, no useful measurements could be performed at this pressure range. The origin of the strong spatial drift is unclear.

The $\mathrm{H}_{\mathrm{O}}^{+}$concentration that was detectable with the presented EELS method was estimated via the relative CoG shift, cf. chap. 4.5. This was done by comparing the experimentally measured CoG shift, cf. chap. 4.3.1, with the theoretically calculated one, cf. chap. 4.4.4. $\mathrm{H}_{\mathrm{O}}^{+}$concentrations in the order of $10 \%$ per oxygen site were calculated from that comparison, cf. chap. 4.5. Such high values for the defect concentrations are already at the limit of the presented comparison since defect-defect interaction is possible, but this interaction was not considered in the DFT calculations. Hence, the calculated concentration values have to be taken with caution as the calculations had limitations. The presented model was sensitive to changes of the 
integration boundaries that separated the $t_{2 g}$ from the $e_{g}$ part. The observed trend of the CoG shifts could be driven in the positive, as well as in the negative, direction which indicates the instability of the theoretically calculated CoG shifts with the used integration windows. To solve this issue, a better understanding is necessary in how DoS is translated in EELS. For example, a broadening of the DoS could be applied to achieve a better comparison to the experiment. Also, core-hole effects could be taken into account. In general, coming from DoS to EEL spectra needs the calculation of the atomic oscillator strength $(d f / d E)$ and the density of final states in the electron transition $N(E)$ [40], cf. eq. 2.1. For the comparison, it was assumed that the electron intensity measured with EELS $J_{C}(E) \propto \operatorname{DoS}(E)$ and $\frac{J_{C}(E)}{\operatorname{DoS}(E)}=$ constant, respectively. This simplified assumption partially neglects the non-linearity in $(d f / d E)$ and $N(E)$ and the excited state in $N(E)$ including the corresponding relaxations, such as corehole effects. The simulation of $L$ edge spectra, though, is challenging even today [40]. Therefore a direct comparison as carried out can only yield a rough estimate of the order of magnitude. More precise values for defect concentrations require a suitable simulation of EEL Ti $L$ spectra. From these, a better suited CoG shift could be calculated and compared to the EELS measurements. Hence, a systematical and challenging to estimate uncertainty belongs to the calculated defect concentrations that is neglected for simplicity.

During hydrogen loading, it was recognized that cavities - regions in $\mathrm{r}-\mathrm{TiO}_{2}$ that were thinner than the surrounding $\mathrm{r}-\mathrm{TiO}_{2}$ - formed at the $\mathrm{Pd} / \mathrm{r}-\mathrm{TiO}_{2}$ interface and its vicinity, cf. chap. 4.6. Although the cavities were not studied in detail, some conclusions can be drawn. A few cavities were found already in the initial state of the TEM lamella. However after the hydrogen loading procedure, significantly more cavities had appeared, even in regions where the electron beam was not present during the hydrogen loading. This suggests that the presence of hydrogen 'triggers' the formation of those cavities. The observed reduction of the thickness by approximately half in the cavity region, cf. fig. $4.26 \mathrm{~b}$, suggests a corresponding volume reduction of approximately half in comparison to the surrounding $\mathrm{r}-\mathrm{TiO}_{2}$ matrix. Since $\mathrm{Ti}$ is not expected to leave the sample, only an oxygen loss seems reasonable. Taking the ionic radii of $\mathrm{Ti}(+4) r_{\mathrm{Ti}}$ and $\mathrm{O}(-2) r_{\mathrm{O}}$ in $\mathrm{r}^{-\mathrm{TiO}_{2}}[27, \mathrm{p} .61]$ a rough estimate of the oxygen loss $x$ with respect to the formula unit $\mathrm{TiO}_{2-x}$ was made:

$$
x=\frac{1 / 2 \cdot V\left(\mathrm{TiO}_{2}\right)}{V(\mathrm{O})}=\frac{1 / 2 \cdot\left(4 / 3 \pi r_{\mathrm{Ti}}^{3}+2 \cdot 4 / 3 \pi r_{\mathrm{O}}^{3}\right)}{4 / 3 \pi r_{\mathrm{O}}^{3}} \approx 1.05
$$

Thus, based on this estimate which neglects changes in density and the influence of stresses, the volume reduction in the cavities yields to a formula unit of $\mathrm{TiO}_{0.95}$. Based on the phase diagram of titanium oxide, cf. fig. 2.1, this suggests the presence of $\mathrm{TiO}_{1}$ since no other stoichiometric phase does exist in the range of a $\mathrm{Ti} / \mathrm{O}=1$ ratio. $\mathrm{A}$ possible incorporation of hydrogen that may change the stoichiometry is also unknown. As already stated in chap. 4.6, the cavities do not contribute to the EELS signal with 
the used acquisition parameters. Although, the observed extension of the cavities in $z$ direction $(\sim 2 \mathrm{~nm})$ was found to be similar to the observed extension within the in situ EELS experiments $(\lesssim 2 \mathrm{~nm})$. If a high defect concentration is present in the close vicinity of the $\mathrm{Pd} / \mathrm{r}-\mathrm{TiO}_{2}$ interface in the $\mathrm{r}-\mathrm{TiO}_{2}$ part - as suggested by the in situ EELS experiments - this results in sub-stoichiometry in this region. Substoichiometry is a driving force for the formation of titanium oxide phases below the 1:2 stoichiometry. In this picture, the cavities may be considered as nucleation sites or possibly nuclei of a sub-stoichiometric titanium oxide phase. The presented ideas are merely hypotheses and more detailed studies are needed. These cavities are of great interest. Especially if the inhomogeneous interface change discovered on the $\mathrm{Pd} / \mathrm{np}$ $\mathrm{TiO}_{2}$ system observed by Cerchez et al. [12] and Strungaru et al. [13] is considered. Here, the observed cavities can yield a possible explanation for the decreasing diode area and the increasing resistive area of $\mathrm{Pd} / \mathrm{np}-\mathrm{TiO}_{2}$ in hydrogen as found by Cerchez et al. [12] or for the formation of conductive filaments as suggested by Strungaru et al. [13]. Measurements of the Ti $L$ edge in the cavity region in the future can help to reveal a possible phase change since the shape of the Ti $L$ ELNES strongly depends on the present titanium oxide phase [22]. The reduced thickness, though, of the cavities may be a limiting factor for the $\mathrm{S} / \mathrm{N}$ ratio for acquiring proper EEL spectra.

In summary, the DFT calculations suggest $\mathrm{H}_{\mathrm{O}}^{+}$to be the dominant defect at the $\mathrm{Pd} / \mathrm{r}-\mathrm{TiO}_{2}$ interface and its vicinity in reduced $\mathrm{r}-\mathrm{TiO}_{2}$ in a hydrogen gas environment. In the simulated partial DoS of $\mathrm{H}_{\mathrm{O}}^{+}$, states were shifted in the region between the $t_{2 g^{-}}$ and $e_{g}$-like states. With the in situ EELS experiments, a similar shift of states of approximately $1 \mathrm{eV}$ was revealed in $\mathrm{r}-\mathrm{TiO}_{2}$ in a $2 \mathrm{~nm}$ vicinity of the $\mathrm{Pd} / \mathrm{r}-\mathrm{TiO}_{2}$ interface. The shift was found in many places, suggesting a common (or homogeneous) change of the interface and its vicinity. Hence, combining both observations the accumulation of $\mathrm{H}_{\mathrm{O}}^{+}$is concluded in $\mathrm{r}-\mathrm{TiO}_{2}$ in the $\mathrm{Pd} / \mathrm{r}-\mathrm{TiO}_{2}$ interface vicinity. High defect concentrations are expected to measure the observed changes in EELS. A comparison of theory and experiment allows a rough estimate of the defect concentration being in the order of $10 \%$ per oxygen site. The suggestion for the final situation is depicted in fig. 5.2 .

Instability and uncertainty A typical feature was the increased uncertainty at $z$ close to the interface. Two components are held responsible. One is the electron beam that crosses from the $\mathrm{r}-\mathrm{TiO}_{2}$ side to the $\mathrm{Pd}$ side. This decreases the signal-to-noise ratio in the single EEL spectra and thus increases the data scattering and with it the uncertainty. The second component is the interface. It can deviate from the atomic flatness in the length of $20 \mathrm{~nm}$ to $25 \mathrm{~nm}$ used in the SI pattern parallel to the interface, cf. $y$-direction in fig. 3.14. Therefore, the averaging in $y$-direction can blur the effect in the averaging process, cf. the blue box in fig. 3.14. The gained uncertainty proves the advantage of the chosen methodology. In a 'classical' EELS line scans, the uncertainty 
would be unknown. Despite the increased uncertainty at the interface, the observed trends stay obvious.

Possible improvements for the EELS methodology: A better spatial resolution with a probe corrected STEM. The current literature does not show an environmental probe corrected STEM available. Further, an enhanced recognition of the $\mathrm{Pd} / \mathrm{r}-\mathrm{TiO}_{2}$ interface in the evaluation procedure for every row in the rectangular SI pattern. This can decrease the uncertainty in the immediate vicinity of the interface. Also, other features of the Ti $L$ ELNES can be studied which requires a better spatial resolution and an increased $\mathrm{S} / \mathrm{N}$ ratio, especially in the close vicinity of the interface. Other features can be for example $\mathrm{d} E(b-a), \mathrm{d} E(d-c)$, the intensity ratio $\mathrm{I}\left(\mathrm{L}_{2}\right) / \mathrm{I}\left(\mathrm{L}_{3}\right)$ or even the $\mathrm{O} K$ edge of $\mathrm{r}-\mathrm{TiO}_{2}$ and its fine structure.

Other TEM related techniques can be used to investigate the $\mathrm{Pd} / \mathrm{r}-\mathrm{TiO}_{2}$ near the interface region in the future. Examples are StripeSTEM [135] and smart acquisition EELS [136]. StripeSTEM requires a probe corrected STEM and collects a high-angle annular dark-field image together with a EELS SI pattern. Combining both information StripeSTEM allows for the correlation of spatial and spectroscopic information on an atomic level. Smart acquisition EELS is similar to StripeSTEM, though it is possible to define arbitrary shapes for EELS SI pattern. Therefore, it is not limited to a flat interface. Both techniques have in common that they average over the scan axis that is parallel to the interface. This yields to an enhanced $\mathrm{S} / \mathrm{N}$ ratio of the EEL spectra and allows for reducing the electron dose. However, the disadvantage of both techniques is the loss of the uncertainty for one acquired SI pattern, due to the averaging over the fast scan axis.

\subsection{Consequences for the electronic behavior: mobile species}

Combining the experimental and theoretical findings allows a construction of a final picture of the $\mathrm{Pd} / \mathrm{r}-\mathrm{TiO}_{2}$ system in hydrogen atmosphere which is discussed in the following.

The DFT calculations on the $\mathrm{Pd} / \mathrm{r}-\mathrm{TiO}_{2}$ interface suggest a $\mathrm{H}_{\mathrm{O}}^{0}$ concentration of approximately $3 \cdot 10^{-4}$ per oxygen site in the bulk $\mathrm{r}-\mathrm{TiO}_{2}$ and $\mathrm{a}_{\mathrm{O}}^{+}$concentration of approximately 0.7 per oxygen site at the interface for $\mu_{\mathrm{H}}=-0.14 \mathrm{eV}, \mu_{\mathrm{O}}=-4.35 \mathrm{eV}$ and a Schottky barrier height $\Phi_{\mathrm{B}}^{0}=1 \mathrm{eV}$. The absolute numbers depend on the difference $-\mu_{\mathrm{O}}+\Phi_{\mathrm{B}}^{0}$; both are not exactly known for the produced $\mathrm{Pd} / \mathrm{r}-\mathrm{TiO}_{2}$ system. Hence, the calculations suggest a qualitative picture of the interface: The defect concentration is high at the interface and significantly smaller in the bulk r- $\mathrm{TiO}_{2}$. This suggests the presence of a concentration gradient. In addition, the dominating charge state of $\mathrm{H}_{\mathrm{O}}$ changes from $\mathrm{H}_{\mathrm{O}}^{0}$ in the bulk to $\mathrm{H}_{\mathrm{O}}^{+}$at the interface. Thus, the electronic 
potential $\Phi$ changes and aligns between the $\mathrm{Pd}$ and bulk r- $\mathrm{TiO}_{2}$ site. This is equivalent to the presence of an upwards band bending in the interface vicinity.

The extension of the defect enrichment was estimated via the EELS measurements to be approximately $2 \mathrm{~nm}$. The presence of both band bending and $\mathrm{H}_{\mathrm{O}}$ concentration gradient in the interface vicinity is a fundamental difference to the classical picture of dopants in semiconductors which are fixed in their position [88]. A suggestion for this final situation is sketched in fig. 5.2. The electron chemical potential $\mu_{e}$ aligns between

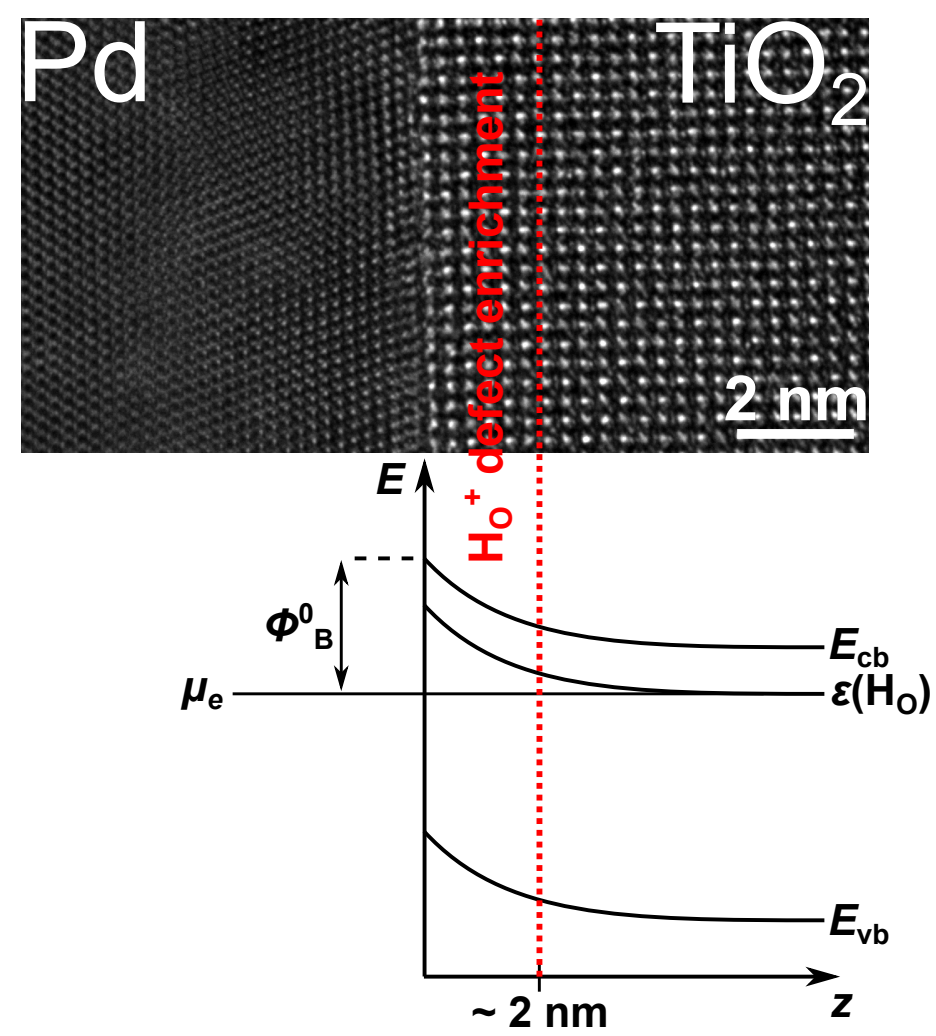

Figure 5.2.: Sketch of the spatial microstructural and electronic situation of the $\mathrm{Pd} / \mathrm{r}-\mathrm{TiO}_{2}$ interface and its vicinity in a hydrogen environment. A high concentration of positively charged $\mathrm{H}_{\mathrm{O}}\left(\mathrm{H}_{\mathrm{O}}^{+}\right)$is located in a $2 \mathrm{~nm}$ vicinity of the interface. The concentration decreases into the bulk $\mathrm{r}-\mathrm{TiO}_{2}$ which exhibits significantly smaller defect concentrations. In bulk $\mathrm{r}-\mathrm{TiO}_{2}$ the neutrally charged $\mathrm{H}_{\mathrm{O}}\left(\mathrm{H}_{\mathrm{O}}^{0}\right)$ is the dominant defect. The alignment of the electron chemical potential $\mu_{\mathrm{e}}$ is accompanied by a upwards band bending in the interface vicinity. The appearance of high defect concentrations require a relatively high mobility of the defects. In collaboration with M. Sotoudeh, M. Wenderoth, P. Blöchl and A. Pundt.

the $\mathrm{Pd}$ and $\mathrm{r}-\mathrm{TiO}_{2}$ side. The $\mathrm{r}-\mathrm{TiO}_{2}$ side is defined by the charge state level $\epsilon\left(\mathrm{H}_{\mathrm{O}}\right)$ of $\mathrm{r}-\mathrm{TiO}_{2}$ being dominated by $\mathrm{H}_{\mathrm{O}}$. The result is an upwards band bending building up in the $\mathrm{r}-\mathrm{TiO}_{2}$ in the close vicinity of the $\mathrm{Pd} / \mathrm{r}-\mathrm{TiO}_{2}$ interface. 
It is suggested that the majority of the interface is represented by this situation which could be an explanation for the homogeneous lowering of the Schottky barrier height described by Cerchez et al. [12]. The 'small' extension of the 'defective' layer is in line with the experiments from Yamamoto et al. [10], Kobayashi et al. [11] and Cerchez et al. [12]. The maximum effect is expected to be directly at the interface [1013], though the interface (defined as the bonding plane between $\mathrm{Pd}$ and $\mathrm{r}-\mathrm{TiO}_{2}$ ) itself is not accessible by the presented EELS technique.

Hydrogen has a concentration of approximately $500 \mathrm{ppb}$ in the environmental air [66, 137]. This corresponds to a partial pressure of $5 \cdot 10^{-2} \mathrm{~Pa}$. The calculations of the thermodynamic of the $\mathrm{Pd} / \mathrm{r}-\mathrm{TiO}_{2}$ system suggested a significant defect concentration already at these conditions for reduced $n$-type r- $\mathrm{TiO}_{2}$. Thus, $\mathrm{Pd} / \mathrm{r}-\mathrm{TiO}_{2}$ systems used in normal air can be (strongly) affected by hydrogen.

The appearance of high defect concentrations requires a high defect mobility in timescales $(\sim 1 \mathrm{~h})$ that were used in the experiment. One possibility is the transport of $\mathrm{H}_{\mathrm{O}}$ defects from the bulk $\mathrm{r}-\mathrm{TiO}_{2}$ into the interface vicininty. Another possibility is oxygen loss due to the formation of oxygen or water molecules, cf. chap. 2.1.3 and 2.1.4. Based on the presented experiments and calculations, it is not possible to judge which process may dominate. 



\section{Summary}

In order to get insights into the hydrogen interaction with the $\mathrm{Pd} / \mathrm{r}-\mathrm{TiO}_{2}$ system, in situ measurements were carried out in different low pressure hydrogen gas environments in an environmental transmission electron microscope (ETEM): Changes in the local chemistry of $\mathrm{r}-\mathrm{TiO}_{2}$ were investigated by electron energy loss spectroscopy (EELS) utilizing monochromated scanning transmission electron microscopy (STEM). High resolution STEM (HR-STEM) investigations were carried out to observe changes in the dislocations density $\left(\rho_{\mathrm{D}}\right)$ of $\mathrm{Pd}$. The ETEM measurements were supported by density functional theory (DFT) calculations and variable energy positron annihilation spectroscopy (VEPAS). VEPAS allows the investigation of concentration changes of vacancies $\left(\mathrm{V}_{\mathrm{Pd}}\right)$ and dislocations $\left(\perp_{\mathrm{Pd}}\right)$ in $\mathrm{Pd}$ and of titanium vacancies $\left(\mathrm{V}_{\mathrm{Ti}}\right)$ in $\mathrm{r}$ $\mathrm{TiO}_{2}$. VEPAS was applied on prepared $\mathrm{Pd} / \mathrm{r}-\mathrm{TiO}_{2}$ samples in the prepared (or virgin) state and on the same samples at different hydrogen gas pressures. The DFT calculations were carried out by $\mathrm{M}$. Sotoudeh on pure $\mathrm{r}-\mathrm{TiO}_{2}$ and on $\mathrm{r}-\mathrm{TiO}_{2}$ containing oxygen vacancies $\left(\mathrm{V}_{\mathrm{O}}\right)$, interstitial hydrogen $\left(\mathrm{H}_{\mathrm{i}}\right)$ and substitutional hydrogen $\left(\mathrm{H}_{\mathrm{O}}\right)$. In a joint cooperation, the defect influences on the density of states (DoS) of $\mathrm{r}-\mathrm{TiO}_{2}$ were investigated. This finally allowed an interpretation of the acquired EELS data based on these defects in $\mathrm{r}-\mathrm{TiO}_{2}$ and yielded a final picture of the $\mathrm{Pd} / \mathrm{r}-\mathrm{TiO}_{2}$ system in a hydrogen environment.

The $\mathrm{Pd} / \mathrm{r}-\mathrm{TiO}_{2}$ samples were optimized for large grain sizes and interface sharpness. Subsequently, the samples were characterized via AFM, XPS, XRD, pole figure, $\phi$-scan and HR-TEM investigations. The pretreated $\mathrm{r}-\mathrm{TiO}_{2}(110)$ crystals exhibited a clean surface with sharp terraces and steps. The Pd films were deposited at $1023 \mathrm{~K}$ and grew with two preferred orientations on the $\mathrm{r}-\mathrm{TiO}_{2}(110)$ crystals. Both orientations exhibited the same orientation relationship, namely $[\overline{2} 11](111) \|[\overline{1} 10](110) \mathrm{r}-\mathrm{TiO}_{2}$ which is depicted in fig. 6.1. The $\mathrm{Pd} / \mathrm{r}-\mathrm{TiO}_{2}$ samples exhibited minimal microstructural contributions and a semi-coherent interface which was found to be one to two monolayers sharp. A comparison with literature data predicted the $\mathrm{r}-\mathrm{TiO}_{2}$ crystals to be of $n$-type conductivity with $\mathrm{V}_{\mathrm{O}}$ being the dominant intrinsic defect. $I$ - $V$ measurements on a $\mathrm{Pd} / \mathrm{r}-\mathrm{TiO}_{2}$ sample showed a Schottky behavior and hydrogen gas sensing capability.

VEPAS was carried out on the optimized $\mathrm{Pd} / \mathrm{r}-\mathrm{TiO}_{2}$ samples in the prepared state and in hydrogen environments. Hydrogen pressures ranging from $1 \mathrm{~Pa}$ to $1000 \mathrm{~Pa}$ were applied. The VEPAS measurements revealed no hydrogen induced changes in the interface vicinity or in the bulk of the $\mathrm{r}-\mathrm{TiO}_{2}$ in this pressure range. This finding indicates that no change appears in the $\mathrm{V}_{\mathrm{Ti}}$ concentration. However, hydrogen induced 


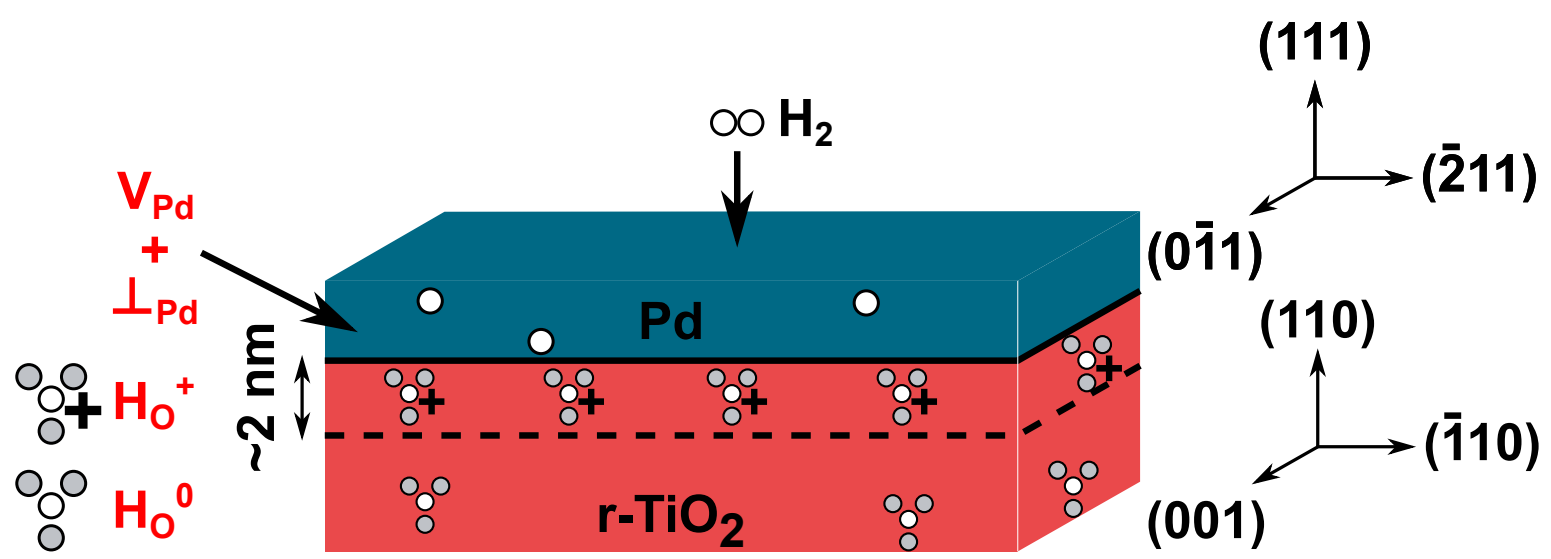

Figure 6.1.: Concluding picture of the $\mathrm{Pd} / \mathrm{r}-\mathrm{TiO}_{2}$ system in a low pressure hydrogen gas environment. Indicated with the solid black line is the $\mathrm{Pd} / \mathrm{rutile} \mathrm{TiO}_{2}$ interface. The observed orientation relationship of $\mathrm{Pd}$ on the $\mathrm{r}-\mathrm{TiO}_{2}$ crystals is given on the right hand side. In the $\mathrm{Pd}$ film vacancies $\mathrm{V}_{\mathrm{Pd}}$ and dislocations $\perp_{\mathrm{Pd}}$ appear. Additionally, on the oxygen reduced $\mathrm{r}-\mathrm{TiO}_{2}$ side neutrally charged substitutional hydrogen $\mathrm{H}_{\mathrm{O}}^{0}$ is the dominant defect far from the interface. The $2 \mathrm{~nm}$ vicinity of the $\mathrm{Pd} / \mathrm{r}-\mathrm{TiO}_{2}$ interface is indicted with the dashed black line. Here, positively charged substitutional hydrogen $\mathrm{H}_{\mathrm{O}}^{+}$dominates, with significantly higher concentrations as compared to the bulk. Coloring after CPK coloring of 'Jmol' [14].

changes were found in the $\mathrm{Pd}$ film. The average positron diffusion length $\mathrm{L}_{+}$was found to decrease in the hydrogen environment. Open volume defects in $\mathrm{Pd}$ as $\mathrm{V}_{\mathrm{Pd}}$ and $\perp_{\mathrm{Pd}}$ can explain this finding, cf. Pd part of fig. 6.1. But with VEPAS, these defects cannot be distinguished. Therefore, HR-STEM was applied during in situ hydrogen loading of a $\mathrm{Pd} / \mathrm{r}-\mathrm{TiO}_{2}$ sample in the ETEM. HR-STEM images were obtained for the initial state and for different hydrogen pressures in the middle of the Pd film. From these images, the dislocation area density $\rho_{\mathrm{D}}^{(111)}$ in the $\operatorname{Pd}(111)$ planes was evaluated as a function of the hydrogen pressure. No significant changes in $\rho_{\mathrm{D}}^{(111)}$ were observed for hydrogen pressures ranging from $0.1 \mathrm{~Pa}$ to $100 \mathrm{~Pa}$. Only at $500 \mathrm{~Pa}$ hydrogen did $\rho_{\mathrm{D}}^{(111)}$ increase about one order of magnitude. This finding suggests that new open volume from $\perp_{\mathrm{Pd}}$ can only be created at hydrogen pressures above $100 \mathrm{~Pa}$. Hence, the observed decrease of $\mathrm{L}_{+}$for pressures of $100 \mathrm{~Pa}$ and below is explained with the creation of $\mathrm{V}_{\mathrm{Pd}}$.

An approach was developed to study the interface vicinity locally with EELS in situ in an ETEM. High requirements were put on the EELS methodology as well as on the samples. To reach these requirements an EELS methodology was developed including an optimized set of parameters and a reliable and automated evaluation of the acquired EEL spectra. With the developed EELS methodology and the optimized samples, the local chemistry was studied on the titanium (Ti) $L$ edge with EELS and small changes in the Ti $L$ edge's fine structure were investigated as a function of the hydrogen pressure and the interface distance. 
The EELS methodology was successfully established and allowed the detection of energetic changes in the Ti $L$ ELNES of approximately $100 \mathrm{meV}$ with a spatial resolution of approximately $1 \mathrm{~nm}$. Local in situ EELS measurements of the Ti $L$ ELNES were performed in the ETEM in hydrogen gas atmosphere at different pressures. Up to a pressure of $1 \mathrm{~Pa}$, the EELS measurements revealed no changes in the Ti $L$ ELNES; neither in the bulk nor in the interface vicinity. At a hydrogen pressure of $10 \mathrm{~Pa}$, however, changes were found in the interface vicinity, but not in the bulk. These changes in the Ti $L$ ELNES were evaluated by an investigation of the energy splitting $\mathrm{d} E\left(b^{\prime}-a\right)$, of difference spectra and of the center of gravity (CoG) analysis of the $b / b^{\prime}$ white line and the $e_{g}$ states, respectively. The energy splitting $\mathrm{d} E\left(b^{\prime}-a\right)$ decreases approximately $150 \mathrm{meV}$. A similar decrease was observed at different places of the $\mathrm{Pd} / \mathrm{r}-\mathrm{TiO}_{2}$, which suggests homogeneous behavior throughout the whole interface vicinity. By EELS difference spectra, the decreases of $\mathrm{d} E\left(b^{\prime}-a\right)$ were reinterpreted as a shift of states in the underlying DoS on the order of $1 \mathrm{eV}$. The CoG shift was quantified from EELS spectra as approximately $-170 \mathrm{meV}$ approximately $0.5 \mathrm{~nm}$ away from the interface. The extension of the observed changes was found to be present within $2 \mathrm{~nm}$ of the interface. Hence, the in situ EELS investigations suggest a shift of the $e_{g}$-like states in underlying $\mathrm{DoS}$ of $\mathrm{r}-\mathrm{TiO}_{2}$ at $10 \mathrm{~Pa}$ hydrogen.

The VEPAS studies exclude the presence of Ti related defects in $\mathrm{r}-\mathrm{TiO}_{2}$. Therefore only oxygen and hydrogen related defects can be considered to explain the changes observed in the EELS experiments.

DFT calculations were carried out by M. Sotoudeh and P. Blöchl for the three important defects in $\mathrm{r}-\mathrm{TiO}_{2}$ : namely $\mathrm{V}_{\mathrm{O}}, \mathrm{H}_{\mathrm{i}}$ and $\mathrm{H}_{\mathrm{O}}$. The last defect replaces one oxygen atom in the $\mathrm{r}-\mathrm{TiO}_{2}$ lattice with one hydrogen atom. The DFT calculations investigated the thermodynamics of these defects and a $\mathrm{Pd} / \mathrm{r}-\mathrm{TiO}_{2}$ interface was simulated in an electrostatic picture. These calculations suggested that substitutional hydrogen is the dominant defect in $\mathrm{r}-\mathrm{TiO}_{2}$ for the experimental ranges of oxygen and hydrogen chemical potential (or pressure). The substitutional hydrogen was found to be present in its positive charge state $\mathrm{H}_{\mathrm{O}}^{+}$at the interface and its vicinity in substantially higher concentrations than in the bulk, cf. fig. 6.1. Therefore, a concentration gradient of $\mathrm{H}_{\mathrm{O}}$ is present from the interface to the bulk $\mathrm{r}-\mathrm{TiO}_{2}$. It is concluded that to keep the electron chemical potential spatially constant over the whole contact the gradient is accompanied by band bending, cf. fig. 5.2. The concentration of substitutional hydrogen was shown to depend strongly on the hydrogen chemical potential. Therefore, a strong hydrogen pressure dependency could be expected. This is in line with the pressure dependency found during the in situ EELS experiments. The DoS of substitutional hydrogen in $\mathrm{r}-\mathrm{TiO}_{2}$ revealed shifted states between the $t_{2 g^{-}}$and $e_{g}$-like states. This chemical shift is similar to the one observed by EELS. Therefore it was concluded that the presence of $\mathrm{H}_{\mathrm{O}}^{+}$is responsible for the observed changes in the EEL spectra within $2 \mathrm{~nm}$ of the $\mathrm{Pd} / \mathrm{r}-\mathrm{TiO}_{2}$ interface, cf. fig. 6.1. Based on the DFT calculations, the relative $\mathrm{CoG}$ shift was calculated to be comparable to the one obtained by EELS. This comparison revealed a high concentration of positively charged substitutional 
hydrogen in the vicinity of the interface. A rough estimate yielded a concentration on the order of $10 \%$. But, the concentration estimate turned out to be unstable against shifts of the used integration windows.

This work shows examples of hydrogen interacting with the $\mathrm{Pd} / \mathrm{r}-\mathrm{TiO}_{2}$ model system. Hydrogen incorporated in the $\mathrm{r}-\mathrm{TiO}_{2}$, and especially in the close vicinity of the $\mathrm{Pd} / \mathrm{r}-\mathrm{TiO}_{2}$ interface, can result in changed behavior of the system, allowing gas sensing and other related applications. Likewise, in catalytic applications, defect states are believed to be the catalytic centers, as, for example, on Pd surfaces. Hence, the environment changing the samples' defect chemistry and defect concentration significantly can in turn influence the activity of the system. This highlights the importance of fundamental studies, like this one, of interfaces and surfaces and their interaction with the environment (reactants) in catalytic processes. 


\section{A. Appendix}

\section{A.1. Addition to theory}
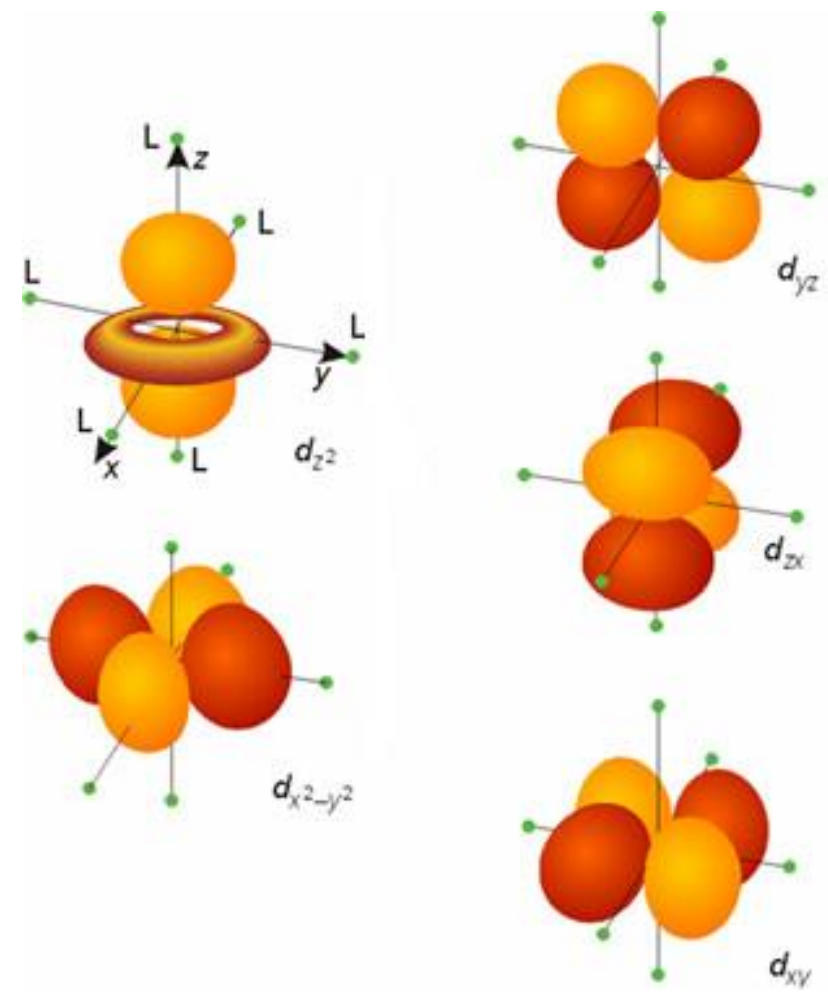

Figure A.1.: The five $3 d$ orbitals are shown with respect to a $x$-, $y$ - and $z$-axis. Assuming a Ti atom in the zero of the three axis and the green dots to be the oxygen atoms of an $\mathrm{TiO}_{6}$ octahedron allows understanding the contribution of the individual orbitals to $t_{2 g}$ and $e_{g}$ states. The $d_{y z}, d_{z x}$ and $d_{x y}$ orbitals show in between the axes. Therefore, they exhibit a $t_{2 g}$ nature. The $d_{z^{2}}$ and $d_{x^{2}-y^{2}}$ orbitals lie on the axes and, hence, exhibit $e_{g}$ nature. Image provided by J3D3 [138] under the CreativeCommons BY-SA 4.0 [139] license. 


\section{A.2. Thin Pd film prepared at room temperature (RT)}

Thin Pd films can exhibit high lateral stresses on suitable substrates as rutile (cf. chap. 2.3.1). The thin Pd films were deposited at RT. The mobility of the Pd atoms on the surface, therefore, was limited and dewetting was not influencing the film growth. The thin Pd films were investigated in the view of their thickness (XRR), surface flatness (AFM, XRR) and crystal orientation (XRD).

X-ray reflectometry was applied on a thin $\mathrm{Pd}$ film. The corresponding measurement is shown in fig. A.2. The reflected $\mathrm{x}$-ray intensity is presented here against $2 \Theta$. The

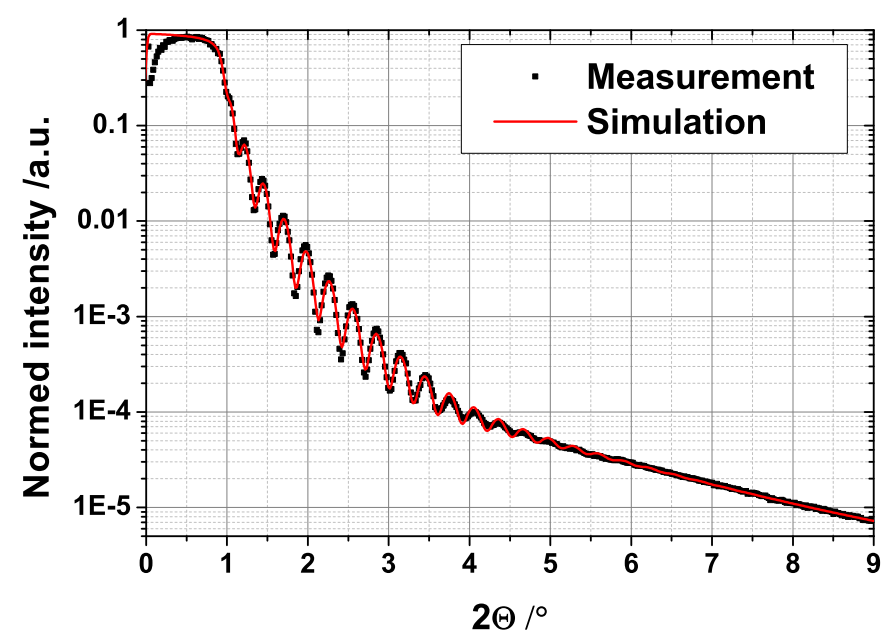

Figure A.2.: Measured reflectometry diagram of a thin Pd film. Shown is the normed intensity as a function of $2 \Theta$. The simulation (red line) fits well to the measured data (black squares). The simulation gives $27.96 \mathrm{~nm}$ total thickness and $0.20 \mathrm{~nm}$ surface roughness.

simulation performed with the LEPTOS software described the measurement appropriately, the final parameters are presented in tab. A.1. The total thickness of the Pd

\begin{tabular}{lccc}
\hline \hline material & thickness /nm & roughness $/ \mathrm{nm}$ & density \\
\hline $\mathrm{PdO}$ & 1.0387 & 0.1993 & 8.30 \\
$\mathrm{Pd}$ & 26.9177 & 0.5154 & 12.01020 \\
$\mathrm{TiO}_{2}$ & - & 0.6819 & 4.2490 \\
\hline \hline
\end{tabular}

Table A.1.: Resulting parameters of the XRR simulation via the LEPTOS software of the measurements shown in fig. A.2. The density was kept fixed for the simulation, therefore only the thickness and the roughness of the layers were fitted.

film was $28.0 \mathrm{~nm}$ and revealed a roughness of $0.2 \mathrm{~nm}$. The thin Pd films were there- 
fore slightly rougher than the rutile substrate surfaces that exhibited a roughness of $0.1 \mathrm{~nm}^{1}$. A palladium oxide $(\mathrm{PdO})$ layer was found on all, with XRR investigated, thin $\mathrm{Pd}$ film. Interestingly, this passivation layer was always in the range of $1 \mathrm{~nm}$ to $2 \mathrm{~nm}$ which seems to be its thickness under atmospheric conditions. That a surface oxide forms was not surprising and is predicted by the corresponding Ellingham diagram of $\mathrm{PdO}[140]$.

AFM was used to additionally investigate the surface of the $28.0 \mathrm{~nm} \mathrm{Pd} \mathrm{film.} \mathrm{The}$ corresponding height profile is shown in fig. A.3. The root mean square roughness was

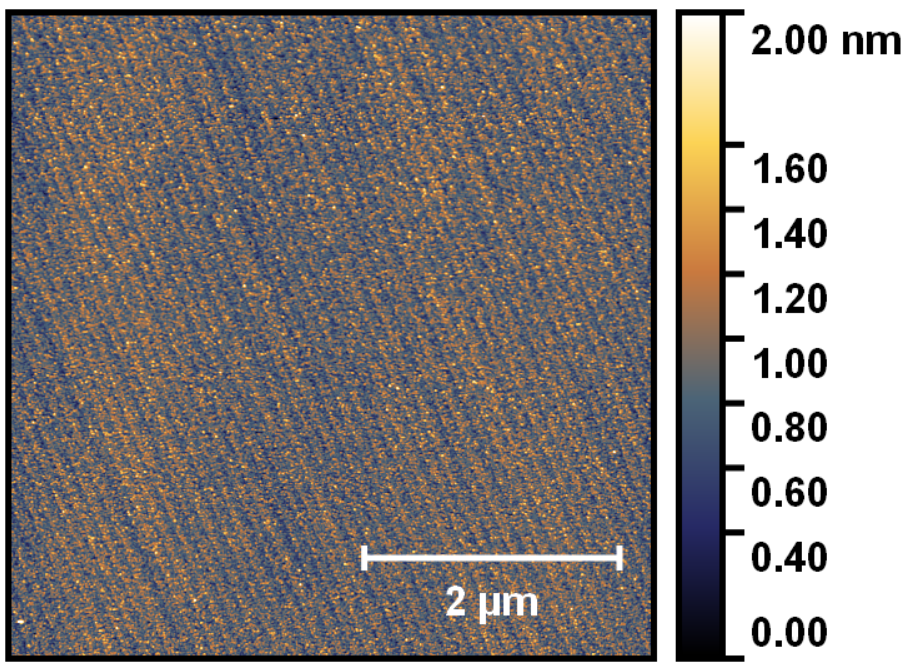

Figure A.3.: $5 \times 5 \mathrm{\mu m}^{2}$ AFM height profile of a $28 \mathrm{~nm}$ Pd film. The terrace structure of the substrate is visible. The root mean square roughness is approximately $0.2 \mathrm{~nm}$. A grain size in the order of $10 \mathrm{~nm}$ can be estimated.

approximately $0.2 \mathrm{~nm}$ which is in total agreement with the XRR measurement above. A rough estimate of the grain size was done, assuming the visible height oscillations to be single grains which results in a size in the order of $10 \mathrm{~nm}$. The original terrace structure of the rutile substrate (cf. fig. 4.1b) was maintained for the thin Pd film deposited at RT. Therefore, the Pd film has adopted the surface structure of the rutile substrates.

X-ray diffraction was applied on the $28 \mathrm{~nm} \mathrm{Pd}$ film and the corresponding measurement is shown in fig. A.4. Three interferences were observed originating from $\operatorname{Pd}(111)$ and rutile(220). No other Pd orientations were observed. The maximum of the $\operatorname{Pd}(111)$ interference is observed at $2 \Theta=(40.109 \pm 0.006)^{\circ}$. This results in

\footnotetext{
${ }^{1}$ In collaboration with Niklas Herwig [93] via AFM and XRR measurements.
} 


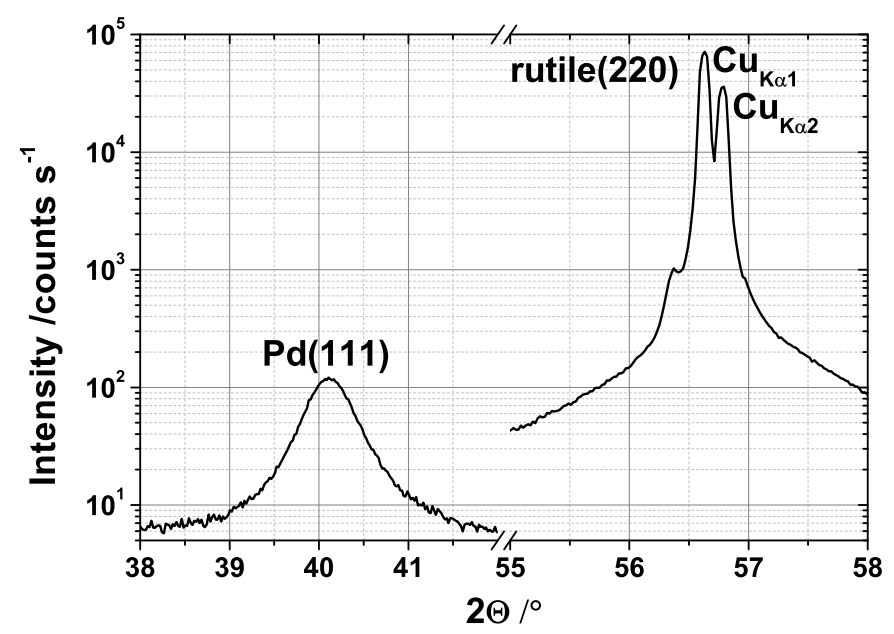

Figure A.4.: XR diffractogram showing the measured intensity as a function of $2 \Theta$ of a $28 \mathrm{~nm} \mathrm{Pd}$ film. The deposition was performed at RT, the other conditions are summarized in tab. 4.1. The selected angle range shows the $\operatorname{Pd}(111)$ interference as well as the rutile(220) interferences for the $\mathrm{Cu} K_{\alpha_{1}}$ and $K_{\alpha_{2}}$ line.

$d_{111}=(2.2463 \pm 0.0006) \AA$, which is in good agreement with the value from Arblaster [72] with $(2.2460 \pm 0.0004) \AA$. Thus, the $28 \mathrm{~nm} \mathrm{Pd}$ film did not reveal a significant expansion or compression in normal direction. Hence, the lateral stresses are comparable small or compensated each other for this film thickness.

As a comparison a $16.4 \mathrm{~nm} \mathrm{Pd}$ film ${ }^{2}$ - prepared under the same conditions as the $28 \mathrm{~nm}$ Pd film - was measured with XRD. The corresponding XR diffractogram is shown in fig. A.5. Again three interferences were observed originating from $\operatorname{Pd}(111)$ and rutile(220) and no other Pd orientations were observed. The Laue oscillations of the $\operatorname{Pd}(111)$ interference qualitatively proofed a high flatness of the interface and surface of the $\mathrm{Pd}$ film. The maximum of the $\mathrm{Pd}(111)$ interference was observed at $2 \Theta=(40.2372 \pm 0.0022)^{\circ}$. This results in $d_{111}=(2.2395 \pm 0.0003) \AA$. As for the $200 \mathrm{~nm}$ $\mathrm{Pd}$ film, cf. chap. 4.1.2.1), the $16.4 \mathrm{~nm} \mathrm{Pd}$ film is compressed in normal direction. The Pd lattice compression in normal direction was $\Delta d_{111}=-(0.0065 \pm 0.0005) \AA$ when compared to the bulk value from Arblaster [72]. This corresponded to a strain $\epsilon_{111}=-(0.0029 \pm 0.0002)$ in normal direction.

\footnotetext{
${ }^{2}$ The corresponding XRR measurement is not shown.
} 


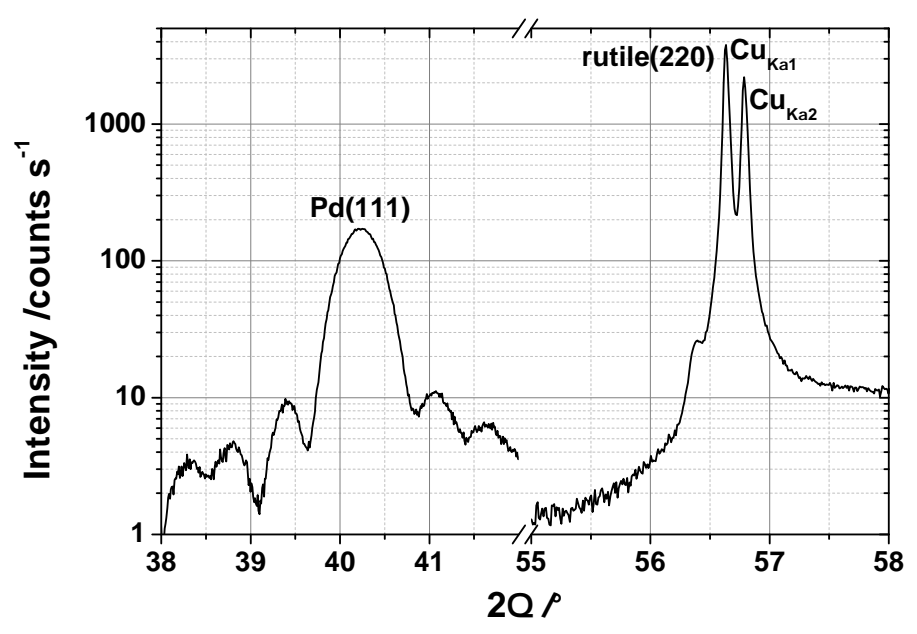

Figure A.5.: XR diffractogram showing the measured intensity as a function of $2 \Theta$ for a $16.4 \mathrm{~nm} \mathrm{Pd}$ film prepared at RT. The deposition conditions are summarized in tab. 4.1. The selected angle range shows the $\operatorname{Pd}(111)$ interference as well as the rutile(220) interferences for the $\mathrm{Cu} K_{\alpha_{1}}$ and $K_{\alpha_{2}}$ line. Laue oscillations are visible on the left and right side of the $\operatorname{Pd}(111)$ interference.

In summary, the thin $\mathrm{Pd}$ films deposited at RT revealed a nanocrystalline structure. The films grew with (111) orientation on the rutile(110) substrate. Their surface were relatively flat. The $28 \mathrm{~nm} \mathrm{Pd}$ film did not show significant compression in normal direction while the $16.4 \mathrm{~nm} \mathrm{Pd}$ film revealed a compression of $0.3 \%$. This is in line with former studies of Wagner et al. [141]. They reported a thickness dependency for the lateral stress state for Pd on sapphire for thicknesses up to $200 \mathrm{~nm}$. 


\section{A.3. X-ray investigations}

\section{A.3.1. Pole figure measurements and $\phi$ scans}

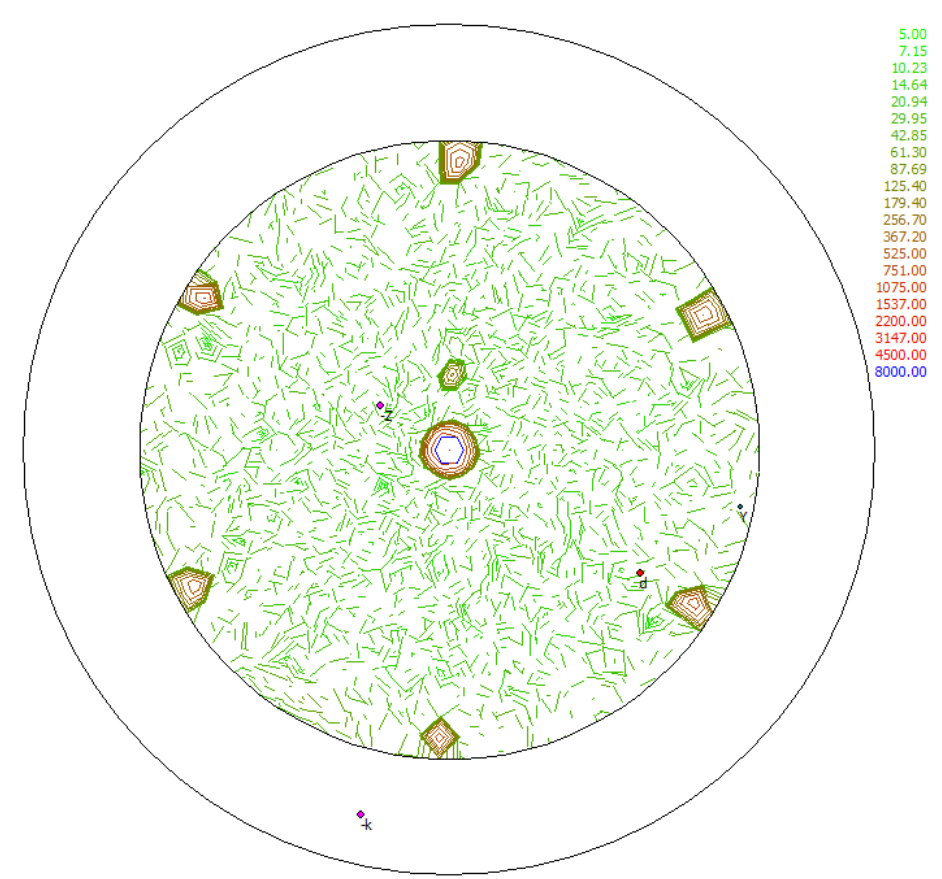

Figure A.6.: $\mathrm{Pd}(111)$ pole figure of the $200 \mathrm{~nm} \mathrm{Pd}$ film deposited at $1023.15 \mathrm{~K}$ displayed on an exponential scale. A weak third orientation is visible, which is tilted out from the center by approximately $20^{\circ}$.

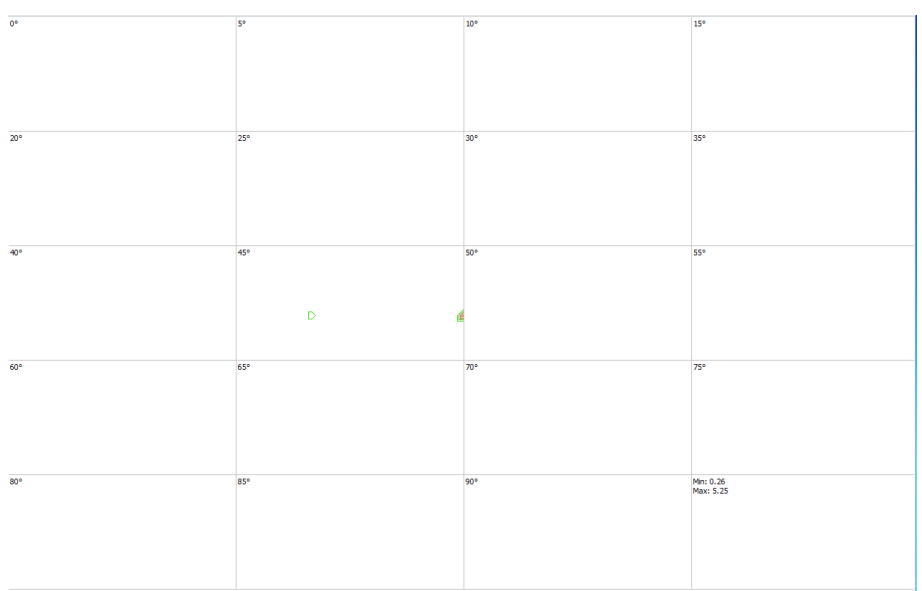

Figure A.7.: The orientation distribution function (ODF) for the $200 \mathrm{~nm} \mathrm{Pd}$ film deposited at $1023.15 \mathrm{~K}$. 


\section{A.3.2. X-ray reflectometry}

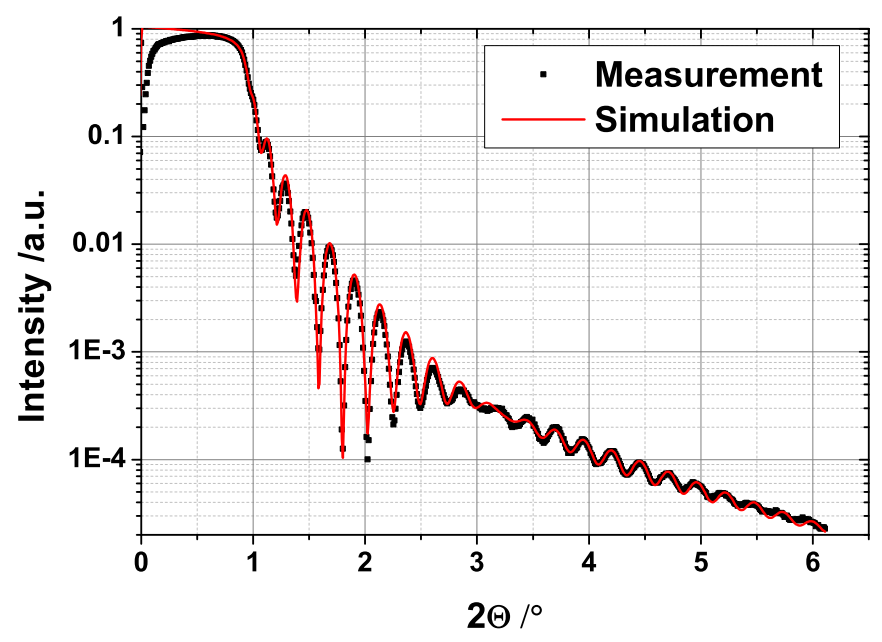

Figure A.8.: Measured reflectometry diagram of an $37 \mathrm{~nm}$ Pd film deposited at RT. Shown is the normed intensity as a function of $2 \Theta$. The simulation (red line) fits well to the measured data (black squares). The simulation gives $34.93 \mathrm{~nm}$ total thickness and $0.78 \mathrm{~nm}$ surface roughness. All simulation parameters are summarized in tab. A.2.

\begin{tabular}{lccc}
\hline \hline material & thickness /nm & roughness /nm & density \\
\hline $\mathrm{PdO}$ & 1.403 & 0.7849 & 8.30 \\
$\mathrm{Pd}$ & 35.5300 & 0.5081 & 12.01020 \\
$\mathrm{TiO}_{2}$ & - & 0.1006 & 0.0001 \\
\hline \hline
\end{tabular}

Table A.2.: Resulting parameters of the XRR simulation shown in fig. A.8. The density is kept fixed for the simulations, therefore just the thickness and the roughness of the layers is fitted. Due to the strong absorption of the Pd the substrate is no more visible and therefore the density needed to be set to zero.

\section{A.4. Constraints for the lateral stress calculation}

In the following it is shown that the three constraints, given in chap. 3.5.1.2, are satisfied for a $\operatorname{Pd}(111)$ film.

The deposited $\mathrm{Pd}$ films grow in (111) orientation on the $\mathrm{r}-\mathrm{TiO}_{2}(110)$ substrates, no other orientation were present in normal direction as confirmed with XRD in chap. 4.1.2. All grains therefore contribute to the measured $\operatorname{Pd}(111)$ interference and the first constraint is fulfilled. The penetration depth $\tau$ for $\mathrm{Cu} K_{\alpha}$ radiation $(\sim 8 \mathrm{keV}[76])$ in $\mathrm{Pd}$ is $1.23 \mu \mathrm{m}$ [77]. With $\tau$ the intensity $I\left(z^{\prime}\right)$ can be calculated, 
which is scattered in a depth $z^{\prime}$ and remains from the initial intensity $I_{0} . I\left(z^{\prime}\right) / I_{0}$ as a function of $z^{\prime}$ is shown for Pd in fig. A.9. In a depth of $200 \mathrm{~nm}$ (which is about the

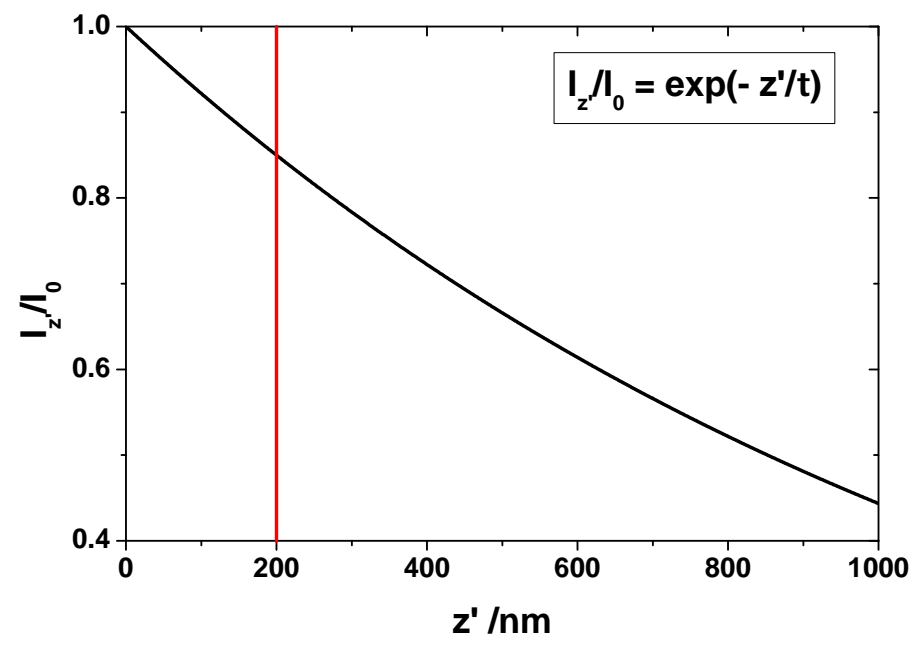

Figure A.9.: Shown is the amount of intensity $I\left(z^{\prime}\right)$ that leaves the sample surface after scattering in the depth $z^{\prime}$. For simplicity $I\left(z^{\prime}\right)$ is normed to the intensity of the initial beam $I_{0}$. In this work the maximum Pd layer thickness is approximately $200 \mathrm{~nm}$ (red line). Up to that thickness, every depth contributes nearly equal. Therefore, no depth dependency is expected.

thickness of the thicker Pd films) an intensity of $85 \%$ of $I_{0}$ can be measured. Thus, for the Pd film studied in this work the measurement averages over the whole film thickness. No thickness dependency is expected. Thus, the second constraint is fulfilled as well. $\mathrm{Pd}$ is isotropic in its elastic constants orthogonal to the (111) direction as calculated for example by Wagner [81, chap. 2.3.3]. The third constraint is therefore fulfilled as well.

\section{A.5. Alignment of the TEM and EELS acquisition parameters}

The FEI Titan ETEM is a complex microscope that includes many components that prone to drift. For each component this drift might be small but collected from the gun up to the GIF it can influence the EELS experiments significantly, details on this are given in chap. 3.3.1. Therefore all drift had to be minimized beforehand to conduct a successful experiment. The ETEM was set up and aligned in the following way:

- The TEM lamella was transferred in the microscope and aligned.

- In TEM mode a full tune of the GIF was performed. 
- The microscope was set to STEM mode and aligned.

- The monochromator was excited and the STEM mode was aligned again.

- The freshly excited monochromator typically exhibited quiet high drift. It had therefore to equilibrate, here this was done over night ${ }^{3}(\sim 12 \mathrm{~h})$.

- Finally the STEM mode was aligned once again with the exited and stabilized monochromator.

In the following the acquisition parameters are summarized that yielded to EEL spectra best suited (cf. chap. 3.3.1) for the in situ experiments:

- TEM acceleration voltage: $300 \mathrm{kV}$

- Monochromator excitation: 1.0

- Monochromator mono spot size: 15

- Spot: 11

- Magnification: $910 \mathrm{kx}$

- Camera length ${ }^{4}: 38 \mathrm{~mm}$

- Screen current: $~ 130 \mathrm{pA}$

- Spatial drift correction: used after each fourth row

- Convergence semi-angle: $\alpha=22.2 \mathrm{mrad}$

- Collection semi-angle: $\beta=21.9 \mathrm{mrad}$

- GIF entrance aperture: $2.5 \mathrm{~mm}$

- Spectrometer dispersion: $0.05 \mathrm{eV} / \mathrm{ch}$ or $0.1 \mathrm{eV} / \mathrm{ch}$

- Detector binning: $130 \mathrm{x}$

- Dual EELS: the low loss window started at an energy loss of $-10 \mathrm{eV}$ and the high loss window started at $390 \mathrm{eV}$.

- Dark current correction: Used after each SI pattern

\footnotetext{
${ }^{3}$ From experience at least $6 \mathrm{~h}$ were needed until the monochromator stabilized and the main drift had vanished.

${ }^{4}$ The GIF entrance aperture plane changes as a function of the projector (system) excitation. In STEM mode the crossover can be changed by the camera length therefore this parameter is fixed for all experiments.
} 
- SI pattern step sizes: $0.5 \mathrm{~nm}$ or $1 \mathrm{~nm}$ (better results were obtained with the first one)

- SI pattern size: $20 \mathrm{~nm}$ (y-direction) $\times 25 \mathrm{~nm}$ (z-direction), cf. fig. 3.14

- Integration time per pixel in low loss: $\sim 0.2 \mathrm{~ms}$

- Integration time per pixel in core loss: $0.01 \mathrm{~s}$ to $0.05 \mathrm{~s}$

- Total collection time of SI pattern: 1 min to 3 min

\section{A.6. Local EELS on hydrogen loaded Pd/rutile}

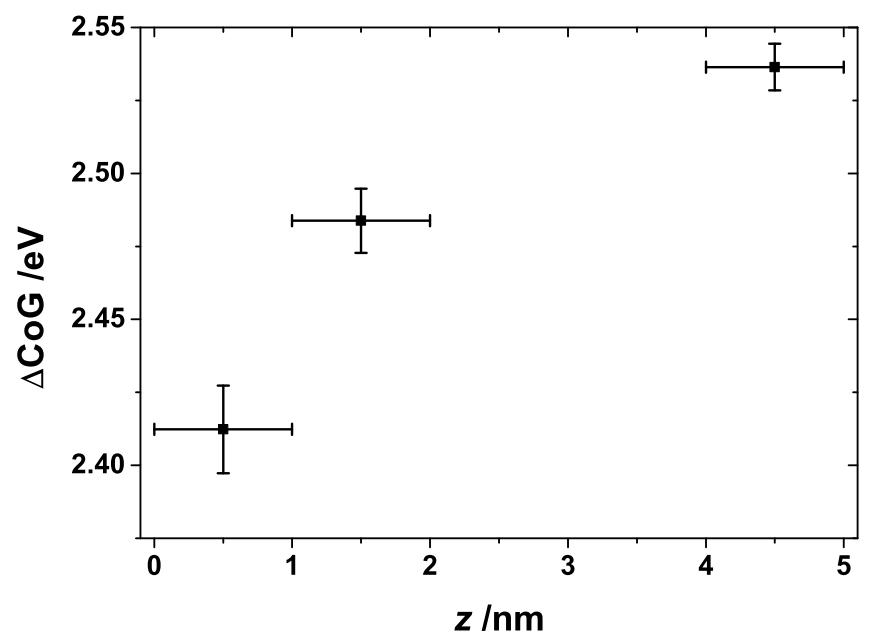

Figure A.10.: $\triangle \mathrm{CoG}$, see eq. 3.2, is shown as a function of $z$. The $4.5 \mathrm{~nm}$ spectrum from fig. 4.12a was taken as the reference since it did not show hydrogen related changes. In the close vicinity of the $\mathrm{Pd} /$ rutile interface $(0.5 \mathrm{~nm}$ and $1.5 \mathrm{~nm}$ spectrum) and in a hydrogen pressure of $10 \mathrm{~Pa}, \Delta \mathrm{CoG}$ got smaller. Therefore, the energy difference between the $\mathrm{CoG}\left(b / b^{\prime}\right)$ and $\mathrm{CoG}(a)$ got smaller in $\mathrm{r}-\mathrm{TiO}_{2}$ in the $\mathrm{Pd} / \mathrm{r}-\mathrm{TiO}_{2}$ interface vicininty.

\section{A.7. Formulas}

The weighted average $\bar{x}$ was calculated using the individual values $x_{i}$ and their corresponding uncertainties $\sigma_{i}$ as follows:

$$
\bar{x}=\frac{\sum_{i} \frac{x_{i}}{\sigma_{i}^{2}}}{\sum_{i} \frac{1}{\sigma_{i}^{2}}}
$$


The corresponding uncertainty of the weighted average $\bar{\sigma}$ was calculated via:

$$
\bar{\sigma}=\sqrt{\frac{1}{\sum_{i} \frac{1}{\sigma_{i}^{2}}}}
$$





\section{Bibliography}

[1] J. Nowotny, C. C. Sorrell, T. Bak and L. R. Sheppard. Solar-hydrogen: Unresolved problems in solid-state science. Solar Energy (2005) 78(5) 593602. doi:10.1016/j.solener.2005.01.008. URL http://www.sciencedirect.com/ science/article/pii/S0038092X05000551.

[2] J. Winkler. Titandioxid. Vincentz Network, 1. edition (2003). ISBN 9783878707387.

[3] M. K. Nowotny, L. R. Sheppard, T. Bak and J. Nowotny. Defect Chemistry of Titanium Dioxide. Application of Defect Engineering in Processing of $\mathrm{TiO}_{2}$ Based Photocatalysts. The Journal of Physical Chemistry C (2008) 112(14) 52755300. doi:10.1021/jp077275m. URL http://pubs .acs .org/doi/abs/10.1021/ jp077275m.

[4] G. J. Hill. The effect of hydrogen on the electrical properties of rutile. Journal of Physics D: Applied Physics (1968) 1(9) 1151-1162. doi:10.1088/0022-3727/1/9/ 308. URL http://iopscience.iop.org/article/10.1088/0022-3727/1/9/ 308.

[5] K. Hashimoto, H. Irie and A. Fujishima. TiO $\mathrm{O}_{2}$ Photocatalysis: A Historical Overview and Future Prospects. Japanese journal of applied physics (2005) 44(12) 8269-8285. doi:10.1143/JJAP.44.8269. URL http:// iopscience.iop.org/article/10.1143/JJAP.44.8269/meta; jsessionid= C543F8FDFDCEAB28B0E6AB280CCF78FB.c4. iopscience.cld.iop. org.

[6] H. Kamisaka and K. Yamashita. Theoretical Study of the Interstitial Oxygen Atom in Anatase and Rutile $\mathrm{TiO}_{2}$ : Electron Trapping and Elongation of the r(O-O) Bond. The Journal of Physical Chemistry C (2011) 115(16) 82658273. doi:10.1021/jp110648q. URL http://pubs.acs.org/doi/abs/10.1021/ jp110648q.

[7] A. Fujishima and K. Honda. Electrochemical photolysis of water at a semiconductor electrode. nature (1972) 238(5358) 37-38. doi:10.1038/ 238037a0. URL https://www.nature.com/nature/journal/v238/n5358/ abs/238037a0.html. 
[8] Wikipedia, the free encyclopedia. Hydrogen (October 2017). URL https://en . wikipedia.org/wiki/Hydrogen.

[9] P. Salvador, M. L. Garcia Gonzalez and F. Munoz. Catalytic role of lattice defects in the photoassisted oxidation of water at (001) n-titanium(IV) oxide rutile. The Journal of Physical Chemistry (1992) 96(25) 10349-10353. doi:10. 1021/j100204a046. URL http://pubs.acs.org/doi/10.1021/j100204a046.

[10] N. Yamamoto, S. Tonomura, T. Matsuoka and H. Tsubomura. A study on a palladium-titanium oxide Schottky diode as a detector for gaseous components. Surface Science (1980) 92(2) 400-406. doi:10.1016/0039-6028(80)90212-5. URL http://www.sciencedirect.com/science/article/pii/0039602880902125.

[11] H. Kobayashi, K. Kishimoto and Y. Nakato. Reactions of hydrogen at the interface of palladium-titanium dioxide Schottky diodes as hydrogen sensors, studied by workfunction and electrical characteristic measurements. Surface Science (1994) 306(3) 393-405. doi:10.1016/0039-6028(94)90080-9. URL http: //www.sciencedirect.com/science/article/pii/0039602894900809.

[12] M. Cerchez, H. Langer, M. E. Achhab, T. Heinzel, D. Ostermann, H. Lüder and J. Degenhardt. Dynamics of hydrogen sensing with Pt/TiO $\mathrm{O}_{2}$ Schottky diodes. Applied Physics Letters (jul 2013) 103(3) 033522. doi:10.1063/1.4816265. URL http://aip.scitation.org/doi/10.1063/1.4816265.

[13] M. Strungaru, M. Cerchez, S. Herbertz, T. Heinzel, M. E. Achhab and K. Schierbaum. Interdependence of electroforming and hydrogen incorporation in nanoporous titanium dioxide. Applied Physics Letters (2015) 106(14) 143109. doi:10.1063/1.4917034. URL http://aip.scitation.org/doi/10. $1063 / 1.4917034$.

[14] Wikipedia, the free encyclopedia. CPK coloring (November 2017). URL https: //en.wikipedia.org/wiki/CPK_coloring.

[15] W. Borchardt-Ott and H. Sowa. Kristallographie. Springer Berlin Heidelberg, 8 edition (2013). ISBN 978-3-642-34811-2. doi:10.1007/978-3-642-34811-2. URL https://link. springer.com/book/10.1007/978-3-642-34811-2.

[16] R. Alsfasser, C. Janiak, T. M. Klapötke and H.-J. Meyer. Riedel Moderne Anorganische Chemie. Walter de Gruyter, Berlin, third edition (2007). ISBN 978-311-019060-1. URL https://www. degruyter.com/view/product/128059.

[17] J. L. Murray and H. A. Wriedt. The O-Ti (Oxygen-Titanium) system. Journal of Phase Equilibria (Apr 1987) 8(2) 148-165. doi:10.1007/BF02873201. URL https://link.springer. com/article/10.1007\%2FBF02873201?LI=true. 
[18] H. Okamoto. O-Ti (Oxygen-Titanium). Journal of Phase Equilibria and Diffusion (Jul 2011) 32(5) 473. doi:10.1007/s11669-011-9935-5. URL https: //link. springer. com/article/10.1007\%2Fs11669-011-9935-5.

[19] P. G. Wahlbeck and P. W. Gilles. Reinvestigation of the Phase Diagram for the System Titanium-Oxygen. Journal of the American Ceramic Society (1966) 49(4) 180-183. doi:10.1111/j.1151-2916.1966.tb13229. x. URL http://onlinelibrary.wiley.com/doi/10.1111/j.1151-2916. 1966.tb13229.x/abstract.

[20] S. Andersson, B. Collén, U. Kuylenstierna and A. Magnéli. Phase analysis studies on the titanium-oxygen system. Acta Chemica Scandinavica (1957) 11(10) 1641-1652. URL http://actachemscand. dk/volume.php?select1=2\&vol=11.

[21] O. T. Sorensen. Nonstoichiometric Oxides (Materials science and technology). Academic Press (1981). ISBN 978-0-12-655280-5. URL http://www . sciencedirect.com/science/book/9780126552805.

[22] E. Stoyanov, F. Langenhorst and G. Steinle-Neumann. The effect of valence state and site geometry on $\mathrm{Ti} \mathrm{L}_{3,2}$ and $\mathrm{O} \mathrm{K}$ electron energy-loss spectra of $\mathrm{Ti}_{x} \mathrm{O}_{y}$ phases. American Mineralogist (apr 2007) 92(4) 577-586. doi:10.2138/am.2007. 2344. URL http://ammin.geoscienceworld.org/content/92/4/577.

[23] F. A. Grant. Properties of Rutile (Titanium Dioxide). Rev. Mod. Phys. (Jul 1959) 31 646-674. doi:10.1103/RevModPhys.31.646. URL https://link.aps. org/doi/10.1103/RevModPhys.31.646.

[24] M. K. Nowotny, T. Bak and J. Nowotny. Electrical Properties and Defect Chemistry of $\mathrm{TiO}_{2}$ Single Crystal. III. Equilibration Kinetics and Chemical Diffusion. The Journal of Physical Chemistry B (2006) 110(33) 16292-16301. doi:10.1021/ jp060623k. URL http://pubs.acs.org/doi/abs/10.1021/jp060623k.

[25] M. K. Nowotny, T. Bak, J. Nowotny and C. C. Sorrell. Titanium vacancies in nonstoichiometric $\mathrm{TiO}_{2}$ single crystal. physica status solidi (b) (2005) 242(11) R88-R90. doi:10.1002/pssb.200541186. URL http://onlinelibrary.wiley. com/doi/10.1002/pssb.200541186/abstract.

[26] M. K. Nowotny, T. Bak and J. Nowotny. Electrical Properties and Defect Chemistry of $\mathrm{TiO}_{2}$ Single Crystal. IV. Prolonged Oxidation Kinetics and Chemical Diffusion. The Journal of Physical Chemistry B (2006) 110(33) 16302-16308. doi:10. 1021/jp060624c. URL http://pubs.acs.org/doi/abs/10.1021/jp060624c.

[27] U. Diebold. The surface science of titanium dioxide. Surface Science Reports (jan 2003) 48(5-8) 53-229. doi:10.1016/s0167-5729(02)00100-0. URL http: //www.sciencedirect.com/science/article/pii/S0167572902001000. 
[28] D. T. Cromer and K. Herrington. The Structures of Anatase and Rutile. Journal of the American Chemical Society (1955) 77(18) 4708-4709. doi:10.1021/ ja01623a004. URL http://pubs.acs.org/doi/abs/10.1021/ja01623a004.

[29] A. Imanishi, E. Tsuji and Y. Nakato. Dependence of the Work Function of TiO (Rutile) on Crystal Faces, Studied by a Scanning Auger Microprobe. The Journal of Physical Chemistry C (2007) 111(5) 2128-2132. doi:10.1021/jp0668403. URL http://pubs.acs.org/doi/abs/10.1021/jp0668403.

[30] M. K. Nowotny, T. Bak and J. Nowotny. Electrical Properties and Defect Chemistry of $\mathrm{TiO}_{2}$ Single Crystal. I. Electrical Conductivity. The Journal of Physical Chemistry B (2006) 110(33) 16270-16282. doi:10.1021/jp0606210. URL http://pubs.acs.org/doi/abs/10.1021/jp0606210.

[31] L. Kavan, M. GrÃ atzel, S. E. Gilbert, C. Klemenz and H. J. Scheel. Electrochemical and Photoelectrochemical Investigation of Single-Crystal Anatase. Journal of the American Chemical Society (1996) 118(28) 6716-6723. doi: 10.1021/ja954172l. URL http://pubs .acs.org/doi/abs/10.1021/ja9541721.

[32] T. Bak, J. Nowotny, M. Rekas and C. Sorrell. Defect chemistry and semiconducting properties of titanium dioxide: III. Mobility of electronic charge carriers. Journal of Physics and Chemistry of Solids (2003) 64(7) 1069-1087. doi:10.1016/S0022-3697(02)00481-X. URL http://www.sciencedirect.com/ science/article/pii/S002236970200481X.

[33] T. Bak, J. Nowotny, M. Rekas and C. Sorrell. Defect chemistry and semiconducting properties of titanium dioxide: I. Intrinsic electronic equilibrium. Journal of Physics and Chemistry of Solids (2003) 64(7) 1043-1056. doi:10.1016/S0022-3697(02)00479-1. URL http://www.sciencedirect.com/ science/article/pii/S0022369702004791.

[34] K. Momma and F. Izumi. VESTA 3 for three-dimensional visualization of crystal, volumetric and morphology data. Journal of Applied Crystallography (Dec 2011) 44(6) 1272-1276. doi:10.1107/S0021889811038970. URL http: //scripts.iucr.org/cgi-bin/paper?S0021889811038970.

[35] R. Brydson, H. Sauer, W. Engel, J. M. Thomass, E. Zeitler, N. Kosugi and H. Kuroda. Electron energy loss and X-ray absorption spectroscopy of rutile and anatase: a test of structural sensitivity. Journal of Physics: Condensed Matter (1989) 1(4) 797. URL http://stacks . iop.org/0953-8984/1/i=4/a=012.

[36] D. W. Fischer. Molecular-Orbital Interpretation of the Soft X-Ray LII,III Emission and Absorption Spectra from Some Titanium and Vanadium Compounds. Journal of Applied Physics (1970) 41(9) 3561-3569. doi:10.1063/1.1659472. URL http://aip.scitation.org/doi/abs/10.1063/1.1659472. 
[37] S. O. Kucheyev, T. van Buuren, T. F. Baumann, J. H. Satcher, T. M. Willey, R. W. Meulenberg, T. E. Felter, J. F. Poco, S. A. Gammon and L. J. Terminello. Electronic structure of titania aerogels from soft x-ray absorption spectroscopy. Phys. Rev. B (Jun 2004) 69 245102. doi:10.1103/PhysRevB.69.245102. URL https://journals.aps.org/prb/abstract/10.1103/PhysRevB.69.245102.

[38] D. B. Williams and C. B. Carter. Transmission Electron Microscopy. Springer US, 2 edition (2009). ISBN 978-0-387-76500-6. doi:10.1007/978-0-387-76501-3. URL https://link. springer.com/book/10.1007\%2F978-0-387-76501-3.

[39] R. F. Egerton. Electron Energy-Loss Spectroscopy in the Electron Microscope. Springer-Verlag GmbH (2011). ISBN 144199582X. doi: 10.1007/978-1-4419-9583-4. URL https://www.springer.com/de/book/ 9781441995827.

[40] R. F. Egerton. Electron energy-loss spectroscopy in the TEM. Reports on Progress in Physics (2009) 72 016502. doi:10.1088/0034-4885/72/1/016502. URL http://iopscience.iop.org/article/10.1088/0034-4885/72/1/016502/ meta; jsessionid=F9A5A98ABC84EF6E9B35426575C728A3.c3. iopscience. cld.iop.org.

[41] EELS Atlas (2017.06.27). URL http://www.eels.info/atlas/titanium.

[42] R. Brydson, B. Williams, W. Engel, H. Sauer, E. Zeitler and J. Thomas. Electron energy-loss spectroscopy (EELS) and the electronic structure of titanium dioxide. Solid State Communications (1987) 64(4) 609-612. doi:10. 1016/0038-1098(87)90792-7. URL http://www.sciencedirect.com/science/ article/pii/0038109887907927.

[43] M. Cheynet, S. Pokrant, S. Irsen and P. Krüger. New fine structures resolved at the ELNES Ti-L $L_{2,3}$ edge spectra of anatase and rutile: Comparison between experiment and calculation. Ultramicroscopy (2010) 110(8) 1046-1053. doi:10. 1016/j.ultramic.2010.03.001. URL http://www.sciencedirect.com/science/ article/pii/S0304399110000793.

[44] P. Krüger. Multichannel multiple scattering calculation of $L_{2,3}$-edge spectra of $\mathrm{TiO}_{2}$ and $\mathrm{SrTiO}_{3}$ : Importance of multiplet coupling and band structure. Phys. Rev. B (Mar 2010) 81 125121. doi:10.1103/PhysRevB.81.125121. URL https: //journals.aps .org/prb/abstract/10.1103/PhysRevB.81.125121.

[45] M. Robinson, N. Marks and G. Lumpkin. Structural dependence of threshold displacement energies in rutile, anatase and brookite $\mathrm{TiO}_{2}$. Materials Chemistry and Physics (sep 2014) 147(1-2) 311-318. doi:10.1016/j.matchemphys. 2014.05.006. URL http://www.sciencedirect.com/science/article/pii/ S0254058414003058. 
[46] R. Egerton, P. Li and M. Malac. Radiation damage in the TEM and SEM. Micron (aug 2004) 35(6) 399-409. doi:10.1016/j.micron.2004.02.003. URL http: //www.sciencedirect.com/science/article/pii/S0968432804000381. International Wuhan Symposium on Advanced Electron Microscopy.

[47] W. Wallace, Q. Zhong, J. Genzer, R. Composto and D. Bonnell. On the use of ion scattering to examine the role of hydrogen in the reduction of $\mathrm{TiO}_{2}$. Journal of Materials Research (1993) 8(7) 1629-1634. URL https://doi.org/10. 1557/JMR . 1993. 1629.

[48] J. He, R. Behera, M. Finnis, X. Li, E. Dickey, S. Phillpot and S. Sinnott. Prediction of high-temperature point defect formation in $\mathrm{TiO}_{2}$ from combined ab initio and thermodynamic calculations. Acta Materialia (2007) 55(13) 43254337. doi:10.1016/j.actamat.2007.04.005. URL http://www.sciencedirect. com/science/article/pii/S1359645407002406.

[49] H. Peng. First-principles study of native defects in rutile $\mathrm{TiO}_{2}$. Physics Letters A (2008) 372(9) 1527-1530. doi:10.1016/j.physleta.2007.10.011. URL http: //www.sciencedirect.com/science/article/pii/S0375960107014387.

[50] T. S. Bjørheim, A. Kuwabara and T. Norby. Defect Chemistry of Rutile $\mathrm{TiO}_{2}$ from First Principles Calculations. The Journal of Physical Chemistry C (2013) 117(11) 5919-5930. doi:10.1021/jp304146e. URL http://pubs.acs.org/doi/ abs/10.1021/jp304146e.

[51] M. K. Nowotny, T. Bak and J. Nowotny. Electrical Properties and Defect Chemistry of $\mathrm{TiO}_{2}$ Single Crystal. II. Thermoelectric Power. The Journal of Physical Chemistry B (2006) 110(33) 16283-16291. doi:10.1021/jp060622s. URL http://pubs.acs.org/doi/abs/10.1021/jp060622s.

[52] J. Nowotny. Effect of Hydrogen on Semiconducting Properties of $\mathrm{TiO}_{2}$ Single Crystal. Jonker Analysis. The Journal of Physical Chemistry C (2011) 115(37) 18316-18326. doi:10.1021/jp204072z. URL http://pubs.acs.org/doi/abs/ 10.1021/jp204072z.

[53] T. Bak, J. Nowotny, M. Rekas and C. Sorrell. Defect chemistry and semiconducting properties of titanium dioxide: II. Defect diagrams. Journal of Physics and Chemistry of Solids (2003) 64(7) 1057-1067. doi:10.1016/ S0022-3697(02)00480-8. URL http://www.sciencedirect.com/science/ article/pii/S0022369702004808.

[54] J.-L. Carpentier, A. Lebrun and F. Perdu. Electronic conduction in pure and chromium-doped rutile at $1273 \mathrm{~K} . \quad$ Le Journal de Physique Colloques (1986) 47(C1) C1-819. doi:10.1051/jphyscol:19861125. 
URL https://jphyscol.journaldephysique.org/articles/jphyscol/abs/ 1986/01/jphyscol198647C1125/jphyscol198647C1125.html.

[55] J. Nowotny, T. Bak, M. K. Nowotny and L. R. Sheppard. Chemical diffusion in metal oxides. Example of $\mathrm{TiO}_{2}$. Ionics (Sep 2006) 12(3) 227-243. doi: 10.1007/s11581-006-0036-0. URL https://link. springer.com/article/10. $1007 / \mathrm{s} 11581-006-0036-0$.

[56] P. F. Chester and D. H. Bradhurst. Electrolytically Induced Conductivity in Rutile. Nature (1963) 199 1056-1057. doi:10.1038/1991056a0. URL https: //www . nature.com/nature/journal/v199/n4898/abs/1991056a0.html.

[57] F. Filippone, G. Mattioli, P. Alippi and A. Amore Bonapasta. Properties of hydrogen and hydrogen-vacancy complexes in the rutile phase of titanium dioxide. Phys. Rev. B (Dec 2009) 80 245203. doi:10.1103/PhysRevB.80.245203. URL https://link.aps.org/doi/10.1103/PhysRevB.80.245203.

[58] O. W. Johnson, J. DeFord and J. W. Shaner. Experimental technique for the precise determination of $\mathrm{H}$ and $\mathrm{D}$ concentration in rutile $\left(\mathrm{TiO}_{2}\right)$. Journal of Applied Physics (1973) 44(7) 3008-3012. doi:10.1063/1.1662697. URL http: //aip.scitation.org/doi/abs/10.1063/1.1662697.

[59] F. Herklotz, E. V. Lavrov and J. Weber. Infrared absorption of the hydrogen donor in rutile $\mathrm{TiO}_{2}$. Phys. Rev. B (Jun 2011) 83 235202. doi:10.1103/ PhysRevB.83.235202. URL https://link.aps.org/doi/10.1103/PhysRevB. 83. 235202.

[60] H. Nakatsuji and M. Hada. Interaction of a hydrogen molecule with palladium. Journal of the American Chemical Society (1985) 107(26) 82648266. doi:10.1021/ja00312a078. URL http://pubs.acs.org/doi/abs/10. $1021 / \mathrm{ja} 00312 \mathrm{a} 078$.

[61] W. M. Bartczak and J. Stawowska. Interaction of Dihydrogen with Transition Metal (Pd, Ni, Ag, Cu) Clusters. Structural Chemistry (2004) 15(5) 447-459. doi:10.1023/B:STUC.0000037902.93420.28. URL https://link. springer.com/article/10.1023/B:STUC.0000037902.93420.28.

[62] O. W. Johnson, S.-H. Paek and J. W. DeFord. Diffusion of $H$ and $D$ in $\mathrm{TiO}_{2}$ : Suppression of internal fields by isotope exchange. Journal of Applied Physics (mar 1975) 46(3) 1026-1033. doi:10.1063/1.322206. URL http: //aip.scitation.org/doi/10.1063/1.322206.

[63] T. Norby. Proton Conduction in Solids: Bulk and Interfaces. MRS Bulletin (2009) 34(12) 923-928. doi:10.1557/mrs2009.214. URL https://www.cambridge.org/core/journals/mrs-bulletin/ 
article/proton-conduction-in-solids-bulk-and-interfaces/ 29F8D17BB4F56C723F141B4392D4E706.

[64] S. Erdal, C. Kongshaug, T. S. BjÃ sheim, N. Jalarvo, R. Haugsrud and T. Norby. Hydration of Rutile $\mathrm{TiO}_{2}$ : Thermodynamics and Effects on $n$ - and p-Type Electronic Conduction. The Journal of Physical Chemistry C (2010) 114(19) 91399145. doi:10.1021/jp101886a. URL http://pubs.acs.org/doi/abs/10.1021/ jp101886a.

[65] J. Nowotny, T. Bak, M. Ionescu and M. A. Alim. Electrical Properties and Defect Chemistry of In-Doped $\mathrm{TiO}_{2}$ in Terms of the Jonker Formalism. The Journal of Physical Chemistry A (2015) 119(17) 4032-4040. doi:10.1021/acs.jpca.5b01368. URL http://pubs.acs.org/doi/abs/10.1021/acs.jpca.5b01368.

[66] Wikipedia, the free encyclopedia. Luft (October 2017). URL https://de. wikipedia.org/wiki/Luft.

[67] Y. Chen, R. Gonzalez and K. L. Tsang. Diffusion of Deuterium and Hydrogen in Rutile $\mathrm{TiO}_{2}$ Crystals at Low Temperatures. Phys. Rev. Lett. (Sep 1984) 53 1077-1079. doi:10.1103/PhysRevLett.53.1077. URL https://link.aps.org/ doi/10.1103/PhysRevLett.53.1077.

[68] E. J. Spahr, L. Wen, M. Stavola, L. A. Boatner, L. C. Feldman, N. H. Tolk and G. Lüpke. Giant Enhancement of Hydrogen Transport in Rutile $\mathrm{TiO}_{2}$ at Low Temperatures. Phys. Rev. Lett. (May 2010) 104205901. doi:10.1103/PhysRevLett.104.205901. URL https://link.aps.org/doi/10 . 1103/PhysRevLett. 104.205901.

[69] A. L. Samgin and A. N. Ezin. Room-temperature proton-hopping transport in rutile-type oxides in the field of resonant laser radiation. Technical Physics Letters (Mar 2014) 40(3) 252-255. doi:10.1134/S1063785014030262. URL https://link.springer.com/article/10.1134\%2FS1063785014030262.

[70] J. B. Bates, J. C. Wang and R. A. Perkins. Mechanisms for hydrogen diffusion in $\mathrm{TiO}_{2}$. Phys. Rev. B (Apr 1979) 19 4130-4139. doi:10.1103/PhysRevB.19.4130. URL https://link.aps.org/doi/10.1103/PhysRevB.19.4130.

[71] M. I. Nandasiri, V. Shutthanandan, S. Manandhar, A. M. Schwarz, L. Oxenford, J. V. Kennedy, S. Thevuthasan and M. A. Henderson. Instability of Hydrogenated $\mathrm{TiO}_{2}$. The Journal of Physical Chemistry Letters (2015) 6(22) 4627-4632. doi: 10.1021/acs.jpclett.5b02219. URL http://pubs.acs.org/doi/10.1021/acs . jpclett.5b02219.

[72] J. W. Arblaster. Crystallographic properties of palladium. Platinum Metals Review (2012) 56(3) 181-189. doi:10.1595/147106712X646113. URL 
http://www . ingentaconnect. com/content/matthey/pmr/2012/00000056/ $00000003 /$ art00006.

[73] T. Hahn, editor. International Tables for Crystallography, volume A. JOHN WILEY \& SONS INC, fifth edition (2005). ISBN 978-0-470-68911-0. URL http://eu.wiley.com/WileyCDA/WileyTitle/productCd-0470689110.html.

[74] L. A. DuBridge and W. W. Roehr. Photoelectric and Thermionic Properties of Palladium. Phys. Rev. (Jan 1932) 39 99-107. doi:10.1103/PhysRev.39.99. URL https://journals.aps.org/pr/abstract/10.1103/PhysRev.39.99.

[75] G. L. Holleck. Diffusion and solubility of hydrogen in palladium and palladium-silver alloys. The Journal of Physical Chemistry (1970) 74(3) 503511. doi:10.1021/j100698a005. URL http://pubs .acs .org/doi/abs/10.1021/ j100698a005? journalCode=jpchax.

[76] R. D. Deslattes, E. G. Kessler, P. Indelicato, L. de Billy, E. Lindroth and J. Anton. X-ray transition energies: new approach to a comprehensive evaluation. Rev. Mod. Phys. (Jan 2003) 75 35-99. doi:10.1103/RevModPhys.75.35. URL https://link.aps.org/doi/10.1103/RevModPhys.75.35.

[77] J. H. Hubbell and S. M. Seltzer. Tables of X-Ray Mass Attenuation Coefficients and Mass Energy-Absorption Coefficients (version 1.4) (2004). URL http:// physics.nist.gov/xaamdi.

[78] Bruker AXS GmbH. Ecole de Réflectivité given by Dr. Martin Zimmermann (2014).

[79] F. D. Manchester, A. San-Martin and J. M. Pitre. The H-Pd (hydrogenpalladium) System. Journal of Phase Equilibria (1994) 15(1) 62-83. doi: 10.1007/BF02667685. URL https://link.springer.com/article/10.1007\% 2FBF02667685?LI=true.

[80] G. Alefeld and J. Völkl. Hydrogen in Metals II. Springer-Verlag Berlin Heidelberg (1978).

[81] S. Wagner. Dünne Palladium-Wasserstoff-Schichten als Modellsystem: Thermodynamik struktureller Phasenübergänge unter elastischen und mikrostrukturellen Zwangsbedingungen. Ph.D. thesis, Institut für Materialphysik, GeorgAugust-Universität Göttingen (2014). URL http://hdl .handle.net/11858/ 00-1735-0000-0022-6048-1.

[82] J. G. Early. Hydrogen diffusion in palladium by galvanostatic charging. Acta Metallurgica (1978) 26(8) 1215-1223. doi:10.1016/0001-6160(78)90005-6. URL http://www.sciencedirect.com/science/article/pii/0001616078900056. 
[83] D. R. Hummer, P. J. Heaney and J. E. Post. Thermal expansion of anatase and rutile between 300 and $575 \mathrm{~K}$ using synchrotron powder X-ray diffraction. Powder Diffraction (2007) 22(4) 352-357. URL https://doi.org/10.1154/ 1.2790965 .

[84] M. Ohring. Materials Science of Thin Films. Academic Press, second edition edition (2002). ISBN 9780080491783. URL https://www.elsevier.com/books/ materials-science-of-thin-films/ohring/978-0-12-524975-1.

[85] D. Sander. The correlation between mechanical stress and magnetic anisotropy in ultrathin films. Reports on Progress in Physics (1999) 62(5) 809-858. doi: 10.1088/0034-4885/62/5/204. URL http://iopscience.iop.org/article/ $10.1088 / 0034-4885 / 62 / 5 / 204$.

[86] D. K. Hsu and R. G. Leisure. Elastic constants of palladium and $\beta$-phase palladium hydride between 4 and 300 K. Physical Review B (1979) 201339 1344. doi:10.1103/PhysRevB.20.1339. URL https://journals.aps.org/prb/ abstract/10.1103/PhysRevB.20.1339.

[87] J. A. Rayne. Elastic Constants of Palladium from 4.2-300 K. Physical Review (1960) 118 1545-1549. doi:10.1103/PhysRev.118.1545. URL https: //journals.aps.org/pr/abstract/10.1103/PhysRev.118.1545.

[88] W. Mönch. Electronic Properties of Semiconductor Interfaces. SpringerVerlag Berlin Heidelberg (2004). ISBN 978-3-540-20215-8. doi: 10.1007/978-3-662-06945-5. URL https://www.springer.com/la/book/ 9783540202158.

[89] R. T. Tung. The physics and chemistry of the Schottky barrier height. Applied Physics Reviews (2014) 1 011304. doi:10.1063/1.4858400. URL http://aip. scitation.org/doi/10.1063/1.4858400.

[90] G. A. Hope and A. J. Bard. Platinum/titanium dioxide (rutile) interface. Formation of ohmic and rectifying junctions. The Journal of Physical Chemistry (1983) 87(11) 1979-1984. doi:10.1021/j100234a029. URL http://pubs.acs. org/doi/10.1021/j100234a029.

[91] V. Burlaka, S. Wagner, M. Hamm and A. Pundt. Suppression of Phase Transformation in Nb-H Thin Films below Switchover Thickness. Nano Letters (2016) 16(10) 6207-6212. doi:10.1021/acs.nanolett.6b02467. URL http: //pubs.acs.org/doi/abs/10.1021/acs. nanolett.6b02467.

[92] P. Kelly and R. Arnell. Magnetron sputtering: a review of recent developments and applications. Vacuum (mar 2000) 56(3) 159-172. doi:10.1016/ 
Bibliography

s0042-207x(99)00189-x. URL http://www.sciencedirect.com/science/ article/pii/S0042207X9900189X.

[93] N. Herwig. Optimierung von Titandioxid/Palladium Grenzflächen für Elektronenenergieverlustspektroskopie Studien. bachelor thesis, Institut für Materialphysik, Georg-August-Universität Göttingen (2015).

[94] R. Shimizu, T. Hitosugi, K. S. Nakayama, T. Sakurai, M. Shiraiwa, T. Hasegawa and T. Hashizume. Preparation of atomically flat $\mathrm{TiO}_{2}(110)$ substrate. Japanese Journal of Applied Physics (2009) 48(12R) 125506. URL http://stacks.iop. org $/ 1347-4065 / 48 / i=12 R / a=125506$.

[95] R. Nakamura, N. Ohashi, A. Imanishi, T. Osawa, Y. Matsumoto, H. Koinuma and Y. Nakato. Crystal-Face Dependences of Surface Band Edges and Hole Reactivity, Revealed by Preparation of Essentially Atomically Smooth and Stable (110) and (100) n- $\mathrm{TiO}_{2}$ (Rutile) Surfaces. The Journal of Physical Chemistry B (feb 2005) 109(5) 1648-1651. doi:10.1021/jp044710t. URL http://pubs.acs . org/doi/abs/10.1021/jp044710t.

[96] J. B. Wagner, F. Cavalca, C. D. Damsgaard, L. D. L. Duchstein and T. W. Hansen. Exploring the environmental transmission electron microscope. Micron (2012) 43(11) 1169-1175. doi:10.1016/j.micron.2012.02.008. URL https : //www . sciencedirect.com/science/article/pii/S0968432812000510.

[97] M. Süess, E. Mueller and R. Wepf. Minimization of amorphous layer in Ar ${ }^{+}$ ion milling for UHR-EM. Ultramicroscopy (jul 2011) 111(8) 1224-1232. doi:10. 1016/j.ultramic.2011.03.004. URL http://www.sciencedirect.com/science/ article/pii/S0304399111000908.

[98] T. W. Hansen, J. B. Wagner and R. E. Dunin-Borkowski. Aberration corrected and monochromated environmental transmission electron microscopy: challenges and prospects for materials science. Materials Science and Technology (2010) 26(11) 1338-1344. doi:10.1179/026708310X12756557336355. URL http://www . tandf online.com/doi/abs/10.1179/026708310X12756557336355.

[99] Gatan, Inc. DigitalMicrograph Manual (2015).

[100] P. D. G. Kothleitner. Workshop: EELS advanced course (December 2015). Göttingen.

[101] P. C. Tiemeijer, J. H. A. van Lin, B. H. Freitag and A. F. de Jong. Monochromized 200kV (S)TEM. Microscopy and Microanalysis (2002) 8(S02) 70-71. doi:10.1017/S143192760210184X. URL https: //www . cambridge.org/core/journals/microscopy-and-microanalysis/ article/monochromized-200kv-stem/098D794A31AD1E502742D5578EEFCD4C. 
[102] The MathWorks, Inc. MATLAB Documentation (2017.12.06). URL https://www.mathworks.com/help/optim/ug/lsqcurvefit.html?s_tid= gn_loc_drop.

[103] R. W. Siegel. Positron Annihilation Spectroscopy. Annual Review of Materials Science (1980) 10(1) 393-425. doi:10.1146/annurev.ms.10.080180. 002141. URL http://www . annualreviews.org/doi/abs/10.1146/annurev . ms. 10.080180 .002141$.

[104] J. Č́žek. Characterization of lattice defects in metallic materials by positron annihilation spectroscopy: A review. Journal of Materials Science \& Technology (2017) doi:10.1016/j.jmst.2017.11.050. URL https://www . sciencedirect. com/science/article/pii/S1005030217303225.

[105] P. J. Schultz and K. G. Lynn. Interaction of positron beams with surfaces, thin films, and interfaces. Rev. Mod. Phys. (Jul 1988) 60 701-779. doi:10.1103/RevModPhys.60.701. URL https://link.aps.org/doi/10.1103/ RevModPhys.60.701.

[106] W. Anwand, G. Brauer, M. Butterling, H. R. Kissener and A. Wagner. Design and Construction of a Slow Positron Beam for Solid and Surface Investigations. Defect and Diffusion Forum (2012) 331 25-40. doi:10.4028/www.scientific.net/ DDF.331.25. URL https://www.scientific.net/DDF.331.25.

[107] R. Krause-Rehberg and H. S. Leipner. Positron Annihilation in Semiconductors: Defect Studies. Springer-Verlag Berlin Heidelberg (1999). ISBN 978-3-540-643715 .

[108] L. Spieß, G. Teichert, R. Schwarzer, H. Behnken and C. Genzel. Moderne Röntgenbeugung: Röntgendiffraktometrie für Materialwissenschaftler, Physiker und Chemiker (German Edition). Vieweg+Teubner Verlag (2009). ISBN 9783835101661.

[109] W. L. Bragg. The structure of some crystals as indicated by their diffraction of X-rays. In Proceedings of the Royal Society of London A: Mathematical, Physical and Engineering Sciences, volume 89. The Royal Society (1913) 248-277. doi: 10.1098/rspa.1913.0083. URL http://rspa.royalsocietypublishing.org/ content/89/610/248.

[110] M. Dornheim. Spannungen, Dehnungen und Lage der Phasengrenzen in dünnen $\mathrm{Nb}$ - und Y-Schichten bei Wasserstoffbe- und -entladung. Ph.D. thesis, GeorgAugust-Universität zu Göttingen (2002).

[111] T. Harumoto, Y. Ohnishi, K. Nishio, T. Ishiguro, J. Shi and Y. Nakamura. In-situ X-ray diffraction study of hydrogen absorption and desorption processes 
in Pd thin films: Hydrogen composition dependent anisotropic expansion and its quantitative description. AIP Advances (2017) 7(6) 065108. doi:10.1063/1. 4986214. URL http://aip.scitation.org/doi/10.1063/1.4986214.

[112] J. F. Watts and J. Wolstenholme. An Introduction to Surface Analysis by $X P S$ and AES. John Wiley \& Sons, Ltd (2005). ISBN 9780470867938. doi:10.1002/0470867930. URL http://onlinelibrary.wiley.com/book/10. 1002/0470867930.

[113] G. Binnig, C. F. Quate and C. Gerber. Atomic Force Microscope. Phys. Rev. Lett. (Mar 1986) 56 930-933. doi:10.1103/PhysRevLett.56.930. URL https: //journals.aps.org/prl/abstract/10.1103/PhysRevLett.56.930.

[114] G. Haugstad. Atomic Force Microscopy: Understanding Basic Modes and Advanced Applications. John Wiley \& Sons (2012). ISBN 9781118360668. doi: 10.1002/9781118360668. URL http://onlinelibrary.wiley.com/book/10 . 1002/9781118360668.

[115] D. Nečas and P. Klapetek. Gwyddion: an open-source software for SPM data analysis. Central European Journal of Physics (2012) 10 181-188. doi: 10.2478/s11534-011-0096-2. URL https: //www. degruyter.com/view/j/phys . 2012.10. issue-1/s11534-011-0096-2/s11534-011-0096-2.xml.

[116] P. Klapetek, D. Nečas and C. Anderson. Gwyddion user guide (2017). URL http://gwyddion. net/documentation/user-guide-en/.

[117] I. Oxford Instruments Asylum Research. MFP-3D specifications (201708-19). URL https://www.asylumresearch.com/Products/Mfp3DSA/ Mfp3DSAProduct.shtml\#Specifications.

[118] I. Oxford Instruments Asylum Research. AC160TS probe specifications (2017.08.23). URL https://afmprobes.asylumresearch.com/ac160ts-r3. html.

[119] P. Hohenberg and W. Kohn. Inhomogeneous Electron Gas. Physical Review (1964) 136 B864-B871. doi:10.1103/PhysRev.136.B864. URL https: //journals.aps.org/pr/abstract/10.1103/PhysRev.136.B864.

[120] E. Schrödinger. Quantisierung als Eigenwertproblem. Annalen der Physik (1926) 385(13) 437-490. doi:10.1002/andp.19263851302. URL http:// onlinelibrary.wiley.com/doi/10.1002/andp.19263851302/full.

[121] P. E. Blöchl. Projector augmented-wave method. Phys. Rev. B (Dec 1994) 50 17953-17979. doi:10.1103/PhysRevB.50.17953. URL https://journals.aps . org/prb/abstract/10.1103/PhysRevB.50.17953. 
[122] M. Sotoudeh, S. Rajpurohit, P. Blöchl, D. Mierwaldt, J. Norpoth, V. Roddatis, S. Mildner, B. Kressdorf, B. Ifland and C. Jooss. Electronic structure of $\mathrm{Pr}_{1-x} \mathrm{Ca}_{x} \mathrm{MnO}_{3}$. Phys. Rev. B (Jun 2017) 95 235150. doi:10.1103/ PhysRevB.95.235150. URL https://journals.aps.org/prb/abstract/10. 1103/PhysRevB.95.235150.

[123] M. Sotoudeh. Ph.D. thesis. Georg-August-Universität Göttingen, To be published.

[124] W. Göpel, J. Anderson, D. Frankel, M. Jaehnig, K. Phillips, J. Schäfer and G. Rocker. Surface defects of TiO $2(110):$ A combined XPS, $X A E S$ and ELS study. Surface Science (1984) 139(2) 333-346. doi:10. 1016/0039-6028(84)90054-2. URL http://www. sciencedirect.com/science/ article/pii/0039602884900542.

[125] N. C. Saha and H. G. Tompkins. Titanium nitride oxidation chemistry: An $x$-ray photoelectron spectroscopy study. Journal of Applied Physics (1992) 72(7) 3072-3079. doi:10.1063/1.351465. URL http://aip.scitation.org/doi/10. 1063/1.351465.

[126] K. A. Stoerzinger, W. T. Hong, E. J. Crumlin, H. Bluhm, M. D. Biegalski and Y. Shao-Horn. Water Reactivity on the $\mathrm{LaCoO}_{3}$ (001) Surface: An Ambient Pressure X-ray Photoelectron Spectroscopy Study. The Journal of Physical Chemistry C (2014) 118(34) 19733-19741. doi:10.1021/jp502970r. URL http://pubs.acs.org/doi/abs/10.1021/jp502970r.

[127] M. Litschauer. Optimierung des Wachstums von Palladiumschichten auf RutilTitandioxideinkristallen. bachelor thesis, Institut für Materialphysik, GeorgAugust-Universität Göttingen (2017).

[128] J. de Heuvel. Elektrische Charakterisierung der Grenzfläche zwischen Palladium und Titandioxid als Metall-Halbleiter-Kontakt. bachelor thesis, Institut für Materialphysik, Georg-August-Universität Göttingen (2016).

[129] M. Li, W. Hebenstreit, U. Diebold, A. M. Tyryshkin, M. K. Bowman, G. G. Dunham and M. A. Henderson. The Influence of the Bulk Reduction State on the Surface Structure and Morphology of Rutile TiO2(110) Single Crystals. The Journal of Physical Chemistry B (2000) 104(20) 4944-4950. doi:10.1021/ jp9943272. URL http://pubs.acs.org/doi/abs/10.1021/jp9943272.

[130] I. V. Hertel and C.-P. Schulz. Atoms, Molecules and Optical Physics 2. Springer-Verlag Berlin Heidelberg, 1 edition (2015). ISBN 978-3-642-543135. doi:10.1007/978-3-642-54313-5. URL https://www. springer . com/la/book/ 9783642543128. 
[131] A. Gelessus, W. Thiel and W. Weber. Multipoles and Symmetry. Journal of Chemical Education (1995) 72(6) 505. doi:10.1021/ed072p505. URL http: //pubs.acs.org/doi/abs/10.1021/ed072p505.

[132] V. Roddatis, M. D. Bongers, R. Vink, V. Burlaka, J. Čížek and A. Pundt. Insights into Hydrogen Gas Environment-Promoted Nanostructural Changes in Stressed and Relaxed Palladium by Environmental Transmission Electron Microscopy and Variable-Energy Positron Annihilation Spectroscopy. The Journal of Physical Chemistry Letters (2018) 9(18) 5246-5253. doi:10.1021/acs.jpclett. 8b02363. URL https://pubs.acs.org/doi/10.1021/acs.jpclett.8b02363.

[133] T. Suzuki and R. Souda. The encapsulation of Pd by the supporting $\mathrm{TiO}_{2}(110)$ surface induced by strong metal-support interactions. Surface Science (2000) 448(1) 33-39. doi:10.1016/S0039-6028(99)01201-7. URL http: //www.sciencedirect.com/science/article/pii/S0039602899012017.

[134] R. E. Smallman and R. J. Bishop. Modern Physical Metallurgy and Materials Engineering. Elsevier, sixth edition (1999). ISBN 978-0-7506-4564-5. URL https://www.sciencedirect.com/science/book/9780750645645.

[135] M. Heidelmann, J. Barthel and L. Houben. StripeSTEM, a technique for the isochronous acquisition of high angle annular dark-field images and monolayer resolved electron energy loss spectra. Ultramicroscopy (2009) 109(12) 14471452. doi:10.1016/j.ultramic.2009.07.007. URL http://www.sciencedirect. com/science/article/pii/S030439910900182X.

[136] K. Sader, B. Schaffer, G. Vaughan, R. Brydson, A. Brown and A. Bleloch. Smart acquisition EELS. Ultramicroscopy (2010) 110(8) 998-1003. doi:10. 1016/j.ultramic.2010.01.012. URL http://www.sciencedirect.com/science/ article/pii/S0304399110000239.

[137] R. J. W. Kershaw. Hydrogen Content of the Air. Nature (1966) 209 190-191. doi:10.1038/209190a0. URL https://www. nature.com/articles/209190a0.

[138] Wikipedia, the free encyclopedia. Atomorbital (December 2017). URL https: //de.wikipedia.org/wiki/Datei:Orbitalesd.JPG.

[139] CreativeCommons. BY-SA 4.0 (December 2017). URL https:// creativecommons.org/licenses/by-sa/4.0/.

[140] The interactive Ellingham diagram (November 2017). URL https:// www.doitpoms.ac.uk/tlplib/ellingham_diagrams/interactive.php. Entry: palladium oxide. 
[141] S. Wagner, T. Kramer, H. Uchida, P. Dobron, J. Cizek and A. Pundt. Mechanical stress and stress release channels in 10-350 $\mathrm{nm}$ palladium hydrogen thin films with different micro-structures. Acta Materialia (2016) 114 116-125. doi:10. 1016/j.actamat.2016.05.023. URL http://www. sciencedirect.com/science/ article/pii/S1359645416303615. 


\section{Acknowledgment}

I would like to acknowledge and thank all the people that were involved in this work and contributed to its success.

First of all Astid Pundt for the outstanding supervision of this work. Her and the whole hydrogen in metals group for the valuable discussions and collaboration. Michael Seibt and Carsten Nowak for the additional supervision of this work. The members of the examination board: namely Astrid Pundt, Michael Seibt, Hans Hofsäss, Reiner Kirchheim, Vasily Moshnyaga and Martin Wenderoth. Carsten Nowak, Mike Kanbach, Karin Ahlborn, Johannes Maier, Björn Pfeiffer and Jonas Arlt for their help and support, especially with atom probe tomography. Mohsen Sotoudeh, Thorsten Stolper and Peter Blöchl for the illuminating discussions and magnificent collaboration for DFT calculations on rutile titanium dioxide. Martin Wenderoth for fruitful discussions in the context of semiconductor and metal/semiconductor interfaces. Niklas Herwig, Jorge de Heuvel and Maximilian Litschauer for their great work and high motivation in their bachelor thesis and the intensive collaboration that was associated with it. Vladimir Roddatis for his support, feedback and collaboration for TEM sample preparation and experiments on the ETEM, as well as for the great collaboration for publications. Matthias Hahn for his support on the different TEMs. Tobias Schulz for his amazing technical support. Michael Malchow for his always quick IT support. Christian Jooß and Jörg Hoffmann for letting me use their magentron sputtering system. Daniel Harding for the conduction of the ion-imaging measurements and for the borrowing of a $\operatorname{Pd}(111)$ single crystal. Michael Seibt and Christian Jooß

for their feedback to TEM and EELS related topics. Jakub Čížek from the Charles University (Prague) for the great collaboration in positron annihilation spectroscopy and accompanied discussions. Philipp Klose for his kind support with pole figure measurements. Julius Scholz for performing XPS measurements on the rutile substrate surfaces. The CRC1073 for offering and supporting trainings and further education. The to the CRC and IMP associated administration for their support in bureaucratic and financial matters. The people from the electronic and precision machine workshop for their support and help. All people that I might have forgotten to mention but were also involved in my work on the IMP.

It was a pleasure learning and studying at the institute of material physics and I personally wish all the best to everyone of the institute for the future.

Finally, I would like to thank my family, especially my parents and grandparents, for supporting me in my period of study (and before) and my girlfriend Julia Loth. In addition, I want to express my great gratitude to May L. Martin and Linda Röhrs 
who corrected this thesis without hesitation.

This work was supported by the DFG via SFB1073-C06 and DFG-PU131/9-2. 